\title{
Measurement of the Double Differential Inclusive Muon Neutrino Charged-current Cross-section in the NOvA Near Detector
}

Biswaranjan Behera

\author{
A Thesis Submitted to \\ Indian Institute of Technology Hyderabad \\ In Partial Fulfillment of the Requirements for \\ The Degree of Doctor of Philosophy
}

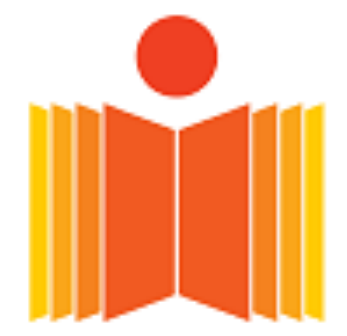

भारीीय प्रौयोगिबी चंस्थान ऐैस्राबाद

Indian institute of Tectnslogy Hyderibad

Department of Physics 


\section{Declaration}

I declare that this written submission represents my ideas in my own words, and where ideas or words of others have been included, I have adequately cited and referenced the original sources. I also declare that I have adhered to all principles of academic honesty and integrity and have not misrepresented or fabricated or falsified any idea/data/fact/source in my submission. I understand that any violation of the above will be a cause for disciplinary action by the Institute and can also evoke penal action from the sources that have thus not been properly cited, or from whom proper permission has not been taken when needed.

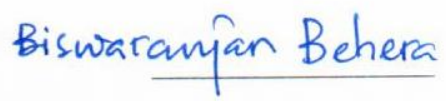

(Signature)

(Biswaranjan Behera)

PH13P1002

(Roll No.) 


\section{Approval Sheet}

This thesis entitled Measurement of the Double Differential Inclusive Muon Neutrino Charged-current Cross-section in the NOvA Near Detector by Biswaranjan Behera is approved for the degree of Doctor of Philosophy from IIT Hyderabad.

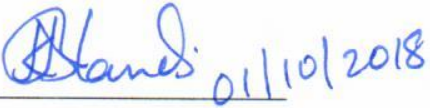

(Prof. B. K. Nandi) Examiner 1

Dept. of Physics, IITB

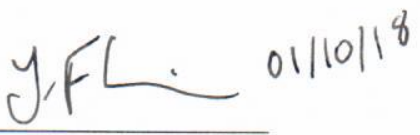

(Prof. James Libby) Examiner 2

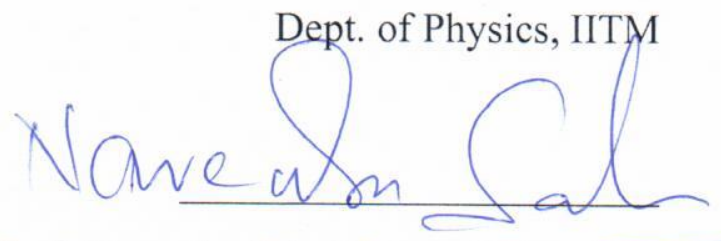

(Prof. Narendra Sahu) Internal Examiner

Dept. of Physics, IITH

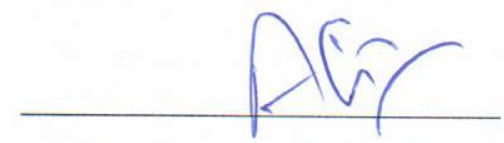

(Prof. Anjan K. Giri) Adviser

Dept. of Physics, IITH

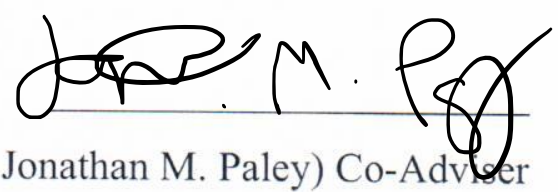

(Dr. Jonathan M. Paley) Co-AdvUer

Neutrino Divison, Fermilab

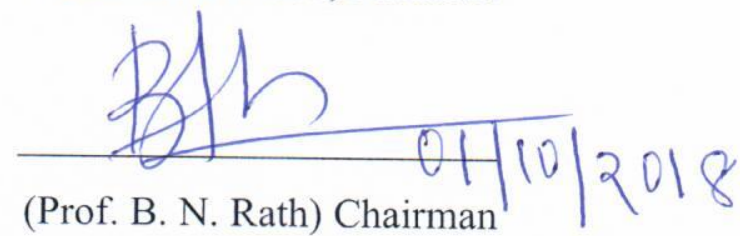

Dept. of Liberal Arts, IITH 


\section{Acknowledgements}

I thank lord Jagannath for his abundant blessings in my entire life endeavor. I owe my deepest gratitude to my supervisors Prof. Anjan K. Giri for his different kind of supports that to in different situations that give me to step forward to achieve my goal and the way he use to direct me always will be priceless moment for me.

Especially being special and unique Dr. Jonathan M. Paley for his constant support, guidance and motivation throughout my research work would be perpetual for me. In addition to this I am very much thankful to him for his precious time and scientific suggestions.

It was a privilege to work under their guidance and I am very proud of being their student.

I would like to thank also my mentors Dr. John Cooper, Dr. Peter Shanahan, Dr. Kanika Sachdev for giving their valuable time for scientific comments and suggestions for my improvement.

Besides my supervisors, I would like to thank the rest of my thesis committee members: Dr. Narendra Sahu and Dr. Vandana Sharma not only for reviewing my yearly progress and for giving me their insightful comments and encouragement but also for the motivations which incited me to widen my research from various perspectives. I would also like to thank all former and current Head of Department of Physics, teaching and non-teaching staff at IITH.

I am very much grateful to Dr. Fernanda Psihas, Dr. Gavin Davies and Jose Sepulveda for their immense help without any hesitation whenever I need irrespective of time.

I would also like to thank Koushik, Dr. Bibhu, Dr. Sujit, Dr. Asim for teaching me up to late night in coding as well as sharing food. I would like to extend my thanks to our Fermilab cricket team having fun with Navneet, Pavan, Prabhjot, Nitin, Reddy, Sijit, Rijeesh, Siva, Dr. Bibhuti.

My deep appreciation goes to Matt Judah, Ivan Lepetic and Jyoti for providing me a friendly and cheerful environment in the Fermilab to whom I share my office space. The thesis would not have come to a successful completion, without their help and co-operation. I am highly indebted to all my NOvA collaborators, especially Prof. Mark Messier, Prof. Matt Muether, Dr. Andrew Norman, Dr. G. Pawloski, Dr. Pengfei Ding, Dr. Evan Niner, Dr. Louise Suter, Dr. Michael Baird, Dr. Dan Pershey, Dr. Ashley Back, Dr. C. Backhouse, Dr. Keith Matera, Dr. J. Wolcott, Prof. Ryan Patterson, Erika Catano-Mur, Dr. Shih-kai Lin, Dr. B. Rebel, Dr. Leo Aliaga, Dr. A. Himmel, Dr. Ting Miao, Dr. R. Plunkett, Daisy, Justin, Micah, Teresa, Barnali, Ryan Murphy, Nitish. Without their precious support it would not have been possible to conduct this research.

I acknowledge support from DST, Govt of India and Fermilab, USA. I further acknowledge Fermilab administration for giving an access to the Fermilab laboratory and research facilities.

I am greatly thankful to my M.Sc. batch mates, especially Nirakar and Bikash for their financial and mental support whenever I need. My special thanks goes to Jogesh, Pradeep and Suchi, to whom I dump my frustration and also who helped me to get rid of my loneliness. I am also very thankful to my juniors Himanshu, Itishree, Ruby, Subha, Seema, Manas, Chita, Bijoy for providing me support during my research work.

I would like to extend huge and warm thanks to Kuldeep didi for her perceptive suggestions, constant motivation and support throughout my research work, shaping my personality as well healthy living atmosphere at Fermilab. In addition to this I can't stop without mentioning Suchi's contribution on reading and suggesting valuable comments on my thesis.

Last but not least I would like to take a long longbow especially to my parents for their unbounded, unconditional love, their understanding and the way they supported specially in adverse 
conditions where I can't stay without including to my grandfather's contributions in my nonacademic carrier. In addition to it if someone asks me about my younger brother Santosh Kumar then my tongue will start to say that without him my life is incomplete. The power he has is the management in all formats that he makes no pressure on others especially to my family members. And finally a special thanks to my sisters, uncle, aunt, aja and aii for their love, care and some important contributions into my life. Once again I salute and owe everything to them.

At the end, it is a pleasant task to express my thanks to all the people who made this thesis possible and an unforgettable experience for me. 


\section{Dedication}

To my Parents, Grandfather and Santosh Kumar. 


\begin{abstract}
NOvA (NuMI Off-axis $\nu_{e}$ Appearance) is the US flagship long-baseline neutrino oscillation experiments, beams of (anti)muon-neutrinos are used to precisely measure the rate of (anti)muon-neutrino disappearance and (anti)electron-neutrino appearance. The NOvA far detector (FD) is $810 \mathrm{~km}$ from the NuMI production target and positioned $14 \mathrm{mrad}$ off-axis from the NuMI beam, resulting in a narrow-band neutrino flux peaked around $2 \mathrm{GeV}$. The NOvA near detector (ND) is located approximately $1 \mathrm{~km}$ from the NuMI production target, off-axis such that the peak of the neutrino flux matches that of the far detector. However, the proximity of the ND to the production target means that it is exposed to a broader neutrino energy flux, and has a longer tail of neutrino interactions at higher energies than the FD. The high rate of interactions in the ND provides an opportunity for a rich program of neutrino-nucleus cross-section measurements.

This thesis showcases the measurement of the $\nu_{\mu}$ inclusive charged current cross-section using the NOvA Near Detector (ND) data. The neutrino beam in the ND peaks near $2 \mathrm{GeV}$ and allows a unique probe of the turn on and turn off of different interaction modes available to neutrinos. We present a flux-averaged double-differential inclusive cross-section measurement of muon-neutrino charged-current interactions in the NOvA near detector as a function of the muon energy and angle. We also present the measurement of the cross-section as a function of neutrino energy in a restricted phase space of the muon kinematics. The data used for this measurement consist of $8.09 \times 10^{20} \mathrm{POT}$ of neutrino beam collected between August 2014 and February 2017.
\end{abstract}




\section{Contents}

Declaration ............................... ii

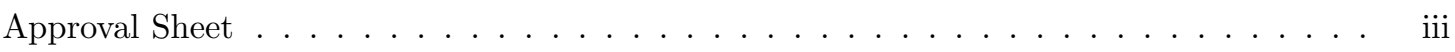

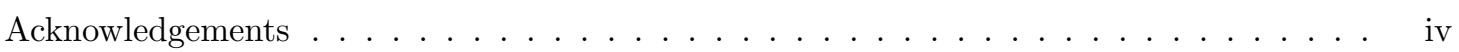

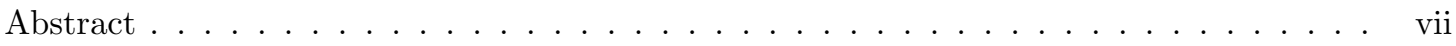

Nomenclature $\quad x$

1 Introduction $\quad 1$

1.1 Standard Model of Particle Physics . . . . . . . . . . . . . . . . . . . . . . 1

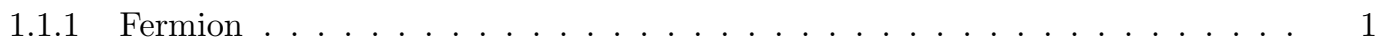

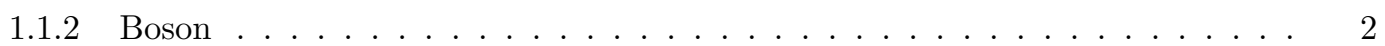

1.2 Beyond the standard model . . . . . . . . . . . . . . . . . . 2

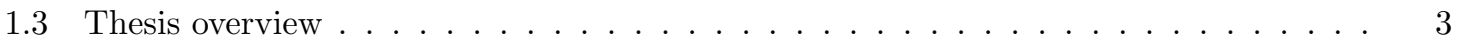

2 Neutrino Physics 5

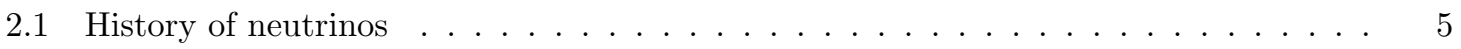

2.2 Neutrino Oscillation . . . . . . . . . . . . . . . . . . . . 6

2.2 .1 Neutrino Oscillations in Vacuum . . . . . . . . . . . . . . . 7

2.2 .2 Neutrino Oscillations in Matter . . . . . . . . . . . . . . . 8

2.3 Importance of Cross-section . . . . . . . . . . . . . . . . . . . . . . . . . 10

2.4 Neutrino Kinematics . . . . . . . . . . . . . . . . . . . . . . . . . . 11

2.5 Neutrino Nucleus Interaction . . . . . . . . . . . . . . . . . . 12

2.5.1 Quasi-elastic Interaction $(\mathrm{QE}) \quad \ldots \ldots \ldots \ldots \ldots$

2.5.2 Resonance Pion Production (Res) _ . . . . . . . . . . . . . . 15

2.5.3 Coherent Scattering $(\mathrm{Coh}) \ldots \ldots \ldots \ldots$

2.5.4 Deep-inelastic Scattering (DIS) _ . . . . . . . . . . . . . . 17

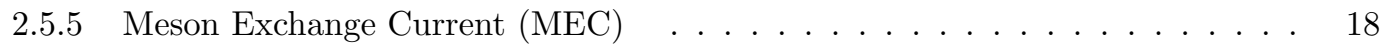

2.6 Nuclear Effects . . . . . . . . . . . . . . . . . . . . . . . . . . . . . . . 19

2.6.1 Relativistic Fermi Gas Model (RFG) . . . . . . . . . . . . . . . . . . 19

2.6.2 Short Range Correlation $(\mathrm{SRC}) \ldots \ldots \ldots . \ldots \ldots$

2.6.3 Random Phase Approximation (RPA) . . . . . . . . . . . . . . . . 21

2.6 .4 Final State Interactions $(\mathrm{FSI}) \ldots \ldots \ldots \ldots \ldots$ 
3 The NOvA Experiment 23

3.1 NuMI Neutrino Beam . . . . . . . . . . . . . . . . . . . . . . . . 23

3.1.1 Fermilab Accelerator Complex . . . . . . . . . . . . . . 23

3.1 .2 NuMI Beamline . . . . . . . . . . . . . . . . . . . . 24

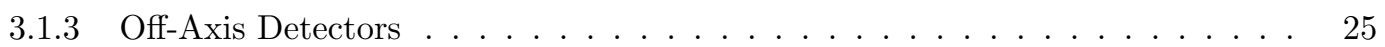

3.2 The NOvA Detectors . . . . . . . . . . . . . . . . . . . . . . . 27

3.2 .1 The Cell . . . . . . . . . . . . . . . . . . . 27

3.2.2 The Liquid Scintillator . . . . . . . . . . . . . . . . . . . 29

3.2 .3 The APD . . . . . . . . . . . . . . . . . . . . . . . 29

3.2.4 Far Detector . . . . . . . . . . . . . . . . . 30

3.2 .5 Near Detector . . . . . . . . . . . . . . . . . . . . 30

3.3 Overview of Data Acquisition System . . . . . . . . . . . . . . . . . . . . . 32

3.4 Performance of the NOvA Detectors . . . . . . . . . . . . . . . . . . . . 33

3.5 Exposure .............................. 33

4 Simulation $\quad 35$

4.1 Flux Prediction . . . . . . . . . . . . . . . . . . . . . . . . 35

4.2 Neutrino Interactions . . . . . . . . . . . . . . . . . . . . . . . . . . . 35

4.3 Propagation of Particles . . . . . . . . . . . . . . . . . . . . . 36

4.4 Transport of Photon in Detector . . . . . . . . . . . . . . . . . . . . . . 37

4.5 Readout Simulation . . . . . . . . . . . . . . . . . . . . . . 37

4.6 The APD Sag Simulation . . . . . . . . . . . . . . . . . . . . 39

4.7 Cherenkov Light Simulation . . . . . . . . . . . . . . . . . . . . . . . . . . . . 39

4.8 Birks-Chou Parameters ......................... . . . 40

5 Reconstruction and Calibration $\quad 41$

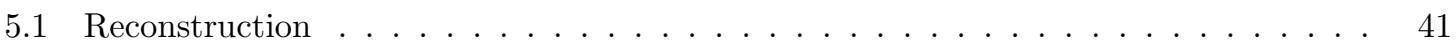

5.1 .1 Isolating Neutrino Interactions . . . . . . . . . . . . . . . . . 41

5.1 .2 Identifying Lines with Multi-Hough Transform . . . . . . . . . . . . . . . . 43

5.1.3 Identification of Vertex using Elastic Arms . . . . . . . . . . . . . . . . 44

5.1.4 Prong Formation with Fuzzy k-Means . . . . . . . . . . . . . . . . . 45

5.1 .5 Kalman Track . . . . . . . . . . . . . . . . . . . . 46

5.2 Calibration ............................ . . . 47

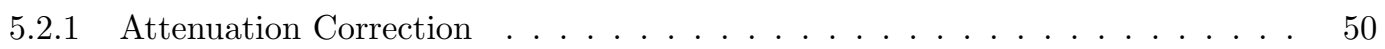

5.2 .2 Absolute Energy Scale . . . . . . . . . . . . . . . . . . 52

6 Measurement of Inclusive $\nu_{\mu}$ CC Cross-section $\quad 53$

6.1 Analysis Overview .......................... . 53

6.1.1 Simulation, Reconstruction and Data set details . . . . . . . . . . 55

6.2 Systematic Uncertainties . . . . . . . . . . . . . . . . . . . . . 55

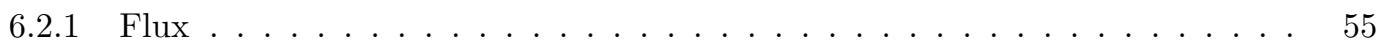

6.2.2 Neutrino-nucleus interaction modeling . . . . . . . . . . . . . 56

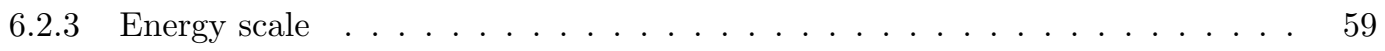

6.2.4 Light Model . . . . . . . . . . . . . . . . . . . . 59 
6.3 Event selection . . . . . . . . . . . . . . . . . . . . . . 60

6.3.1 Event Quality and Spill Cuts . . . . . . . . . . . . . . . 60

6.3.2 Initial Fiducial and Containment Cut . . . . . . . . . . . . . . . . . 61

6.3 .3 Muon Identification . . . . . . . . . . . . . . . . . . . 61

6.3.4 MuonID Cut Optimization .................. 62

6.3.5 Optimization of Fiducial Volume and Containment Criteria . . . . . . . . . . 66

6.3.6 Event Selection Cut Flow Tables . . . . . . . . . . . . . . . . . 68

6.4 Muon and Neutrino Energy Estimation, Resolution and Binning . . . . . . . . . . . 69

6.4 Muon Energy . . . . . . . . . . . . . . . . . . . . . . 69

6.4.2 Hadronic Energy . . . . . . . . . . . . . . . . . . . . . 70

6.4.3 Optimization of Polynomial Fitting . . . . . . . . . . . . . 70

6.4.4 Resolution and Binning ................... 70

6.4.5 Muon Kinematic Cuts . . . . . . . . . . . . . . . . . . . . 73

6.5 Efficiency Correction . . . . . . . . . . . . . . . . . . . . . . . 74

6.5.1 Multidimensional Approach to Efficiency Correction . . . . . . . . . . . . 82

6.6 Unfolding . . . . . . . . . . . . . . . . . . . . . . . . . 82

6.7 Flux Calculation ........................... . 87

6.8 Target Counting . . . . . . . . . . . . . . . . . . . . . . 91

6.9 Additional Systematics . . . . . . . . . . . . . . . . . . . . . . . . . 92

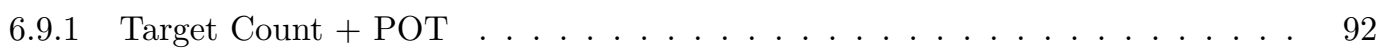

6.9 .2 Intensity Effects . . . . . . . . . . . . . . . . . . . 92

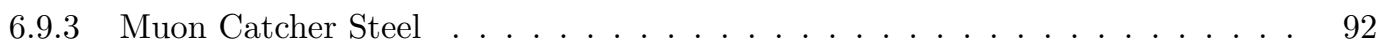

6.9 .4 Alignment . . . . . . . . . . . . . . . . . . 93

6.9.5 Particle Propagation Model .................. 93

$\begin{array}{llr}7 & \text { Results and Conclusion } & 95\end{array}$

$\begin{array}{lr}\text { References } & 100\end{array}$ 


\section{Chapter 1}

\section{Introduction}

\subsection{Standard Model of Particle Physics}

Out of all possible ideas proposed to understand the universe, the standard model (SM) is the most established theoretical framework which describes the interaction between the elementary particles at the most fundamental level and could accommodate almost all the observed experimental data. This model encounters two types of particles: (i) fermions, (ii) bosons.

\subsubsection{Fermion}

Fermions are odd half-integral spin subatomic particles which make up the matter and obey FermiDirac statistics. These are further categorized into quarks and leptons, which are the elementary particles with no further substructure and composed of all the visible matters. Each group of leptons and quarks are classified into three generations known as, first, second and third generations.

\section{Quark}

There exists six flavors of quarks namely, up (u), down (d), charm (c), strange (s), top (t), bottom (b). Quarks of different flavors combine with each other to form hadrons, which in general are of two types (a) Baryons, which have a triquark state and (b) Mesons, which are made up of a quark and an anti-quark. Baryons are associated with baryon quantum numbers, which should be conserved in the SM. The problem arising in triquark state associated with the Pauli-exclusion principle gave birth to a new quantum number called "color charge, which is of three types, namely red (R), green $(\mathrm{G})$ and blue (B). The corresponding anti-quarks have anti-colors as the composite particles should be colorless.

\section{Lepton}

As like quark, lepton also has six flavors which consists of charged leptons namely electron (e), muon $(\mu)$, tau $(\tau)$ and the corresponding neutral leptons known as electron neutrino $\left(\nu_{e}\right)$, muon neutrino $\left(\nu_{\mu}\right)$, tau neutrino $\left(\nu_{\tau}\right)$. Leptons are color singlet and do not carry any color quantum number. Each family of leptons is linked with the corresponding lepton number $L_{i}$. 


\subsubsection{Boson}

Bosons are elementary integral spin particles which transmit forces and satisfy the Bose-Einstein statistics. These are of two types (a) vector gauge bosons with spin one (gluon $(g)$, photon $(\gamma)$, $W^{ \pm}, Z^{0}$ ), and (b) scalar Higgs boson with spin zero. Gluon is a bi-color massless vector boson which mediates strong interaction. Quantum chromodynamics (QCD) is the theory of this color force with coupling strength represented by the QCD coupling constant $\left(\alpha_{s}\right)$. This interaction is responsible for the strong binding of quarks and leptons inside the hardons. The strong force, as well as the electromagnetic, weak and gravitational forces, are the four elemental forces of our nature. Electromagnetic interactions can be well explained by quantum electrodynamics (QED) and the corresponding coupling strength is represented in terms of the fine structure constant $\left(\alpha_{\mathrm{em}}\right)$. The colorless and massless photon is the carrier of this interaction. The mediator for weak force is $W^{ \pm}$ and $Z^{0}$ bosons. This interaction permits quarks to exchange their flavors with each other. The strength of this force is represented by Fermi constant $\left(G_{F}\right)$. Gravitational force is the weakest fundamental force, which is mediated by Graviton. Graviton is an exceptional hypothetical gauge boson with spin 2 , which don't have any experimental proof so far regarding its extent. The summary of the fundamental interactions is presented in Table 1.1.

\begin{tabular}{|c|c|c|c|c|}
\hline Forces & Gauge group & Mediator & Range $(\mathrm{m})$ & Coupling strength \\
\hline \hline Strong & $S U(3)_{C}$ & gluon & $\leq 10^{-15}$ & $<1$ \\
Weak & $S U(2)_{L}$ & $W^{ \pm}, Z^{0}$ & $10^{-18}$ & $10^{-6}$ \\
Electromagnetic & $U(1)_{Y}$ & photon & infinity & $1 / 137$ \\
Gravitational & $\cdots$ & graviton & infinity & $10^{-39}$ \\
\hline
\end{tabular}

Table 1.1: Summary of fundamental interactions in the SM.

Glashow, Salam, and Weinberg unify the electromagnetic and weak forces $[1,2,3]$ into the electroweak interaction, which again combines with strong interaction to form a single unifying model, named as the standard model. The SM is represented by the $S U(3)_{C} \times S U(2)_{L} \times U(1)_{Y}$ gauge group, where $S U(3)_{C}$ is for strong interaction where $C$ stands for the color charge, $S U(2)_{L}$ is for weak interaction, where $L$ implies the coupling of $W^{ \pm}, Z^{0}$ bosons with only left-handed fermions and $U(1)_{Y}$ stands for electromagnetic force with $Y=2\left(Q-T_{3}\right)$ as the hypercharge. The masses of the fermions and bosons are measured experimentally. However, the SM gauge symmetry imposes the SM particles to be massless. Then, how $W^{ \pm}, Z^{0}$ bosons get mass, i.e. are massive? The answer to this puzzle is Higgs mechanism [4, 5, 6, 7], where a spontaneous symmetry breaking of $S U(2)_{L} \times U(1)_{Y} \rightarrow U(1)_{\mathrm{em}}$ occurs. Due to this symmetry breaking, the vacuum expectation value (VEV) of the new hypothetical scalar field is found to be zero, which will give masses to the weak gauge bosons. The interactions of this hypothetical scalar field with fermions provide masses to them. This hypothetical particle is called Higgs boson, which was discovered in 4th July 2012 by the LHC Collaboration $[8,9]$. In Table 1.2, we represent the properties of SM particle contents under the $S U(3)_{C} \times S U(2)_{L} \times U(1)_{Y}$ gauge group.

\subsection{Beyond the standard model}

Though the SM of elementary particle physics is a profound and a proficient theory, it fails to explain many physical phenomena like matter-antimatter asymmetry, neutrino mass, gravitational force and 


\begin{tabular}{|c|c|c|}
\hline & Fields & $\left(S U(3)_{C}, S U(2)_{L}, U(1)_{Y}\right)$ \\
\hline \hline \multirow{2}{*}{ Quarks } & $\left(\begin{array}{l}u \\
d\end{array}\right)_{L},\left(\begin{array}{c}c \\
s\end{array}\right)_{L},\left(\begin{array}{l}t \\
b\end{array}\right)_{L}$ & $(3,2,1 / 6)$ \\
& $u_{R}, c_{R}, t_{R}$ & $(3,1,2 / 3)$ \\
& $d_{R}, s_{R}, b_{R}$ & $(3,1,-1 / 3)$ \\
\hline Leptons & $L_{L}=\left(\begin{array}{c}e \\
\nu_{e}\end{array}\right)_{L},\left(\begin{array}{c}\mu \\
\nu_{\mu}\end{array}\right)_{L},\left(\begin{array}{c}\tau \\
\nu_{\tau}\end{array}\right)_{L}$ & $(1,2,-1 / 2)$ \\
& $H$ & $(1,1,-1)$ \\
\hline Higgs & $H, \tau_{R}$ & $(1,2,1)$ \\
\hline
\end{tabular}

Table 1.2: Quantum numbers of SM particles under $S U(3)_{C} \times S U(2)_{L} \times U(1)_{Y}$ gauge group.

it does not have any component of dark matter and dark energy etc. These failures of SM implies that there should be new physics beyond the SM. The most general demerits of SM include the following unresolved puzzles.

- The elementary particles are organized into three families. But there is no proper reason for the existence of only 3 generations. So, one interesting question arises here, "Why there is only three family of leptons and quarks".

- We do not have any idea, why the masses of third generation particles are more massive than the second generation which has comparatively higher mass than the first generation fundamental particles of the universe. Thus, the next puzzle of the SM is, "why there is mass hierarchy".

- Though the equal amount of particle and antiparticle was produced during the time of big-bang, the existence of star, galaxy and our universe implies the dominance of matter over antimatter. Here the most existing question arise, "why the present universe is matter dominated".

- SM does not have any explanation for the gravitational force, which is the fourth fundamental interaction of nature.

- The components of dark matter and dark energy are absent in the SM, though $95 \%$ our universe belong to the invisible sector.

- Though neutrinos are massless in the SM, the confirmation of oscillation between different flavors of neutrino imply that they have mass.

These open questions in the SM forced physicists to think beyond it. Now, theoreticians are building new physics models in order to resolve these problems. Besides these open puzzles, there are many issues in the neutrino sector, which we are going to explain in the next chapter.

\subsection{Thesis overview}

In this chapter, we discuss the particle content of the SM. We also briefly wrote the success and failure of the SM, which indicates the presence of new physics beyond it. Chapter 2 explains mostly the theory behind neutrino physics and neutrino-nucleus interaction. Next, to it, an overview of the NOvA experiment is briefly discussed in Chapter 3. Chapter 4 summaries the simulation chain 
used in the NOvA experiment followed by reconstruction and calibration in Chapter 5. The detail discussion about the analysis and its results is described in Chapter 6 . 


\section{Chapter 2}

\section{Neutrino Physics}

Neutrinos are one of the most prolific subatomic particles of the standard model. Neutrinos were taken birth very soon after the Big Bang. These are constantly being produced from the explosions of supernovae, deaths of stars and nuclear reactions on earth. We have no idea regarding the passes of around hundred trillions of neutrinos across our body in every second. These are the lightest colorless fermions, which do not carry electric charge, thus couple very rarely with other SM fermions by weak interaction only. This feeble interaction of neutrinos makes them the least understood fundamental particles. Everyone was unknown about this particle until the beta decay puzzles came to picture.

\section{$2.1 \quad$ History of neutrinos}

The area of neutrino physics started with the proposal of the existence of new hypothetical neutral fermion by the theorist Wolfgang Pauli in 1930 [10]. Before the prediction on neutrino, beta decay was considered to be a two-body decay process,

$$
n^{0} \rightarrow p^{+}+e^{-} .
$$

Since the daughter nuclei are almost at rest, one should expect discrete energy spectrum for electron particle due to the energy conservation. However, the distribution of electron energy values is found to be continuous [11]. Furthermore, the direction of recoiling nuclide was not completely opposite to the direction of the emitted electron. Thus, the energy-momentum is not conserved in this process. Since neutrino with spin half decays into two spin half fermions in the $\beta$-decay, it also violates the conservation of angular momentum. In order to explain the lack of conservation energy, momentum, and angular momentum, Pauli suggested the emission of a new neutral spin half fermion with no mass, named as Neutron along with the electron. After the discovery of the neutron by J. Chadwick in 1932 [12], Fermi renamed the neutral particle suggested by Pauli as "Neutrino" (Italian: "little neutral one") in 1934 and has developed the theory of beta decay, known as Fermi theory [13, 14].

Since neutrinos have very weak interaction with the ordinary matters, they are very difficult to detect in a detector. In 1956, Fred Reines and Clyde Cowan were planned to search for antineutrino in the inverse $\beta$-decay process $[15,16,17,18]$. In this process, the anti particle of neutrinos produced 
in the nuclear reactor united with protons, creating neutron and positron.

$$
\overline{\nu_{e}}+p^{+} \rightarrow n^{0}+e^{+} .
$$

Afterward, the pair annihilation process occurs, i.e., the positron combines with an electron to produce two gamma rays,

$$
e^{+}+e^{-} \rightarrow \gamma+\gamma
$$

and the neutron is captured by the cadmium atoms producing a photon

$$
n+C d^{108} \rightarrow C d^{109}+\gamma
$$

This was the first experiment, where the anti-electron-neutrino $\left(\nu_{e}\right)$ has detected, for which Reines and Cowan won the Nobel Prize in 1995.

After the observation of $K^{0}-\bar{K}^{0}$ mixing in the quark sector, Pontecorvo has proposed the possibility of neutrino mixing in 1957 [19]. Since only electron neutrino was known at that time, he changed his idea to the existence of neutrino-antineutrino oscillation. In 1962, a new type of neutrino was discovered at Brookhaven National Laboratory (BNL) [20], which show different behaviors with electron and muon. This was the discovery of the second neutrino, known as muon neutrino $\left(\nu_{\mu}\right)$ for which Lederman, Schwartz, and Steinberger have received Nobel Prize in 1988. Just after the discovery of muon neutrino, Maki, and Sakata have developed the theory of $\nu_{e} \leftrightarrows \nu_{\mu}$ oscillation in 1962 [21]. Thereafter, the DONUT experiment has discovered the tau neutrino 2000 [22]. In 2015, Takaaki Kajita and Arthur B. McDonald together have awarded Nobel Prize for their contribution in the discovery of neutrino oscillations, which shows that neutrinos have mass.

\subsection{Neutrino Oscillation}

Neutrino comes with three flavors and changes flavor as they travel from one place to another irrespective of medium means through earth or vacuum. The amount they change depends on how much time they have to change. This time is directly related to the distance of the neutrino traveled and the energy of the neutrino itself. Distance is much easier to measure than the neutrino energy estimation. Neutrino oscillations occur because neutrino flavor states are a quantum superposition of mass eigenstates.

$$
\left|\nu_{\alpha}\right\rangle=\sum_{i} U_{\alpha i}\left|\nu_{i}\right\rangle
$$

where, $\left|\nu_{\alpha}\right\rangle$ is the flavor eigenstate, where $\alpha=\mathrm{e}, \mu, \tau$ and $\left|\nu_{i}\right\rangle$ is the mass eigenstate and $\mathrm{i}=1,2,3$. In quantum mechanics, the neutrino can be described either in flavor bases or mass eigenbases. They are related with a unitary leptonic mixing matrix $U_{\alpha i}$, commonly known as Pontecorvo-MakiNakagawa-Sakata (PMNS) matrix. In general, it can be written as follows:

$$
\left(\begin{array}{l}
\nu_{e} \\
\nu_{\mu} \\
\nu_{\tau}
\end{array}\right)=U\left(\begin{array}{l}
\nu_{1} \\
\nu_{2} \\
\nu_{3}
\end{array}\right),
$$




\subsubsection{Neutrino Oscillations in Vacuum}

Let us consider a given neutrino source at position $\mathrm{x}=0$, and time $\mathrm{t}=0$. After certain distance of travel by neutrino let us say position and time are $\mathrm{x}$ and $\mathrm{t}$ respectively. One can obtain the evolution of neutrino mass state in time using the Schrodinger equation. The solution of the equation provides a plane wave equation associated with a phase.

The mass eigen state of neutrino at a point in space-time $(x, t)$ is given by

$$
\begin{aligned}
\left|\nu_{\alpha}(x, t)\right\rangle & =\sum_{i=1}^{n} U_{\alpha i}\left|\nu_{i}(x, t)\right\rangle \\
& =\sum_{i=1}^{n} U_{\alpha i} e^{-i \phi_{1}}\left|\nu_{i}(0,0)\right\rangle
\end{aligned}
$$

The transition amplitude $A$ can be written for $\alpha$ neutrino flavor at space-time $(0,0)$ to $\beta$ neutrino flavor at later space-time $(x, t)$ is:

$$
\begin{aligned}
A\left(\nu_{\alpha} \rightarrow \nu_{\beta}\right) & =\left\langle\nu_{\beta}(x, t) \mid \nu_{\alpha}(x, t)\right\rangle \\
& =\sum_{i=1}^{n} \sum_{j=1}^{n} U_{\beta j}^{*} U_{\alpha i}\left\langle\nu_{j}(x, t) \mid \nu_{i}(0,0)\right\rangle \\
& =\sum_{i=1}^{n} U_{\alpha i} U_{\beta i}^{*} e^{i \phi_{i}} .
\end{aligned}
$$

The transition probability is

$$
\begin{aligned}
P_{\alpha \beta} & =\left|A\left(\nu_{\alpha} \rightarrow \nu_{\beta}\right)\right|^{2} \\
& =\left|\sum_{i=1}^{n} U_{\alpha i} U_{\beta i}^{*} e^{i \phi_{i}}\right|^{2} \\
& =\sum_{i=1}^{n} \sum_{j=1}^{n} U_{\alpha i} U_{\beta i}^{*} U_{\alpha j}^{*} U_{\beta j} e^{-i\left(\phi_{j}-\phi_{i}\right)} .
\end{aligned}
$$

Considering neutrino a relativistic particle, the energy $E_{i}$ of neutrino mass eigenstates $\nu_{i}$, with mass $m_{i}$, can be approximated as

$$
E_{i}=\sqrt{p_{i}^{2}+m_{i}^{2}} \simeq p+\frac{m_{i}^{2}}{2 E}
$$

The phase difference tells us about the neutrino mass splitting.

$$
\phi_{i}-\phi_{j}=\left(E_{i}-E_{j}\right) t-\left(p_{i}-p_{j}\right) x=\frac{\Delta m_{i j}^{2} L}{2 E}
$$

where $\Delta m_{i j}^{2}=m_{i}^{2}-m_{j}^{2}$.

If neutrino comes with two flavors, they are related with a rotation matrix and $\theta$ is the mixing 
angle.

$$
U=\left(\begin{array}{cc}
\cos \theta & \sin \theta \\
-\sin \theta & \cos \theta
\end{array}\right)
$$

The oscillation probability of a neutrino $\nu_{\alpha}$ oscillates into $\nu_{\beta}$ for the two flavor neutrinos is

$$
\mathrm{P}_{\nu_{\alpha} \rightarrow \nu_{\beta}}\left(\mathrm{E}_{\nu}\right)=\sin ^{2} 2 \theta \sin ^{2}\left(\frac{1.27 \Delta m_{21}^{2} \mathrm{~L}}{4 \mathrm{E}_{\nu}}\right)
$$

The neutrino oscillation probability depends upon L/E ratio and sinusoidal function. From Eq. 2.13 it is evident that neutrino oscillations are actually caused by neutrino masses within the measured energy region. If the mass squared difference is zero, the oscillation probability is zero, implying the existence of at least one non-zero neutrino mass state. So far we have observed experimentally only mass squared difference, not the absolute mass of neutrino. Furthermore, $\Delta m^{2}$ is involved in sine square, which means that there is no sensitivity of the sign of $\Delta m^{2}$. Again the mixing angle is

involved in terms of $\sin ^{2} 2 \theta$, in two flavor framework, the oscillation amplitude is same for at mixing angle $40^{\circ}$ or $50^{\circ}$. But, they resulted differently in three neutrino oscillation framework.

Extending the two flavor neutrino oscillation to three flavor oscillation, the $3 \times 3$ unitary PMNS matrix $U$ can be expressed as

$$
U=\left(\begin{array}{ccc}
c_{13} c_{12} & c_{13} s_{12} & s_{13} e^{-i \delta} \\
-c_{23} s_{12}-s_{23} c_{12} s_{13} e^{i \delta} & c_{23} c_{12}-s_{23} s_{12} s_{13} e^{i \delta} & c_{13} s_{23} \\
s_{23} s_{12}-c_{23} c_{12} s_{13} e^{i \delta} & -s_{23} c_{12}-c_{23} s_{12} s_{13} e^{i \delta} & c_{13} s_{23}
\end{array}\right),
$$

where $\mathrm{c}_{i j}=\cos \theta_{i j}$ and $\mathrm{s}_{i j}=\sin \theta_{i j}$.

One more interesting feature is that charge-parity $(\mathrm{CP})$ violation comes in to picture in three flavor neutrino oscillation framework and it is not seen in two flavor case. The transition probability of a neutrino $\nu_{\alpha}$ oscillates into $\nu_{\beta}$ for a 3 -flavor neutrino can be expressed using $3 \times 3$ unitary PMNS matrix $U_{i j}$ as,

$$
\begin{aligned}
P_{\nu_{\alpha} \rightarrow \nu_{\beta}} & =\delta_{\alpha \beta}-2 \sum_{i>j} \Im\left[U_{\beta j}^{\star} U_{\alpha j} U_{\beta i} U_{\alpha i}^{\star}\right] \sin \frac{\Delta m_{j i}^{2} L}{2 E} \\
& +4 \sum_{i>j} \Re\left[U_{\beta j}^{\star} U_{\alpha j} U_{\beta i} U_{\alpha i}^{\star}\right] \sin ^{2} \frac{\Delta m_{j i}^{2} L}{4 E}
\end{aligned}
$$

\subsubsection{Neutrino Oscillations in Matter}

When electron neutrinos propagate through matter they interact with the electron content of the matter, because neutrinos interact with their corresponding leptons. The electron neutrinos interact with electrons in the matter under both charged and neutral current process, but for other flavors only neutral current interactions are possible. So it experiences a flavor asymmetric potential, so-called the Wolfenstein term, which is popularly known as the Mikheyev- Smirnov-Wolfenstein (MSW) effect [23].

For simplicity, starting with 2-flavor, MSW effect can be expressed according to the Schrodinger 
equation as:

$$
\begin{aligned}
i \frac{d}{d L}\left(\begin{array}{c}
\nu_{e} \\
\nu_{\mu}
\end{array}\right) & =\left(U\left(\begin{array}{cc}
\frac{m_{1}^{2}}{2 E} & 0 \\
0 & \frac{m_{2}^{2}}{2 E}
\end{array}\right) U^{*}+V_{c c}\left(\begin{array}{cc}
1 & 0 \\
0 & 0
\end{array}\right)\right)+\left(\begin{array}{c}
\nu_{e} \\
\nu_{\mu}
\end{array}\right) \\
& =\frac{1}{4 E}\left(\begin{array}{cc}
-\Delta m^{2} \cos 2 \theta+4 E V_{c c} & \Delta m^{2} \sin 2 \theta \\
\Delta m^{2} \sin 2 \theta & \Delta m^{2} \cos 2 \theta
\end{array}\right)\left(\begin{array}{c}
\nu_{e} \\
\nu_{\mu}
\end{array}\right)
\end{aligned}
$$

where $V_{c c}= \pm \sqrt{2} \mathrm{G}_{F} \rho_{e}, G_{F}$ is the Fermi constant, $\rho_{e}$ is the electron number density, and the positive and negative signs are for neutrinos and anti-neutrinos respectively.

The effective Hamiltonian in the mass basis can be derived from the diagonalization of effective Hamiltonian in the flavor basis through a unitary matrix.

$$
U_{M}^{T} \mathcal{H}_{F} U_{M}=\mathcal{H}_{M},
$$

where $\mathcal{H}_{F}$ is effective Hamiltonian flavor basis, $U_{M}$ is the effective mixing matrix in matter

$$
U_{M}=\left(\begin{array}{cc}
\cos \theta_{M} & \sin \theta_{M} \\
-\sin \theta_{M} & \cos \theta_{M}
\end{array}\right),
$$

and $\mathcal{H}_{M}$ is effective Hamiltonian in mass basis, expressed as

$$
\mathcal{H}_{M}=\frac{\Delta m^{2}}{4 E}\left(\begin{array}{cc}
-1 & 0 \\
0 & 1
\end{array}\right)
$$

Simplifying Eq. 2.19 and Eq. 2.18, the effective mass-squared differences and mixing angles can be expressed as

$$
\begin{array}{r}
\Delta m_{M}^{2}=\sqrt{\left(\Delta m^{2} \cos 2 \theta \mp 2 E V_{c c}\right)^{2}+\left(\Delta m^{2} \sin 2 \theta\right)^{2}} \\
\tan 2 \theta_{M}=\frac{\tan 2 \theta}{1 \mp \frac{2 E V_{c c}}{\Delta m^{2} \cos 2 \theta}},
\end{array}
$$

where $\Delta m^{2}$ and $\theta$ represent mass splitting and mixing angle in vacuum. The matter effect modifies the oscillation parameters based on the matter density and neutrino energy. When $\cos 2 \theta=2 E V_{c c}$ it is found that $\Delta m_{M}^{2}=\Delta m^{2}$, which leads to a resonance. This resonance corresponds to the electron number density as :

$$
\begin{aligned}
V_{c c} & =\sqrt{2} G_{F} \rho_{e} \\
& =\frac{\Delta m^{2} \cos 2 \theta}{2 E} \\
\rho_{\text {res }} & =\frac{\Delta m^{2} \cos 2 \theta}{2 \sqrt{2} G_{F} E} .
\end{aligned}
$$

At resonance, the effective mixing angle becomes maximal $\left(45^{\circ}\right)$ which leads to an amplification of oscillation probabilities. Matter effect will be significant if neutrino pass through more dense material, for example at the core of the Sun. This method is used to fix the mass ordering of neutrinos in solar neutrino mixing, and long-baseline neutrino oscillation experiments to find the mass ordering 
of $\nu_{3}$. Furthermore, as positive and negative sign denotes for neutrino and anti-neutrino respectively, the oscillation probability will be different, so there is an expected charge-parity asymmetry. The oscillation probability for muon neutrinos oscillating into electron neutrinos is

$$
\begin{aligned}
P_{\nu_{\mu} \rightarrow \nu_{e}} & =\sin ^{2} \theta_{23} \sin ^{2} 2 \theta_{13} \frac{\sin ^{2}\left(\Delta m_{31}^{2} \mp a L\right)}{\left(\Delta m_{31}^{2} \mp a L\right)^{2}} \Delta m_{31}^{4} \\
& +\sin 2 \theta_{23} \sin 2 \theta_{13} \sin 2 \theta_{12} \frac{\sin \left(\Delta m_{31}^{2} \mp a L\right)}{\left(\Delta m_{31}^{2} \mp a L\right)} \Delta m_{31}^{2} \frac{\sin (\mp a L)}{(\mp a L)} \Delta m_{21}^{2} \\
& \left(\cos \Delta m_{32}^{2} \cos \delta \mp \sin \Delta m_{32}^{2} \sin \delta\right) \\
& +\cos ^{2} \theta_{23} \sin ^{2} 2 \theta_{12} \frac{\sin ^{2}(\mp a L)}{(\mp a L)^{2}} \Delta m_{21}^{4},
\end{aligned}
$$

where $a=\frac{G_{F} \rho_{e}}{\sqrt{2}}$, and minus and plus signs are for neutrinos and anti-neutrinos respectively.

\subsection{Importance of Cross-section}

Neutrino oscillation experiments do not measure the oscillation probability, but rather the event rate. So we need to measure the number of neutrinos as a function of neutrino energy at near and far detectors. The number of neutrinos at the near detector is convolution of flux, cross-section and efficiency of near detector.

$$
\mathrm{N}_{\nu_{\alpha}}^{\mathrm{ND}}\left(\mathrm{E}_{\nu}\right) \propto \phi\left(\mathrm{E}_{\nu}\right) \times \sigma\left(\mathrm{E}_{\nu}\right) \times \epsilon^{\mathrm{ND}}\left(\mathrm{E}_{\nu}, \mathrm{T}_{\alpha}, \Theta_{\alpha} \cdots\right)
$$

Flux $\left(\phi\left(\mathrm{E}_{\nu}\right)\right)$ is defined as number of neutrinos produced by the accelerator per $\mathrm{cm}^{2}$, per energy, for a given number of protons on target. The probability of interaction of the neutrinos in the material of the detector is known as cross-section $\left(\sigma\left(\mathrm{E}_{\nu}\right)\right)$ and efficiency $(\epsilon)$ corrects for events which we lose during selection (threshold, acceptance, containment...).

Due to convolution of flux and cross-section, even if high correlation between flux, cross-section, and detector efficiency between near and far detector it will not remove all dependence.

The event rate approximately can be written as :

$$
\frac{\mathrm{N}_{\nu_{\beta}}^{F D}}{\mathrm{~N}_{\nu_{\alpha}}^{N D}} \approx \frac{\phi_{\nu_{\beta}}^{F D}\left(\mathrm{E}_{\nu}\right)}{\phi_{\nu_{\alpha}}^{N D}\left(\mathrm{E}_{\nu}\right)} \times \frac{\sigma_{\nu_{\beta}}^{F D}\left(\mathrm{E}_{\nu}\right)}{\sigma_{\nu_{\alpha}}^{N D}\left(\mathrm{E}_{\nu}\right)} \times \frac{\epsilon_{\nu_{\beta}}^{F D}}{\epsilon_{\nu_{\alpha}}^{N D}} \times \mathrm{P}_{\nu_{\alpha} \rightarrow \nu_{\beta}}\left(\mathrm{E}_{\nu}\right)
$$

The oscillated flux at the far detector is not same as the un-oscillated flux measured at near detector as far detector sees a point source of neutrino beam, whereas near detector sees a broadband of neutrino source. Also, the acceptance of particles is different at both the near and far detectors, efficiency calculation depends on the simulated particles from an event generator which is based on the neutrino-nucleus interaction model. The dependence of efficiency on the kinematics of the final state which is driven by cross-section and the different model predicts different particle multiplicity in the final state which gives birth to uncertainty. The uncertainty does not cancel exactly due to the difference in the near and far detectors. 


\subsection{Neutrino Kinematics}

Before moving to various neutrino-nucleus interactions, we need to familiarize with the neutrino kinematics notations, which have widespread use throughout this dissertation. In general, neutrino interacts with a target nucleus which produces a lepton or neutrino depending on the interaction type (charged current or neutral current), and a messy final-state particle (Fig. 2.1).

Let us consider the four-momentum of incoming neutrino to be $k\left(E_{\nu}, \vec{p}_{\nu}\right)$ and the out going leptons be $k^{\prime}\left(E_{l}, \vec{p}_{l}\right)$, the target nucleus four-momentum $p\left(E_{N}, \vec{p}_{N}\right)$ and the final-state hadrons $p^{\prime}\left(E_{H}, \vec{p}_{H}\right) . q$ is the four-momentum transferred between the neutrino-lepton system and the square of this transfer $\left(q^{2}\right)$ is Lorentz invariant and usually used as $Q^{2}=-q^{2}$ which determines what final-states are available to an interaction and mostly depends on the neutrino energy.

$$
\begin{aligned}
q^{2} & =\left(k^{\prime}-k\right)^{2} \\
& =\left(E_{l}-E_{\nu}\right)^{2}-\left(\vec{p}_{l}-\vec{p}_{\nu}\right)^{2} \\
& =-Q^{2} .
\end{aligned}
$$

The energy transfer will be $\nu=E_{\nu}-E_{l}$.

The fraction of the initial neutrino's energy transferred by the interaction is defined as inelasticity (y) and represented as

$$
\begin{aligned}
y & =\frac{p \cdot q}{p \cdot k} \\
& =\frac{E_{\nu}-E_{l}}{E_{\nu}} .
\end{aligned}
$$

The fraction of the target nucleon's momentum carried by the quark is termed as Bjorken scaling variable $(\mathrm{x})$ :

$$
\begin{aligned}
x & =\frac{Q^{2}}{2 p \cdot q} \\
& =\frac{Q^{2}}{2 M_{T} \nu},
\end{aligned}
$$

where $M_{T}$ is mass of the target nucleus.

The total invariant mass of the outgoing final-state particles is represented by $\mathrm{W}$ and can be written as:

$$
\begin{aligned}
W^{2} & =(p+q)^{2} \\
& =M_{T}^{2}+2 M_{T} E_{H}-Q^{2},
\end{aligned}
$$

where $E_{H}$ is mass of the hadrons. 


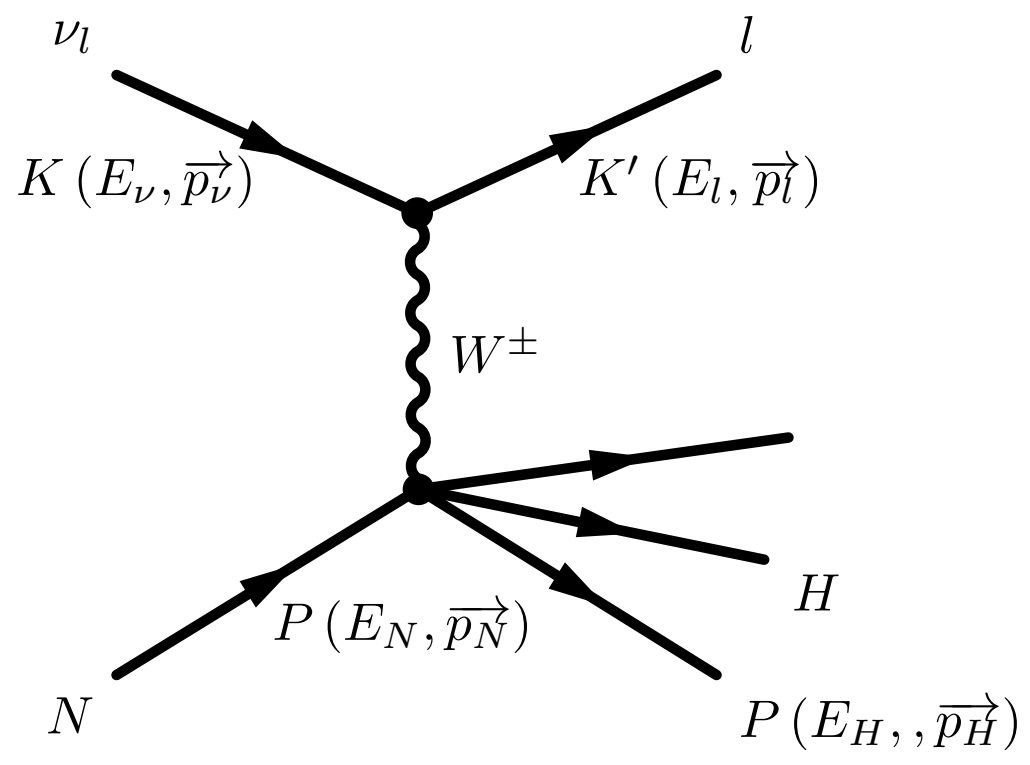

Figure 2.1: The standard neutrino interaction with a target, exiting with a (charged or neutral) lepton and a messy hadronic final-state particles.

\subsection{Neutrino Nucleus Interaction}

Neutrino-nucleus interaction largely depends on the energy of the neutrino, higher is the energy of neutrino better it can reach the small scale of length. We are dealing with two types of interactions such as charged current (CC) and neutral current (NC) interactions. The interaction process in which neutrino in and charge lepton out through the exchange of a charged $(W)$ boson. The flavor of outgoing lepton "tags" flavor of incoming neutrino and the charge of outgoing lepton determines whether it is neutrino or anti-neutrino (Fig. 2.2). In the NC process, the neutrino is both in the initial and final state since it is mediated by a neutral boson $(Z)$. It is impossible to reconstruct kinematics of final state particles in $\mathrm{NC}$ interactions, and these typically appear in oscillation measurements as backgrounds.
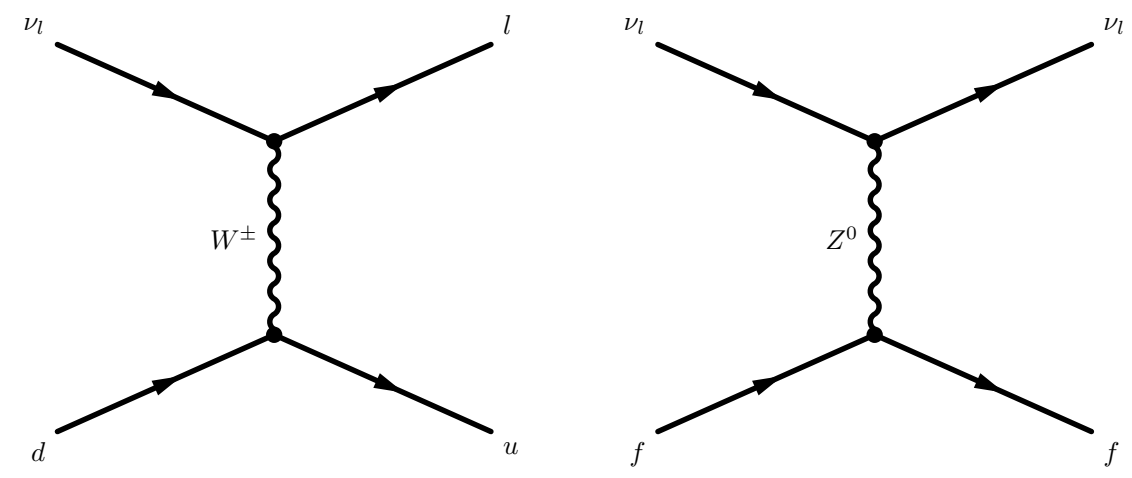

Figure 2.2: Charged (left) and Neutral Current (right) neutrino-nucleus interaction process. 
The inclusive muon neutrino charged-current analysis means, at least one muon will be in the final state particles irrespective of particular interaction process in which it takes care of summing over all possible nuclear final states.

\subsubsection{Quasi-elastic Interaction (QE)}

The neutrino scatters elastically off the nucleon ejecting a nucleon from the target and a charged lepton in the final state with an exchange of $W$ boson in the charge current process whereas, in the anti-neutrino scattering, a proton is converted into a neutron.

$$
\begin{gathered}
\nu_{\mu} n \rightarrow \mu^{-} p, \\
\bar{\nu}_{\mu} p \rightarrow \mu^{+} n
\end{gathered}
$$

In the charged current interaction process the "quasi" term arises because neutrino changes its identity to a charged lepton and the neutron can change into a proton by switching from $d$ quark to $u$ quark. In general, the quasi-elastic term refers to the events those are close to the quasi-elastic peak in lepton-kinematics. However, for a neutral current process, it is simply termed as elastic scattering.

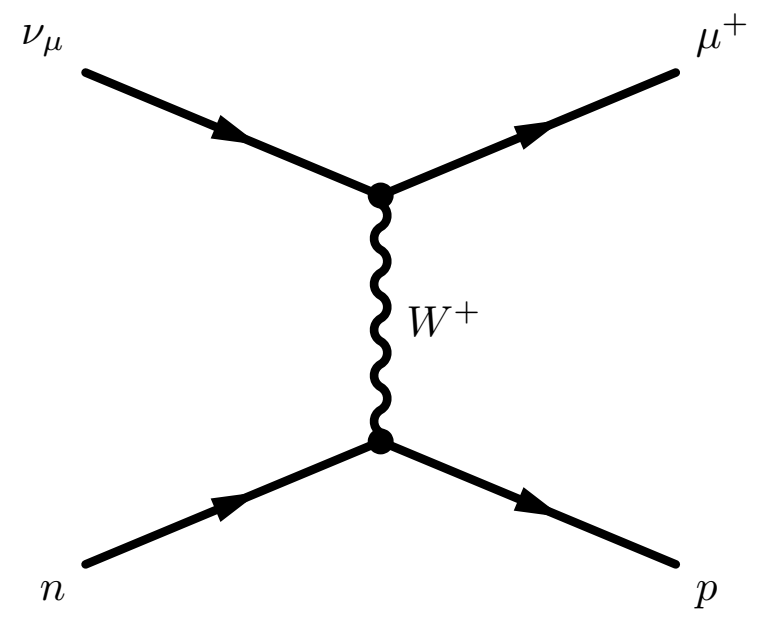

Figure 2.3: Quasi-elastic charged current neutrino nucleus interaction process.

QE interaction plays a central role in the neutrino energy of $\sim 2 \mathrm{GeV}$ and commonly used as a signal events in many neutrino oscillation experiments operating in this energy range. In this process, a muon can be clearly detected in the final state.

According to Llewellyn-Smith formalism [24] neutrino-nucleon quasi-elastic cross-section is expressed as:

$$
\frac{d \sigma}{d Q_{Q E}^{2}}\left(\begin{array}{l}
\nu_{l} n \rightarrow l^{-} p \\
\bar{\nu}_{l} p \rightarrow l^{+} n
\end{array}\right)=\frac{M^{2} G_{F}^{2} \cos ^{2} \theta_{c}}{8 \pi E_{\nu}^{2}}\left\{A\left(Q^{2}\right) \mp B\left(Q^{2}\right) \frac{s-u}{M^{2}}+C\left(Q^{2}\right) \frac{(s-u)^{2}}{M^{4}}\right\}
$$

where sign on B term is negative for neutrinos, positive for anti-neutrinos. $G_{F}$ is the Fermi constant $\left(1.17 \times 10^{-5} \mathrm{GeV}^{2}\right) . \mathrm{M}$ is the average nucleon mass $(939 \mathrm{MeV}) . \theta_{C}$ is the Cabbibo angle $\left(\cos \theta_{C}=\right.$ 
0.9742). $\mathrm{s}$ and $\mathrm{u}$ are Mandelstam variables. $E_{\nu}$ the incoming neutrino energy. A, B and $\mathrm{C}$ are combinations of hadronic form factors.

$$
\begin{aligned}
A\left(Q^{2}\right)= & \frac{m_{\mu}^{2}+Q^{2}}{M^{2}}\left\{\left(1+\frac{Q^{2}}{4 M^{2}}\right) F_{A}^{2}-\left(1-\frac{Q^{2}}{4 M^{2}}\right) F_{1}^{2}\right. \\
& +\frac{Q^{2}}{4 M^{2}}\left(1-\frac{Q^{2}}{4 M^{2}}\right)(\xi F)^{2}+\frac{Q^{2}}{M^{2}} \operatorname{Re}\left(F_{1}^{*} \xi F_{2}\right)-\frac{Q^{2}}{M^{2}}\left(1+\frac{Q^{2}}{4 M^{2}}\right)\left((F)_{A}^{3}\right)^{2}- \\
& \left.\frac{\mu^{2}}{4 M^{2}}\left[\left|F_{1}+\xi F_{2}\right|^{2}+\left|F_{1}+2 F_{p}\right|^{2}-4\left(1+\frac{Q^{2}}{4 M^{2}}\right)\left(\left((F)_{A}^{3}\right)^{2}+F_{p}^{2}\right)\right]\right\}, \\
B\left(Q^{2}\right)= & \frac{Q^{2}}{M^{2}} \operatorname{Re}\left[F_{A}^{*}\left(F_{1}+\xi F_{2}\right)\right]-\frac{\mu^{2}}{M^{2}} \operatorname{Re}\left[\left(F_{1}-\tau \xi F_{2}\right) F_{V}^{3 *}-\left(F_{A}^{*}-\frac{Q^{2}}{2 M^{2}} F_{p}\right) F_{A}^{3}\right], \\
C\left(Q^{2}\right)= & \frac{1}{4}\left\{F_{A}^{2}+F_{1}^{2}+\tau\left(\xi F_{2}\right)^{2}+\frac{Q^{2}}{M^{2}}\left(F_{A}^{3}\right)^{2}\right\} .
\end{aligned}
$$

Out of six form factors two are vector $\left(F_{1}\right.$ and $\left.F_{2}\right)$, an axial vector $\left(F_{A}\right)$, a pseudo-scalar $\left(F_{P}\right)$, and two small second-order terms $\left(F_{A}^{3}\right.$ and $\left.F_{V}^{3}\right)$.

The vector form factors $\left(F_{1}\right.$ and $\left.F_{2}\right)$ can be expressed considering the conserved vector current hypothesis $(\mathrm{CVC})[25,26]$ in terms of the form factors

$$
\begin{gathered}
F_{1}\left(Q^{2}\right)=\frac{G_{E}^{V}\left(Q^{2}\right)+\frac{Q^{2}}{4 M^{2}} G_{M}^{V}\left(Q^{2}\right)}{1+\frac{Q^{2}}{4 M^{2}}} . \\
\xi F_{2}\left(Q^{2}\right)=\frac{G_{M}^{V}\left(Q^{2}\right)-G_{E}^{V}\left(Q^{2}\right)}{1+\frac{Q^{2}}{4 M^{2}}}
\end{gathered}
$$

where $G_{M}^{V}$ and $G_{E}^{V}$ are the electron scattering form factors and represented by

$$
\begin{aligned}
G_{E}^{V}\left(Q^{2}\right) & =G_{E}^{p}\left(Q^{2}\right)-G_{E}^{n}\left(Q^{2}\right), \\
G_{M}^{V}\left(Q^{2}\right) & =G_{M}^{p}\left(Q^{2}\right)-G_{M}^{n}\left(Q^{2}\right),
\end{aligned}
$$

where $G_{E}^{p}$ and $G_{E}^{n}$ are the electric form factors for the proton and neutron, $G_{M}^{p}$ and $G_{M}^{n}$ are the magnetic form factors of the proton and neutron. The behavior of form factor changes according to $Q^{2}$ and it can be measured through electron scattering experiments. When $Q^{2}=0$, the electric form factors correspond to the electric charge of the proton (1) and neutron (0), however magnetic form factors correspond to the magnetic moments of proton and neutron. At $Q^{2}>0$, the form factors are determined via dipole approximation and nucleons charge is distributed exponentially 
$\rho(r)=\rho_{0} e^{-M r}$ and the dipole form factor can be obtained from Fourier transformation as:

$$
\begin{aligned}
G_{D}\left(Q^{2}\right) & =\frac{1}{\left(1+\frac{Q^{2}}{4 M_{V}^{2}}\right)^{2}}, \\
G_{E}^{p}\left(Q^{2}\right) & =G_{D}\left(Q^{2}\right) \\
G_{E}^{n}\left(Q^{2}\right) & =0 \\
G_{M}^{p}\left(Q^{2}\right) & =\mu_{p} G_{D}\left(Q^{2}\right) \\
G_{M}^{n}\left(Q^{2}\right) & =\mu_{n} G_{D}\left(Q^{2}\right)
\end{aligned}
$$

where $\mathrm{M}$ is the mass of nucleon, $M_{V}$ is the vector mass $=0.843 \mathrm{GeV} \cdot \mu_{p}=2.79$ and $\mu_{n}=-1.91$ are the magnetic moment of the proton and neutron respectively.

The pseudo-scalar form factor $\left(F_{V}\right)$ is related with axial form factor $\left(F_{A}\right)$ and can be expressed as

$$
\begin{aligned}
& F_{P}\left(Q^{2}\right)=\frac{2 M^{2}}{Q^{2}+m_{\pi}^{2}} F_{A}\left(Q^{2}\right), \\
& F_{A}\left(Q^{2}\right)=\frac{g_{A}}{\left(1+\frac{Q^{2}}{M_{A}^{2}}\right)^{2}},
\end{aligned}
$$

$F_{A}$ is parameterized as dipole. The average axial mass constant $M_{A}=1.014 \pm 0.014 \mathrm{GeV} / \mathrm{c}^{2}$ was extracted by Bodek et al. [27] and the best axial vector constant $g_{A}=1.267$ and it is know from beta decay experiments. The more details on $M_{A}$ can be found in Ref. [28].

\subsubsection{Resonance Pion Production (Res)}

In this process, meson production in the final state through excitation of the nucleon in to a resonant state. The resonant states are unstable and further decay in to mesons and nucleons, of course a charged lepton produced in case of charge current interaction (Fig. 2.4). It is more active at the invariant mass $\mathrm{W}<2 \mathrm{GeV}$ and more contribution coming in the range of $0.5-3 \mathrm{GeV}$ energy of neutrino. Intermediate resonance state is $\Delta_{33}$ (1232) in the lower neutrino energy which produces single pions and in the higher neutrino energy range the higher mass resonances are $P_{11}$ (1440), $S_{11}$ (1535) and $D_{13}$ (1520) which decay to multiple pions, kaons, and photons in addition to single pions. Example of different channels of charge current and neutral current resonance processes are:

$$
\begin{gathered}
\nu_{\mu} n \rightarrow \mu^{-} n \pi^{+}, \bar{\nu}_{\mu} p \rightarrow \mu^{+} p \pi^{-} \\
\nu_{\mu} n \rightarrow \mu^{-} p \pi^{0}, \bar{\nu}_{\mu} p \rightarrow \mu^{+} n \pi^{-} \\
\nu_{\mu} p \rightarrow \mu^{-} p \pi^{+}, \bar{\nu}_{\mu} n \rightarrow \mu^{+} n \pi^{0} \\
\nu_{\mu} p \rightarrow \nu_{\mu} p \pi^{0}, \bar{\nu}_{\mu} p \rightarrow \bar{\nu}_{\mu} p \pi^{0} \\
\nu_{\mu} n \rightarrow \nu_{\mu} n \pi^{0}, \bar{\nu}_{\mu} n \rightarrow \bar{\nu}_{\mu} n \pi^{0} \\
\nu_{\mu} p \rightarrow \nu_{\mu} n \pi^{+}, \bar{\nu}_{\mu} p \rightarrow \bar{\nu}_{\mu} n \pi^{+} \\
\nu_{\mu} n \rightarrow \nu_{\mu} p \pi^{-}, \bar{\nu}_{\mu} n \rightarrow \bar{\nu}_{\mu} p \pi^{-}
\end{gathered}
$$

The double differential cross-section for a single meson production is given by: 


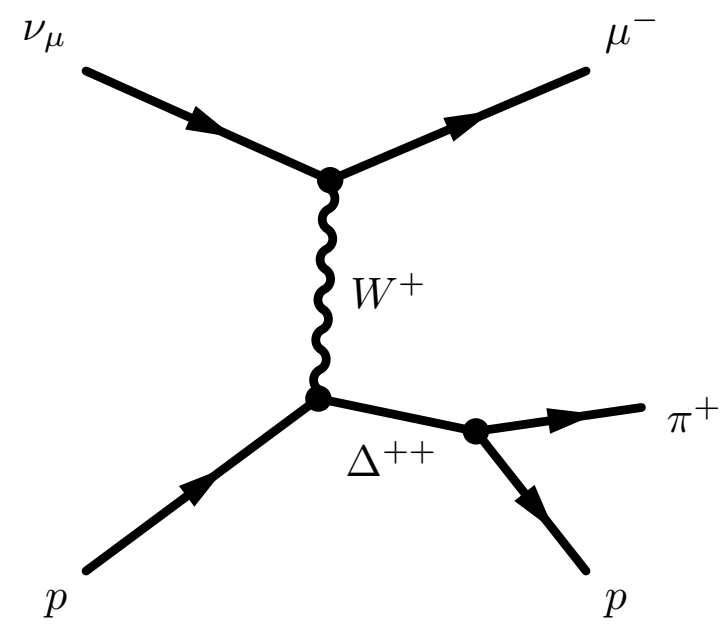

Figure 2.4: Charged current resonance neutrino nucleus interaction process.

$$
\frac{d^{2} \sigma}{d Q^{2} d \nu}=\frac{1}{32 \pi M E_{\nu}^{2}} \frac{1}{2} \sum_{\text {spins }}\left|T\left(\nu N \rightarrow l N^{*}\right)\right|^{2} \delta\left(W^{2}-M^{2}\right)
$$

where $\mathrm{M}$ is the mass of the initial state of nucleon, $\nu$ is the energy transfer, $T\left(\nu N \rightarrow l N^{*}\right)$ is the amplitude of a given resonance production and $\mathrm{W}$ is the hadronic invariant mass. The amplitude is calculated using FKR model [29].

\subsubsection{Coherent Scattering (Coh)}

Coherent scattering is a process by which a neutrino interacts with a nucleus, the nucleus is not excited to a higher energy state and coherently scatters off target nucleus with small exchange of energy between nucleus and neutrino with single meson (pions, kaons, rho) in the final state (Fig. 2.5). The charge current and neutral current coherent processes are:

$$
\begin{gathered}
\nu_{\mu} A \rightarrow \mu^{-} A \pi^{+}, \bar{\nu}_{\mu} A \rightarrow \mu^{+} A \pi^{-} \\
\nu_{\mu} A \rightarrow \nu_{\mu} A \pi^{0}, \bar{\nu}_{\mu} A \rightarrow \bar{\nu}_{\mu} A \pi^{0}
\end{gathered}
$$

Coherent interaction process has important role on the $\nu_{\mu} \rightarrow \nu_{e}$ oscillation analysis. As the final state has $\pi^{0}$ which decays to two photons if any one of the photons is missing from this sample which can be misidentified as $\nu_{e}$ signal event. This might occurs either missed photon exits the detector before showering or does not have enough energy to produce a shower. Also the misidentification of charged current coherent $\pi^{+}$as proton which disturbs in reconstructing the neutrino energy in particularly $\nu_{\mu}$ disappearance analysis.

The coherent scattering process occurs at low- $Q^{2}$ region and it requires a small momentum transfer to the target nucleus. According to partially conserved axial current (PCAC) hypothesis, the differential cross-section at $Q^{2}=0$ is given by: 


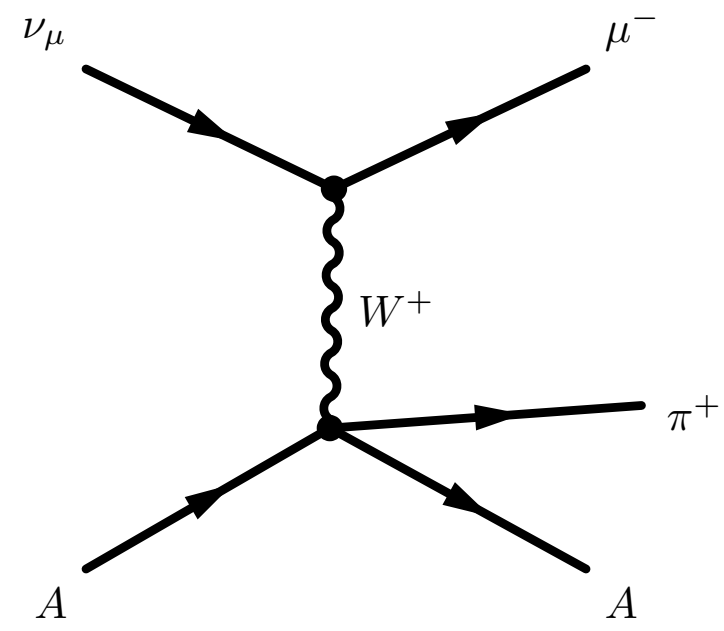

Figure 2.5: Charged current coherent neutrino-nucleus interaction process.

$$
\left.\frac{d^{3} \sigma(\nu A \rightarrow l A \pi)}{d x d y d Q^{2}}\right|_{Q^{2}=0}=\left.\frac{G_{F}^{2}}{\pi^{2}} f_{\pi}^{2} M E_{\nu}(1-y) \frac{\sigma(\pi A \rightarrow \pi A)}{d Q^{2}}\right|_{E_{\nu} y=E_{\pi}},
$$

where $\mathrm{x}, \mathrm{y}$ are Bjorken scaling variables, $G_{F}$ is the weak coupling constant, $f_{\pi}$ is the pion decay constant, $\mathrm{M}$ is the mass of the nucleon and $Q^{2}$ is the square of the four-momentum transferred to the target nucleus.

At $Q^{2} \neq 0$, the cross-section is dipole dependent and the cross-section is calculated from measured data on total and inelastic pion scattering from protons and deuterium.

\subsubsection{Deep-inelastic Scattering (DIS)}

In this interaction process, the neutrino scatters off a quark in the nucleon producing a messy hadronic system in the final state (Fig. 2.6). As the neutrino has enough energy to interact at the quark level in the nucleons, so it is termed as "deep". Beyond resonance interaction range, DIS interaction range is at the invariant mass $\mathrm{W} \geq 2 \mathrm{GeV}$ and at high momentum transfer of $Q^{2} \geq 1$ $\mathrm{GeV}^{2}$.

The intermediate state between resonance process and DIS process is called as shallow inelastic scattering (SIS). In the DIS interaction, as it is occurring in the high neutrino energy, most of the energy transferred to the hadronic system and small energy goes to the lepton, so we detect a short track length of muon. Example of charge current and neutral current DIS interaction processes are:

$$
\begin{aligned}
& \nu_{\mu} N \rightarrow \mu^{-} X, \bar{\nu}_{\mu} N \rightarrow \mu^{+} X \\
& \nu_{\mu} N \rightarrow \nu_{\mu} X, \bar{\nu}_{\mu} N \rightarrow \bar{\nu}_{\mu} X,
\end{aligned}
$$

where $\mathrm{N}$ is nucleon bound inside a nucleus and $\mathrm{X}$ is the hadrons in the final state. 


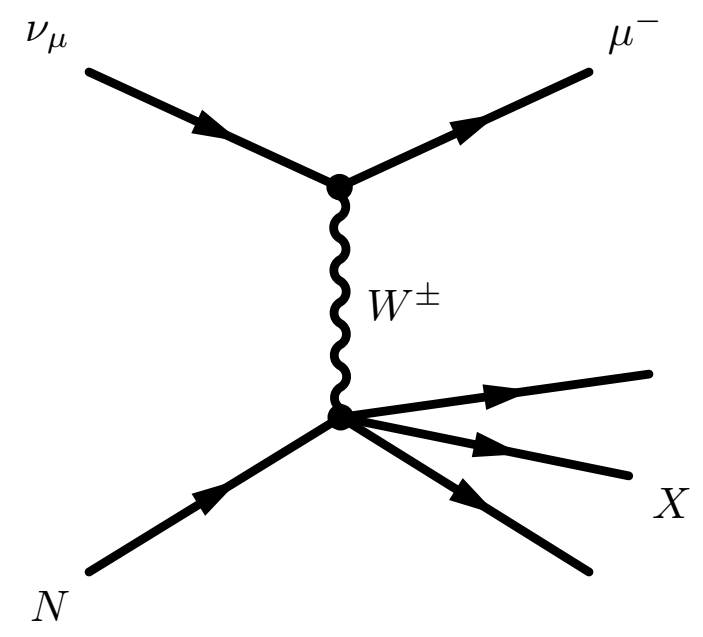

Figure 2.6: Charged current DIS neutrino nucleus interaction process.

The double-differential cross-section for this interaction is determined as follows:

$$
\frac{d^{2} \sigma}{d x d y}=\frac{G_{F}^{2} M E_{\nu}}{\pi}\left[\left(1-y+\frac{y^{2}}{2}+C_{1}\right) F_{2}(x) \pm y\left(1-\frac{y}{2}+C_{2}\right) x F_{3}(x)\right],
$$

where $\mathrm{x}, \mathrm{y}$ are the Bjorken scaling variable as defined in the 2.4 and $C_{1}, C_{2}$ depend on these variables and defined as:

$$
\begin{aligned}
C_{1} & =\frac{M_{l}^{2}(y-2)}{4 M E_{\nu} x}-\frac{M x y}{2 E_{\nu}}-\frac{M_{l}^{2}}{4 E_{\nu}^{2}}, \\
C_{2} & =-\frac{M_{l}^{2}}{4 M x E_{\nu}},
\end{aligned}
$$

where $M_{l}$ and $\mathrm{M}$ is mass of the final-state lepton and nucleons respectively. The $F_{2}(x), x F_{3}(x)$ are the nucleon structure functions calculated using parton distribution function [30].

\subsubsection{Meson Exchange Current (MEC)}

There is another process beyond above processes currently draws more attention in the neutrino scattering community. In this process, when neutrino communicates with nucleons through $W$ it gets absorbed by nucleons and knocking out two-nucleon and two hole pairs (2p-2h) through an exchange of a meson (Fig. 2.7).

There is also a possibility of producing $3 \mathrm{p}-3 \mathrm{~h}$ in the final state, so overall this is called np-nh or multi-nucleon excitation. Nowadays excitation of multi-nucleon and charged lepton in the final state except the pion absorption is termed as quasielastic-like events. Excitation of $2 \mathrm{p}-2 \mathrm{~h}$ was first proposed by Delorme and Ericson in the neutrino scattering process [31]. This process has significant contribution in the neutrino-nucleus scattering and the community agrees that meson exchange currents are needed to explain the neutrino QE data. However, there are several phenomenological approaches by Lalakulich, Mosel et. al. [32, 33, 34, 35] and Bodek et. al. [36] and the most competitive theoretical approach by Martini et. al [37, 38, 39, 40, 41], Nieves et. al. [42, 43, 44, 45, 46] 


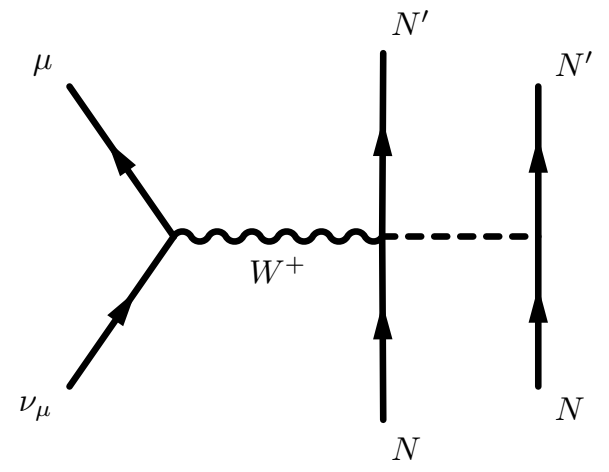

Figure 2.7: Charged current meson exchange current neutrino-nucleus interaction process.

and Amaro et. al. [47, 48, 49, 50, 51, 52, 53, 54] to address the np-nh contributions to neutrinonucleus cross-section.

\subsection{Nuclear Effects}

Almost all modern neutrino detectors are made of heavy nuclei. Much of the uncertainty on crosssection models is due to poorly understood nuclear effects. This systematic uncertainty is one of the biggest uncertainties for the oscillation experiments such as NOvA, T2K and future long-baseline experiments DUNE and Hyper-Kamiokande. In these accelerator-based experiments, the neutrino beam energy ranges from hundreds of $\mathrm{MeV}$ to a few $\mathrm{GeV}$. Using such neutrino energies, a neutrino can probe the structure of the nucleus at the nucleon level. The effects of the nuclear environment on neutrino interactions are substantial and some of them are discussed below.

\subsubsection{Relativistic Fermi Gas Model (RFG)}

According to the Pauli exclusion principle, no two identical fermions can occupy the same state. In nuclei, there will be many protons and neutrons which are distributed by Fermi-Dirac statistics. Nucleons occupy states in Fermi gas (all the nucleons are non-interacting and all states are filled up the same order) up to Fermi level. In the Fermi gas model, nucleons move independently within the nuclear volume in a constant binding potential generated by all nucleons which are known as Fermi motion and their energy in the potential gives them Fermi momentum $\left(k_{F}\right)$. Another term called Pauli blocking is related to Relativistic Fermi Gas model, in which nucleons cannot achieve the momentum state that is already occupied, so it should have a momentum above Fermi momentum. When an interaction with a neutrino transfer enough energy to put a nucleon above the Fermi level and binding energy, nucleon gets ejected and rest of the nucleons remains as a spectator, otherwise, it is Pauli-blocked and the reaction is suppressed, and the kinematic distributions are modified due to Pauli blocking.

In general, the above approach of Relativistic Fermi Gas model is known as Global Fermi Gas (GFG) model. In the case of GFG, the nucleus is assumed as a box of constant nuclear density and the momentum distribution is the size of the nucleus, defined as : 

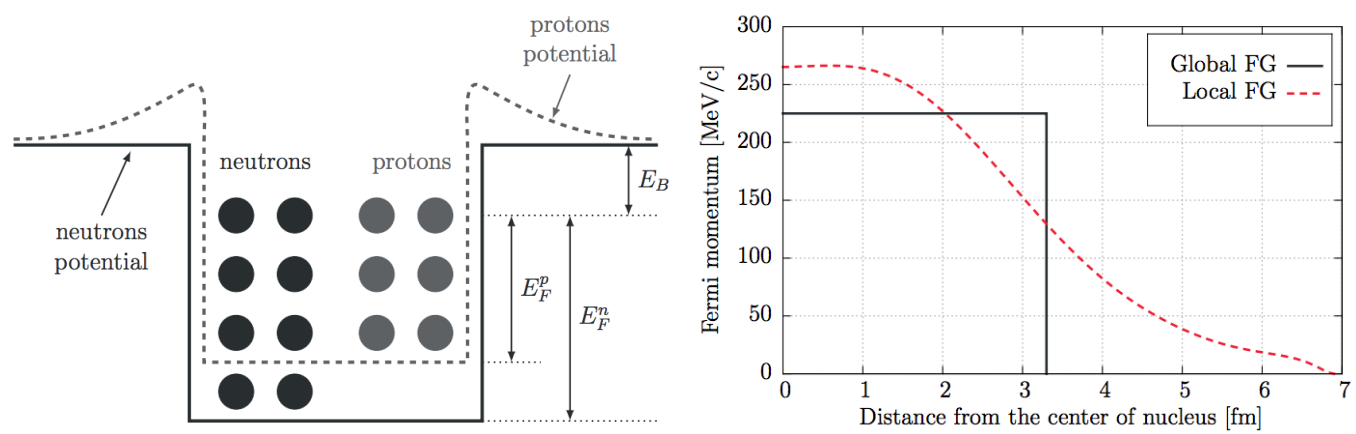

Figure 2.8: Cartoon of a potential wall (left) and a compasion of Local Fermi Gas and Global Fermi Gas model (right). Both figures are reprinted from [55] .

$$
p_{F}^{G F G}=\frac{\hbar}{r_{0}}\left(\frac{9 \pi N}{4 A}\right)^{1 / 3}
$$

where $\mathrm{N}(\mathrm{A}-\mathrm{Z})$ is the number of neutrons and for proton, the momentum distribution is replaced by $\mathrm{Z}$ in the place of $\mathrm{N}$. $\mathrm{A}$ is the atomic mass and $R=r_{0} A^{1 / 3}$ in the radius of Fermi sphere (the energy state occupied by nucleons with momentum space in the sphere) and the value of $r_{0}=1.25 \pm 0.20$ fm.

The minimal extension of RFG model is called Local Fermi Gas (LFG) model where Fermi momentum and binding energy (the amount of energy needed to exit the nucleon from the nucleus) depends on the radial position in the nucleus, following the density profile of the nuclear matter.

$$
p_{F}^{L F G}=\hbar\left(3 \pi^{2} \rho(r) \frac{N}{A}\right)^{1 / 3},
$$

where $\rho(r)$ is nuclear density depends on the radius (distance from the center of the nucleus). This distribution arises from the electron scattering data [56]. The comparative distribution of LFG and GFG is shown in Figure 2.8. Use of Local Fermi Gas model will affect the final state nucleons energy distribution [57] as well as the cross-section.

\subsubsection{Short Range Correlation (SRC)}

The Relativistic Fermi Gas model is used to describe the nuclear effects in the QE region, which is based on the simplest independent particle model called Fermi Gas model. Here all the nucleons in the nucleus are degenerate, or in other word nucleons are uncorrelated. If we think of nucleons in the nucleus are correlated, and the distance between two nucleons are very short $(<15 \mathrm{fm})$ there is an involvement of a mechanism called short-range correlation [58] in which strong short-range forces get to increase their relative momentum (more than Fermi momenta) and impel the two nucleons far off-shell. A nucleon involvement in this process is about $20 \%$ of the time and most of the time participation in correlation is proton-neutron $[59,60]$.

Short-range correlation generates two high energetic isosinglet nucleons with opposite isospins and an isotriplet component which can contribute to proton-proton, proton-neutron, and neutronneutron pairs. More details can be found in Refs. [28]. 


\subsubsection{Random Phase Approximation (RPA)}

The Random Phase Approximation (RPA) method has been developed to understand the longrange correlation between nucleons in the nucleus, also it outlines the collective excitation of the nucleus. The collective excitations of different momenta are orthogonal and the phases can be treated randomly, so it is termed as "Random Phase". The RPA is a non- perturbative method to describe the complexity of many-body interactions. So far RPA has been studied in the QE regime where there is knockout of a single nucleon and one of the great success is the prediction of collective surface vibrations, called giant resonances in the energy range is in between 0.015 and $0.030 \mathrm{GeV}$ [61].

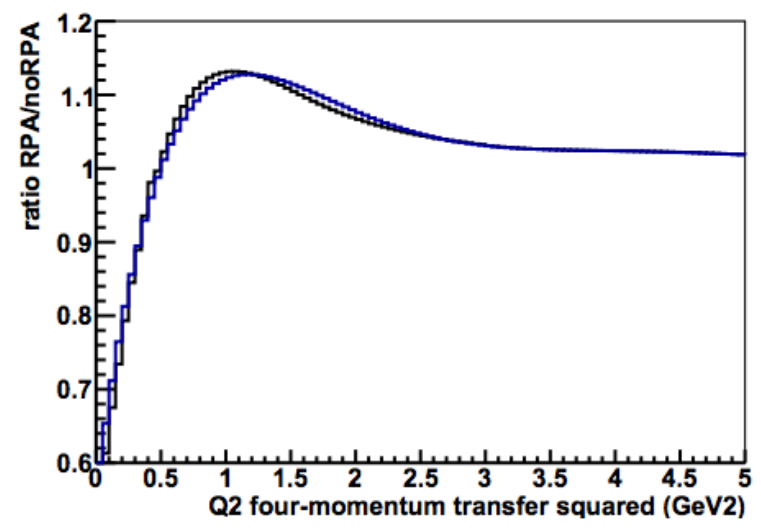

Figure 2.9: Ratio of RPA/noRPA for GENIE neutrino events with the full central-weighting as a function of four-momentum transfer squared. This figure is reprinted from Ref. [62].

RPA also considers the modification of the electroweak coupling strengths, due to the presence of strongly interacting nucleons in the nucleus in comparison to its free nucleon coupling strength [63]. RPA corrections strongly decrease as the neutrino energy increases. RPA is strongly suppressed at low four-momentum transfer (it shows that nucleons embedded in nuclear potential) also there is mild enhancement at higher four-momentum transfer (almost no RPA effect as $Q^{2}$ increasing, which means if high energy transferred to the nucleus than nucleons, quarks might be free) is shown in Fig. 2.9). A variant of RPA, so-called continuum RPA takes into account that the final nucleon is in the continuum.

\subsubsection{Final State Interactions (FSI)}

Initially, when a neutrino interacts with one of nucleon it generates hadrons (pions, protons etc.) with ejecting a final state charged lepton exiting from the nucleus. The hadronic shower again scatters and re-scatter with rest of the nucleons inside the dense nuclear matter before exiting the nucleus. This changes the kinematics, multiplicity, and charge of the hadrons in the final state which is described in Fig. 2.10 [64].

FSI effects on the topology of events reconstruction. For example, if we see a muon and proton in the final state and no pions in the detector, we can not tell if this was a "real" CCQE events or an RES event where the pion has been reabsorbed in the nucleus. It resulted in CC-RES events showed up as $\mathrm{CCQE}-$ like $(\mathrm{CC} 0 \pi)$ events. 


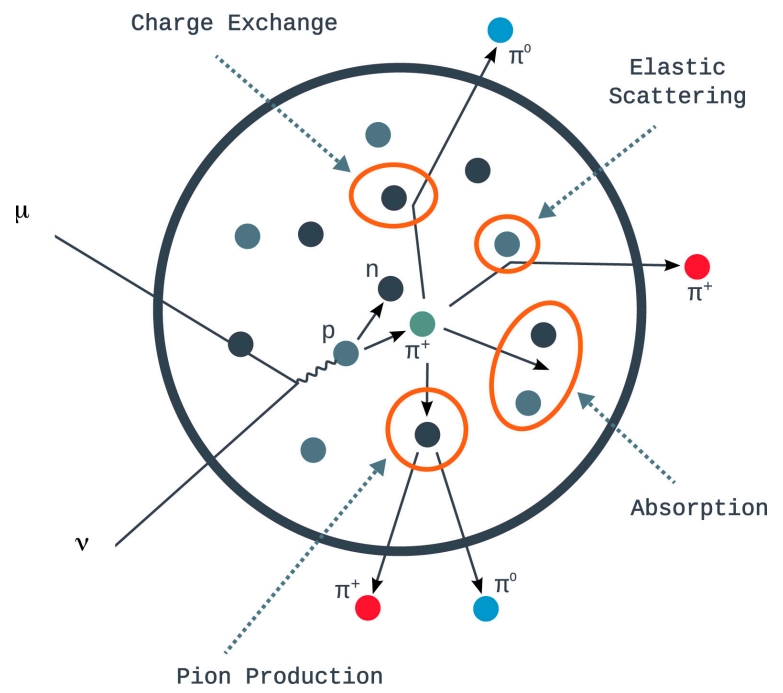

Figure 2.10: Carton of a final state interaction reprinted from Ref. [64].

One more important thing is that neutrino energy is unknown when it is entering the nucleus. The neutrino energy is reconstructed from the final state particles what we observe in our detector. It is also occurring that some energy gets lost in the re-scattering in the nucleus and cannot be reconstructed. So FSI impact on the neutrino calorimetric energy reconstruction.

The FSI can be estimated by computing a mean free path for scattering $(\lambda)$ of the struck nucleon,

$$
\lambda(r)=[\sigma \rho(r)]^{-1}
$$

where $\rho(r)$ is the nuclear density, $\sigma$ is scattering cross-section between nucleon-nucleon. 


\section{Chapter 3}

\section{The NOvA Experiment}

The NuMI Off-axis $\nu_{e}$ Appearance or NOvA consists two detectors (near detector and far detector), a long baseline oscillation experiment which uses high-intensity neutrino source coming from Fermilab's Neutrinos at the Main Injector (NuMI) [65]. The aim of placing two detectors at a long distance is to observe neutrino oscillation. The near detector (ND) is placed $1 \mathrm{~km}$ downstream from the neutrino source, mainly used to measure the composition of the unoscillated beam as it is close to the source, neutrino did not get chance to oscillate. In addition to that, it provides a very good opportunity for cross-section measurement. The far detector is $810 \mathrm{~km}$ away from the neutrino source located at Ash River, Minnesota, which measure the oscillated neutrinos. Both detectors are functionally identical but vary in dimension. NuMI could produce both neutrinos and anti-neutrinos. The oscillation probability is different for neutrinos and anti-neutrinos as leading terms arises from matter effect. Comparing the appearance probability in the neutrino and anti-neutrino, it could be possible to determine the mass ordering is normal or inverted. Also the difference between oscillation probability on neutrino and anti-neutrino which leads to measure the charge-parity $(\mathrm{CP})$ violating phase $\delta$. This chapter describes NuMI beams and the NOvA detector design in details.

\subsection{NuMI Neutrino Beam}

\subsubsection{Fermilab Accelerator Complex}

The Fermilab's accelerator complex (shown in Fig 3.1) produces neutrino beam which is considered to be the most powerful at this moment, which is used by several neutrino experiments at Fermilab. Firstly, the negative hydrogen atoms are produced and accelerated to $0.4 \mathrm{GeV}$ of kinetic energy in the Linear Accelerator (LINAC). These negatively charged hydrogen atoms are converted into positively charged hydrogen atom (protons) after passing through a carbon foil before fed into the Booster ring and the protons are accelerated up to $8 \mathrm{GeV}$ of kinetic energy. The batches of a proton from the Booster are shoot into the Main Injector (MI) synchrotron accelerator, accelerates $120 \mathrm{GeV}$ protons in the $3.3 \mathrm{~km}$ of the circumference. The beam of protons are extracted from the MI and bent $58 \mathrm{mrad}$ downward in the direction of MINOS detectors.

Earlier NuMI beam was designed for MINOS on-axis neutrino experiment. In order to achieve the beam power up to $700 \mathrm{~kW}$, required for the goals of NOvA's physics program the accelerator complex at Fermilab had been upgraded. Beam power has been increased after reducing the cycle 


\section{Fermilab Accelerator Complex}

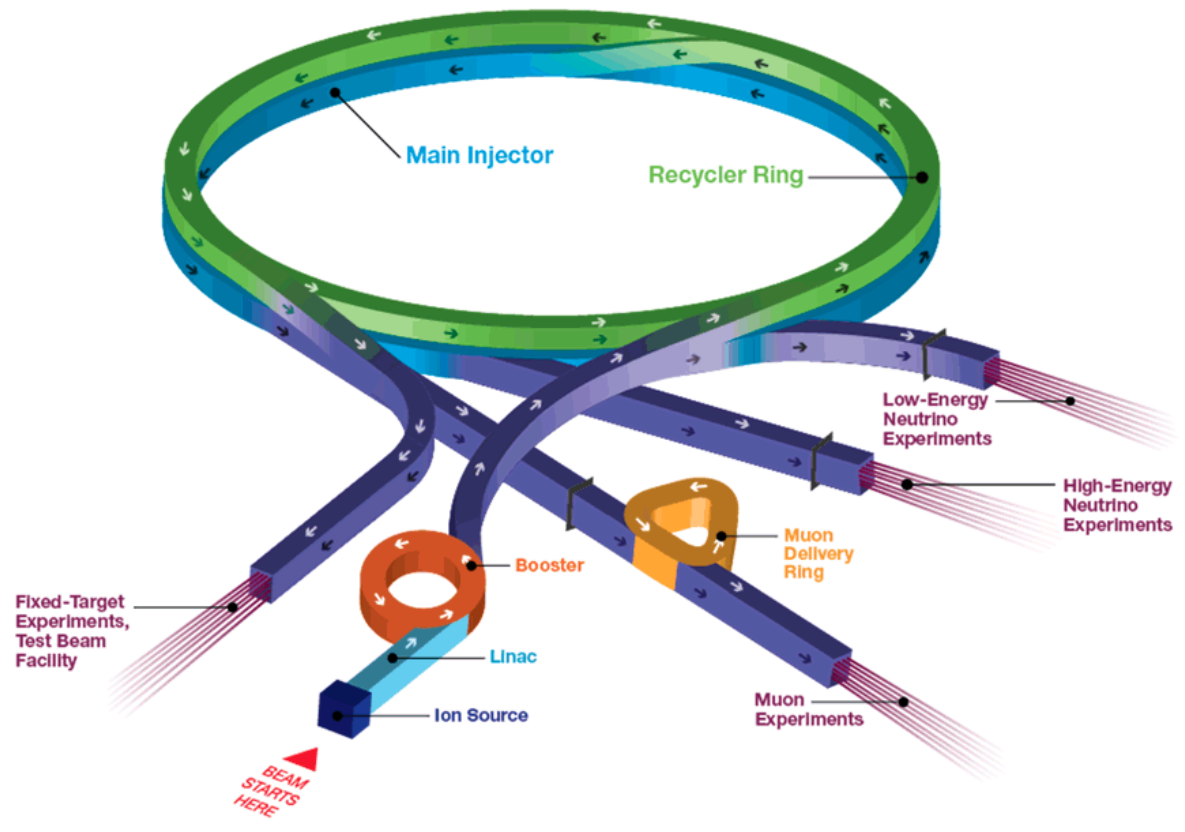

Figure 3.1: Femilab's accelerator complex

time from 2.2 seconds to 1.33 seconds in the MI and it was done by adding two radio frequency (RF) cavities to increase the rate of acceleration. From the Tevatron era, anti-proton storage ring has been transformed into the Recycler storage ring. The protons from Booster first inject to the Recycler and the Recycler transformed the protons into the MI. To increase the beam intensity of the NuMI beam there was an improvement on slip-stacking of all the proton batches in the Recycler. The detail descriptions can be viewed at NOvA TDR [66]. The first six proton bunches coming from the Booster enters to the Recycler and it is decelerated by reducing the RF pulse. Next, the six new bunches introduced to Recycler from Booster, now both the bunches have two different energy and they slip with reference to one another. At a tuned RF frequency the two bunches are overlapped and making a larger bunches before the next bunches introduced. Finally, these bunches are extracted into the MI and the intensity of each bunch or spill is $\sim 5 \times 10^{13}$ protons in a 10 microseconds beam window.

\subsubsection{NuMI Beamline}

The NuMI beamline starts at Fermilab, Batavia, IL which shoots towards the MINOS detector hall and reached out to Minnesota and finally exits the earth. Firstly $120 \mathrm{GeV}$ of protons (higher the energy of proton beams, produces high energetic mesons and further meson decays in to more energetic neutrino fluxes) from the Main Injector strikes to the segmented graphite target, it has 47 segmented rectangular pieces (fins) stacked together having $20.0 \mathrm{~mm}$ long and separated with a space of $0.3 \mathrm{~mm}$ makes a total length of $95.4 \mathrm{~cm}$ and the density of target is $1.78 \mathrm{~g} / \mathrm{cm}^{3}$. To generate more interaction of proton with the target we can increase the length of the target, however, it expands 


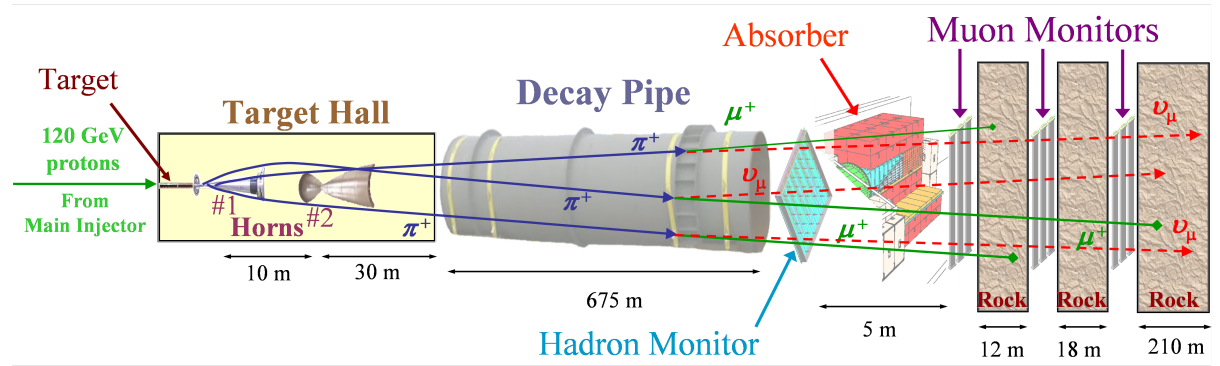

Figure 3.2: NuMI Beamline [67]

the scattering of the produced mesons and generates more interactions due to a more intense beam and makes a hotter target. In the era of NOvA target, water is circulated for cooling between two stainless steel pipe which encloses the fins. The hadrons exit from the target is focused through two parabolic shaped magnetic horns which cancel the transverse momentum of hadrons. The magnetic horn is a bi-layered coaxial conductor sheet made of with Aluminum filled with a low-density gas such as air, He or Ar. A $200 \mathrm{kA}$ current is passed through the horn and it creates a $1 / R$ magnetic field according to Amperes law and it focuses the charged particles of a particular sign and deflects the opposite sign. We can change our current according to our need for neutrino or anti-neutrino beam. If + vely charged particles get focused, they decay into a neutrino, however, anti-neutrinos can be achievable by focusing the -vely charged particles. Next, the focused charged particles are allowed to decay within the decay pipe length of $675 \mathrm{~m}$ long, $1 \mathrm{~m}$ radii where the hadron decay products are neutrinos and charged leptons. Ultimately, particles passing through the hadron monitor, a watercooled Aluminum hadron absorber is placed followed by three ionization chambers to monitor the muons, spaced between rocks, exiting only neutrinos run towards MINOS building. The schematic view of the NuMI beamline is shown in Fig. 3.2.

\subsubsection{Off-Axis Detectors}

Inside the decay pipe charged hadrons are decays and produces muon neutrinos through $: \pi^{+} \longrightarrow$ $\mu^{+}+\nu_{\mu}$ and $K^{+} \longrightarrow \mu^{+}+\nu_{\mu}$ having branching ratio $99.98 \%$ and $63.55 \%$ respectively. The decay of pions and kaons in the rest frame is isotropic which produces mono-energetic neutrinos, where as in the lab frame, using relativistic kinematics, the neutrino flux $(\Phi)$ can be shown as a function of angle, cross-section area of detector A located at a distance $L$ is :

$$
\Phi=\left(\frac{2 \gamma}{1+\gamma^{2} \theta^{2}}\right)^{2} \frac{A}{4 \pi L^{2}}
$$

The energy spectra of neutrino $\left(E_{\nu}\right)$ as a function of pion energy and an angle is given by

$$
E_{\nu}=\frac{0.43 E_{\pi}}{1+\gamma^{2} \theta^{2}}
$$

In the above equation, $\theta$ is the angle between outgoing neutrino and direction of meson, $\gamma=\frac{E_{\pi}}{m_{\pi}}$. The relationship between $E_{\nu}$ and $E_{\pi}$ for various off-axis angles are shown in Fig. 3.3. The red curve in the picture (shown in left Fig. 3.3) shows off-axis angle chosen for both NOvA detectors in order to optimize the sensitivity for the oscillation of muon neutrino to an electron neutrino. The choice of 


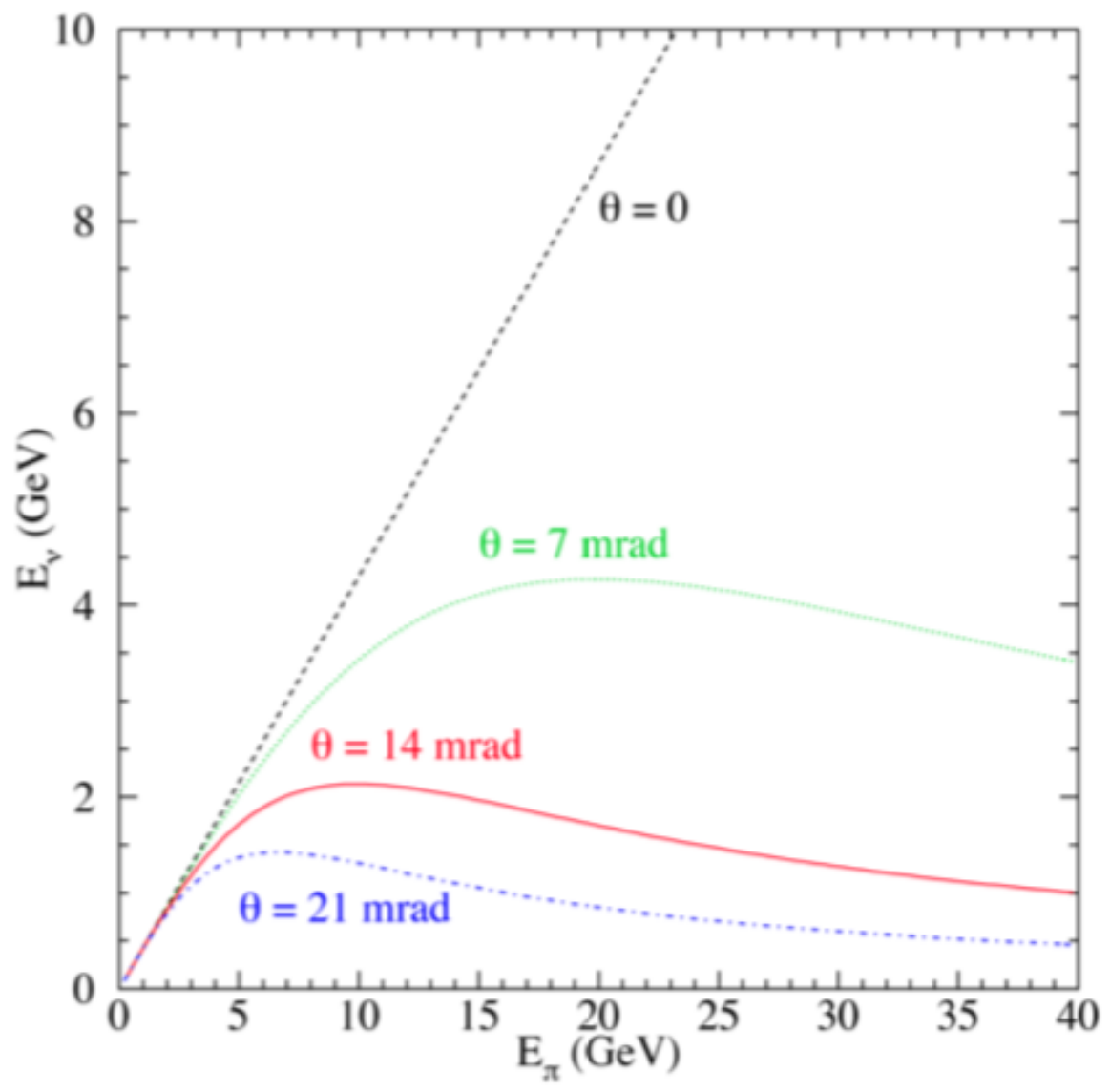

Figure 3.3: The relationship between neutrino energy and pion energy for different off-axis angles.

making 14.6 milliradians off-axis, NuMI beams generates a narrow band of neutrino energy spectra (shown in left Fig. 3.4). Due to off-axis angle, it also suppresses the neutral current events and increases the statistics of signal ( $\nu_{\mu}$ charged current) events at the energies of interest. As outgoing neutrino cannot be detected in the detector and the energy carries away by neutrino in a neutral current event which will look like lower energy electron neutrino charge current event. Reducing the high energy neutral current (NC) events also helps in the improving the purity of muon neutrino charge current events, basically by reducing the charged pions background. Also, high energy NC events which appear in our detector as low energy $\nu_{e} \mathrm{CC}$ events mimic our signal $\nu_{e}$ events. So cutting down the NC events overall increases the signal over background ratio.

The NuMI beam (subsec: 3.1.2) can be run either neutrino or anti-neutrino mode with switching the horn polarity. When the horn polarity is in neutrino mode the wrong sign component is $1.8 \%$ in the near detector in the range of $1-3 \mathrm{GeV}$ neutrino energy. The background $\nu_{e}+\bar{\nu}_{e}$ is about to $0.7 \%$ and the rest are the $\nu_{\mu}$ which is all about $97.5 \%$ in the $1-3 \mathrm{GeV}$ region as shown in right Fig 3.4 . 

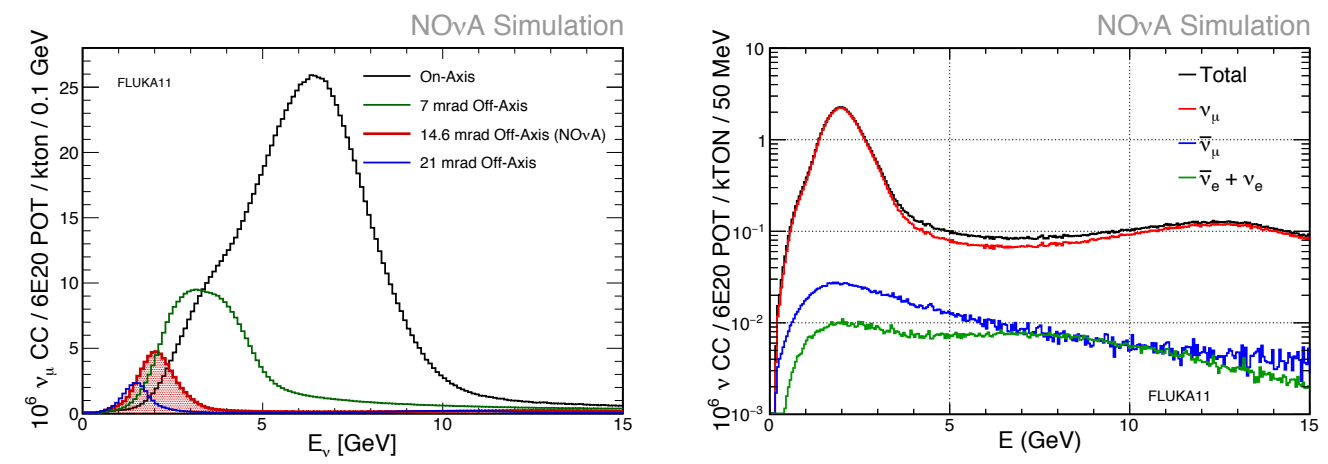

Figure 3.4: The neutrino energy spectra for near detector for various off-axis angles and the y-axis represents expected number of charged current $\nu_{\mu}$ events (left) and the flux times cross-section for different neutrino components in the NuMI beam as a function of neutrino energy (right).

\subsection{The NOvA Detectors}

In the NOvA long-baseline neutrino oscillation experiment, the muon-neutrino beam from the NuMI passes through two functionally identical detectors. One of them located at Fermilab, the other one at $810 \mathrm{~km}$ away Ash River, MN. The two detectors are placed $14.6 \mathrm{mrad}$ off-axis from the center of the neutrino beam and their similarity in their physical structure and architecture of the data acquisition help in reducing the impact of systematic uncertainties.

\subsubsection{The Cell}

Both detectors are made of reflective PVC tubes (cells) (Fig.3.7, right) that is filled with liquid scintillator. Each cell contains one fiber, corresponding to one pixel of the detector and 32 cells make up one module (Fig. 3.6). The cells of 16 make a PVC extrusion (Fig. 3.5) and two of 16 cells are glued together to form a module. Again a series of modules glued together form a plane ( 1 plane $=12$ modules in Far Detector and 3 modules in Near Detector. The cells are $3.6 \mathrm{~cm}$ by $5.6 \mathrm{~cm}$ in cross-section and $4.2 \mathrm{~m}$ in length in the near detector (ND) and $15.2 \mathrm{~m}$ long in the far detector (FD). In order to provide three-dimensional tracking of particles, a special arrangement of stacking of cells are made in alternating horizontal and vertical planes. Titanium dioxide is coated inside each cell, which helps as a reflective agent to improve the quantity of light collection in the scintillator. A wavelength-shifting fiber is looped through inside the cell and collects light from the energy deposition of a charged particle with the liquid scintillator. The fiber ends terminate on a single pixel of an avalanche photodiode (APD) (Fig.3.9).

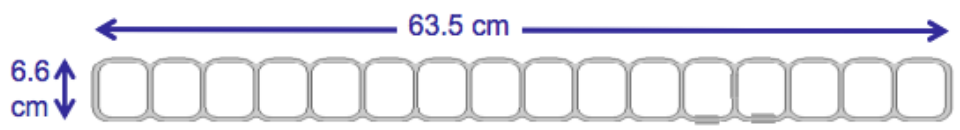

Figure 3.5: A photo of PVC cell extrusion 


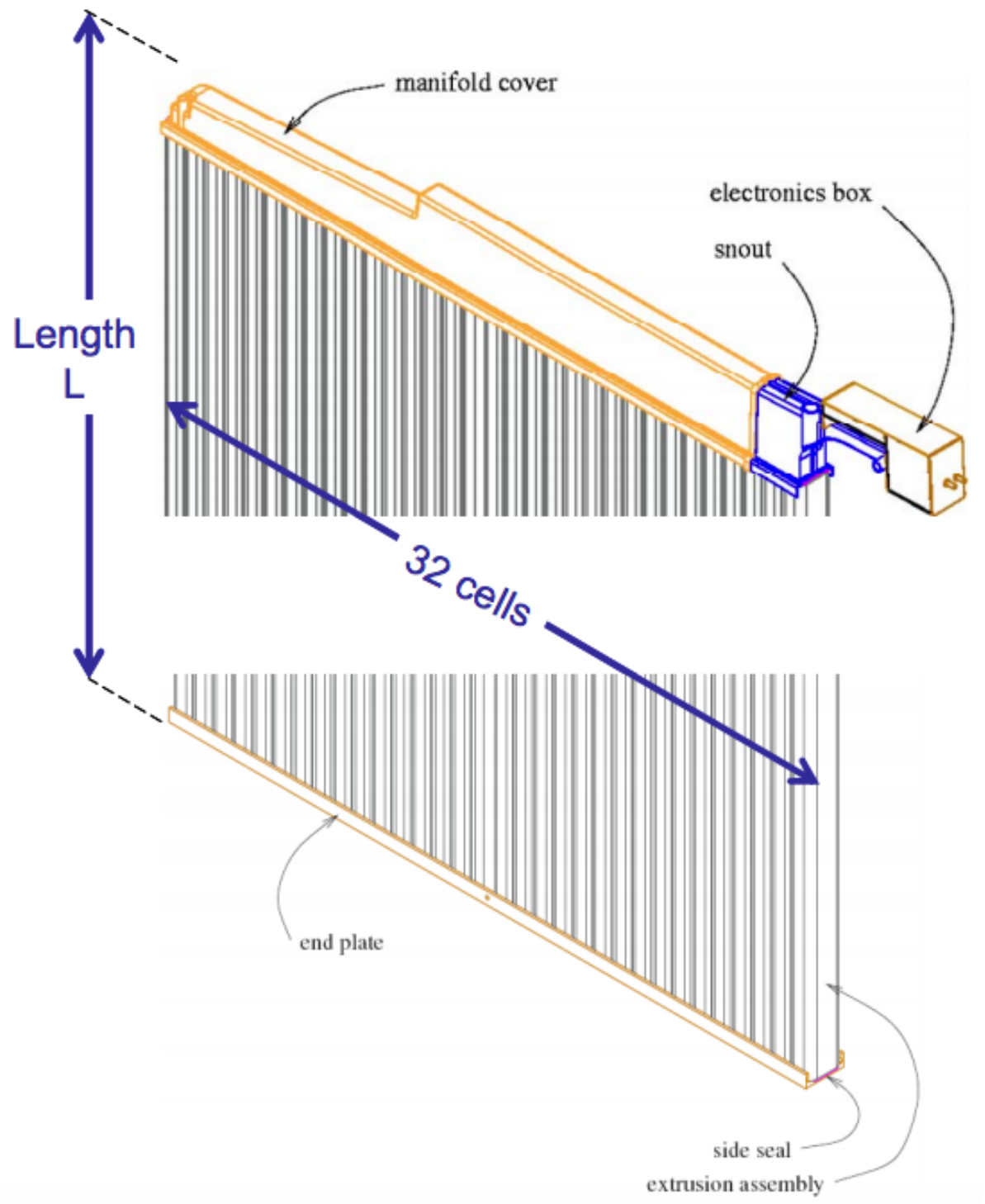

Figure 3.6: A schematic view of module and plane is formed by connecting modules side by side. 


\subsubsection{The Liquid Scintillator}

The liquid scintillator solution on NOvA consists of several scintillating agents. There is $5 \%$ of pseudocumene (1,2,4-trimethylbenzene) to emits light in the range of $270-320 \mathrm{~nm}$ region and $95 \%$ of mineral oil used as the solvent. It also contains two wavelength shifters, PPO $(2,5-$ diphenyloxazole) that excites photons of wavelength in the range of $340-380 \mathrm{~nm}$ and bis-MSB (1, 4-bis-(2-methylstyryl)-benzene) de-excites photons in the range of $390-440 \mathrm{~nm}$. Also, stadis-425 is used as an anti-static agent to make less fire hazard from charge build up and sparking during filling. Furthermore, an anti-oxidant (tocopherol (Vitamin E)) is used to avert yellowing of the scintillator, which would break down its transparency over time. The summary of the composition of the liquid scintillator is shown in Table 3.1.

\begin{tabular}{lll}
\hline Component & Purpose & Mass Fraction (\%) \\
\hline Mineral Oil & Solvent & 95.8 \\
Pseudocumene & Scintillant & 4.1 \\
PPO & Wave shifter & 0.091 \\
bis-MSB & Wave shifter & 0.0013 \\
Stadis-425 & Anti-static agent & 0.0003 \\
Vitamin E & Antioxidant & 0.0010 \\
\hline Total & & 100 \\
\hline
\end{tabular}

Table 3.1: Composition of liquid scintillator [66].

\subsubsection{The APD}

The APDs are a critical component of the experiment, as they convert the photons produced by charged particles traversing the scintillator in the PVC tubes of the detectors into electronic signals. The electronic signal is amplified and read out by a Front End Board (FEB) of which there is one for each APD. The APDs used by NOvA detector is manufactured by Hamamatsu. The working functionality of APD is as follows: Firstly the electron-hole pairs excited by photons are accelerated with a strong electric field under the condition of high reverse-biased voltage. Due to impactionization process (where the acceleration of highly energetic electrons strike other electrons and makes them discharge and again these secondary electrons and holes are themselves accelerated and strike more electrons makes them free and so on) an avalanche of charge carriers in the diode leads to a significant amplification of the photo-current.

The quantum efficiency of APD is $85 \%$ for the wavelength of the light signal in the range of $520-550 \mathrm{~nm}$ and it also helps in observation of very low-intensity light signal in our long PVC tube. To minimize the thermal noise, the current generated in the absence of light (dark current), each APD is cooled at a temperature of -15 degree Celsius through a thermoelectric cooler (TEC). The excess heat is removed by continuously flowing the cool water also to minimize the surface currents, we need to make clean and dry on the surface of APD by coating with Paralene. Dry nitrogen gas flows through a system of tubes to the APD housings to keep ice from forming on the surface. Each APDs are operated at a voltage of $425 \mathrm{~V}$ to produce a gain of about a 100 . The signal is amplified by integrated circuits. The FEB digitizes the hits above the threshold and reads the analog signal from the APD. The hit information from the FEB is collected by a Data Concentrator Module (DCM). 
DCMs passes the data to a processing farm.

\subsubsection{Far Detector}

In total there are 896 planes with 344,064 channels in the far detector with a total of 14 kt active detector mass of which $65 \%$ is liquid scintillator. There are two sets of 32 plane blocks glued together to form 14 di-blocks for the far detector. Each di-block has 12 DCMs, with 64 FEBs in each DCM. The FD is on the surface and receives $50-70$ cosmic rays in each 550 microsecond readout window. Its primary goal to measure the energy spectra for our beam neutrinos, separating muon and electron neutrino charged-current interactions from neutral-current interactions.
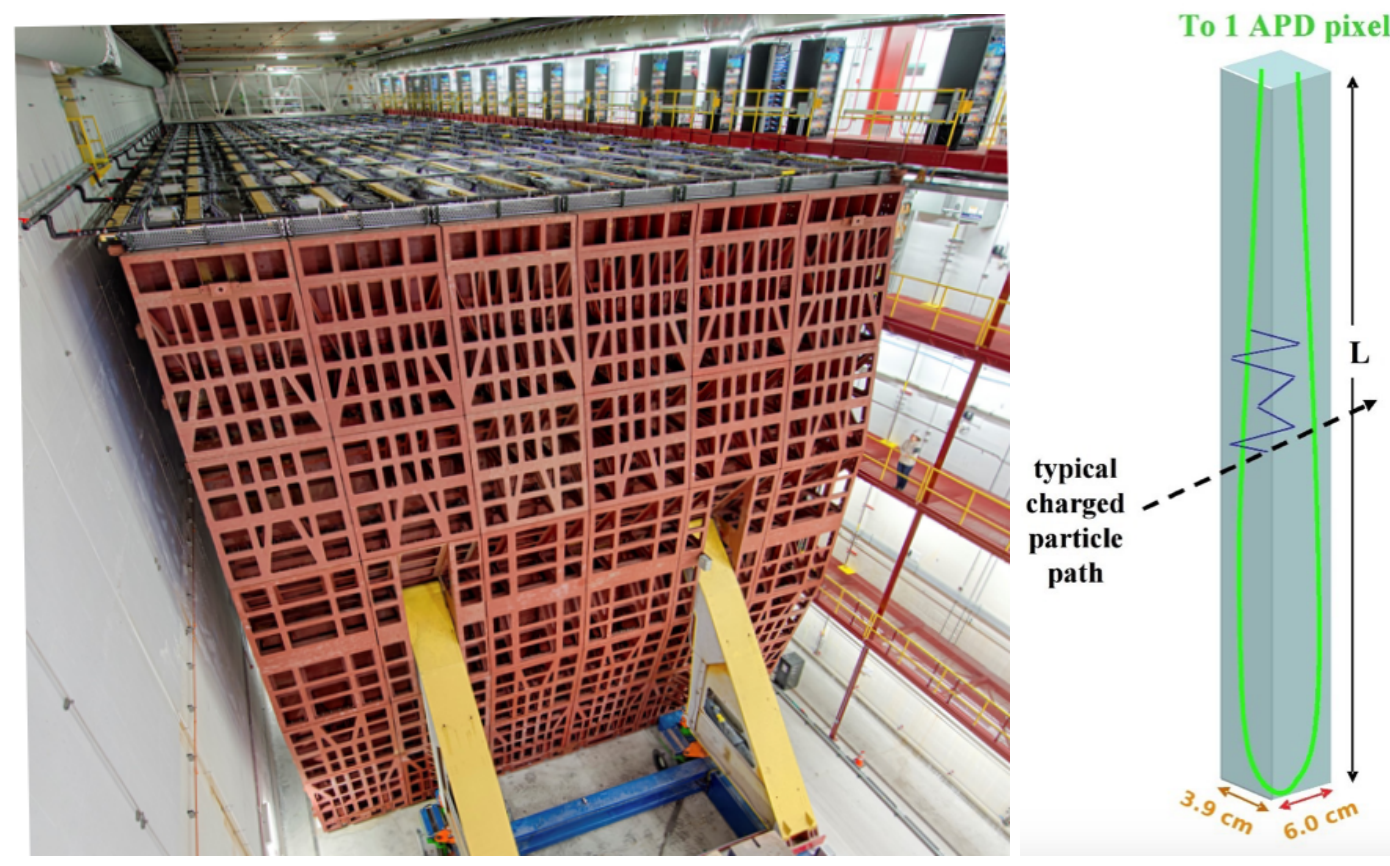

Figure 3.7: Photograph of the NOvA far detector (left). Schematic diagram of NOvA cell, the walls are made up of PVC with a loop of wavelength shifting fiber is read out by one APD (right).

\subsubsection{Near Detector}

The ND is $105 \mathrm{~m}$ underground to reduce cosmic ray interactions also to catch the NuMI beam as the beam is running downwards at an angle of 58 milliard. It is only $1 \mathrm{~km}$ away from the beam, so the neutrino interaction rate is much higher than the far detector. It is designed to measure the unoscillated beam spectra to predict the far detector spectra. The length of the detector is 15.9 meters long along the beam direction. As shown in Fig.3.8 (right), it is further divided into a two region, one is 12.67 meter active region followed by a 3.23 meter-long muon catcher at the downstream end. The muon catcher consists of alternating steel planes and scintillating planes to increases the efficiency to contain muons. The ND totals 290 tons, 130 tons of which is liquid scintillator. The active region has a mass of 193 tons with $\sim 18 \mathrm{k}$ channels and 192 planes followed by 22 planes and 10 steel planes which are $10 \mathrm{~cm}$ thick. The dimension of the active region is $3.9 \times 3.9 \times 12.67$ 
$(h \times w \times l)$ meters. The active region electronics are instrumented in 3 di-blocks. The di-block consists of two DCMs each for the vertical planes and horizontal planes. Furthermore, some DCMs are occupied with 64 FEBs and the other at 32. The muon catcher consists of a periodic horizontal plane and vertical planes of steel and liquid scintillator. In the muon catcher, there are two DCMs, one for the horizontal and another one for the vertical module.
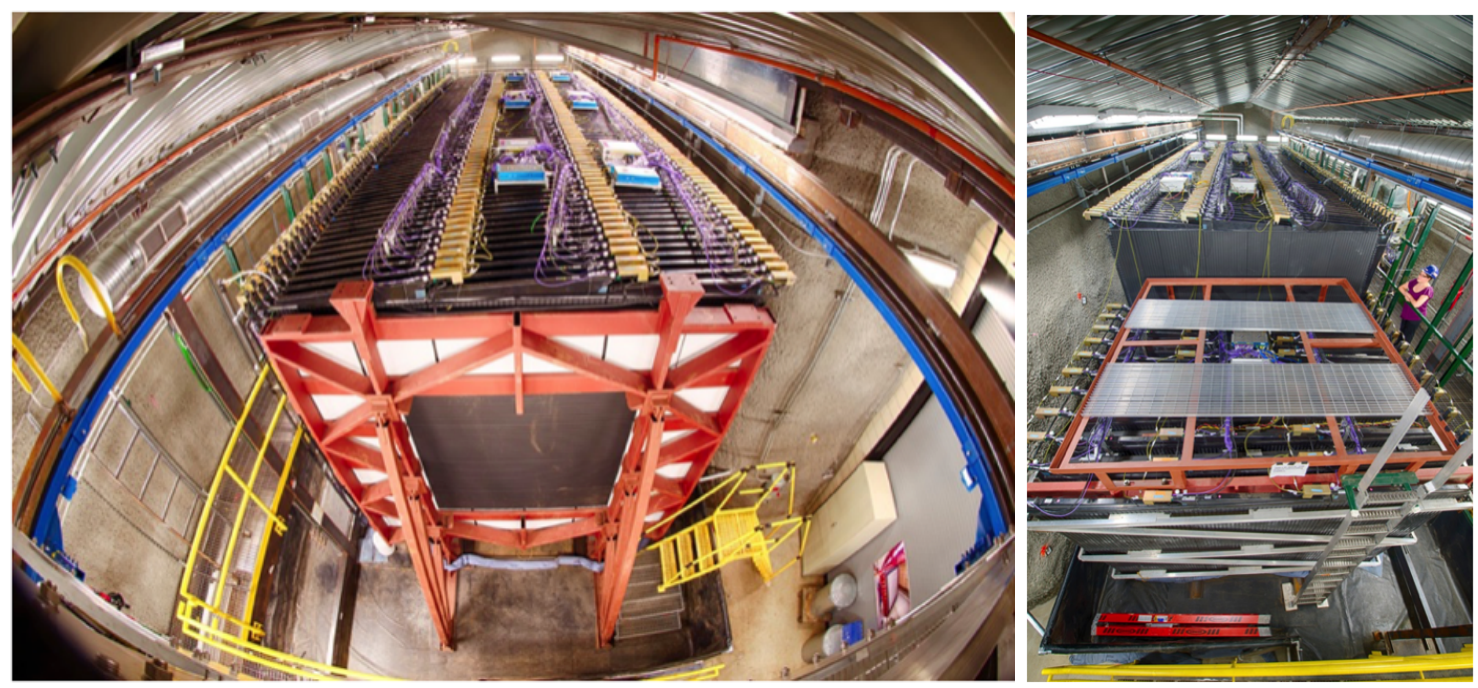

Figure 3.8: NOvA near detector (left) and muon catcher region (right) with steel plates alternating with scintillation planes, whose height is two-thirds that of the active region.
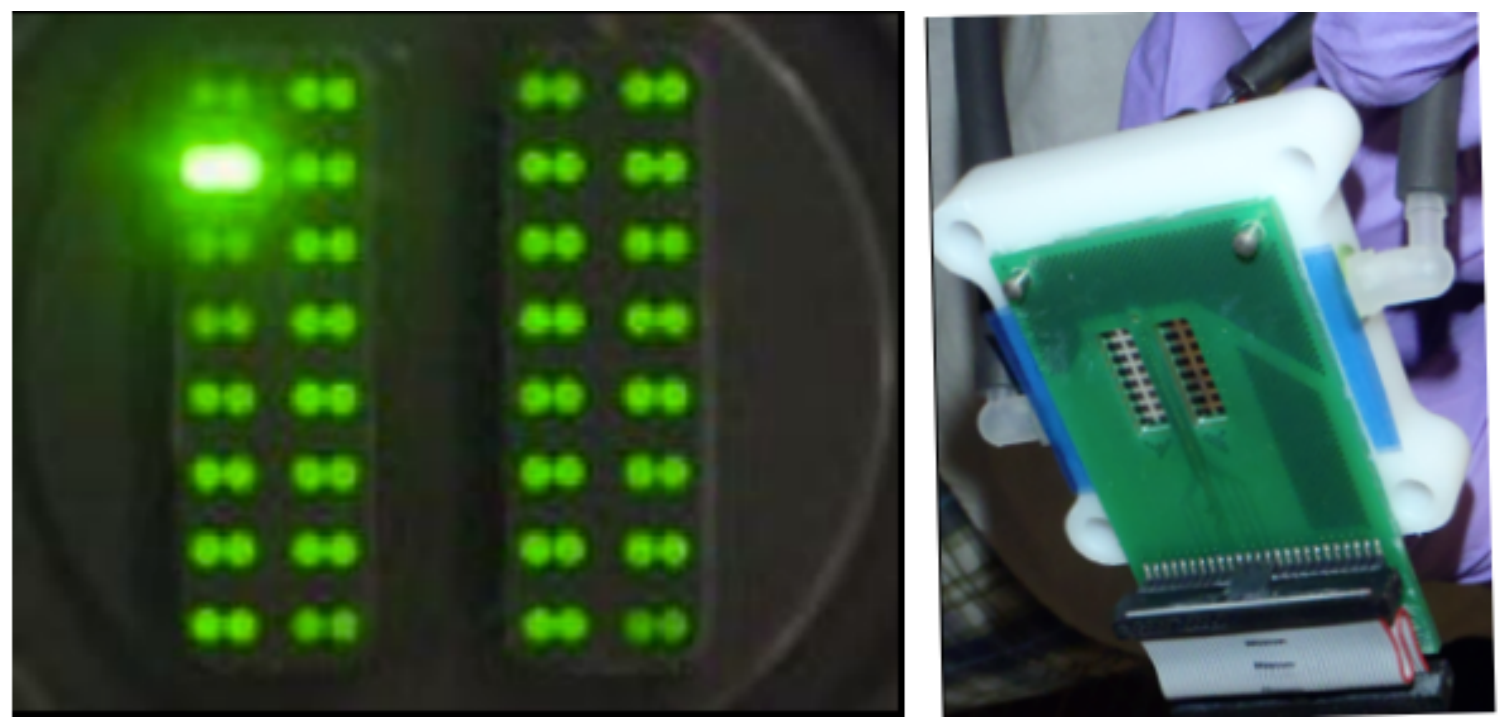

Figure 3.9: The ends of 32 wavelength-shifting fibers collected at the end of scintillation cells to mount to an APD (left). The front face of an APD will be pressed against the fiber ends (right). 


\subsection{Overview of Data Acquisition System}

The photon signal from 32 individual cells is amplified by APD. Each APD is connected to a single Front End Board (FEB) which has four circuits. The raw hits voltage signal arrived from APD is transmitted to an application specific integrated circuit (ASIC) which shapes the voltage signal so that a hit can be read out on a time scale. The output from ASIC is propagated to an (analogto-digital converter) ADC to digitize the signal and also tags the signal hits which are above the threshold. The hits which pass threshold are redirected towards a field programmable gate array (FPGA) where they are time-stamped for each hit in the channel. In each channel, FPGA also programmed for rising and fall-time in the ASIC and threshold in ADC. Another component of FEBs is thermoelectric cooler (TEC) which measures the APD temperature and maintained at -15 degree $\mathrm{C}$ to get a stable gain.

Each FEB digitizes the hits from 32 individual channels or pixels. A Data Concentrator Module (DCM) is connected to a maximum of 64 FEBs. In the FD all 168 DCMs are connected to the maximum number, whereas in the ND the 14 DCM's are connected to between 22 and 64 FEBs. The DCM uses custom software to collect continuously all the data of nanoslices and combines them into a $50 \mu s$ slices called microslices [68]. FPGA in DCM pools the FEBs to collect nanoslices and if there is missing header information, we call them a corrupt microslice. When building a microslice, DCM does checks on it, and if any fail, a microslice is flagged as corrupt. Corruption can be caused by a bit being flipped, or a misbehaving FEBs, etc. These are pretty transient and the exact cause isn't always known. If a DCM continuously spits out a lot of corrupt microslices, this is an indication of hardware failure and it is usually swapped out. The schematic view of DAQ data flow is shown in Fig 3.10.

DCMs send microslices to Buffer nodes, and it follows a round robin system. All DCMs send their first microslice to first buffer nodes, and the second microslice goes to the second Buffer node. DCMs have a simple algorithm to decide which buffer node the microslice should be sent to. The Buffer node opens a millislice, get microslices from each DCM. When a millislice is complete, it is copied to the shared memory, where the trigger messages from Global Trigger read it out. Each buffer node keeps a long pool, $\sim 8 \mathrm{~GB}$ worth of data. When a microslice is read out, it is not deleted from the node, as new microslices come in, the old ones expire and delete themselves. A Buffer node that does not have a microslices matching a trigger, it will send an empty data block (set of all triggered microblocks (set of all DCMs microslices) for an event). All data for a triggered event

goes to one and only Data logger. Data logger waits until it hears from every buffer node before writing a trigger event to the data disk which is capable of saving an event to multiple streams.

The far detector receives a high rate of cosmic events roughly $100 \mathrm{kHz}$ as it is on the surface and low rate (a couple of events per day) of beam neutrino events. The NuMI beam spill trigger receives timestamp sent from the Fermilab accelerator with a time correction for the neutrinos time of flight. When NuMI beam spill received by the detector, the buffer farm selects 1000 microblocks or $500 \mu \mathrm{s}$ slices of data for the entire detector and writes to the data disk. The beam data is collected within $10 \mu s$ beam spill in the range of $218-228 \mu s$. A trigger has a start time and an end time and a trigger ID. The detector also writes data from other triggers for exotic search and these triggers are out of focus in this thesis.

The DAQ system in the NOvA detector are synchronized through the timing system and more can be found in [69]. Timing Distribution Units (TDU) is like a DCM. It sends out timing commands 


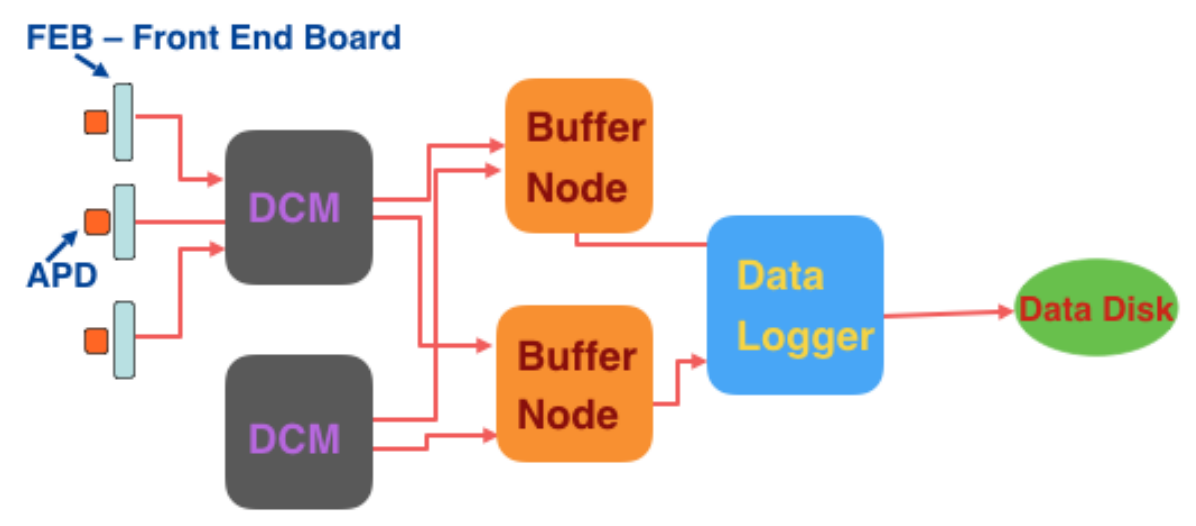

Figure 3.10: Schematic drawing of DAQ data flow

to all DCMs. When a DCM receives it, it distributes it to all the FEBs. FEBs have the same cable length from the DCM. The Master Timing Distribution Unit (MTDU) extracts the timing info from universal time based on a link to the global positioning system (GPS) and distributes the timing signal to the first Slave Timing Distribution Units (STDUs) in the chain. Each channel in the NOvA is synchronized in this manner and with each hit in the detector, corresponding time stamp are registered. This MTDU system at Fermilab is also used to decode time stamp into NOvA time for the beam spill information coming from the NuMI accelerator and the corrected spill time transmitted further into the near and far detector.

\subsection{Performance of the NOvA Detectors}

For every particle physics experiment, the success depends on the monitoring of the detector performance. The NOvA experiment has various tools developed to track the detector performances. The high quality of data is monitored by performance metrics developed through the automated script. One metric that is used to gauge the overall performance of the detectors is their fractional up-time, as shown in Fig.3.11. Maximizing uptime is a top priority for the experiment because while one may have any number of sophisticated tools for reconstructing neutrino kinematics, they are of little use if the data was never recorded in the first place.

\subsection{Exposure}

Exposure is important to scale the distributions of selected events. In NOvA experiment useful exposure are livetime, the mass of the detector and protons on-target (POT). The cosmic rays and electronic noise is scaled using livetime, however for accurate normalization if some part is blocked in the detector, active mass of the detector is necessary. The production of neutrino is proportional to the number of pions decayed, further it depends on the number of protons delivered to the graphite target. The exposure of neutrinos is tracked spill-by-spill and stored in a database. The total number of neutrino exposure for neutrino and anti-neutrino mode as a function of time since NOvA began to run is shown in Fig. 3.12. The accumulated POT for this analysis is $8.09 \times 10^{20}$ POT of neutrino beam collected between August 2014 and February 2017. 


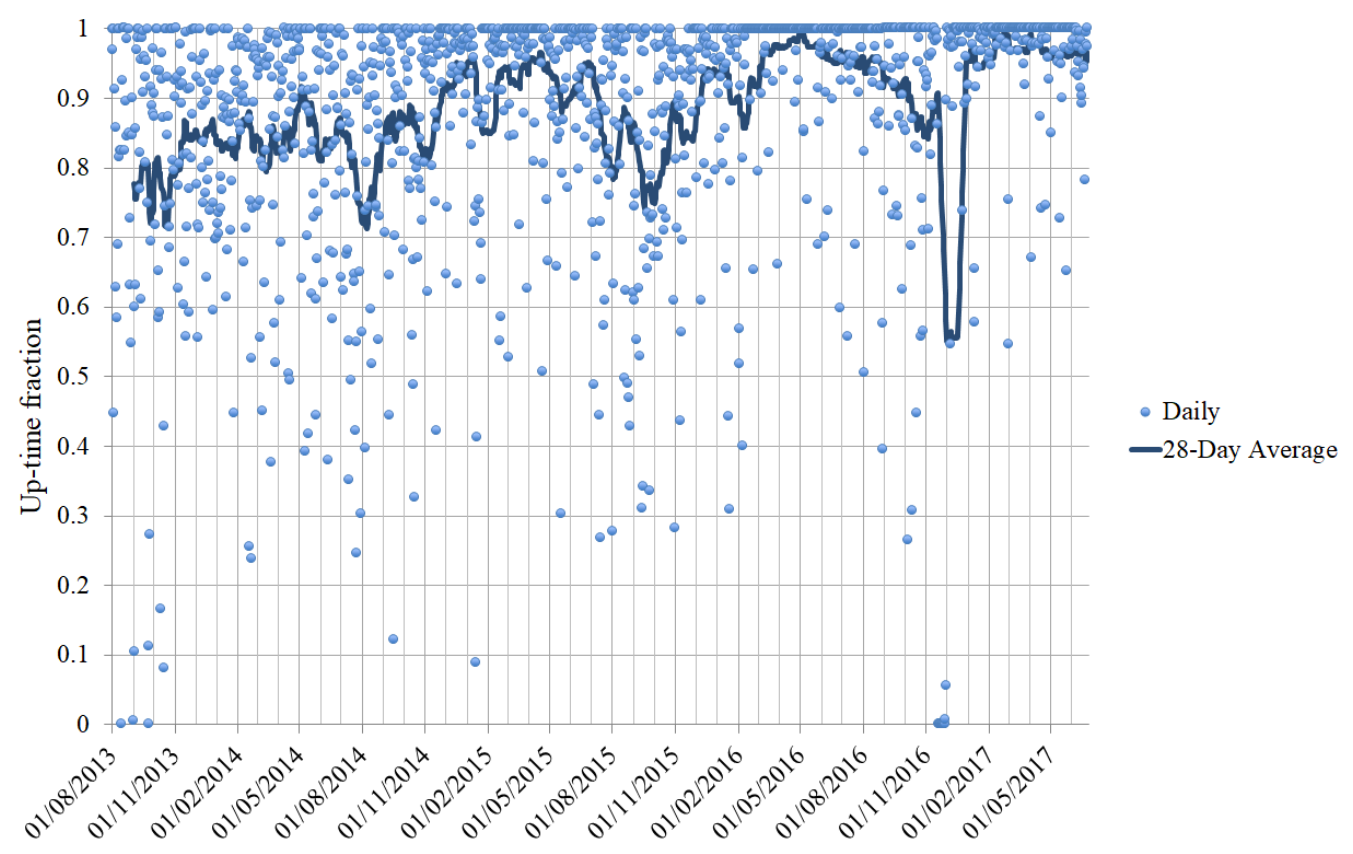

Figure 3.11: Up-time fraction as a function of time. Uptime has steadily increased over time as the experiment moved from commissioning to steady state running.

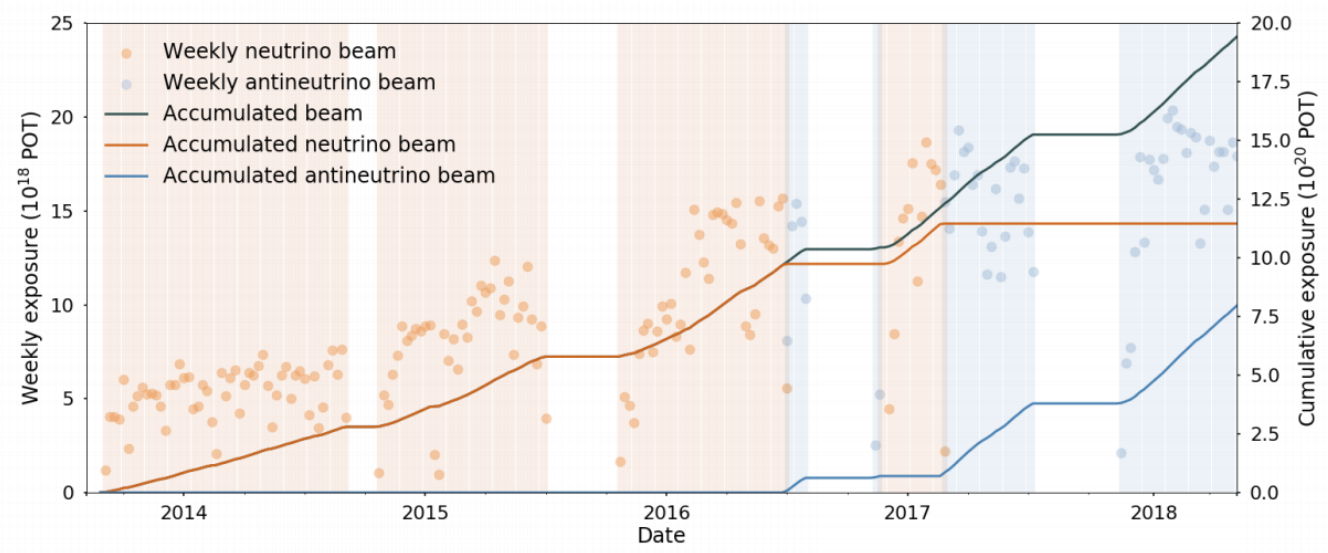

Figure 3.12: Weekly exposure and cumulative exposure vs date from starting of NOvA data taking in neutrino mode as well as anti-neutrino mode. 


\section{Chapter 4}

\section{Simulation}

Simulation plays a significant role in each and every experiment we do perform. The simulation helps in several steps in analyzing data, such as to make a measurement, an independent set of data to train particle identification and tune to our analysis, determining the selection efficiency, to estimate the neutrino energy and the background components associated to signals. The simulation chain for the NOvA experiment consists of two main part, one is beam simulation and another is detector simulation. This chapter discusses all piece of the NOvA simulation chain starting from neutrino production to readout.

\subsection{Flux Prediction}

NuMI beam simulation starts from the outset by simulating the interaction of $120 \mathrm{GeV}$ protons from Main Injector with graphite target and ends with the creation of flux files which contains flavor types, directions, energy and momentum of the simulated neutrino. Flux expected from the NuMI beam is simulated using G4NuMI, which is a GEANT4 [70] based Monte Carlo simulation. G4NuMI simulates hadron production upon interaction of the incoming protons with the NuMI target, further which traces the decay of neutrinos or absorption of muons and hadrons by the hadron monitors at the downstream region. Neutrinos produced from decay products of particles are saved for further use in the simulation. Simulating all the final products of particles interact with the target is time-consuming as well as expensive on computing resources, so every particle is not tracked. The kinematic properties of the neutrino parents in flux files are saved in addition to neutrino flavors, directions, decay point, momentum, and energy. This information can later be used and reused to generate neutrinos to interact in the NOvA detectors. Also keeping parent information helps in the event re-weighting based on hadronic model studies.

\subsection{Neutrino Interactions}

Beam neutrino interactions in NOvA detectors are modeled by GENIE [71] (version 2.12.2) event generator which takes input as flux file. GENIE model creates interactions in the detector volumes using neutrino created from the beam simulation and the neutrino interaction cross-sections to determine the energy of neutrino which interacts. GENIE also simulates the different types of 
neutrino interaction (QE, RES, DIS, COH and MEC (details in chapter 2)) using various crosssection models. The outcome of this model is the list of final state particles that originate from the interaction of the nucleus of the detector material and kinematics are saved for each particle.

The quasi-elastic scattering is modeled on the basis of Llewellyn-Smith formalism as described in section 2.5.1. GENIE uses a dipole form with the axial vector mass $M_{A}$ of a default value is $0.99 \mathrm{GeV} / \mathrm{c}^{2}$ and the electromagnetic form factor is parameterized using the BBBA2005 model [72]. The Rein-Sehgal model [73] is used to simulate the resonance neutrino-nucleus interaction process and the default value for the resonance axial vector mass $M_{A}$ is $1.12 \mathrm{GeV} / \mathrm{c}^{2}$. Genie uses 16 baryon resonances in its model out of 18 resonances from its original paper. Similarly, the coherent scattering process is modeled using Rein-Sehgal model [73] where the events lie within the low $Q^{2}$ region, where expansion around the PCAC hypothesis predicts a single dipole form factor. The deep inelastic scattering (DIS) interaction uses Bodek and Yang model at low $Q^{2}$ value. Several cascade hadronization models are available based on various experimental measurements and theoretical models to determine the final state particles and their kinematics. The custom AGKY model [71] is the default hadronization model in the GENIE. The events which are represented by low invariant mass are modeled using Koba-Nielsen-Olesen (KNO) scaling [74], however, the PYTHIA/JETSET model [71] is used for higher invariant masses. GENIE uses an empirical MEC model to explain discrepancies seen with data near $1 \mathrm{GeV}$ in the $Q^{2}$ region between $\mathrm{QE}$ and resonant events.

The final state interaction (FSI) rates are obtained from the free hadron cross-sections and the density of nucleons. GENIE uses Intranuke to simulate final states interactions. Due to the difficulty to make a measurement, there are large uncertainties on these models which directly impact the observed energy of neutrinos in the experiments. In addition to cross-section prediction, GENIE also provides systematic uncertainty on various parameters involved in the cross-section models according to global fits to make error bands on the prediction of various analysis.

We also simulate detector volume as well as surrounding materials in the detector hall. In the near detector, neutrino interactions occur in the rock upstream of the detector which is outside of the detector hall that results in particle interactions (outcome from neutrino - rock interaction) in the detector at the same time as neutrinos that interact within the detector. Rock interactions are simulated separately and independently from the in-detector events due to the complexity of simulating them and the fact they are computationally expensive to simulate. Simulated rock events are smaller in total number, later it is overlaid randomly with the detector events so that the total number of neutrino interactions in the detector is consistent with what is expected. Later, in analysis cut stage, rock interactions are rejected. So this has a negligible impact on the results of the analysis.

\subsection{Propagation of Particles}

The output from GENIE [71] is fed into GEANT4 [70] for particle propagation through detector geometry. The geometry includes the NOvA detectors, detector halls, and the surrounding rocks are encoded in CERNs Geometry Description Markup Language (GDML) files which are further parsed by ROOTs geometry classes. GEANT4 simulates particle propagation and saves the energy depositions until the particles have less than $100 \mathrm{eV}$ of kinetic energy remaining. GEANT4 can not offer a single modeling algorithm, especially for hadrons to cover the entire energy range from zero to $\mathrm{TeV}$, for all known processes and particles. The physical processes are modeled via different 
physics lists. $Q G S P_{-} B E R T_{-} H P$ is our standard physics list applying the electromagnetic processes, Bertini-style cascade (BERT) modeled up to $9.5 \mathrm{GeV}$, Quark Gluon String (QGSP) model for high energies $(\sim 20 \mathrm{GeV})$ interactions of protons, neutrons, pions, kaons, and nuclei. The high energy interactions create an excited nucleus, which is passed to the pre-compound model for the nuclear deexcitation. In addition to that, it uses the data-driven high precision (HP) neutron model used to transport neutrons below $20 \mathrm{MeV}$ down to thermal scattering energy. The output from GEANT4 is the individual particle energy depositions which are most important for the next step of simulations.

\subsection{Transport of Photon in Detector}

GEANT4 determines how much energy is deposited by particles in the NOvA detector cells. The conversion of scintillation light to electrons, that are read out from each channel or cells. So greater charged produced in the APD, more scintillation light produced hence high energy deposited in the cell. The scintillation light which will scatter, absorbed and transported via the wavelength shifting fiber and the signal will transmit to the APD. The captured signal is simulated from custom NOvA software developed internally by NOvA analyzers.

A ray-tracing algorithm has been developed to compute the collection of scintillation photons by the fiber (Fig. 4.1). Following assumptions are made for the parameters of the detector, such as 9 ns scintillator emission time, uniform cell wall reflectivity of $87.7 \%$ (Fig. 4.1), 1.47 as the index of reflectivity in oil, and an exponential photon capture probability with a capture length of 30.66 $\mathrm{cm}$. As mentioned earlier in chapter 3, the NOvA detectors are composed of many identical readout cells and all are treated identically. A template function is generated which was determined from a ray tracing simulation to parameterize photon transport.

Transportation of photons through fiber determines how many photons are expected to arrive at the APD as a function of time given that the photons are collected in the fiber. All the captured photons are split, with half traveling in each direction along the fiber, and attenuated according to measured results from fiber quality control tests. Finally, the number of photo-electrons absorbed by the APD is determined after considering the quantum efficiency and Poisson statistics. In addition, APDs have an excess noise factor that expands the variance of the detected signal besides to the Poisson variation induced by the number of photo-electrons captured by the APD. It is modeled with the theoretical distribution as a log-normal distribution.

\subsection{Readout Simulation}

The next stage of detector response is the simulation of the front end board (FEB) electronics. FEB contains three chips, an application-specific integrated circuit (ASIC), an analog-to-digital converter (ADC), and a field programmable gate array (FPGA). ASIC pre-amplifies and pulse shaping the photoelectrons signal. Preamplifier integrates the charge produced by the APD and a pulse is generated from capacitor resistor differentiator and resistor-capacitor integrator (CR-RC) circuit with a fast rise time controlled by an integrator unit and a slow fall time controlled by a differentiator unit. The pulse shaping was modeled using the analytical solution of the response of 

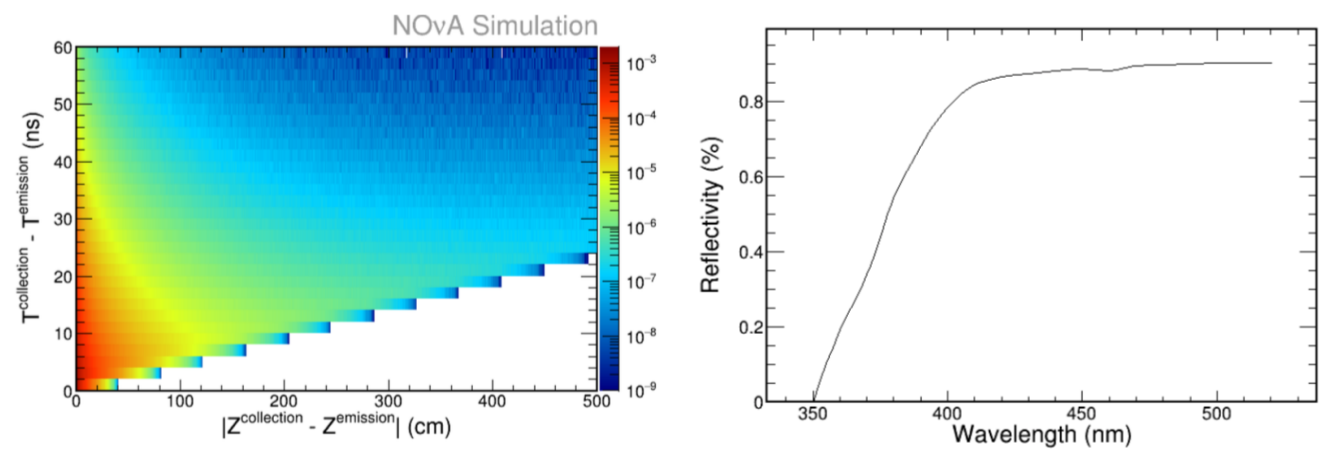

Figure 4.1: Template function of collection rate of scintillation photons (left).Cell wall reflectivity as a function of wavelength (right).

the CR-RC circuit to a unit charge impulse as

$$
f(t)=\frac{F}{F-R}\left(e^{-\frac{\left(t-t_{0}\right)}{F}}-e^{-\frac{\left(t-t_{0}\right)}{R}}\right)
$$

where $t_{0}$ is the time that the photoelectron pulse was collected by the APD, F and R is the fall and rise time of the CR-RC circuit respectively. The shaping of a pulse is input to the ADC. When photo-electrons are created by a cell, an analog traces (Eq. 4.1) are created from each of the photoelectrons individually and added together resulting in the final trace. The ADC chip digitizes 4 (8) analog traces in every $62.5 \mathrm{~ns}$ for FD (ND) by truncating the trace to integer values, and the values clamped between 0 and $4095 \mathrm{ADC}$ counts. Once the analog traces are digitized, an offset is made by a constant baseline. The value of the baseline determines the dynamic range of the output before saturation. In MC, the saturation peak has a spike (due to a constant baseline for all channels), however, in data, it was broad and occurs at lower energies. The Gaussian distribution of the baseline on a channel-by-channel basis are studied from Digital Scanning Oscilloscope (DSO) scan. A baseline in ADC units for each channel are randomly drawn at the start of the run from a Gaussian distribution with the mean and RMS value. Conversion of the trace from units of PE to ADC using a factor determined through charge injection studies and a baseline is drawn from the distribution of modes seen in pedestal scan. Finally, the FPGA is looking for peaks above threshold in the dual correlated sampling trace defined from $\mathrm{ADC}_{i}-\mathrm{ADC}_{i-3}$. where $\mathrm{ADC}_{i}$ is the value of the current digitization sample and $\mathrm{ADC}_{i-3}$ is the value of the digitization sample three time slices (details in sec5.1.1) earlier than the current one.

We also model noise for cells not containing physics hits with a data-driven method that draws noise hits from a template. A template is constructed by making a 3-D histogram containing the values adc 1 - adc0, adc 2 - adc0, and adc 3 - adc0 for hits in the noise slice. This histogram is then normalized as a product of livetime and number of active channels for all subruns so that it reflects the probability of recording a noise hit per channel and per unit time. 


\subsection{The APD Sag Simulation}

In an APD, all pixels share a common voltage source with the voltage difference driving the avalanche multiplication. When a large energy deposit occurs on one pixel, the pulse of all other pixels on the APD momentarily sag. This sag is shaped by the ASIC and the digital signal processing only measures the difference between samples at different times. The recovery of the sag is a real energy deposit and a digit is created at the time triggered. The value of the sag was determined to be 0.0186 times the size of the pulse on the channel with a real energy deposit. A sagged trace was computed using the total amount of light captured by all pixels of an APD exceeding 5000 ADC in any given $15 \mathrm{~ns}$ window. The final traces are computed even for pixels not containing physics hits. This models the FEB flashing behavior seen in data when high energy cosmic rays traverse the detector.

\subsection{Cherenkov Light Simulation}

When a particle travels through a medium if the speed of light is faster than the speed of light through a medium a radiation is emitted called as Cherenkov radiation [75]. The spectrum of energy per unit track length is described by the Frank-Tamm formula [76]:

$$
\frac{d^{2} N_{\gamma}}{d x d \lambda}=\frac{2 \pi \alpha z^{2}}{\lambda^{2}}\left(1-\frac{1}{\beta^{2} n^{2}(\lambda)}\right)
$$

where $\beta$ is the speed of the particle, $\mathrm{n}(\lambda)$ is the refraction index, $\lambda$ is the wavelength. The energy spectrum is changes $\sim 1 / \lambda^{2}$ if the refraction index changes slowly. At the wavelength between 400-500 nm, the scintillation light is absorbed by the wavelength shifting fiber and the Cherenkov radiations are indistinguishable from scintillation light which magnifies our light production for fast particles. The Cherenkov light production as a function of wavelength for the different velocity of particles is shown in Fig. 4.2. The refraction index of mineral oil used by NOvA is 1.47 and Cherenkov light accounts for roughly $4 \%$ of the light collected by fast particles is shown in Fig.4.2.
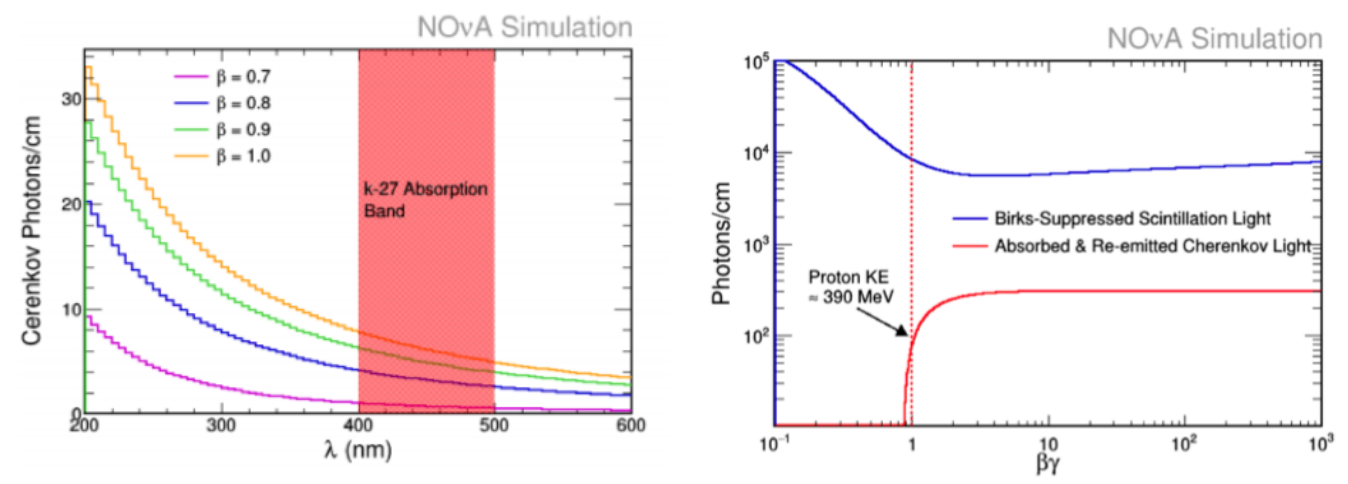

Figure 4.2: The Cherenkov light production as a function of wavelength for different velocity of particles $(\beta)$ and the red shaded region is absorption spectrum of the k-27 dye in the optical fiber(left). The photon production comparison as a function of $\beta \gamma$ for scintillation is in blue and Cherenkov radiation is in red (right). 


\subsection{Birks-Chou Parameters}

The conversion of energy deposition and light yield is determined from GEANT4. The light yield along the path of the particle is directly proportional to the energy lost along the path for lower energies. However, at high energy loss per unit length, organic scintillators undergo recombination and quenching effects which reduce the final light yield. Birks Law attempts to model this empirically using a second-order correction term [77, 78] :

$$
\frac{d L}{d x}=\frac{L_{0} \frac{d E}{d x}}{1+k_{B} \frac{d E}{d x}+k_{C}\left(\frac{d E}{d x}\right)^{2}}
$$

where $k_{B}$ and $k_{C}$ are the Birks' and Chou constants and these constants are determined by selecting ND events with single muon and single proton tracks. Comparing the $\mathrm{dE} / \mathrm{dx}$ distributions as a function of a number of planes from the end of the track in data and MC. The values producing the best data/MC match were $k_{B}=0.040 \mathrm{gMeV}^{-1} \mathrm{~cm}^{-2}$ and $k_{C}=-0.0005 \mathrm{MeV}^{-2} \mathrm{~cm}^{-2}$. With this large of a Birks suppression, the energy in the last plane would be over-suppressed; the negative contribution from the Chou constant remedies this over-quenching. There is a tunable parameter in the conversion factor between energy deposited in the detector and scintillation photons created. In NOvA scintillator we expect 3360 photons/MeV. Light levels were tuned using only the $3 / 4$ of the cell to avoid roll-off regions while tuning to the dimmer regions of the cell, the scaling of $\mathrm{MC}$ attenuation curves is obtained in both $\mathrm{X}$ and $\mathrm{Y}$ view from a good match between data/MC. For ND X and Y, view factors are 0.610058 and 0.598244 respectively. The data/MC comparison for detector response to cosmic ray muons without offline calibrations for ND is shown in Fig. 4.3.

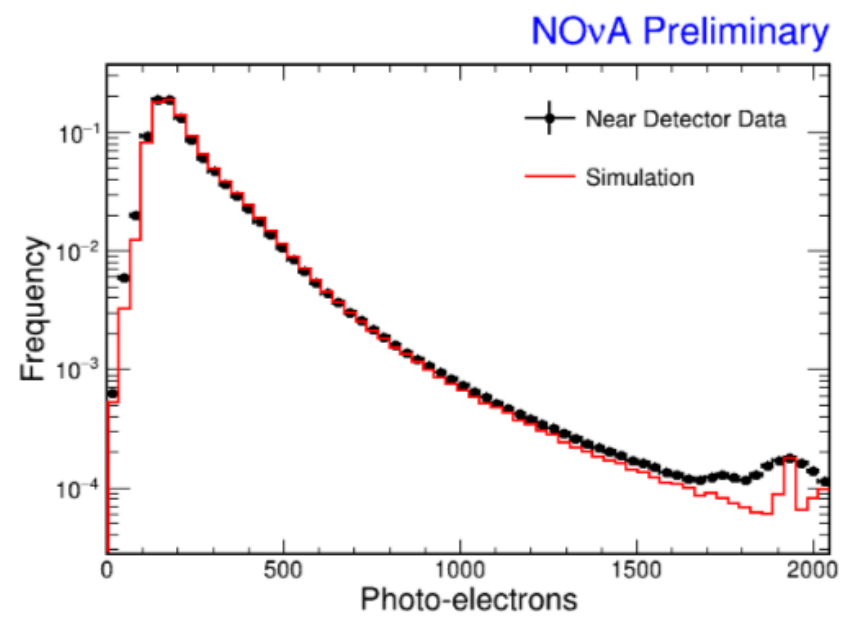

Figure 4.3: A comparison of the photo-electron spectrum in cosmic rays at the ND in data and simulation. The discrepancy between data and simulation at high photo-electrons results from the gain being set on each cell individually in the detector while the simulation models that gain as a single value across all cells. 


\section{Chapter 5}

\section{Reconstruction and Calibration}

\subsection{Reconstruction}

The analysis goals of the NOvA experiment require detailed reconstruction of the neutrino interactions. The signature of events (individual fundamental interactions) looks different for different interactions. The $\nu_{e}$ charged current interaction produces an electromagnetic electron shower. Whereas $\nu_{\mu}$ charged current interactions produce a muon as a narrow track along its trajectory rather than a shower (Fig.5.1). Apparently single $\pi^{0}$ creates more difficulty in neutral current interactions. The electromagnetic showers produced by the two photons decay of $\pi^{0}$ appear to be troublesome to separate from electrons. The scintillating light is produced hence photon converts into an $e^{-} / e^{+}$pair but prior to that, it travels some distance (the photon conversion distance is $\sim 38 \mathrm{~cm}$ (6 planes)). Therefore, we have developed different kinds of reconstruction tools in the NOvA experiment for different purposes. The full chain of reconstruction is outlined in the following sections. The NOvA detectors collect raw data from the readout, referred to as cell hits (activity on a particular cell), it saves information about the plane, cell, time and charge information about the hits. Spatial and temporal correlation between the hits is clustered together into different groups called "slices" which act as the basis for reconstruction at a later stage is explained in section 5.1.1. Next, straight line features in slices that serve as seeds are identified by modified Hough transformation (Section 5.1.2). Then, the Hough lines are used to reconstruct a global 3D neutrino interaction vertex using an Elastic Arms algorithm (Section 5.1.3). Prongs (considered as a collection cell hits having start point and direction) are produced by "fuzzy-k-means" algorithm and posses the activity of particles in the event, using vertex as a seed (Section 5.1.4). Another tracking algorithm, based on a Kalman filter generates reconstructed tracks from individual slices and the goal of the tracking is to trace the trajectory of individual particles that deposit energy in the detector. This is especially useful in identifying particles that do not create large electromagnetic or hadronic showers, such as muons (Section 5.1.5).

\subsubsection{Isolating Neutrino Interactions}

NOvA collects data in the form of packets of hits in 550-microsecond readout window for the entire detector. However, physics interactions take place within a shorter time period. In the FD which is located on the surface, the primary concern is separating 50-70 cosmic rays in 550-microsecond 


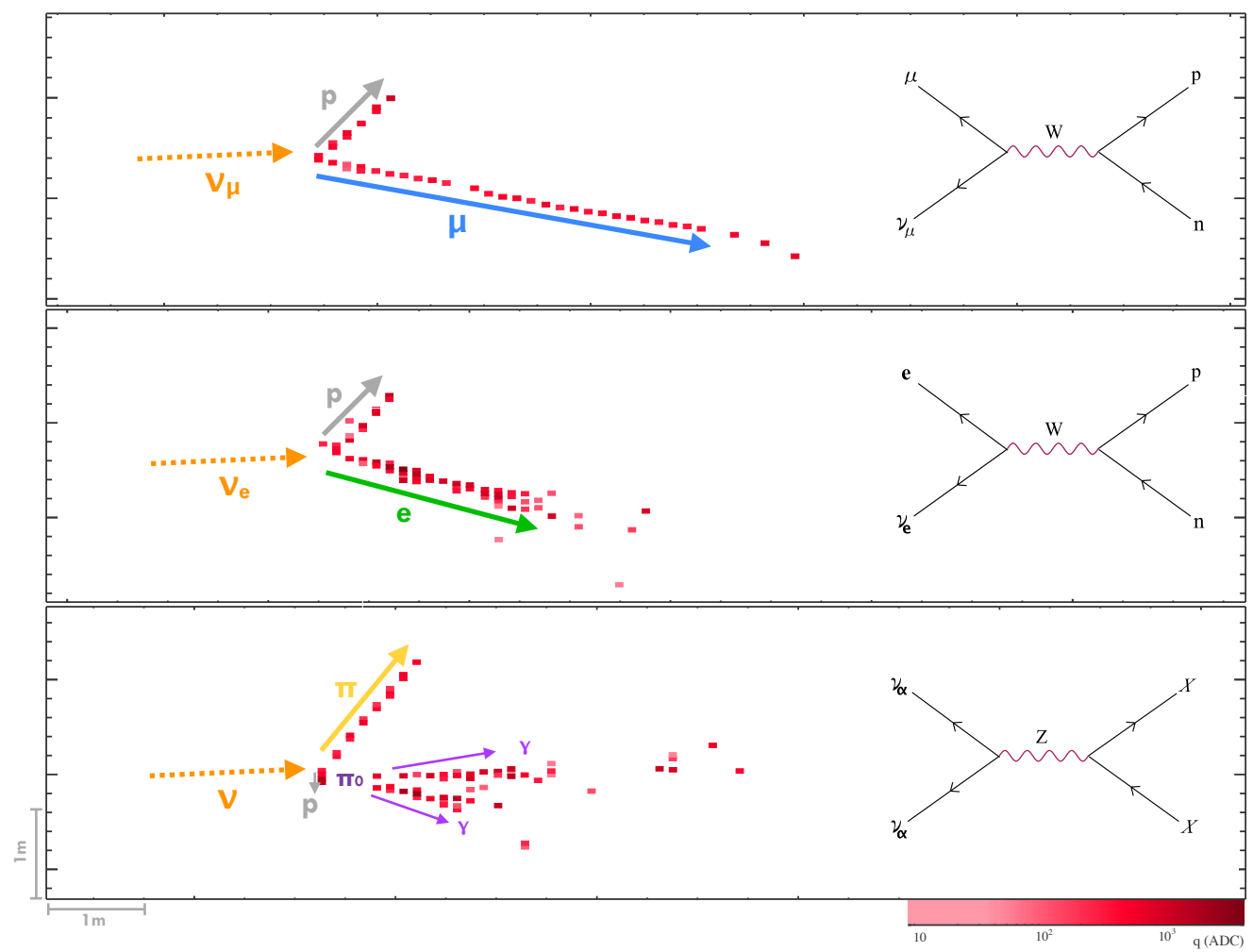

Figure 5.1: $\nu_{\mu}$ CC neutrino interaction with long muon (top). $\nu_{e}$ CC neutrino interaction with electron shower (middle), Neutral current (bottom). 
readout windows, whereas the ND is expected to separate $\sim 5$ neutrino interactions in each 10microsecond neutrino beam window. The beam spill (the length of time within which we expect neutrino interactions from the beam to occur) is only within the window of 10 microseconds and the time outside the beam spill allows us to do background determination. All those collected hits can be divided into two groups of hits: signal and noise. To separate accurately the signal hits from the noise hits and further separate the signal hits into clusters of hits that originate from different sources, we use an expanding, density-based clustering algorithm (DBSCAN) described in [79] which makes use of space and time information. In the DBSCAN algorithm, there are two types of points: core and border. The minimum number of neighbors (pairs whose distance is below a threshold) is defined as core points whereas border points have less than the minimum number of neighbors, but are allowed to be included in the group of hits (clusters) if and only if they are the neighbor of a core point. The algorithm makes clusters by expanding the cluster around the core points. Points that do not belong to these categories are treated as noise. This algorithm takes as input a pair of cell hits and calculates a distance metric in space and time defined as

$$
\epsilon=\left(\frac{|\Delta T|-|\Delta \vec{r}| / c}{T_{\mathrm{res}}}\right)^{2}+\left(\frac{\Delta Z}{D_{\mathrm{pen}}}\right)^{2}+\left(\frac{\Delta X \mathrm{or} Y}{D_{\mathrm{pen}}}\right)^{2}
$$

where $T_{\text {res }}$ is the timing resolution for the quadratic sum of two hits (time resolution for FD is $\sim$ $10 n s$ and for ND is $\sim 5 n s$ ), $D_{\text {pen }}$ is a distance penalty, $\Delta T$ is the time between two hits (order of nanoseconds), $\Delta Z$ and $\Delta X$ or $Y$ are the distance between hits in each view ( in $\mathrm{cm}$ ). The hits which occurred in the same view the distance can be written as: $|\Delta \vec{r}|=\sqrt{\Delta Z^{2}+\Delta X \text { orY } Y^{2}}$, however, for hits in opposite views $|\Delta \vec{r}|=\Delta Z$.

The slicing algorithm, called "Slicer", requires that each slice contain a single interaction. The performance of the slicer is determined from two metrics: efficiency and purity.

$$
\begin{gathered}
\text { Efficiency }=\frac{\text { Energy deposited in slice from interaction }}{\text { Total energy deposited in slice from interaction }} \\
\text { Purity }=\frac{\text { Deposited energy in slice from interaction }}{\text { Total energy in slice }}
\end{gathered}
$$

In FD, simulation of cosmic events, slicing was found to have an efficiency and purity of $99.3 \%$, whereas for the ND neutrino simulations slicing had an efficiency of $94.4 \%$ and a purity of $98.5 \%$ $[80]$.

\subsubsection{Identifying Lines with Multi-Hough Transform}

The next step after slicing is to identify lines in each slice using a modified Hough transform algorithm [81]. This algorithm takes as input pairs of points characterized by a straight line passing through them and parameterized in polar coordinate system, where $\rho$ gives the perpendicular distance from the line to the origin and $\theta$ gives the angle between $\rho$ and the $\mathrm{x}$-axis. The algorithm make lines and fits in each detector view separately. The line passing through each pair of hit points in the slice creates a Gaussian smear vote

$$
\text { vote }=e^{\frac{-\left(\rho-\rho_{0}\right)^{2}}{2 \sigma_{\rho}^{2}}} e^{\frac{-\left(\theta-\theta_{0}\right)^{2}}{2 \sigma_{\theta}^{2}}}
$$


where $\sigma_{\rho}=\frac{3}{\sqrt{12}}, \sigma_{\theta}=\frac{3}{d \sqrt{6}}$ and $\mathrm{d}$ is the distance between the two hits. A Hough map is created by filling the phase space with votes and the peak in the map is identified as the line of interest. To create new lines to the Hough map, we use an iterative method. First, we remove the last peak results of hough space and from the rest of the list, we look for a new hough map. This continues until we can not find any more peaks in a hough space above a certain, defined threshold. The metrics used to check the performance of this algorithm which delivers the dominant Hough lines that pass and form intersections close to the primary vertex of the slice.

The performance for the Far Detector is within a mean of $6.9 \mathrm{~cm}$ for neutral current, $4.1 \mathrm{~cm}$ for muon neutrino charge current, and $2.7 \mathrm{~cm}$ for electron neutrino charge current of the vertex whereas the mean distance for the secondary hough line is $9.9 \mathrm{~cm}$ for neutral current, $8.2 \mathrm{~cm}$ for muon neutrino charge current and $8.8 \mathrm{~cm}$ for electron neutrino charge current [80].

\subsubsection{Identification of Vertex using Elastic Arms}

After slicing and the Hough algorithm, the next reconstruction stage is to run an elastic arm algorithm on each slice to find the primary neutrino interaction point. The output of the algorithm is a global 3D vertex point by seeding the lines made by the Hough algorithm. The single point in a slice where the prong arms meet is the vertex. An "elastic arms" (also called as "deformable templates") is a straight line defined by polar angle $\theta_{a}$ and azimuthal angle $\phi_{a}$ such that the location of the arm originated at $\left(x_{0}, y_{0}, z_{0}\right)$ with a distance $\mathrm{s}$ in Cartesian coordinates is

$$
\begin{aligned}
& x(s)=x_{0}+s \sin \theta_{a} \cos \phi_{a}, \\
& y(s)=y_{0}+s \sin \theta_{a} \sin \phi_{a}, \\
& z(s)=z_{0}+s \cos \theta_{a} .
\end{aligned}
$$

To best describe the event topology, the Elastic Arm algorithm [82] finds the parameters $\left(x_{0}, y_{0}, z_{0}, \vec{\theta}, \vec{\phi}\right)$ by minimizing an energy function of the form

$$
\mathrm{E}=\sum_{i=1}^{N} \sum_{a=1}^{M} V_{i a} M_{i a}+\lambda \sum_{i=1}^{N}\left(\sum_{a=1}^{M} V_{i a}-1\right)^{2}+\frac{2}{\lambda_{\nu}} \sum_{a=1}^{M} D_{a}
$$

where $M$ and $N$ are the total number of arms and hits in slice, respectively. $M_{i a}$ gives distance that from cell hit $i$ to arm $a$. This is computed as the perpendicular distance from the hit $i$ to the projection of the arm in the detectors two $2 \mathrm{D}$ views, given by

$$
\mathrm{M}_{i a}=\left(\frac{d_{i a}^{\mathrm{perp}}}{\sigma_{i}}\right)^{2}
$$

$\sigma_{i}$ is a normalized factor (half of the cell depth $/ \sqrt{12}=0.9 \mathrm{~cm}$ ), $V_{i a}$ is the likelihood that hit $\mathrm{i}$ associated with arm a is assumed proportional to $e^{\left(-\beta M_{i a}\right)}$ and the noise is assumed to be a constant factor $e^{(-\beta \lambda)}$

$$
\mathrm{V}_{i a}=\frac{e^{\left(-\beta M_{i a}\right)}}{e^{(-\beta \lambda)}+\sum_{b=1}^{M} e^{\left(-\beta M_{i a}\right)}} .
$$

In the above equation, we have defined $D_{a}$ to be the distance from the vertex to first hit on the arm $a, \beta$ is the range of influence, and the penalty terms are controlled by $\lambda$ and $\lambda_{\nu}$. The first 
term in Eq. 5.8 evaluates the goodness of fit between the arms and the hits, which minimizes when arm passes through the hits. When any arm does not contain any hit that is treated as a penalty as mentioned in the second term. The third one, the penalty term for which the vertex position is far from the first hit. This term is very important while finding a vertex position for NC events where two photons are produced from $\pi^{0}$ after traveling some distance in the NOvA detector. The likelihood for a photon that propagate with a distance d before converting is proportional to $e^{\left(d \lambda_{\nu}\right)}$, where $\lambda_{\nu}=7 / 9 X_{0}(30 \mathrm{~cm})$, leads to a penalty term.

$$
\chi^{2}=2 \ln L=2 \frac{d}{\lambda_{\nu}}
$$

For all the vertices, arms are seeded and the directions are scanned and minimize the energy cost function mentioned in equation 6 . The minimization heavily depends on the performance of the multi-hough algorithm and uses ROOTs MINUIT class. The fit procedure is initialized with low values of $\beta$ to avoid local minima in the energy cost function, and $\beta$ is gradually tuned up to reach on the final vertex point in the slice.

The vertex resolutions of events tell us the performances of both Multi-Hough and Elastic Arms algorithms which is 11.6 (about 2 NOvA cells), 10.9 , and $28.8 \mathrm{~cm}$ for $\nu_{\mu} \mathrm{CC}, \nu_{e} \mathrm{CC}$, and NC events respectively.

\subsubsection{Prong Formation with Fuzzy k-Means}

The next step of the reconstruction chain is to consider the formation of prongs (clusters of hits with a start point and direction. We use a possibilistic fuzzy-k means algorithm [83, 84] for assigning a prong membership to each cell hit within the slice. The term "possibilistic" means the sum of each hits membership across all prongs is not required to be unity, which allows for outlier hits to be treated as noise. This algorithm works very well on separating the noise hits. The "fuzziness" allows a hit to belong to more than one prong. Fuzzy-k makes prongs separately in the XZ and YZ views using the cell hits in a slice, so it starts with a $2 \mathrm{D}$ view and furthermore it matches between the two views and produces 3D prong. The algorithm considers the vertex from Elastic Arms as the origin of the event in both views of the detector and the cell hits within the slice appear as peaks of deposited energy in a 1-D angular space around that vertex. Uncertainty is assigned as a function of distance from the vertex. The line connecting a cell hit to the vertex forms an angle with respect to z-direction of detector ranging from $-\pi$ to $\pi$. One can obtain the uncertainty associated with the angle, is modeled after the multiple scattering of $1-2 \mathrm{GeV}$ electrons and muons associated with each cell hit based on its distance from the vertex position. To find the prongs in angular space we seeded prong to find minima in dense cell hit, using the density matrix w:

$$
w_{k}=\sum_{i=1}^{n} e^{\left(-\frac{\theta_{k}-\theta_{i}}{\sigma_{i}}\right)^{2}}
$$

with

$$
\theta_{k}=\pi+\frac{k * \pi}{180}, 0 \leqslant k<360
$$

Associating each cell hit with a prong starts with assuming there is only one prong centered on the densest cell hit region in angular space and then prong centers are added and updated using the 
iterative method. The distance from each cell hit $j$ to the prong center $i$ is calculated as

$$
d_{i j}=\left(\frac{\theta_{i}-\theta_{j}}{\sigma_{i}}\right)^{2}
$$

and the prong membership is assigned with

$$
U_{i j}=e^{-\frac{m \sqrt{a} d_{i j}}{\beta}}
$$

where $a$ is the number of prong centers in the slice. $m$ is a measure of fuzziness of prongs and is set to 2 to allow membership to be shared between prongs. $\beta$ is a normalization factor which represents the expected spread of hits around the prong center. Furthermore, the prong centers are updated with:

$$
\theta_{i}^{\prime}=\theta_{i}+\frac{\sum_{i=1}^{n} \frac{U_{i j}^{m}}{\sigma_{j}}\left(\theta_{j}-\theta_{i}\right)}{\sum_{i=1}^{n} \frac{U_{i j}^{m}}{\sigma_{j}}}
$$

Prong angles are updated and additional prongs are added until all cell hits have at least a $1 \%$ membership in a prong at the maximum number of prong seeds has been reached. Prongs with significant membership overlaps are merged. Prongs with large spatial gaps that indicate two colinear particles are split.

At the end of the prong formation stage, there is a set of $2 \mathrm{D}$ prongs for each view of the NOvA detector. The next stage in the process is two match prongs between views in order to form 3D prongs. Matching involves comparing the energy profile of a prong in each view. A Kuiper metric $K=D^{+}+D^{-}$is used to find the best match for the prong, where $D^{+}=\max \left(E^{X Z}(s)-E^{Y Z}(s)\right)$ and $D^{-}=\max \left(E^{Y Z}(s)-E^{X Z}(s)\right)$ are the largest negative or positive distances between the profiles respectively (Fig. 5.2 and 5.3).

The performance of this algorithm is based on the completeness of hits produced by the primary lepton in charged current (CC) interactions. For $\nu_{e}$ CC events, the average completeness is $88 \%$ : $95 \%$ for quasi-elastic events and $86 \%$ for non-quasi-elastic events. For $\nu_{\mu} \mathrm{CC}$ events, these numbers are $93 \%, 98 \%$, and $92 \%$ respectively.

\subsubsection{Kalman Track}

Another useful algorithm which reconstructs tracks from individual slices and is widely used in the NOvA's muon neutrino disappearance analysis is the Kalman algorithm [86]. This algorithm takes

input as clusters of hits formed from the Slicer algorithm and forms tracks in the two detector views (XZ and YZ) separately. Each view produces $2 \mathrm{D}$ tracks which are later matched to produce a single $3 \mathrm{D}$ track. To create $2 \mathrm{D}$ tracks, seeding is done where the seed is a segment of the track and formed from a pair of hits that are separated by less than 4 cells. The seed is propagated using a Kalman filter to extend the track and add any additional hits from the next cell using the current value of the track position and slope that are consistent with the track. Once a hit is added to the track the position of the track, direction, slope, and intercept is updated for the new measurement and the process is continued until no more hits can be added to the track. The propagation process starts from the downstream end of the detector, toward the upstream direction, because in the downstream end the particles emerging from the interaction should be the most separated from each 


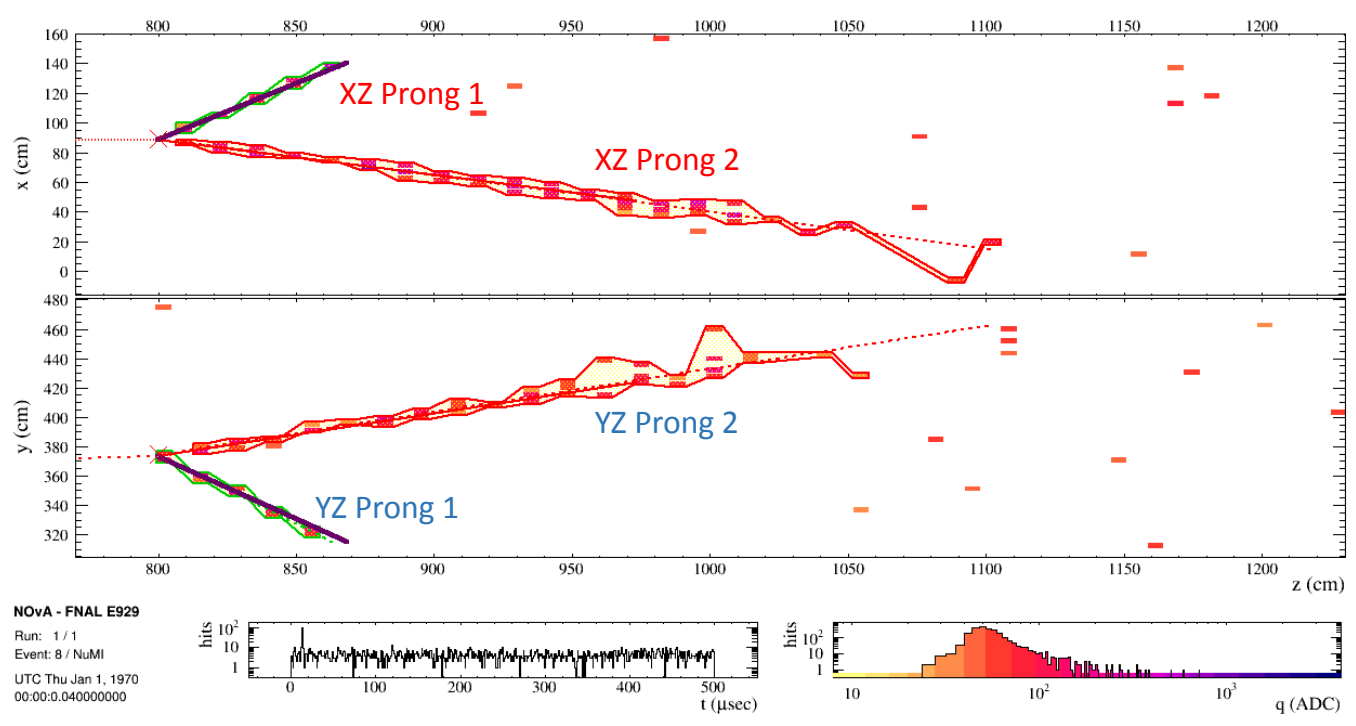

Figure 5.2: A completed 3D prong reconstruction using the fuzzy-k means algorithm for a simulated $\nu_{e} \mathrm{CC} \mathrm{QE}$ process in the FD. The red color shows the reconstructed prong as electron and green color shows as proton in $\mathrm{x}$ and $\mathrm{y}$ view. The corresponding cumulative energy profile histograms used to compute the suitable 3D prong matches is shown in Fig.5.3 [85].

other. Track propagation is continued as long as it did not get any consistent hit and the probability of a gap existing in a track from one hit to the next is less than 0.0001 . Once the track propagates to the upstream, propagation is reversed to go downstream to pick up any missing hits from the initial propagation. To find a good track, there is an optimization of track based on maximizing the efficiency of reconstructing long tracks (muons), with rejecting the poor reconstructed track.

Once all the 2D tracks have been made in each view independently, matching the two views of $2 \mathrm{D}$ track is based on the score metric which measures the overlap of $2 \mathrm{D}$ track in z-direction in both views. The score metric is defined as:

$$
\begin{aligned}
S & =\frac{\text { Start }_{\text {diff }}+\text { Stop }_{\text {diff }}}{\text { Length of Overlap in z-direction }}, \\
\text { Start }_{\text {diff }} & =\left|z_{\text {low of } x z \text { track }}-z_{\text {low of yz track }}\right| \\
\text { Stop diff }_{\text {d }} & =\left|z_{\text {high of xz track }}-z_{\text {high of yz track }}\right|
\end{aligned}
$$

Matching starts from the lowest value of $\mathrm{S}$ and progresses to higher values. The track merging process is performed iteratively until no more $2 \mathrm{D}$ tracks can be matched together to form a $3 \mathrm{D}$ track.

\subsection{Calibration}

The motivation of the calibration is to convert the registered signal into physically meaningful units of energy which ensures a uniform detector response within the entire detector. Cosmic ray muons are used to calibrate the NOvA detectors because they provide a source of consistent energy deposition through the entire detector. The first step in the calibration chain is to select the hits 

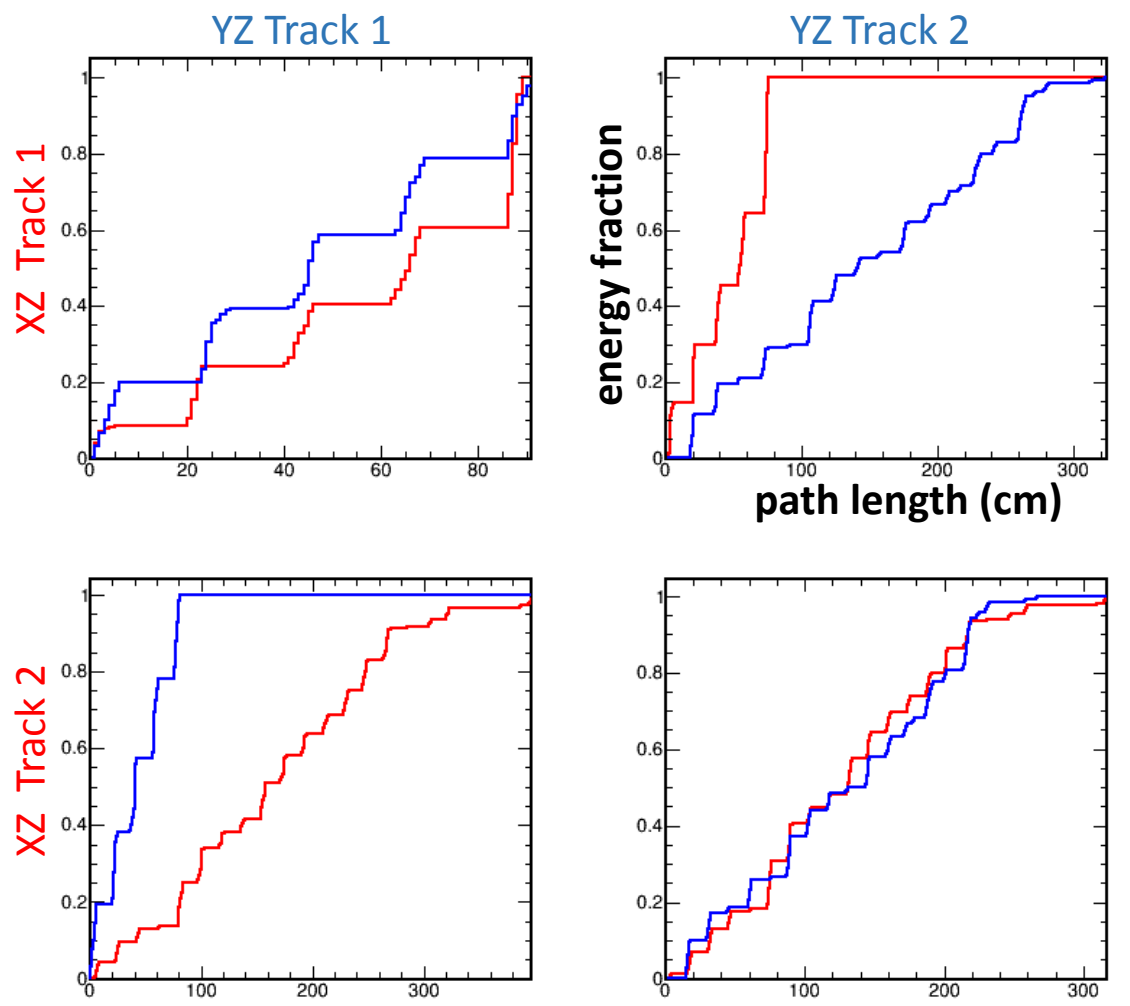

Figure 5.3: Cumulative energy profile as a function of path length along a prong for perspective 3D match candidates shown in Fig 5.2. The red and blue curves are the prongs are in the XZ view (vertical planes) and for the YZ view (horizontal planes) respectively. The upper-left and lower-right figure show the preferred matches with similar energy profiles that result in the green and red track respectively. The off-diagonal elements demonstrate the difference in energy profile shape for the wrong combinations [85]. 
associated with the reconstructed 3D muon cosmic muon rays using a CosmicTrack algorithm which straight line to all of the hits in the slice and rejects hits that are not consistent with that fit. A tri-cell criterion is also required to determine the path length of the track through the cell. Tri-cell is defined as a cell in which hits on a track also present in the adjacent upper and lower cells in the same plane as shown in Fig. 5.4. The calibration procedure is summarized from calibration technote [87].

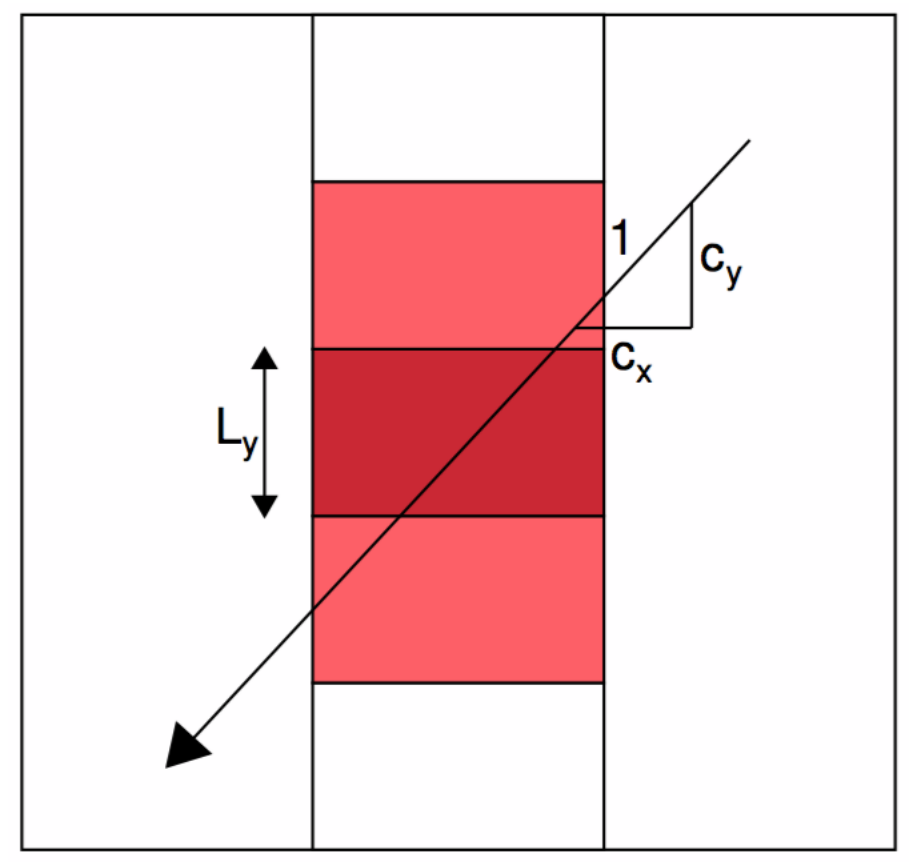

Figure 5.4: Selection of tri-cells associated with a cosmic ray muon. The dark red cell is a tri-cell because its neighbors are triggered by the same cosmic ray. The path-length in cell is given by $L_{y} / c_{y}$.

If there is not enough statistics to meet the tri-cell criterion (let's say having a bad channel next to a good channel), one can require that the hit in the adjacent cell was also hit in the planes. For corner cells, there is no requirement on the tri-cell criterion (no adjacent cell) but rather an average path length over all the directions that the track can have is used. A $2 \mathrm{D}$ plot of the mean response $(\mathrm{PE} / \mathrm{cm})$ from tri-cell vs $\mathrm{W}$ is drawn for each cell; where $\mathrm{W}$ is the distance from the readout, $\mathrm{W}=$ 0 is the center of the cell along the fiber, and a positive value of $\mathrm{W}$ is closer to the readout end. The profile of $2 \mathrm{D}$ plot is taken and a cell-by-cell attenuation is performed after applying a threshold and shielding corrections. The threshold corrections refer to a minimum number of hit requirement for reaching APD as it slightly upwards fluctuation in the number of photons produced by the energy deposition. Shielding is defined as the average visible energy depositions from minimum ionizing particles (MIPs) are not truly spatially uniform in the detector due to its own mass of the detector. To account for these two effects a correction factor is applied in each cell defined as :

$$
\mathrm{T}=\frac{\mathrm{PE}}{\lambda} \cdot \frac{\mathrm{E}_{\text {True }}}{\mathrm{E}_{M I P}}
$$

where $\mathrm{T}$ is the combined threshold and shielding correction factor, $\mathrm{PE}$ is the number of simulated 
photo-electrons registered by electronic readout, $\lambda$ is the number of simulated photons seen at the readout without fluctuations, $\mathrm{E}_{\text {True }}$ is the true energy deposited in the cell and $\mathrm{E}_{M I P}$ is the energy expect to be deposited and it depends on the path length through the cell.

\subsubsection{Attenuation Correction}

Light in the detectors is attenuated as it travels through the wavelength shifting (WLS) fiber which is used to transport the light produced by a charged particle traversing the scintillator to the APD. Attenuation calibration corrects for the amount of energy deposited in the detector and registered by an APD can be expressed as PECorr which is the corrected number of photo-electrons irrespective of the position of energy deposition. The $\mathrm{ADC} / \mathrm{cm}$ is recorded for a given cell as a function of $\mathrm{W}$ in a 2D histogram after applying a correction factor from threshold and shielding effect. The profile of the $2 \mathrm{D}$ histogram is constructed by taking the median value in each $\mathrm{W}$ bin and is fit to a double exponential function considering both short and long path light traveling as of the form:

$$
\mathrm{y}=C+A\left(e^{\frac{\mathrm{W}}{\mathrm{X}}}+e^{-\frac{\mathrm{L}+\mathrm{W}}{\mathrm{X}}}\right)
$$

Where $\mathrm{L}$ is the total length of the cell, $\mathrm{C}, \mathrm{A}$, and $\mathrm{X}$ are free parameters. $\mathrm{X}$ gives the cell attenuation length as well. The fit includes central part of the cell which includes the range from $[-150,150] \mathrm{cm}$ for Y view ND Muon Catcher cells and active cells, $[-150,50] \mathrm{cm}$ for X view ND Muon Catcher cells. The hits close to the beginning and the end of the cell show different feature than from the lion's share of the cell. This is as a consequence of the light that hits the white PVC cell walls is reflected back into the scintillator. The top of the cell is composed of a black plastic which is not nearly as reflective and results in a greater light loss. To correct for this effect, a polynomial function is added to the above exponential form at the two ends of the cell. The roll-off is empirically defined as:

$$
\mathrm{y}= \begin{cases}1-\alpha_{R}\left(W-W_{R}\right)^{4}, & \text { if } W>+W_{R} \\ 1, & \text { otherwise } \\ 1-\alpha_{L}\left(W-W_{L}\right)^{4}, & \text { if } W>-W_{L}\end{cases}
$$

This is another case where large residuals are observed in some cells. This is because of fiber position variation within the cell. Normally the fiber lies in the corners of the cell, but if it somehow rises into the central part of cell, then it collect more light than expected resulting in these residuals. In these cases, the exponential function does not correctly describe the response. A LOWESS (Locally Weighted Scatter plot Smoothing) fit is done using a tricube weight,

$$
\mathrm{w}_{i}= \begin{cases}\left(1-\left|\frac{W-W_{i}}{\sigma}\right|^{3}\right)^{3}, & \text { if }\left|W-W_{i}\right|<\sigma \\ 0 & \text { if }\left|W-W_{i}\right| \geq \sigma\end{cases}
$$

where $W_{i}$ is the $i^{t h}$ neighbor point around local point. $w_{i}$ is the weight on $W_{i}$. W is the local point on the curve. $\sigma$ is $30 \mathrm{~cm}$ is the range of measurements that influence the value of $\mathrm{W}$. An example of full attenuation fits for the NOvA ND is shown in Fig.5.5. Fig. 5.6 shows examples of before and after calibration for an horizontal and vertical cell view for ND. The attenuation calibration takes all the above conditions to smooth the cell response. 
ND cosmic data - plane 90 (horizontal), cell 79

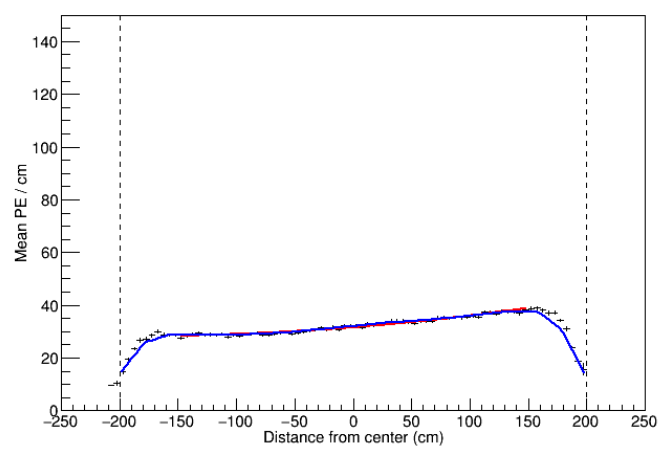

ND cosmic data - plane 89 (vertical), cell 78

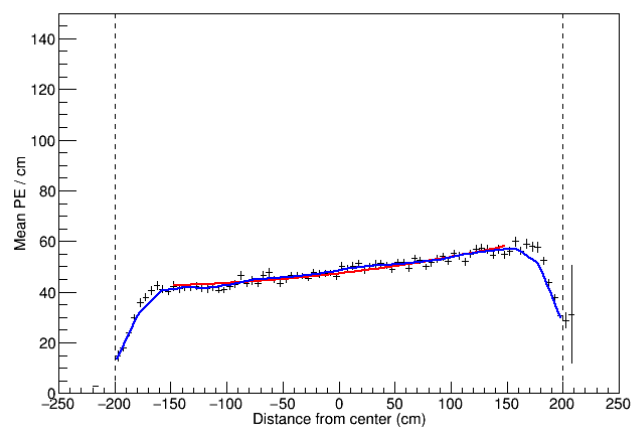

Figure 5.5: An examples of good attenuation fits with LOWESS corrections for ND. The blue curve is the full attenuation fit including the LOWESS fit and the red curve is the double exponential fit. The left and right plot is for horizontal and vertical view cells respectively.
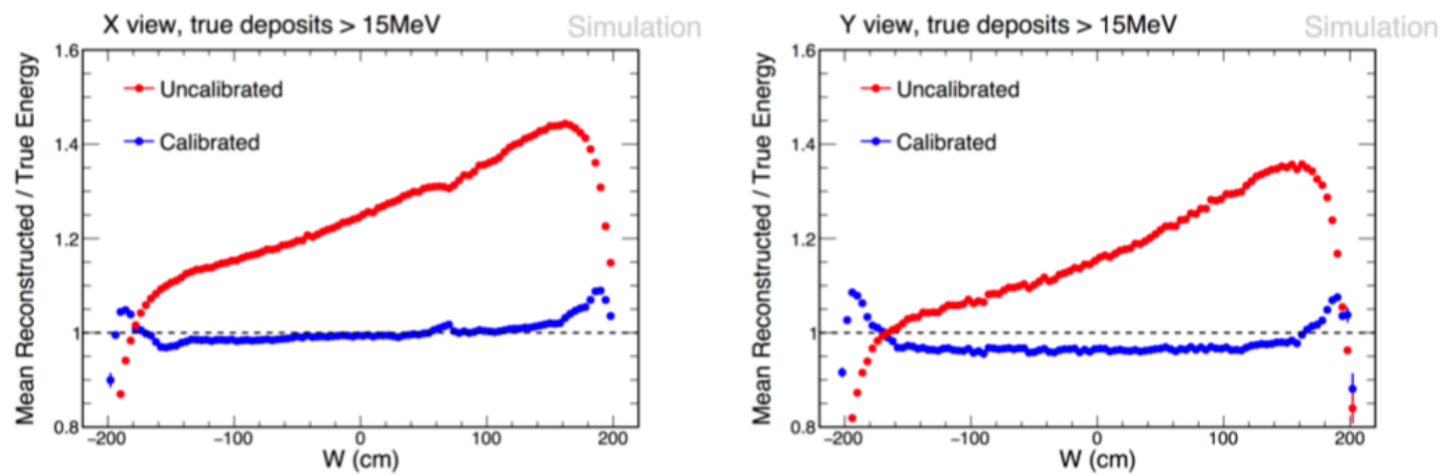

Figure 5.6: An examples of ratio between mean reconstructed to true energy vs W. The red and blue dots are before and after calibration applied respectively. The left and right plot is for horizontal and vertical view cells respectively. 


\subsubsection{Absolute Energy Scale}

The main goal of the absolute energy calibration is that it takes the input as relative corrected scale, measured in arbitrary units called PEcorr/cm, to a physical energy scale measuring unit in $\mathrm{GeV} / \mathrm{cm}$. The absolute calibration also uses stopping cosmic ray muons instead of passing through the entire detector as well as the tri-cell criterion. Stopping muons are identified by the presence of Michel electron at the end of the muon track due to its decay $\left(\mu \rightarrow e^{-}+\bar{\nu}_{e}+\nu_{\mu}\right)$ and it is well understood from the Bethe-Bloch formula. The Bethe-Bloch equation is applied in order to estimate how much energy is lost via ionization inside the scintillator and it ignores the energy deposition through PVC material. In the NOvA liquid scintillator (chains of polyethylene), expected energy loss per unit length due to a minimum ionizing particle is $1.79 \mathrm{MeV} / \mathrm{cm}$. The energy depositions occurring between 100 and $200 \mathrm{~cm}$ from the end of a track are considered to be within the MIP region in the detector which is used for determining the absolute energy calibration. The determination of scale factor for absolute calibration is the ratio between the mean of the muon energy unit $\mathrm{MEU}_{\text {truth }}$ to mean of the $\mathrm{MEU}_{\text {reco. }}$ MEU is the mean of the detector response in units of PECorr (or simulated energy deposition in $\mathrm{MeV}$ ) to a stopping muon tri-cell hit within the track window $(100-200 \mathrm{~cm})$ divided by the path length. The corrected photo-electrons per length (PECorr $/ \mathrm{cm}$ ) distribution between the data and simulation is shown in Fig. 5.7. The scale factor is used to convert the PECorr $/ \mathrm{cm}$ in data to $\mathrm{MeV} / \mathrm{cm}$. In this way, we calibrate NOvA detectors independently and the constant is stored in the database, also they stored for different time periods so that a PE signal in raw digit object is converted to energy unit.
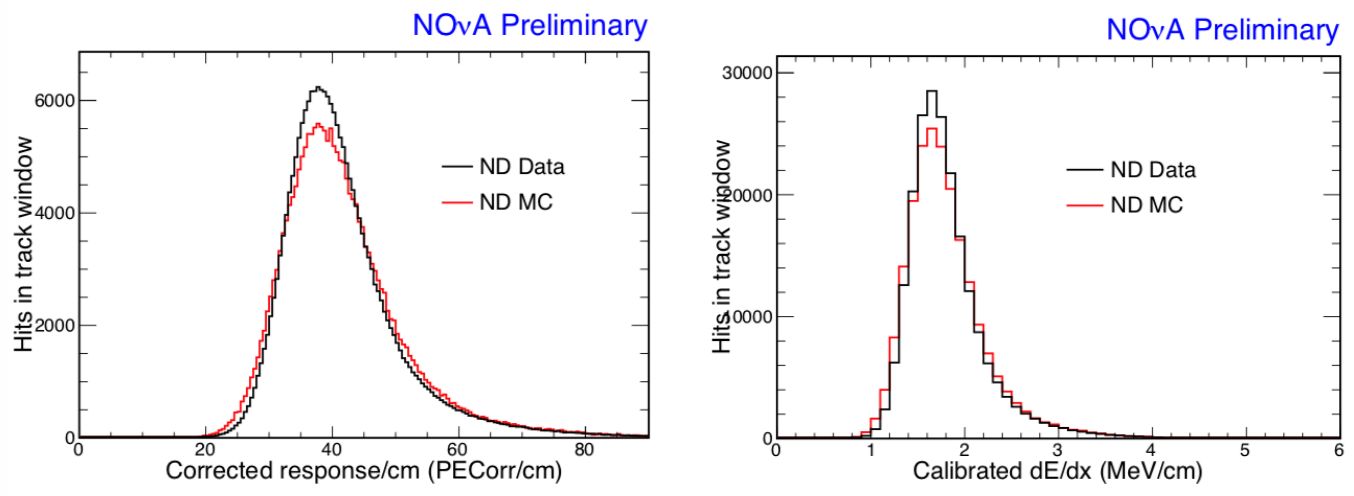

Figure 5.7: Corrected detector response as a function of distance from the track end in ND data and MC (left). Calibrated energy distribution per unit path length as a function of distance from the track end in ND data and MC (right). 


\section{Chapter 6}

\section{Measurement of Inclusive $\nu_{\mu} \mathbf{C C}$ Cross-section}

In current and future long-baseline neutrino oscillation experiments, beams of (anti)muon-neutrinos are used to precisely measure the rate of (anti)muon-neutrino disappearance and (anti)electronneutrino appearance. The accuracy of these measurements depends explicitly on how well we are able to measure the energy of the incoming neutrino, a quantity that is estimated from the lepton and hadrons visible in the final state after the neutrino has interacted. To relate these final state observables to the energy of the neutrino, accurate knowledge of neutrino-nucleon interaction crosssections and the dynamics of the propagation of particles through the nuclear matter are necessary. Many of the uncertainties on oscillation parameters that arise from our limited understanding of neutrino cross-section are reduced by using a Near Detector that is functionally identical to the Far Detector. However, the near and far detectors are substantially different in size and have different acceptance of the neutrino beam. Correctly accounting for these acceptance effects also requires knowledge of the neutrino cross-section.

Therefore, understanding and modeling of (anti)neutrino-nucleus scattering are critical for these precise oscillation measurements. Charged-current (CC) interactions, where a charged-lepton is in the final state, are used to measure 3-flavor oscillations probabilities since the final state lepton identifies the neutrino flavor and the energy of the neutrino can be reconstructed. Therefore, predicting the kinematic distributions of the final state lepton is critical. Such predictions rely on a-priori knowledge of neutrino-nucleus cross-sections. As shown in Fig. 6.1, neutrino-nucleus charged-current inclusive cross-section in the energies relevant for NOvA has 10-20\% uncertainties.

\subsection{Analysis Overview}

The flux-integrated double-differential cross-section with respect to the final-state muon's true kinetic energy and true angle is:

$$
\left(\frac{d^{2} \sigma}{d \cos \theta_{\mu} d T_{\mu}}\right)_{i}=\frac{\sum_{j} U_{i j}\left(N^{\mathrm{sel}}\left(\cos \theta_{\mu}, T_{\mu}\right)_{j}-N^{\mathrm{bkg}}\left(\cos \theta_{\mu}, T_{\mu}\right)_{j}\right)}{\epsilon\left(\cos \theta_{\mu}, T_{\mu}\right)_{i}\left(\Delta \cos \theta_{\mu}\right)_{i}\left(\Delta T_{\mu}\right)_{i} N_{\text {target }} \phi}
$$




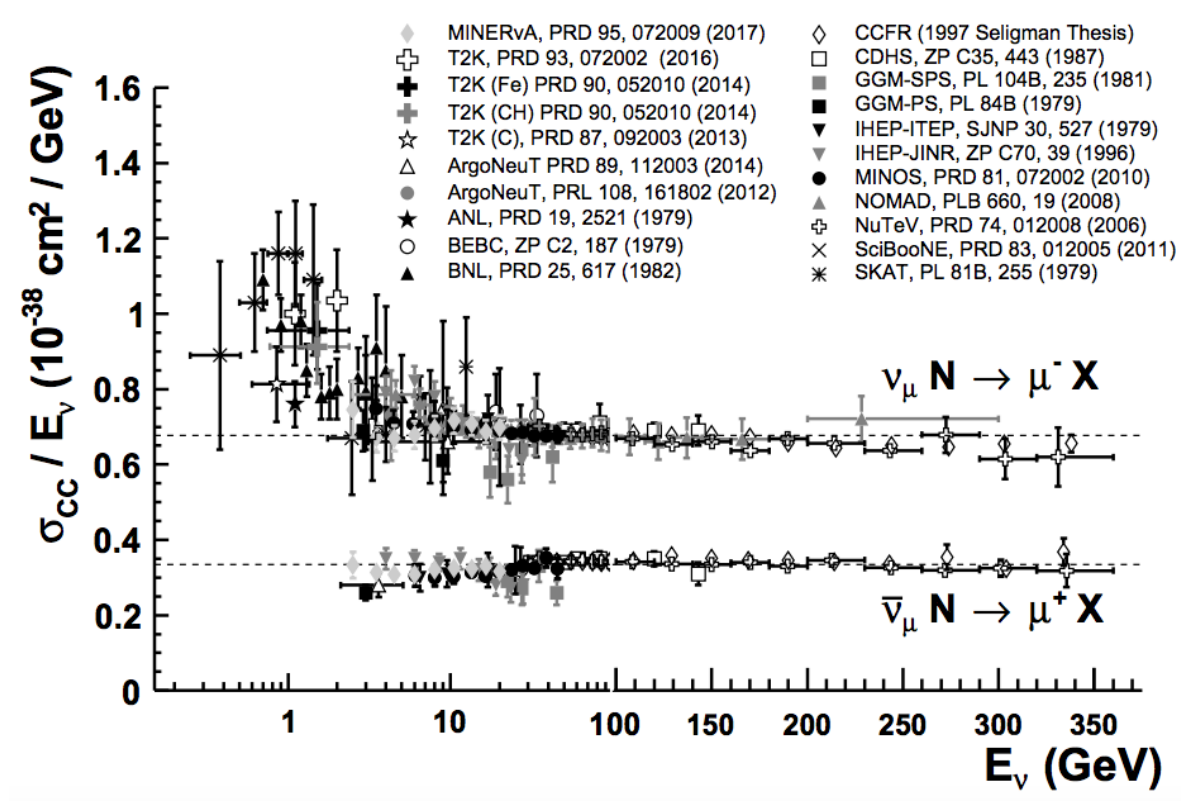

Figure 6.1: Neutrino charged-current inclusive cross-section measurements as a function of neutrino energy $[88]$

Here, $N^{\text {sel }}$ is the number of selected events, $N^{\text {bkg }}$ is the estimated number of background events, and $U$ is the unfolding matrix that corrects the reconstructed quantities for detector resolution/smearing and maps the reconstructed quantities to true quantities. $\phi$ is the neutrino flux, $\epsilon$ is signal selection efficiency and $N_{\text {target }}$ is the number of nucleon targets in the fiducial volume.

The measurement of the inclusive cross-section in bins of true neutrino energy is made over the range of muon kinematics used in the double-differential measurement in Eq. 6.1:

$$
\sigma\left(E_{i}\right)=\frac{\sum_{j} U_{i j}\left(N^{\mathrm{sel}}\left(E_{j}\right)-N^{\mathrm{bkg}}\left(E_{j}\right)\right)}{\epsilon\left(E_{i}\right) N_{\mathrm{target}} \phi\left(E_{i}\right)}
$$

The signal in these measurements is defined as all $\nu_{\mu} \mathrm{CC}$ interactions where the true interaction vertex is in the fiducial volume. All interactions other than $\nu_{\mu} \mathrm{CC}$ or those $\nu_{\mu} \mathrm{CC}$ interactions that occur outside of the fiducial volume are regarded as background. In this systematics-limited measurement, we use the minimization of the systematic uncertainty as the figure of merit (FoM) to determine selection criteria. We therefore describe the treatment of the dominant systematic uncertainties in this analysis in Section 6.2, followed by a description of the signal selection criteria in Section 6.3. Section 6.4.4 describes an updated muon and neutrino energy estimator and resolution studies used to determine the binning in this analysis. The measurement will be expressed as a function of true quantities, and unfolding techniques will be used to map reconstructed quantities to true space. The unfolding studies done for this analysis are discussed in Section 6.6. Acceptance efficiencies, flux and target count calculations are detailed in Sections 6.5, 6.7 and 6.8 respectively. Section 6.9 discusses some additional systematics that are not considered in Section 6.2, and summarizes the final systematic uncertainties on the measurement. Further technical details can be found in [89]. 


\subsubsection{Simulation, Reconstruction and Data set details}

The simulation and reconstruction used in this analysis are identical to that used for the 2018 oscillation results. The relevant reconstruction chain includes slicing events [90] and the reconstruction of particle trajectories with the KalmanTracker algorithm[91] and showers with the FuzzyK and LID reclustering algorithms[92].

The data being analyzed for this measurement were taken from August 16, 2014 to February 20, 2017 in the Near Detector and are equivalent to $8.09 \times 10^{20}$ POT exposure. During this period, the beam power increased from $250 \mathrm{~kW}$ to $560 \mathrm{~kW}$ to $700 \mathrm{~kW}$. The simulation used is run and intensity matched, real conditions Monte-Carlo, where real data taking conditions are simulated.

This analysis has been performed using the Common Analysis Frame (CAF's) and the CAFAna framework [93]. This required development of some aspects of the framework necessary for a crosssection analysis.

\subsection{Systematic Uncertainties}

Systematic uncertainties are assessed using systematically shifted simulation samples, where available and re-weighting events in other cases. The cross-section is completely recomputed, including background estimation, unfolding, efficiency correction, and flux normalization, for each systematic effect. The sources of systematic uncertainties considered in this analysis are discussed below.

\subsubsection{Flux}

The flux uncertainty due to the hadron production mis-modeling in the beamline is assessed using the PPFX multi-universe approach [94]. Each data point, such as the total cross-section for an interaction or a differential production of a particle, is a parameter. In each universe, each parameter has a value obtained by randomly sampling in a multi-dimensional Gaussian distribution centered on the default parameter values with covariances to account for the uncertainties and correlations coming from the data. PPFX calculates the correction of the default MC model per universe and provides these values (weights) per neutrino. Then, they are stored and propagated associated to corresponding neutrino interaction. The resulting weights are used to compute the variance for any kinematic variable correcting the expected neutrino yield.

Figure 6.2 shows the fractional uncertainty respect to the neutrino energy, regardless of any bin to bin correlations, split into the PPFX categories. The black line represents the total, calculated using the CAFAna tools (described in 6.7). The contributions from different categories are also placed there but calculated using PPFX directly on the G4NuMI ntuples (we do not propagate them through CAFAna). The total uncertainty around the focusing peak is approximately $8 \%$ mostly coming from pions created when the primary proton beam interacts with the target, nucleon interactions outside the target and interactions with incident mesons. A detailed description of each category is provided in [94].

The uncertainty in the flux due to the beamline geometry mis-modeling is taken from the work described in [95]. This uncertainty is accounted for by the oscillation analyses as a function of neutrino energy. However, for this analysis, this is computed as a function of the neutrino 3-momentum to retain the resulting uncertainty on the beam's angular distribution. This is especially important 


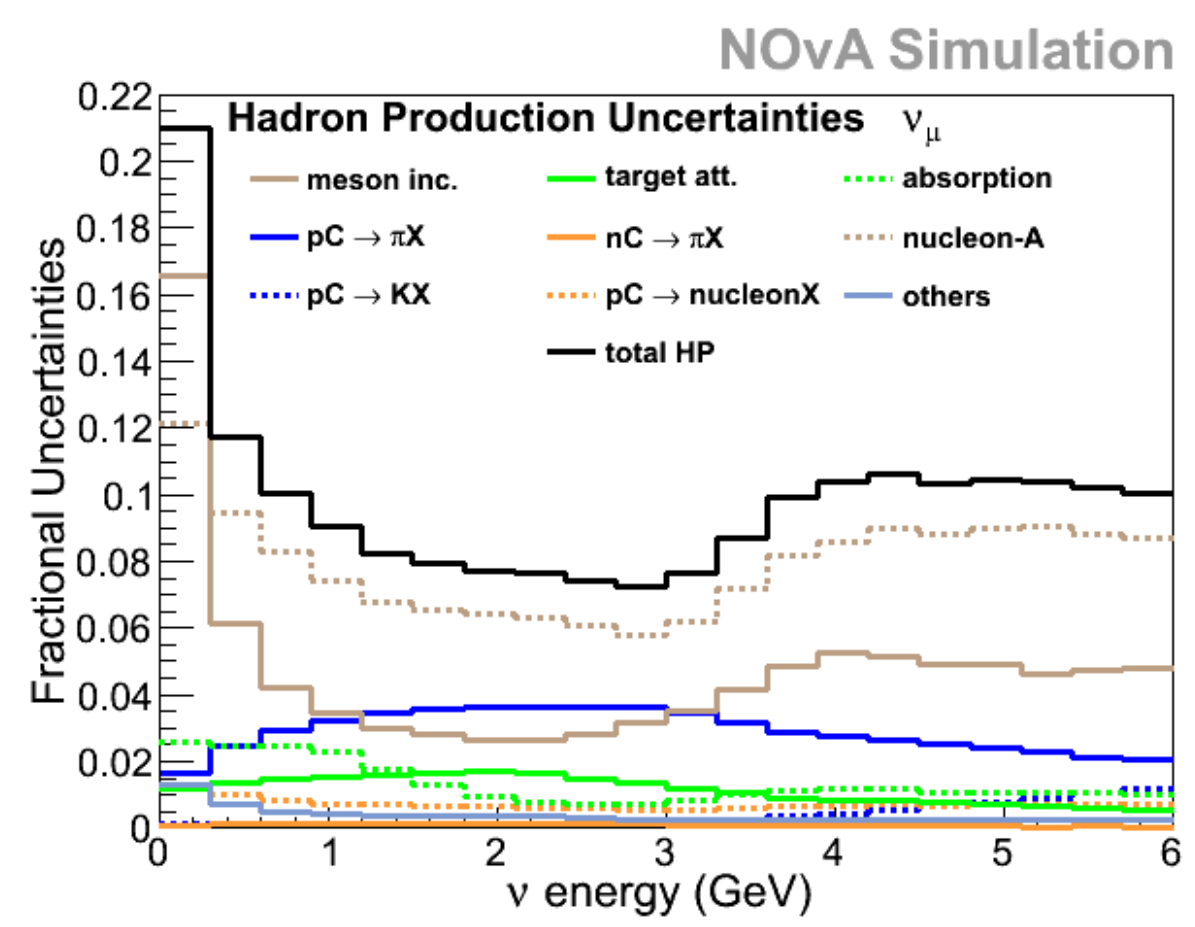

Figure 6.2: Total hadron production systematics (in black) split into categories handled by PPFX.

in the double-differential cross-section measurement where a significant discrepancy is observed between data and $\mathrm{MC}$ in the reconstructed angle of the muon with respect to the beam direction. This uncertainty is approximately $4 \%$ around the peak and it is treated as totally uncorrelated to the hadron production uncertainty.

\subsubsection{Neutrino-nucleus interaction modeling}

Neutrino-nucleus interaction modeling uncertainties impact the shape and normalization of both the signal and background distributions in every bin. To assess the impact these uncertainties have on the cross-section measurement, we make use of the GENIE weights [96]. In particular, we use a "multi-universe" approach where, for each 'universe', each GENIE reweightable parameter is shifted by a random fraction of sigma (generated from a normal distribution centered at zero) and the event is reweighted according to these shifts. A cross-section is determined for each universe using the analysis procedure described above. The width of the distribution of differences between each universe and the nominal cross-section result is used to determine the systematic uncertainty associated with the neutrino-nucleus interaction modeling. The upper and lower boundaries of the systematic error band are determined such that $34 \%$ of the universes are enclosed in the nominal value and the upper and lower boundaries. Details can be found in [97].

In addition to GENIE systematic, the near detector data are used to improve the simulation of the various kinematic distributions. The tuning is primarily focused on MEC events which lie somewhere between $\mathrm{QE}$ and RES region in $Q^{2}$. The details about the cross-section weights are discussed in the [98].

In GENIE, the quasi-elastic cross-section is calculated using the single nucleon dipole form factor 
that is constrained by deuterium data. This single nucleon cross-section is then corrected for the effects of Pauli blocking and the random phase approximation (RPA). The axial mass $M_{A}$ for the charged-current quasi-elastic cross-section to $1.04 \mathrm{GeV} / \mathrm{c}^{2}$ based on error-weighted mean from different bubble chamber experiments such as ANL, BNL, and FNAL experiment (previously it was set $0.99 \mathrm{GeV} / \mathrm{c}^{2}$ ). The effect of this change is to increase the normalization of the quasi-elastic events by $5 \%$ with respect to the previous analyses.

We also apply the RPA suppression as a function of $Q^{2}$ given to us by MINERvA experiment, both for central value and systematics for QE events. However, RPA influences both QE and RES events and mostly suppress at low $Q^{2}$ region. There is an absence of the theoretical model for RES RPA and work is currently under progress to improve calculations of this effect. At NOvA energies the pion production is through resonances, particularly at the lowest-lying one, such as $\Delta$ resonance region. The MiniBooNE and MINOS experiment observed the suppression of resonant pion production at low $Q^{2}$, provide circumstantial evidence for such a hypothesis. We have applied RPA suppression as a function low momentum transfer according to the calculation. We also observed there is significantly improved on the agreement between data and simulation of low momentum for forward going events. Fig. 6.3 shows the selected events in the near detector simulation with and without an RES RPA correction applied compared to the near detector data. The error band is drawn on the RPA model by taking the difference between our simulation with and without RPA model correction applied.
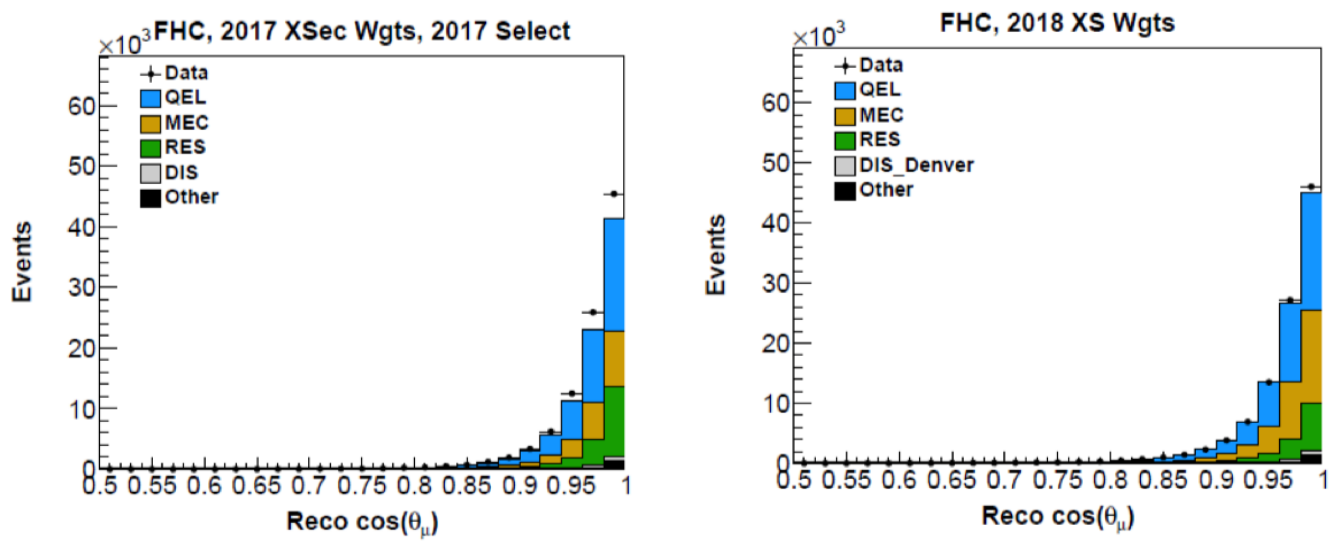

Figure 6.3: The left figure shows reconstructed cosine angle of muon without RES RPA correction applied, however right figure is with RES RPA correction applied (In 2017 cross-section weight does not have RES RPA and it is implemented in 2018 cross-section weight). In addition, MEC tune applied in both figures. The black bullets show the near detector data for $\nu_{\mu} \mathrm{CC}$ events. The disagreement between data and simulation in the forward going muons without RPA correction hints us that our data prefers for low- $Q^{2}$ suppression.

In NOvA, almost all DIS events occur in the 'transition DIS' region (at $\mathrm{W}=1.7 \mathrm{GeV}$ ), which is imperfectly modeled and understood. In this region, GENIE uses the DIS model for predicting DIS events. Above transition region DIS model is least reliable, however, below 1.7 GeV GENIE further modifies the Bodek-Yang model with a fit to additional data, and thus this region is more reliable. There is a tweak by $10 \%$ the central value of DIS events with $\mathrm{W}>1.7 \mathrm{GeV}$, with systematics there staying the same. The systematics are $50 \%$ for all DIS events for $\mathrm{W}<3 \mathrm{GeV}$, and for $\mathrm{W}>3 \mathrm{GeV}$ are $10 \%$.

For the MEC events, we have developed a $2 \mathrm{D}$ fit in true $q_{0}$, the visible hadronic energy in the 
event, and true $|\vec{q}|$ space, the three vector component of the four-momentum transfer to the hadronic shower. The optimization of 2D fit is done by comparing the reconstructed $q_{0}$ and $|\vec{q}|$ space, and the data-to-MC ratio in the reconstructed space is used as a weight to MEC events. The phase-space of true $q_{0}$ and $|\vec{q}|$ before and after applying the tuned weight is shown in Fig. 6.4.
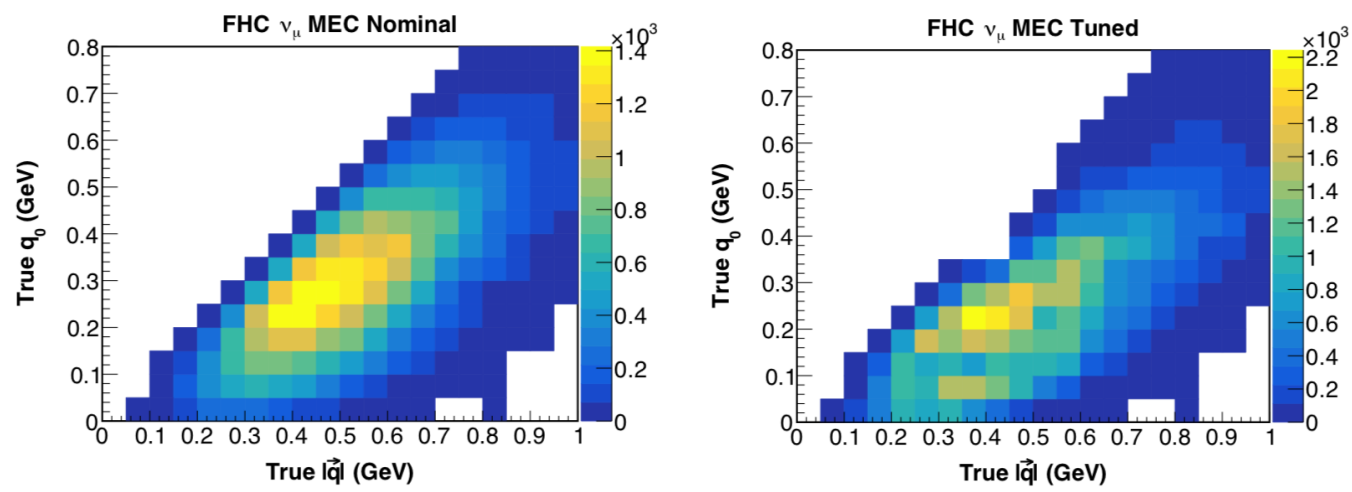

Figure 6.4: The true $q_{0}$ and $|\vec{q}|$ distribution in the nominal MEC simulation is shown on the left for forward horn current (neutrino mode) and the true $q_{0}$ and $|\vec{q}|$ distribution after it has been fitted in reconstructed space is shown in right figure.

The systematic error associated with the MEC events also very important, along with a central value tune. As we have discussed earlier that MEC events lie between QE and RES region in $Q^{2}$ and the error band is drawn on MEC events by systematically shifted the non-MEC events of our simulation and re-evaluating the fit between $q_{0}$ and $|\vec{q}|$ phase space. Altering eight knobs in MC for QE-like(more events at low energy, fewer at high) and RES-like (more events at higher hadronic energy, fewer at low) events, we re-fit the MEC events and these new fits are used as the \pm 1 $\sigma$ uncertainties for the MEC shape. The systematics that are altered to make the more QE-like scenario are:

- $\mathrm{QE} M_{A}:+1 \sigma$

- QE RPA Suppression : $+1 \sigma$

- QE RPA Enhancement : $+1 \sigma$

- QE Pauli Suppression : $-1 \sigma$

- $\operatorname{RES} M_{A}:-1 \sigma$

- RES $M_{V}:-1 \sigma$

- RES RPA suppression : $-1 \sigma$

- Calibration scale shifted : $-1 \sigma$

These knobs are used to alter to make the more QE-like scenario to achieve the systematics, however applying the knobs in opposite way to make the MEC fit more RES-like scenario. The visible hadronic energy distribution after applying MEC fit is shown in Fig. 6.5. 


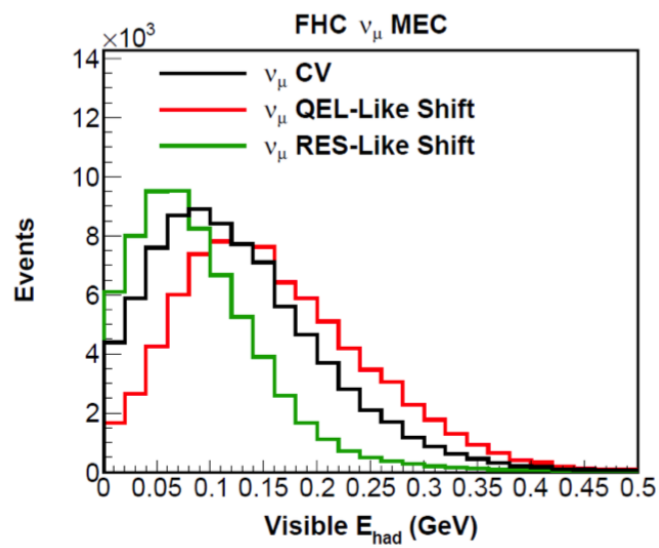

Figure 6.5: The visible hadronic energy distribution for fitted MEC events in the nominal procedure is in black line, the QE-like shift is in red, and more res-like shift is shown in green line

\subsubsection{Energy scale}

The uncertainty on the energy scale due to calibration is done using the same systematically shifted data sets that were used in the 2018 oscillation analyses. The energy scale uncertainty is assessed using systematically shifted simulation samples, where the absolute energy scale is shifted up and down by $5 \%$. It is also found that the calibrated energy response is not flat as a function of distance from the readout. This discrepancy is included as a calibration shape uncertainty, using a systematically shifted sample. The systematically shifted MC samples are:

1. prod_caf_R17-11-14-prod4reco.remid-

hotfix.b_nd_genie_nonswap_fhc_nova_v08_full_calib-shift-nd-xyview-neg-offset_v1

2. prod_caf_R17-11-14-prod4reco.remid-

hotfix.b_nd_genie_nonswap_fhc_nova_v08_full_calib-shift-nd-xyview-pos-offset_v1

3. prod_caf_R17-11-14-prod4reco.remid-

hotfix.b_nd_genie_nonswap_fhc_nova_v08_full_calib-shift-nd-func_v1

\subsubsection{Light Model}

The scintillator response model in the 2017 simulation includes Cherenkov light production. The uncertainty on the light model arises from the uncertainty on overall light yield of the scintillator and the efficiency with which Cherenkov photons are absorbed by the scintillator and re-emitted at wavelengths that can be detected. These uncertainties are assessed using systematically shifted MC samples to account for these effects:

1. prod_caf_R17-11-14-prod4reco.remidhotfix.b_nd_genie_nonswap_fhc_nova_v08_full_lightmodel-lightdown-calibup_v1

2. prod_caf_R17-11-14-prod4reco.remidhotfix.b_nd_genie_nonswap_fhc_nova_v08_full_lightmodel-lightup-calibdown_v1

3. prod_caf_R17-11-14-prod4reco.remidhotfix.b_nd_genie_nonswap_fhc_nova_v08_full_ckov-proton-shift-down_v1 


\subsection{Event selection}

In the event selection for this analysis, we begin with some basic event quality criteria to reject obvious backgrounds and ensure that there is a reconstructed Kalman track in the event. We next apply some loose fiducial and containment criteria based on reconstructed showers. With these criteria in place, we implement a new muon identification algorithm based on energy loss and scattering of reconstructed Kalman tracks and optimize a cut on a score provided by that algorithm. Finally, we optimize the fiducial and containment selection criteria with the muon selection applied.

A general strategy in this analysis is to determine selection criteria that minimize the systematic uncertainty of the total cross-section:

$$
\sigma=\frac{N_{\text {sel }}-N_{\text {bkg }}}{N_{\text {target }} \Phi \epsilon}
$$

where $N_{\text {sel }}$ is the number of selected events, $N_{\mathrm{bkg}}$ is the estimated number of selected background events, $\Phi$ is the total flux, $N_{\text {target }}$ is the number of targets and $\epsilon$ is the efficiency of the selection. Furthermore,

$$
\epsilon=\frac{N_{\text {sel,signal }}}{N_{\text {signal }}}
$$

Ignoring uncertainties on the flux and the number of targets (which depend very weakly on the selection criteria), and correlations between background estimates and efficiency, the fractional uncertainty on the cross-section is:

$$
\frac{\delta \sigma}{\sigma}=\sqrt{\frac{\left(\delta N_{\mathrm{sel}}^{\mathrm{stat}}\right)^{2}+\left(\delta N_{\mathrm{bkg}}^{\mathrm{stat}}\right)^{2}+\left(\delta N_{\mathrm{bkg}}^{\mathrm{syst}}\right)^{2}}{\left(N_{\mathrm{sel}}-N_{\mathrm{bkg}}\right)^{2}}+\left(\frac{\delta \epsilon}{\epsilon}\right)^{2}}
$$

In the studies that follow, the statistical uncertainty on the number of selected events is negligible and therefore ignored, and the fractional uncertainty on the total cross-section is plotted as either a function of a variable (eg, vertex position) or as a function of a cut value.

\subsubsection{Event Quality and Spill Cuts}

Spill cuts determine which NuMI spills are acceptable for analysis, which includes beam quality, electronics of the detector were performing properly. The spill cuts are:

1. Spill POT $>2 \times 10^{12}$

2. $-202<$ Horn current $<-198$

3. $-2.0<$ Horn $\mathrm{x}$ and $\mathrm{y}$ position on target $<2.0$

4. $0.57<$ Beam width in $\mathrm{x}$ and $\mathrm{y}<1.58$

5. Time delay between NuMI time stamp and spill $<0.5 \mathrm{~ns}$

6. Fraction of hits occurring outside the beam peak $<0.45$

7. Number of missing DCMs $=0$

Quality cuts ensure that slices have an analyzable track. The cuts are: 
1. $\mathrm{N}_{\text {hit }}>20$ : Events with less than 20 hits are more likely to be due to neutral-current (NC) interactions, or spurious groupings of noise hits. These events are often not well reconstructed and are removed. Coincidentally this will remove events less than about $0.4 \mathrm{GeV}$ in energy.

2. nkalman $>0$ : This cut requires that the number of 3D Kalman track should be greater than zero, which ensures that we have at least one reconstructed track.

3. Number of contiguous planes in slice $>4$ : Slices that cross less than 4 planes have large reconstruction uncertainties.

\subsubsection{Initial Fiducial and Containment Cut}

The initial shower containment criteria are the same that were used for the 2018 disappearance analysis [99]): the shower start and end points are at least $20 \mathrm{~cm}$ away from the detector edges in $(x, y)$, and in the $\mathrm{Z}$ dimension (along the beam direction) showers are required to have a start and stop position $>20 \mathrm{~cm}$ and $<1525 \mathrm{~cm}$.

\subsubsection{Muon Identification}
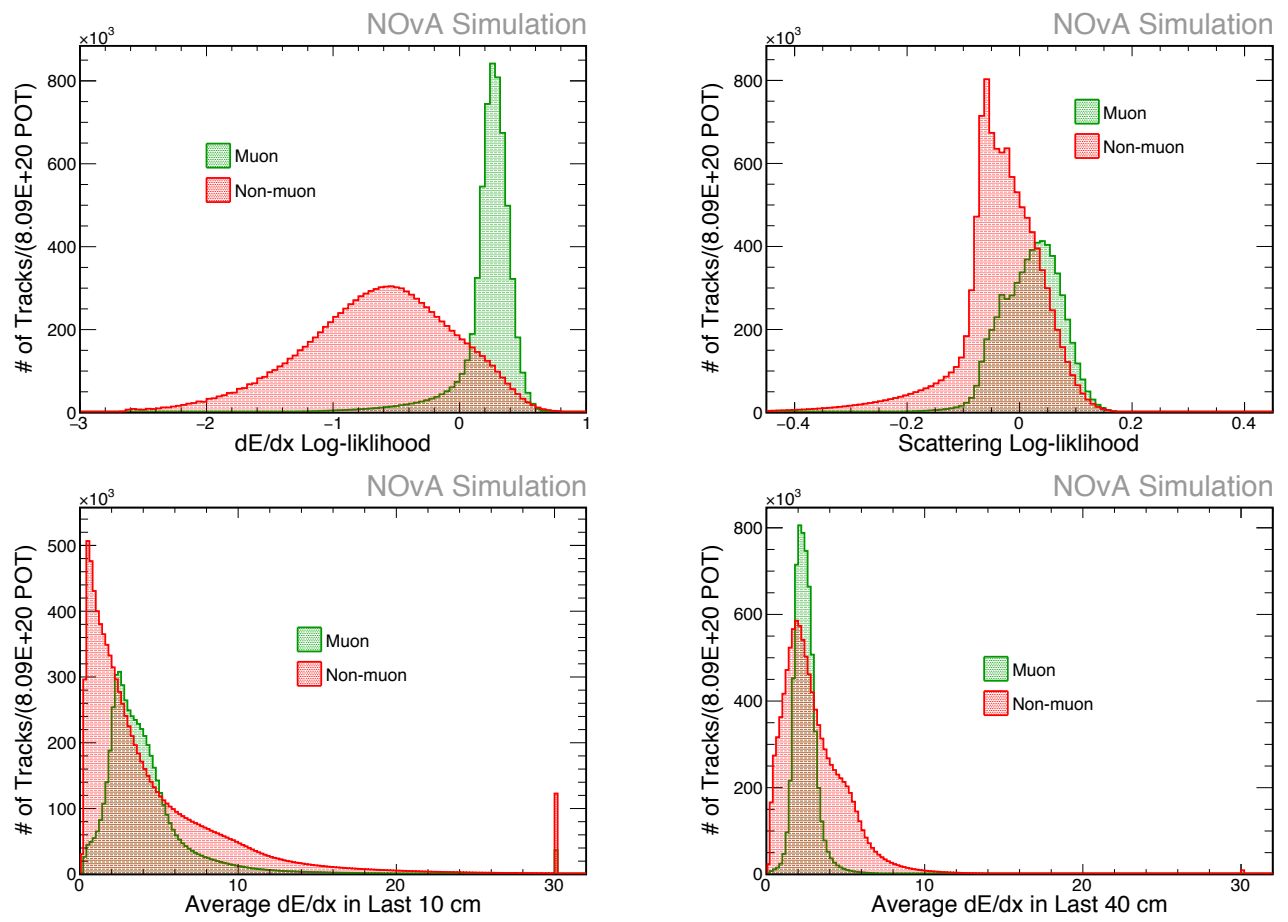

Figure 6.6: Muon and non-muon tracks distributions of $\mathrm{dE} / \mathrm{dx}$ log-likelihood (top left), multiple scattering log-likelihoods (top right), average $\mathrm{dE} / \mathrm{dx}$ in last $10 \mathrm{~cm}$ (bottom left) and average $\mathrm{dE} / \mathrm{dx}$ in last $40 \mathrm{~cm}$ (bottom right) used in the MuonID selector. MC are normalized to data POT $(8.09 \mathrm{e} 20)$.

The signature of a $\nu_{\mu} \mathrm{CC}$ interaction is the presence of a muon in the final state. We have developed a new multivariate muon identification algorithm based on the $\mathrm{dE} / \mathrm{dx}$ log-likelihood difference between that of a muon and a pion (from the ReMId algorithm [92]), the scattering log-likelihood difference between that of a muon and a pion, the average $\mathrm{dE} / \mathrm{dx}$ in the last $10 \mathrm{~cm}$ of the track 

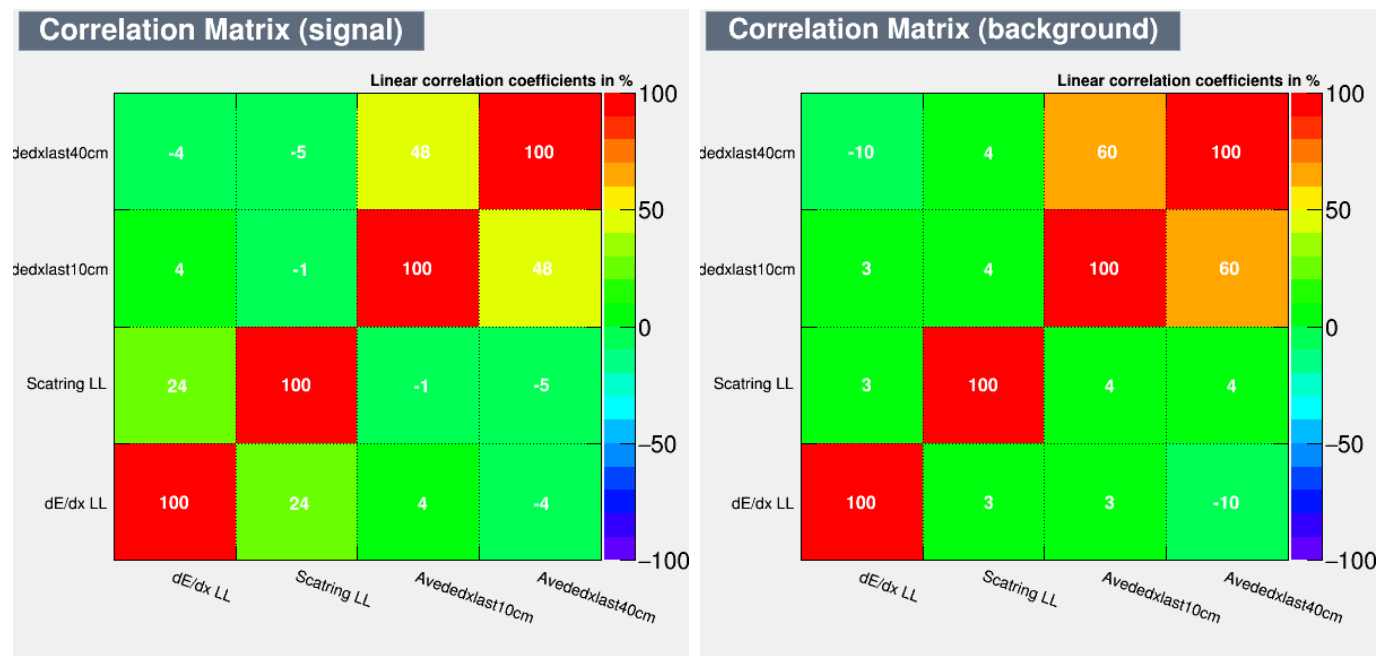

Figure 6.7: Correlation matrix of MuonID input variables for signal tracks (left) and correlation matrix of input variables for background tracks (right)

trajectory, and average $\mathrm{dE} / \mathrm{dx}$ in the last $40 \mathrm{~cm}$ of the track trajectory (see Figure 6.6). These reconstructed variables are input to a Boosted Decision Tree (BDT) algorithm, trained on true muon tracks and background non-muon tracks using reconstructed simulated neutrino interactions that have passed the preselection criteria described above. Additionally, since several of these variables are calculated in the ReMId algorithm, tracks are required to have valid ReMId values (eg, $>-5$ ). Furthermore, there are outliers in the average $\mathrm{dE} / \mathrm{dx}$ distributions with values greater than $30 \mathrm{MeV} / \mathrm{cm}$ due to reconstruction failures; for these tracks, the average $\mathrm{dE} / \mathrm{dx}$ is set to $30 \mathrm{MeV} / \mathrm{c}$. $30 \%$ of the simulation dataset are used in the training sample and tracks are equally divided into training and testing. The output of the BDT is a muon score called MuonID. The correlation matrix of the four input variables for signal and background tracks are shown in Figure 6.7. The distribution of the highest MuonID value for each event passing the preselection in data and MC is shown in Fig. 6.8. The signal event selection efficiency and the fraction of background relative to the number of selected events are shown in Fig. 6.9.

\subsubsection{MuonID Cut Optimization}

The top plots and bottom left plot of Fig. 6.10 show the contributions of the systematic uncertainty on the background prediction, the statistical uncertainty of that prediction, and the systematic uncertainty on the selection efficiency as a function of MuonID cut. The fractional uncertainty on the total cross-section as a function of MuonID cut is shown in the bottom right. From these plots, we see that at low MuonID cut values, the dominant uncertainty is from the predicted background, whereas as higher cut values the uncertainty in the efficiency dominates. The statistical uncertainty on the background prediction is negligible. The optimal cut is Muon ID $>0.34$ for which the fractional uncertainty on the total cross-section has a minimal value of about $3.5 \%$. 


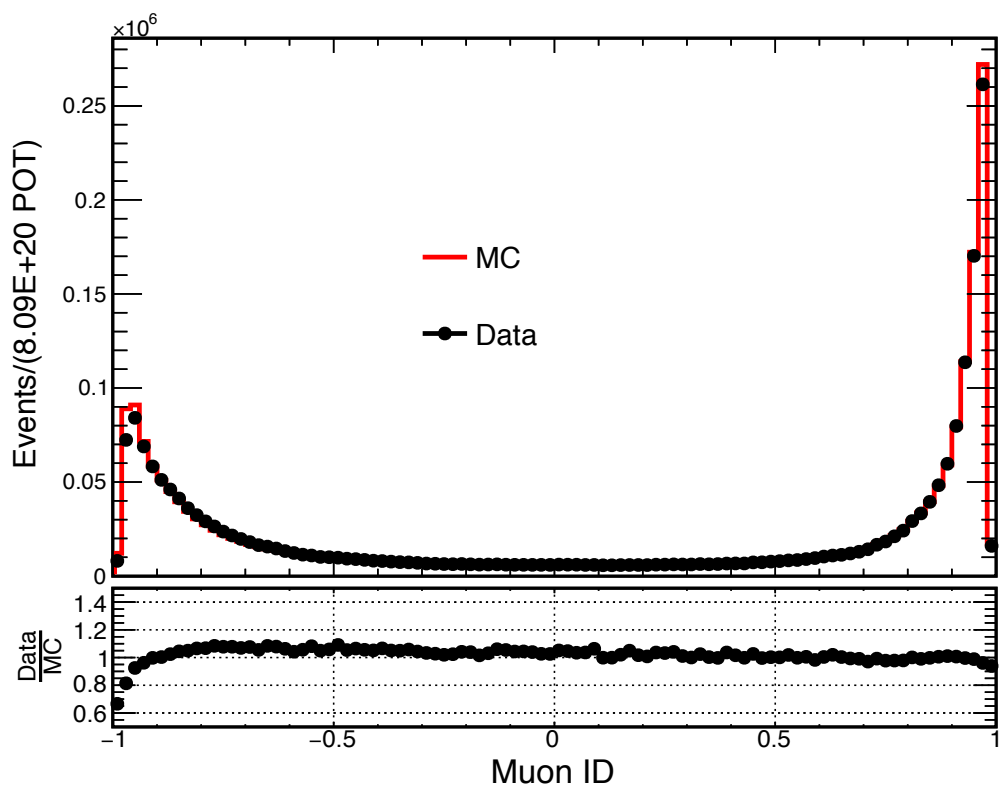

Figure 6.8: ND Data and MC distributions of the highest MuonID value in each event passing preselection. MC distribution is POT-normalized to match the data (8.09e20 POT).
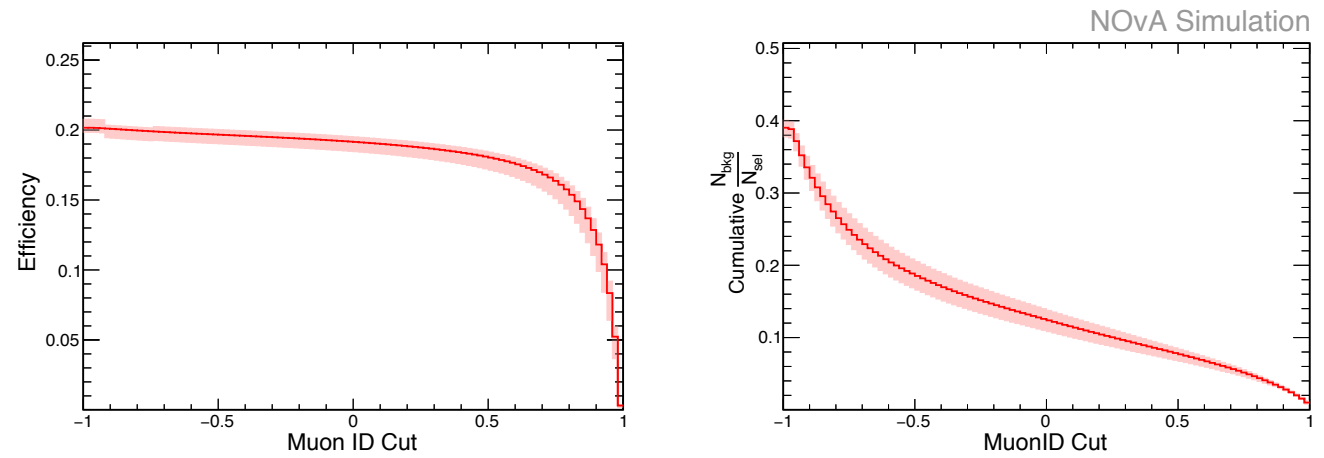

Figure 6.9: Selection efficiency (left) and fraction of background to total number of events selected (right) as a function of MuonID cut value. 

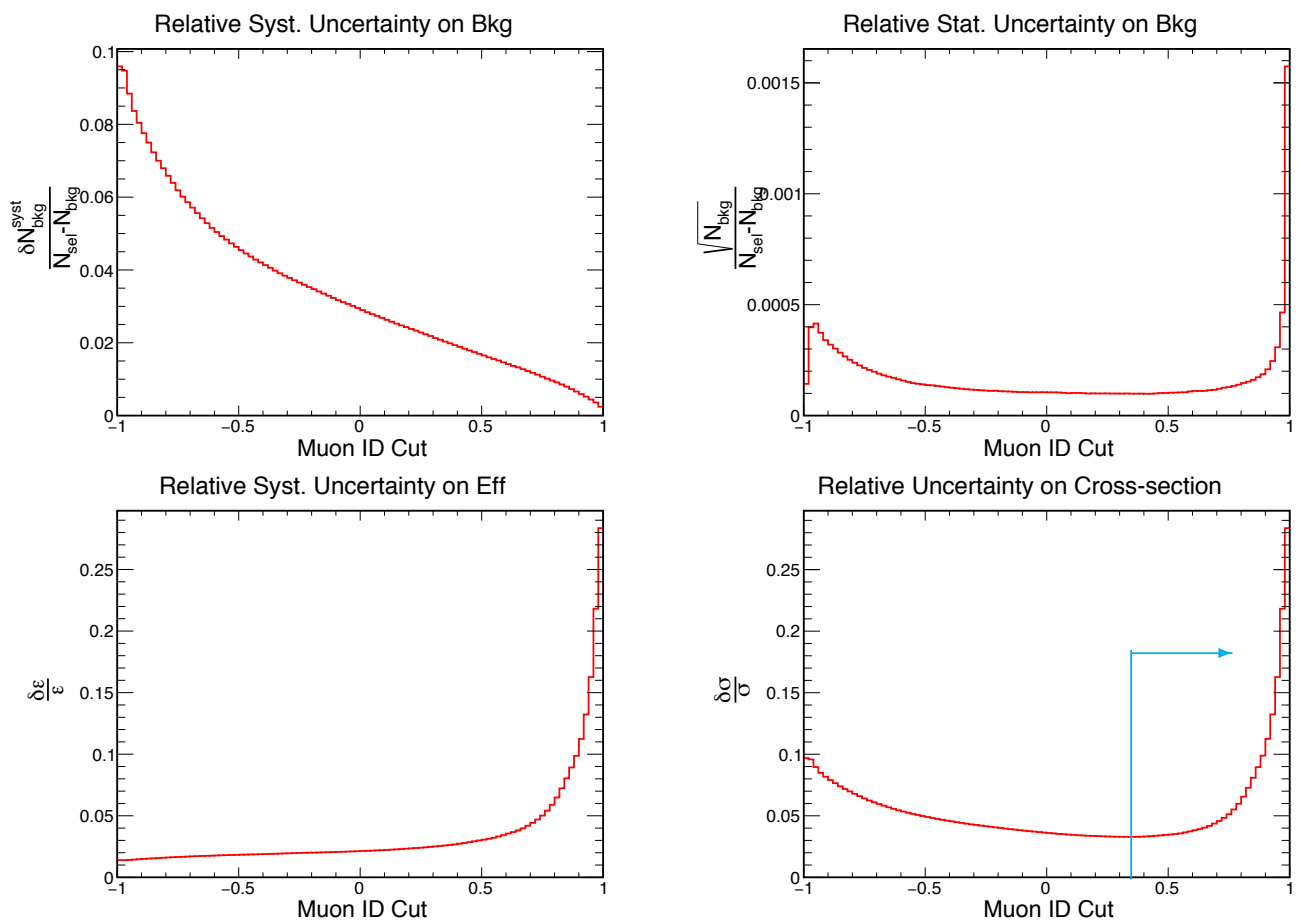

Figure 6.10: Each bin is filled with total uncertainty if the chosen cut was the left edge of that bin. Muon ID $>0.34$ are retained as candidate of $\nu_{\mu}$ CC events.
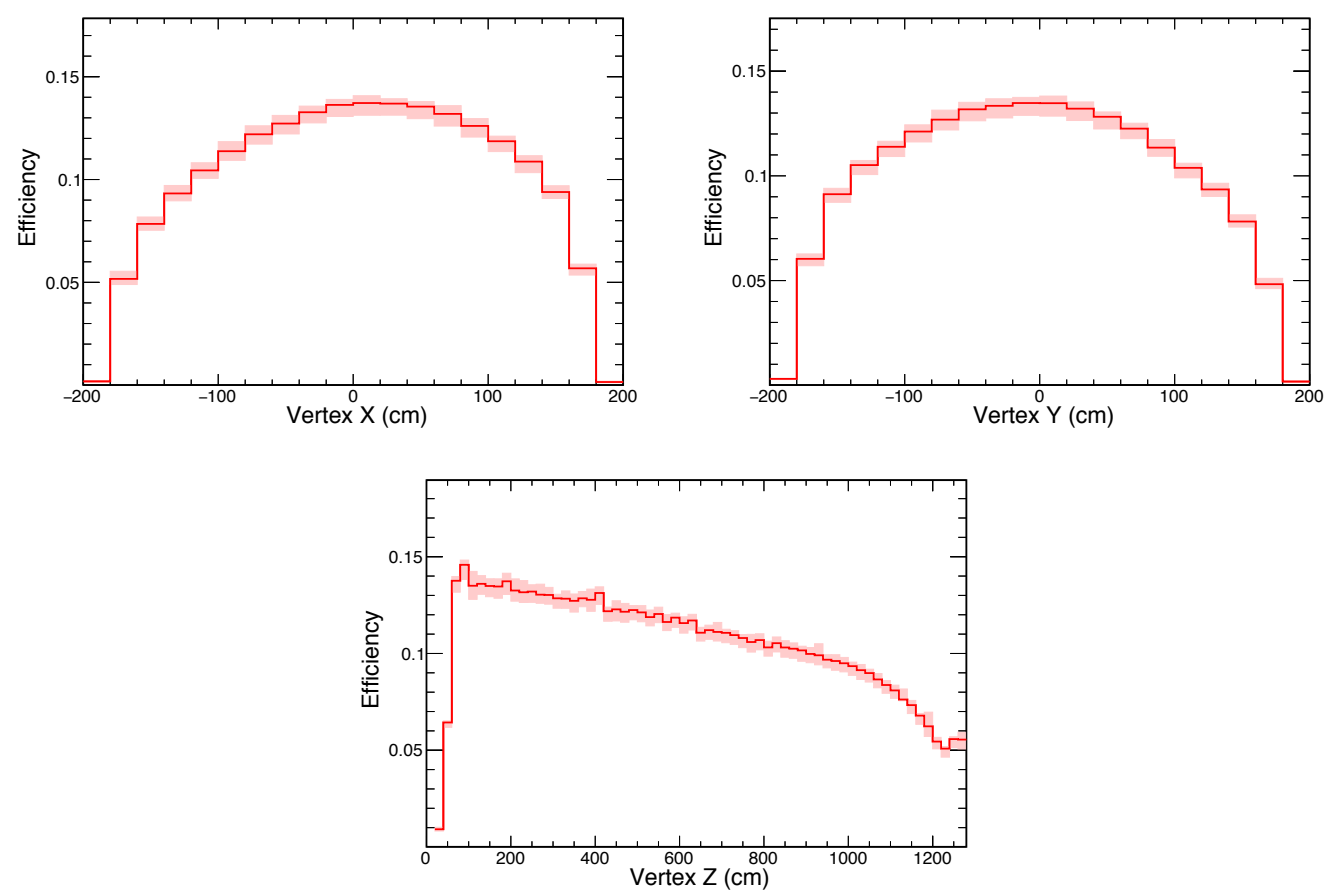

Figure 6.11: Efficiency distribution as a function of vertex $\mathrm{x}$ (left), vertex y (middle) and vertex $\mathrm{z}$ (right) with systematic error. 

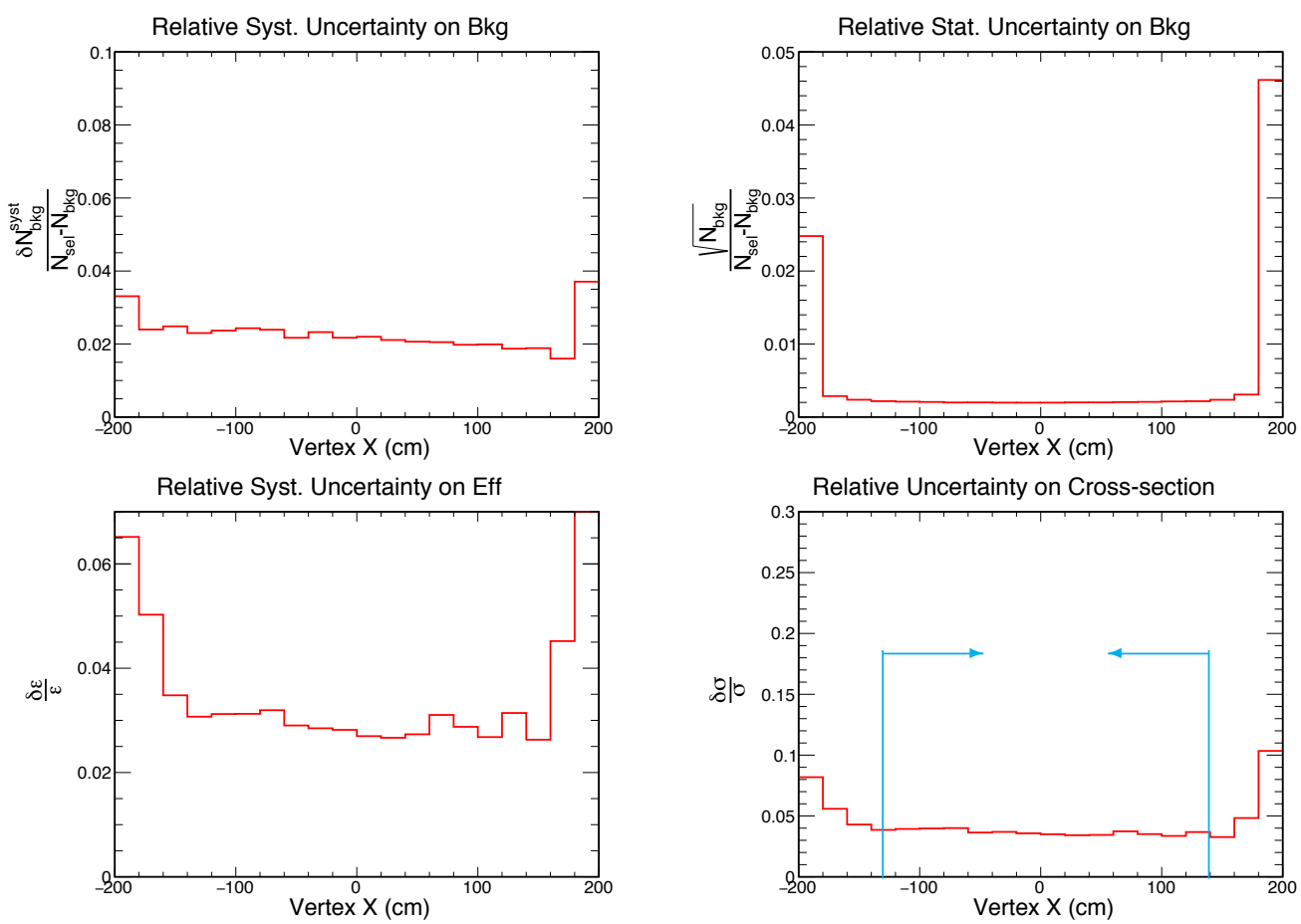

Figure 6.12: $-130<$ vertex $\mathrm{X}(\mathrm{cm})<140$, cyan color lines are drawn where we see (by eye ball) uncertainties beginning to increase.
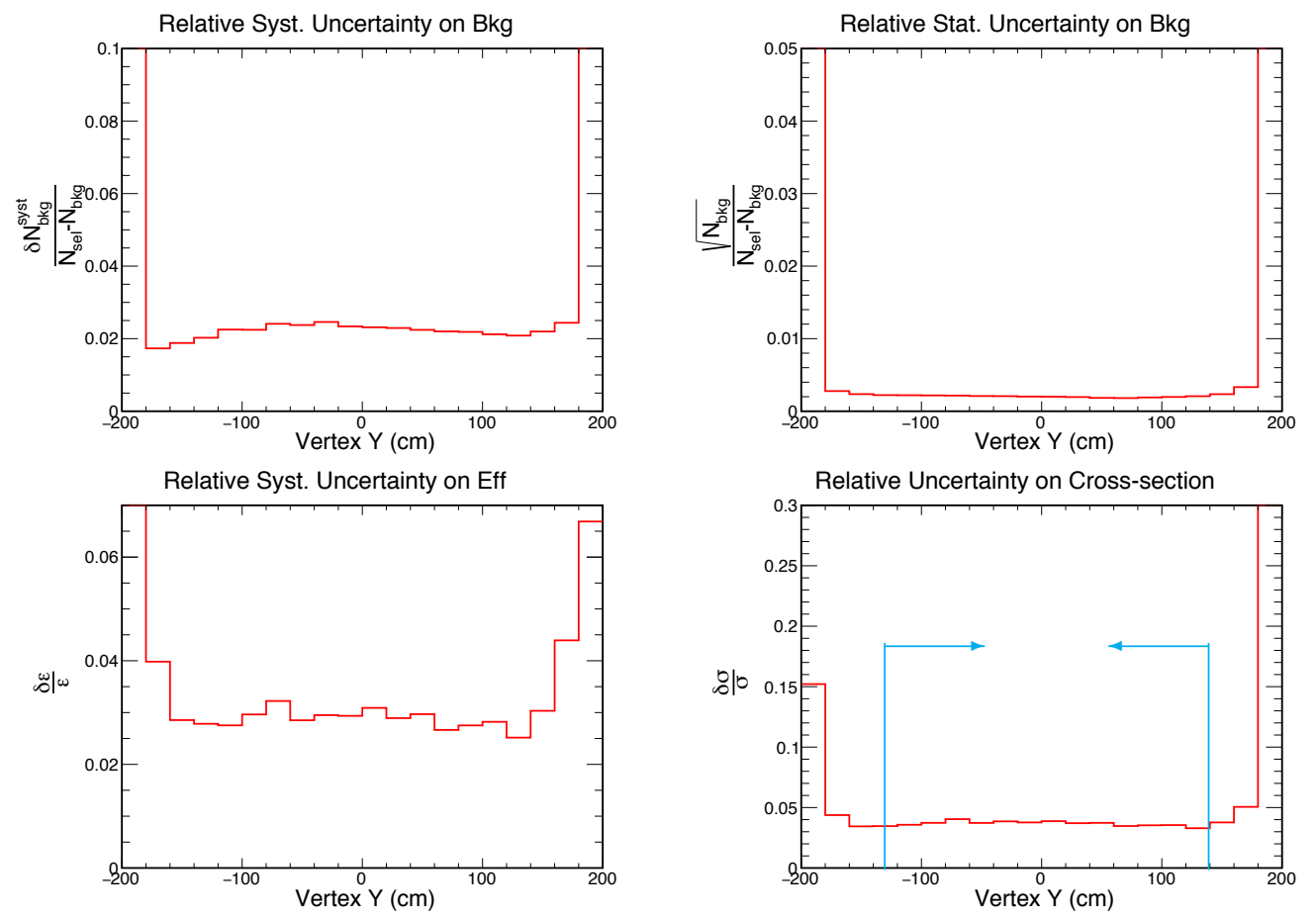

Figure 6.13: $-130<$ vertex Y $(\mathrm{cm})<140$, cyan color lines are drawn where we see (by eye ball) uncertainties beginning to increase. 

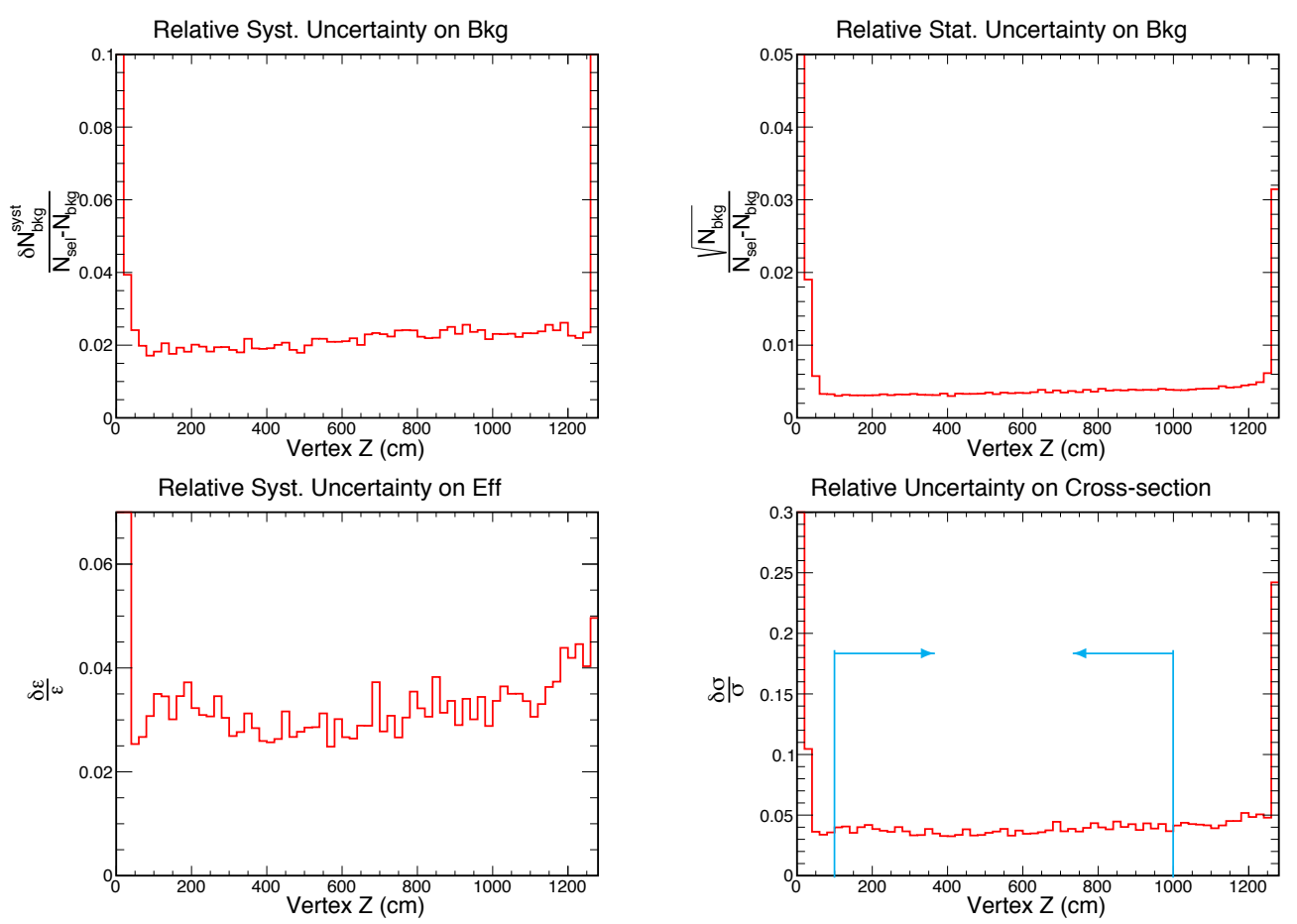

Figure 6.14: $100<$ vertex Z $(\mathrm{cm})<1000$, cyan color lines are drawn where we see (by eye ball) uncertainties beginning to increase.

\subsubsection{Optimization of Fiducial Volume and Containment Criteria}

With the muon identification criteria determined, we next apply further event selection criteria based on the selected muon track. The following cuts are similar to that used by the $\nu_{\mu} \mathrm{CC}$ disappearance analysis, with the only difference being that the track with the highest MuonID score is used instead of the track with the highest ReMId score:

1. The forward projection of the muon candidate track is greater than 5 cells from the edge of the detector. This is aimed at removing events with uncontained muons.

2. The backward projection of the muon candidate track is greater than 10 cells from the edge of the detector. This is aimed at removing contamination from neutrino interactions outside the detector.

3. Remove all events with muon candidate tracks that could have exited the active region of the detector before re-entering into the muon catcher ("air gap events"): the end z-position of the track is less than $1275 \mathrm{~cm}$ or the

(trk.kalman.tracks[ibesttrk].stop.Z ()$<1275 \|$ trk.kalman.tracks[ibesttrk].trkyposattrans $<$ $55)$.

We optimize the fiducial volume for this analysis by studying the fractional uncertainty on the total cross-section as a function of reconstructed vertex position. The previously defined preselection and track containment cuts result in a drop in selection efficiency (see Fig. 6.11) and a corresponding drop in selected backgrounds near the edges of the detector. However, what matters is how well these 
drops are modeled in the simulation. Figures 6.12, 6.13 and 6.14 show the fractional uncertainty of the total cross-section, and the main contributions to the uncertainty, as a function of reconstructed vertex $(x, y, z)$ for events passing the preselection. In this study, the fiducial volume used to define the signal is a box with the following $(x, y, z)$ bounds that reflect the physical boundaries of the detector:

- $-191<\mathrm{X}(\mathrm{cm})<192$

$\bullet-187<\mathrm{Y}(\mathrm{cm})<194$

- $0<\mathrm{Z}(\mathrm{cm})<1270$

We find that the cross-section uncertainty is fairly flat for the fiducial volume defined by the following reconstructed vertex position bounds:

1. $-130<\mathrm{X}(\mathrm{cm})<140$,

2. $-130<\mathrm{Y}(\mathrm{cm})<140$,

3. $100<\mathrm{Z}(\mathrm{cm})<1000$

Cyan arrows in Figs. 6.12-6.14 are drawn to indicate the location of the cuts.

As a cross-check, we compare the vertex (track start) and track end positions between data and simulation with all but the containment cut applied, and again with all cuts applied. Figures 6.15 and 6.16 show that the shapes of these distributions agree to within a few percent overall.
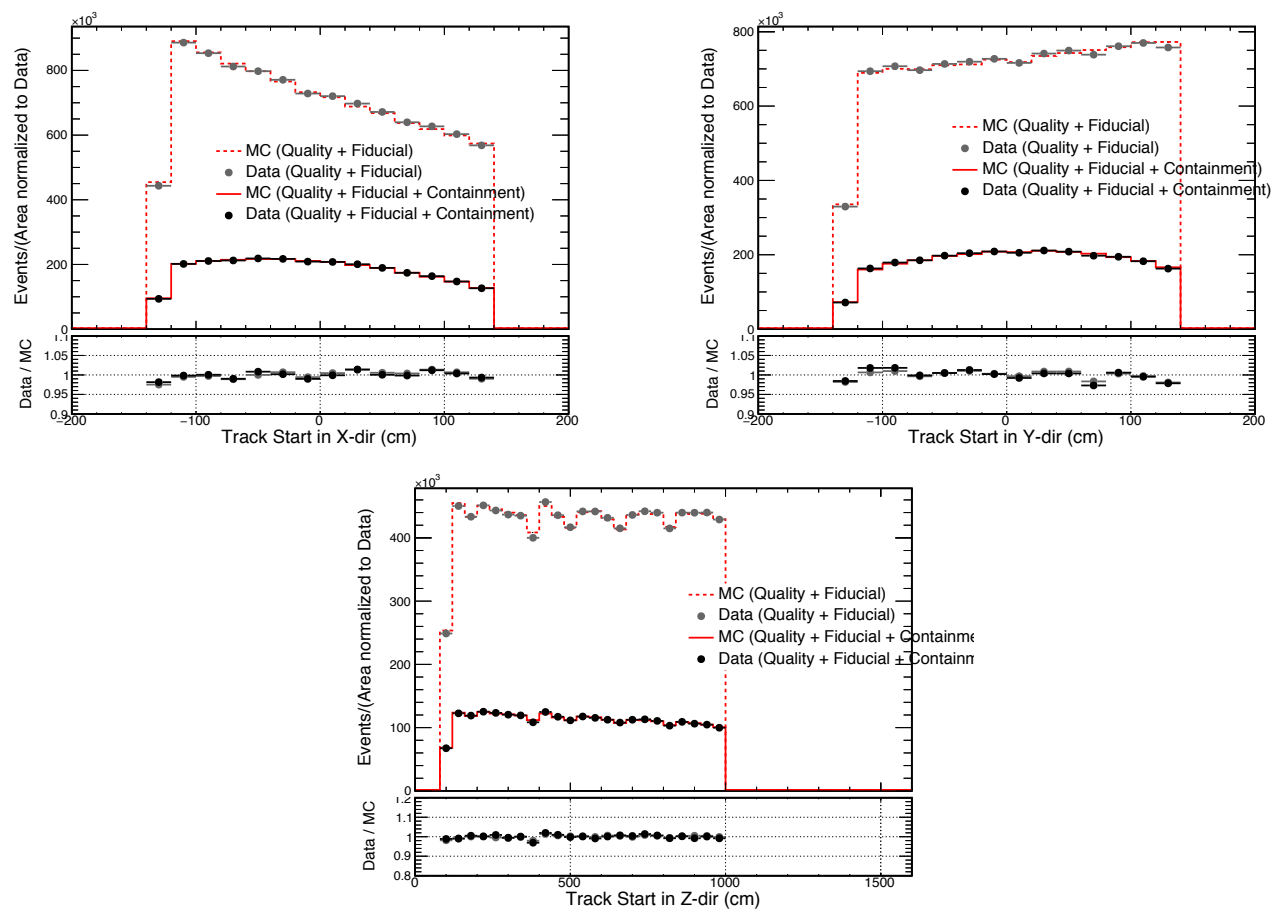

Figure 6.15: Data-to-MC comparison of the reconstructed vertex positions before and after containment cuts are applied. Data/MC ratios are drawn below the distributions. MC are area normalized to match the data. Preselection, MuonID and fiducial cuts are applied. 

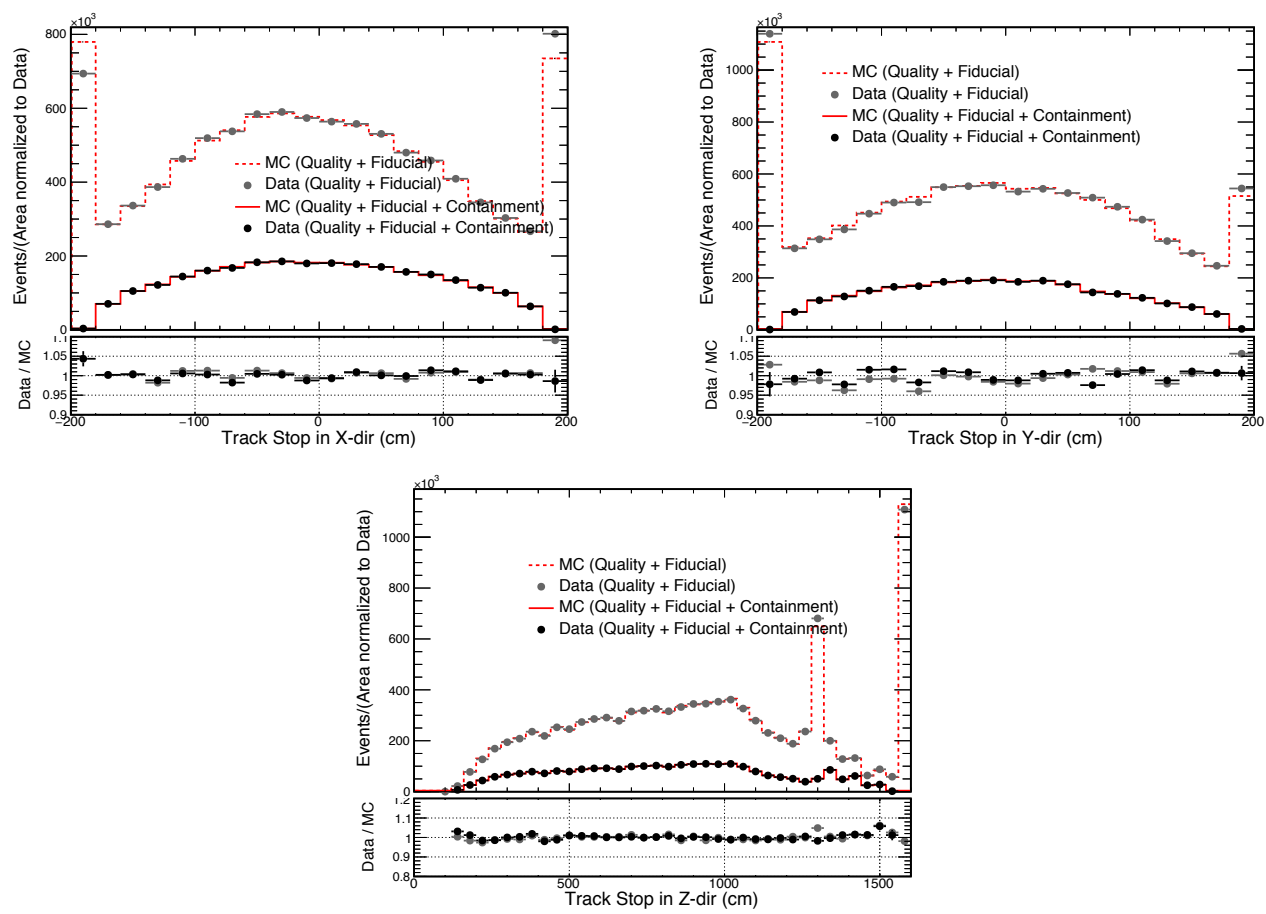

Figure 6.16: Data-to-MC comparison of the reconstructed track end point positions before and after containment cuts are applied. Data/MC ratios are drawn below the distributions. MC are area normalized to match the data. Preselection, MuonID and fiducial cuts are applied.

\subsubsection{Event Selection Cut Flow Tables}

Table 6.1 is the cut flow table where cuts listed in the first column are sequentially applied and Table 6.2 in the "N-1" table where for each row, all cuts have been applied, other than the one names on that row. These tables summarizes the impact of the selection criteria on the MC events. We note too that a comparison of the performance in terms of efficiency and background rejection between ReMId and MuonID can be found in [100].

\begin{tabular}{llllll}
\hline Cut & $\nu_{\mu}$ & $\bar{\nu}_{\mu}$ & $\mathrm{NC}$ & $\nu_{e}+\bar{\nu}_{e}$ & Non-fiducial \\
\hline Slicing & $7.91 \mathrm{e}+06(5.58)$ & $310268(0.22)$ & $2.33 \mathrm{e}+06(1.64)$ & $172036(0.12)$ & $1.31 \mathrm{e}+08(92.43)$ \\
quality & $7.82 \mathrm{e}+06(7.96)$ & $305971(0.31)$ & $1.73 \mathrm{e}+06(1.76)$ & $170619(0.17)$ & $8.82 \mathrm{e}+07(89.79)$ \\
Showerfid\&cont & $2.24 \mathrm{e}+06(24.9)$ & $71161.6(0.79)$ & $902224(10.1)$ & $78509.1(0.87)$ & $5.68 \mathrm{e}+06(63.3)$ \\
MuonID & $1.68 \mathrm{e}+06(37.1)$ & $60189.7(1.33)$ & $128814(2.84)$ & $8777.88(0.19)$ & $2.66 \mathrm{e}+06(58.57)$ \\
Trackfid\&cont & $1.18 \mathrm{e}+06(86.4)$ & $35236.8(2.57)$ & $104127(7.60)$ & $6006.92(0.44)$ & $40544.4(2.96)$ \\
\hline
\end{tabular}

Table 6.1: Impact of each selection cut on the total fraction of simulated signal and background events. The numbers in the parentheses indicate the percentage a particular event category contributes to the total at that cut level. True fiducial cut have been applied throughout. 


\begin{tabular}{llllll}
\hline Cut(N-1) & $\nu_{\mu}$ & $\bar{\nu}_{\mu}$ & $\mathrm{NC}$ & $\nu_{e}+\bar{\nu}_{e}$ & Non-fiducial \\
\hline All & $1.18 \mathrm{e}+06(86.4)$ & $35200.2(2.57)$ & $104119(7.61)$ & $6005.88(0.44)$ & $40437.6(2.96)$ \\
Quality & $1.19 \mathrm{e}+06(85.7)$ & $35861.4(2.59)$ & $109328(7.89)$ & $6015.99(0.43)$ & $47086.6(3.40)$ \\
Showerfid\&cont & $1.63 \mathrm{e}+06(79.5)$ & $46255.4(2.25)$ & $184848(9.01)$ & $14043.4(0.68)$ & $174285(8.50)$ \\
MuonID & $1.49 \mathrm{e}+06(58.2)$ & $38285(1.49)$ & $715011(27.9)$ & $58932.5(2.30)$ & $258704(10.1)$ \\
Trackfid\&cont & $1.68 \mathrm{e}+06(37.1)$ & $60088.7(1.33)$ & $128754(2.85)$ & $8768.19(0.19)$ & $2.64 \mathrm{e}+06(58.5)$ \\
\hline
\end{tabular}

Table 6.2: N-1 cut table shows the impact of each selection cut on the total fraction of simulated signal and background events. The numbers in the parentheses indicate the percentage a particular event category contributes to the total at that cut level. True fiducial cut have been applied throughout. The first row, labelled "All" has all cuts applied. For all other row, all selection criteria, but the one named on that row have been applied.

\begin{tabular}{llllll}
\hline Cut & QE $(\%)$ & Res (\%) & DIS (\%) & Coh (\%) & MEC (\%) \\
\hline Slicing & 17.82 & 27.79 & 43.17 & 0.71 & 10.51 \\
Quality & 17.63 & 27.70 & 43.57 & 0.72 & 10.38 \\
Showerfid\&cont & 20.15 & 35.46 & 27.09 & 1.35 & 15.95 \\
MuonID & 21.91 & 35.59 & 22.5 & 1.56 & 18.43 \\
Trackfid\&cont & 20.85 & 38.68 & 19.80 & 1.79 & 18.88 \\
\hline
\end{tabular}

Table 6.3: Impact of each selection cut on the the signal events broken down by interaction type. Each row indicates the sample composition in percentage by mode at the cut level specified in the first column.

\subsection{Muon and Neutrino Energy Estimation, Resolution and Binning}

The muon and muon-neutrino energy estimators developed for this analysis are similar to the one used in the disappearance oscillation analysis[101] with some changes that are discussed below as well as in $[102,103]$. Simulated neutrino interactions are used to relate reconstructed track and slice observables to the energy of the neutrino. The general procedure is to first determine the muon energy by relating the track length to the true energy of the muon. Next, the calorimetric energy in the slice not associated with the muon is associated to the hadronic energy in the event. This is done by requiring the summed reconstructed muon and hadronic energies to be that of the true neutrino energy.

\subsubsection{Muon Energy}

In our muon energy estimator, we divide tracks into categories: those that are fully contained in the fully active region, and those that stop in the muon catcher. For muons which are fully contained in the active region, the distribution of true energy in each bin of reconstructed track length is fit with a Gaussian to obtain the mean and variance. A polynomial of degree 3 is fitted to the mean of truncated true muon energy as a function of the track length (top left of Fig. 6.17 and Fig.6.18). For muons that start in the active region and stop in the muon catcher, a similar procedure is followed however the track length is divided into two parts: track length in the active region plus track length in the muon catcher. A polynomial of degree 2 is used in the fit for the active region (top right of 
Fig. 6.17 and Fig. 6.18), and a straight line is used in the fit for the muon catcher (bottom left of Fig. 6.17 and Fig. 6.18).

\subsubsection{Hadronic Energy}

The reconstructed visible hadronic energy is defined as the sum of calorimetric energy of hits in the slice that is not associated with the muon track, and any additional energy that may be deposited by hadrons on and near the start of the muon track. The latter is reconstructed by subtracting MIP energy from the first few planes of the muon track[104]. The reconstructed muon energy is subtracted from the true neutrino energy to get a measure of the leftover energy, which is then fitted to a polynomial of degree 4 as a function of reconstructed visible hadronic energy (bottom right of Fig. 6.17 and Fig. 6.18).

The reconstructed neutrino energy is defined as the sum of the reconstructed muon energy and the reconstructed hadronic energy.

$$
E_{\nu}=E_{\mu}+E_{h a d}
$$

A comparison of the true and reconstructed distributions are shown in Fig. 6.19, and Fig. 6.20 shows the distributions of the fractional difference between true and reconstructed observables.

\subsubsection{Optimization of Polynomial Fitting}

The fit functions used in the muon and hadronic energy estimators are polynomials. The fit $\chi^{2} /$ dof can be improved slightly using higher order polynomials, however, the additional terms in the polynomial are often sensitive to statistical fluctuations. Instead, we use the absolute resolution as the metric for deciding on the order of the polynomial, keeping the function as simple as possible.

Figure 6.21 shows the absolute resolution of the muon energy estimators and hadronic energy estimator as a function of the true observable, for different orders of the polynomial fit function. The pol3 is chosen as the best function for muons starting and stopping in the active region, the pol2 for the muon energies deposited in the active region for muons stopping in the muon catcher, and a straight line for the muon energies deposited in the muon catcher for muons stopping in the muon catcher. A pol4 is used for the hadronic energy.

Figure 6.22 shows the bias (mean and error on the mean of reconstructed - true value) plot for each case of muon energy estimation and hadronic energy estimation. From this figure, we conclude that there is no significant change in the bias for the different polynomial fits.

\subsubsection{Resolution and Binning}

The binning of the muon kinetic energy $\left(T_{\mu}\right)$ and angle $\left(\cos \theta_{\mu}\right)$ for this analysis are based on several considerations: resolution, statistics, and practicality of having a reasonable number of bins. All bins are at least as wide as the estimated resolution. Low statistics is a consideration for some of the more extreme regions of phase space.

The resolution is determined from the distribution widths of the difference between reconstructed and true kinematic variable for selected simulated signal events. Figure 6.23 shows the predicted spectra (red lines) and resolutions (black dots) of $T_{\mu}$ and $\cos \theta_{\mu}$ as a function of their true value. The average $T_{\mu}$ resolution is $50 \mathrm{MeV}$, and the average $\cos \theta_{\mu}$ resolution is 0.04 . As a result of this study, the following binning scheme has been adopted: 

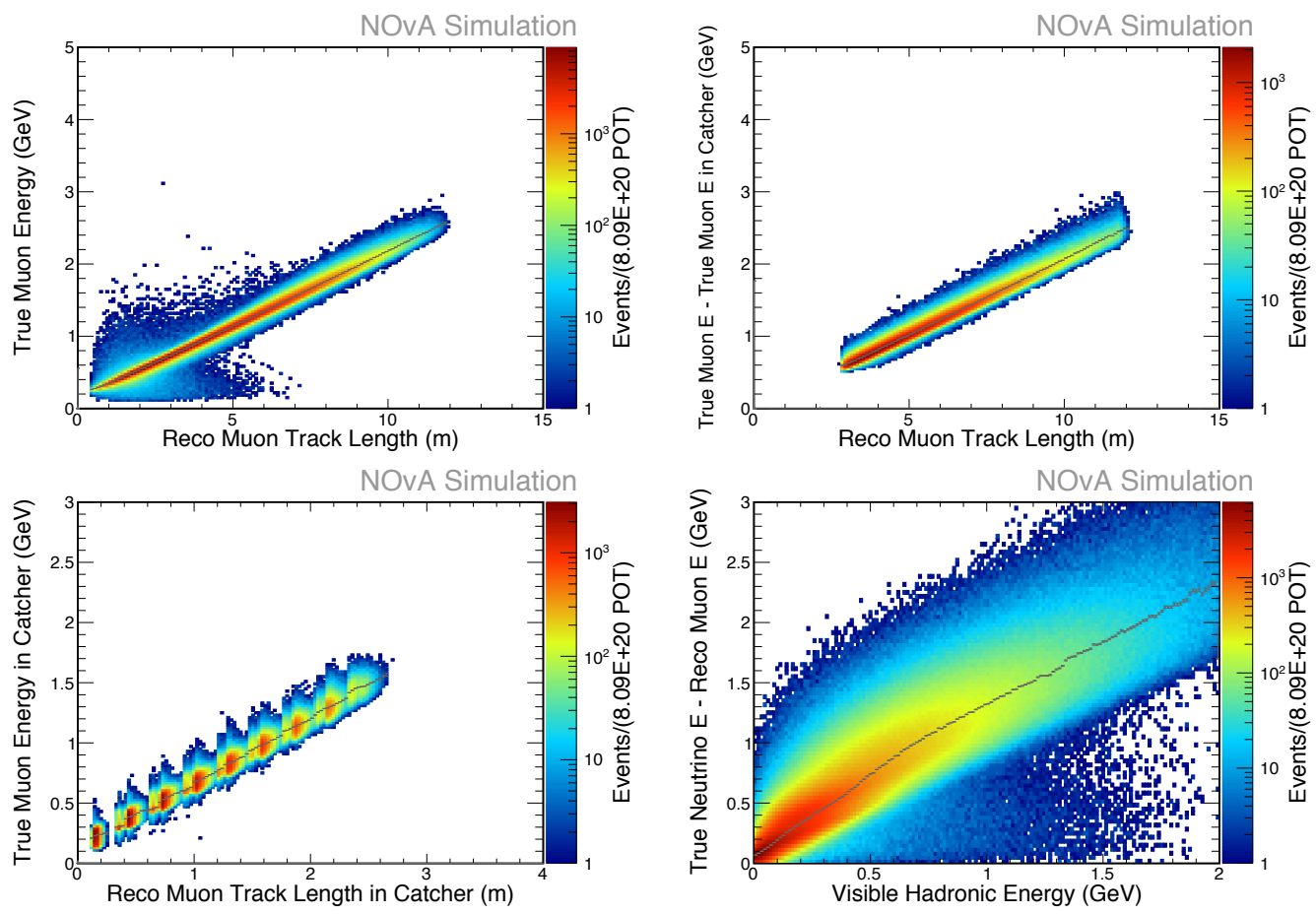

Figure 6.17: Top left: Reconstructed muon track length in meters as a function of the true muon energy in $\mathrm{GeV}$ for the fully active population. Top Right: Reconstructed muon track length in the active region in meters vs. total true muon energy - true muon energy when muon enters the muon catcher for the active and catcher population. The color axis is an arbitrary number of interactions and is displayed logarithmically (top right). Bottom left: Reconstructed muon track length in the muon catcher vs. true muon energy in the muon catcher in GeV. Bottom right: Visible hadronic energy in $\mathrm{GeV}$ vs. true neutrino energy minus reconstructed muon energy in GeV. The horizontal axis has variable binning. The color axis is an arbitrary number of interactions and is display logarithmically (bottom right). 

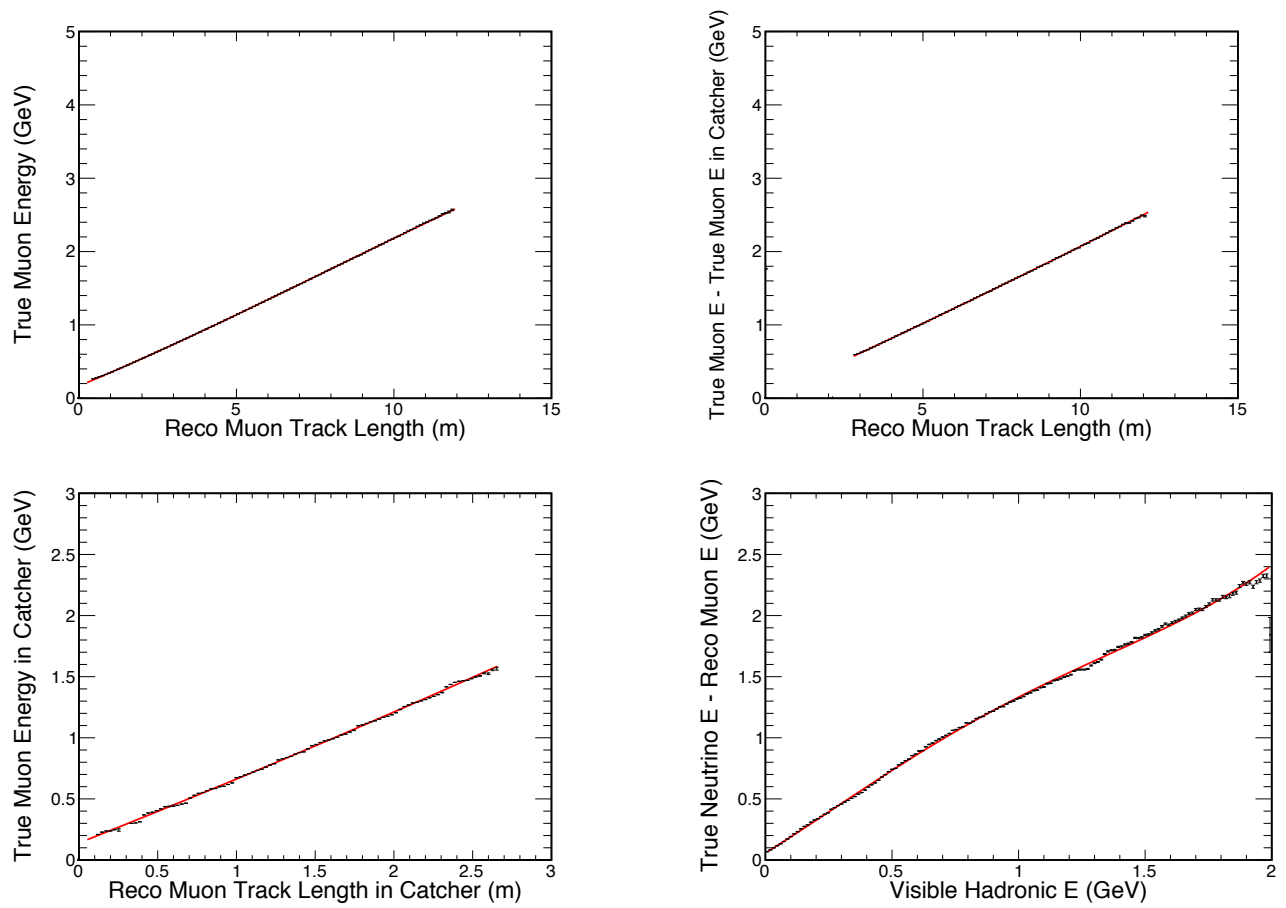

Figure 6.18: Polynomial fit line (order 3) overlaid on graph points used to make the fit. The fit relates reconstructed muon track length in meter to true muon energy in $\mathrm{GeV}$ (top left). The fit relates reconstructed muon track length in meter in the active region to true muon energy in the active region in $\mathrm{GeV}$ (top right). The fit relates reconstructed muon track length in catcher region in meter to true muon energy in catcher region in $\mathrm{GeV}$ (bottom left). The fit relates Visible hadronic energy in $\mathrm{GeV}$ to expected hadronic energy (neutrino energy - reconstructed muon energy) in $\mathrm{GeV}$ (bottom right). 

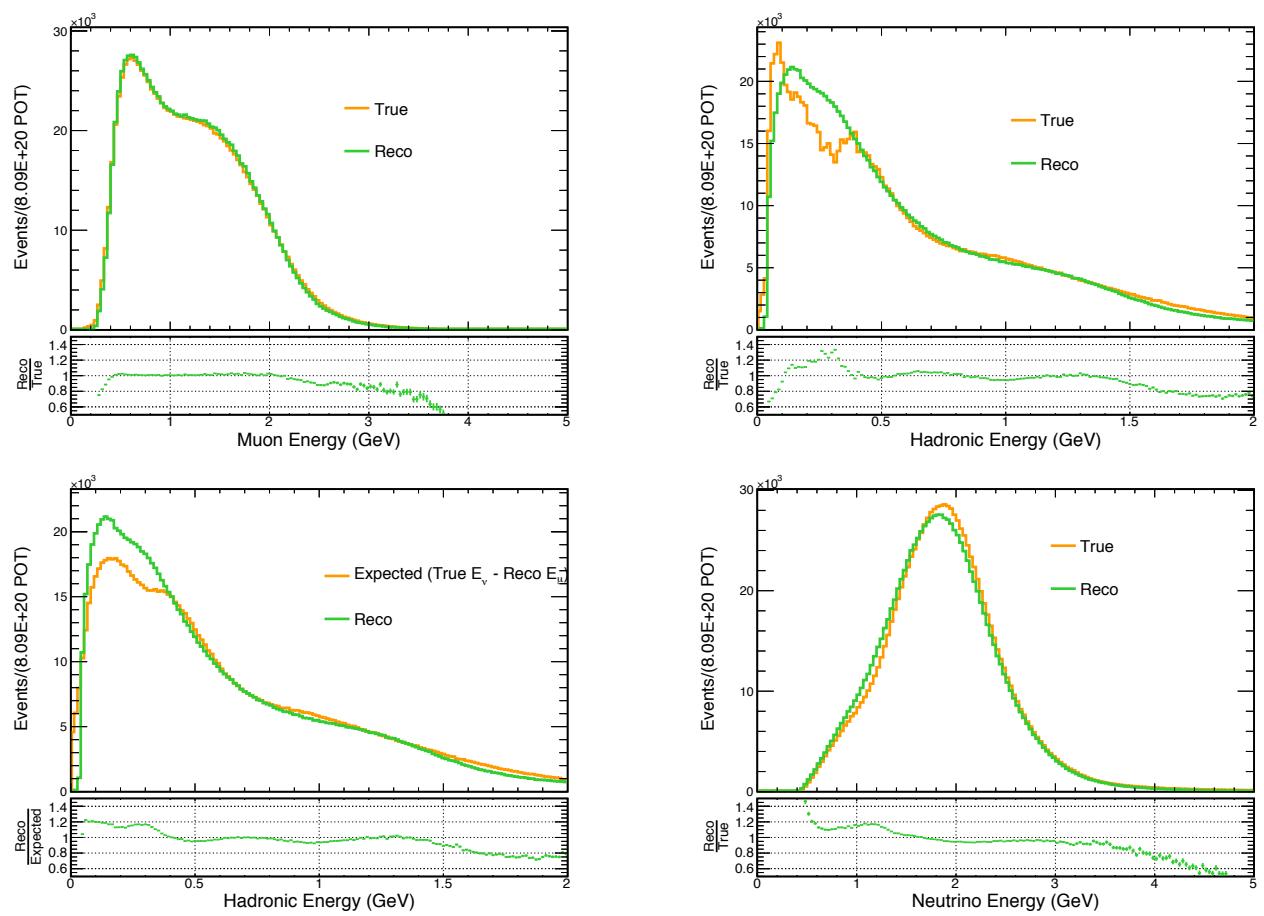

Figure 6.19: Comparison of true and reconstructed distribution on top and bottom plot shows the ratio between true and reconstructed distribution for muon energy (top left), hadronic energy(top right and bottom left) and neutrino energy (bottom right) respectively.

- 20 equal-sized bins from $0.5-2.5 \mathrm{GeV}$ for reconstructed $T_{\mu}$ and

- 13 variable-sized bins for reconstructed $\cos \theta_{\mu}$ : 5 bins between $0.5-0.8,1$ bin between $0.8-0.85$, 3 bins between 0.85-0.94, 2 bins between 0.94-0.98, and 2 bins between $0.98-1.0$.

Figure 6.24 shows the predicted number of selected events in each bin for an exposure of $8.09 \mathrm{e} 20$ POT.

\subsubsection{Muon Kinematic Cuts}

Because of the limited acceptance of the near detector, we constrain this measurement to the region of kinematic phase space of the final-state muon that has reasonable uncertainties on the measurement. Fig. 6.25 shows the rates and their uncertainties of the predicted wrong-sign-background and the non- $\nu_{\mu}$ CC-like interactions in the detector as a function of the reconstructed muon energy and angle. Fig. 6.26 shows the efficiency (top left), uncertainty on the efficiency (top right), systematic uncertainty on the background (bottom left) and statistical uncertainty on the signal (bottom right) as a function of muon energy and angle. Finally, Fig. 6.27 shows the relative uncertainty in the differential cross-section measurement in each bin. We limit our measurement to fractional uncertainties less than approximately $30 \%$, in particular:

1) $\cos \theta_{\mu}<0.56$ and $T_{\mu}<0.9$ or

2) $0.56 \leq \cos \theta_{\mu}<0.68$ and $T_{\mu}<1.0$ or 

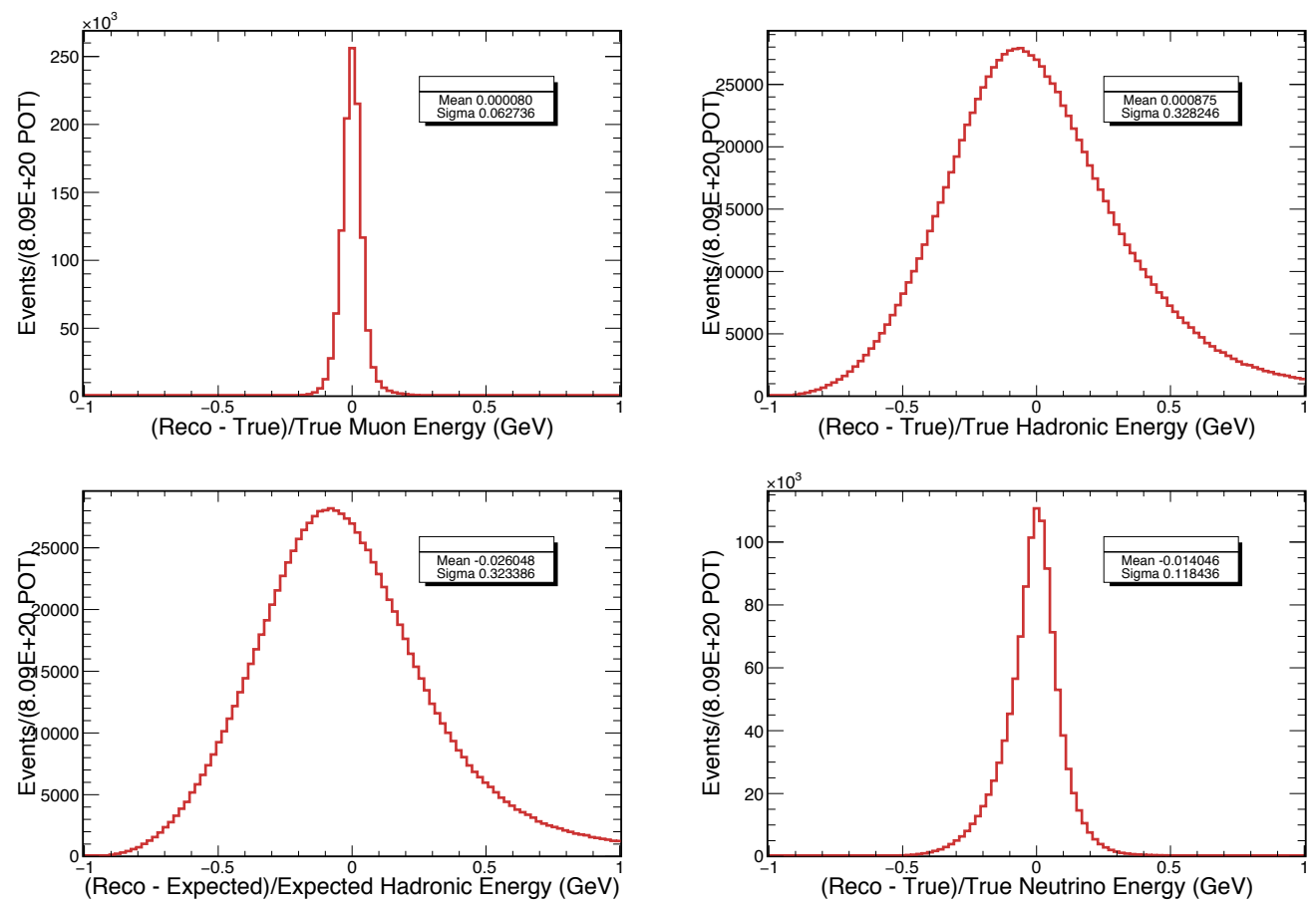

Figure 6.20: Relative resolution for reconstructed muon energy (top left), reconstructed hadronic energy (top right), reconstructed expected hadronic energy (bottom left) and reconstructed neutrino energy respectively (bottom right).

3) $0.68 \leq \cos \theta_{\mu}<0.74$ and $T_{\mu}<1.2$ or

4) $0.74 \leq \cos \theta_{\mu}<0.80$ and $T_{\mu}<1.4$ or

5) $0.80 \leq \cos \theta_{\mu}<0.85$ and $T_{\mu}<1.5$ or

6) $0.85 \leq \cos \theta_{\mu}<0.88$ and $T_{\mu}<1.7$ or

7) $0.88 \leq \cos \theta_{\mu}<0.91$ and $T_{\mu}<2.0$ or

8) $0.91 \leq \cos \theta_{\mu}<0.94$ and $T_{\mu}<2.4$ or

9) $0.94 \geq \cos \theta_{\mu}$

\subsection{Efficiency Correction}

The efficiency correction accounts for the loss of true signal to detector acceptance and the signal selection criteria. It is applied to the background subtracted, unfolded distribution, to recover the estimated true signal events as a function of a quantity of concern.

Figure 6.28 shows the efficiency of true signal selection as a function of true muon kinetic energy, true $\cos \theta_{\mu}$ with respect to mean neutrino direction, true neutrino energy, and true inelasticity. The denominator of the efficiency ratio in these distributions is the true signal, ie true fiducial $\nu_{\mu}$ $\mathrm{CC}$ interactions with muon kinematics in the allowed region. Figure 6.29 shows the efficiency of 

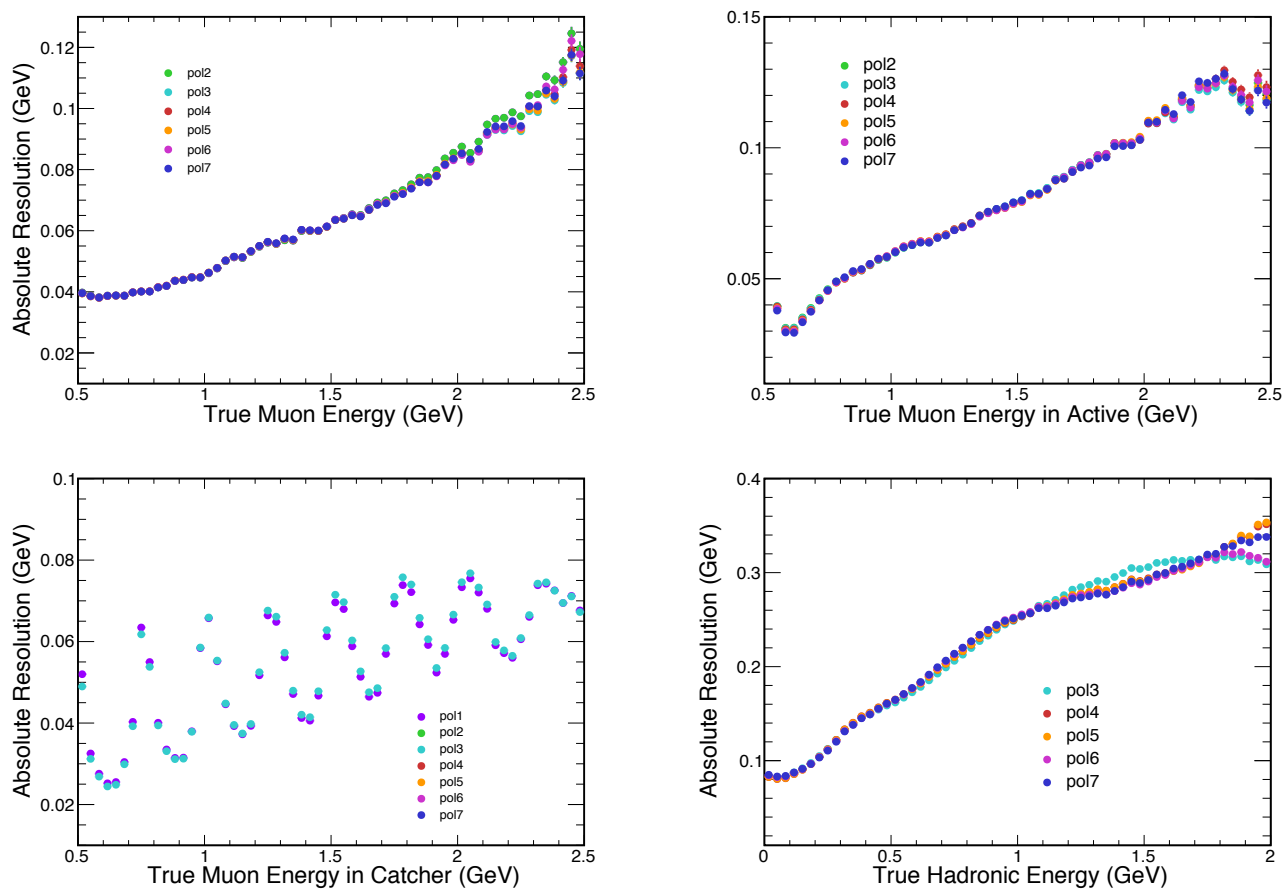

Figure 6.21: Absolute resolution distribution (rms and error on the rms of reconstructed - true distribution) for different polynomial fit as a function of muon energy (top left), muon energy in active region (top right), muon energy in catcher region (bottom left) and hadronic energy (bottom right) respectively. 

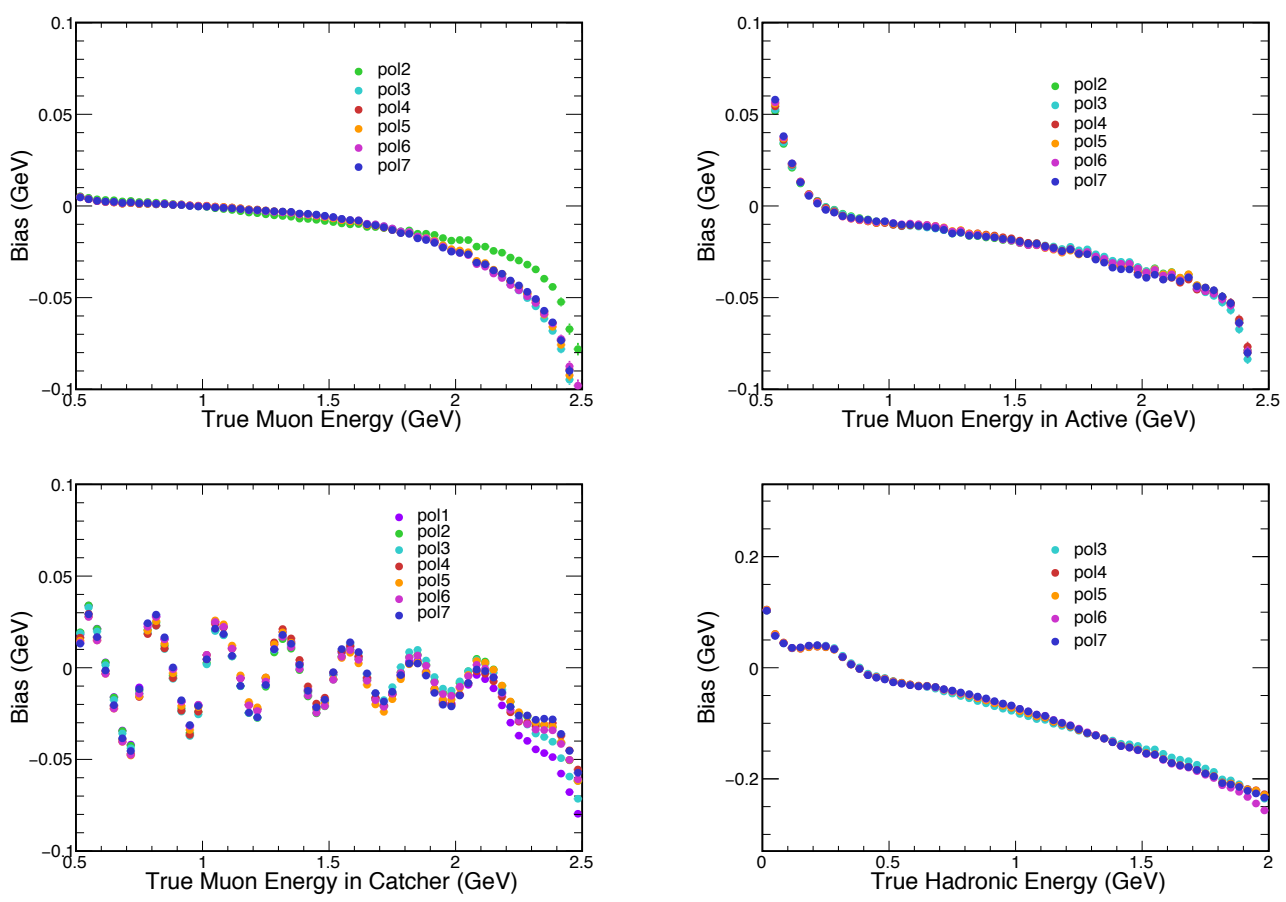

Figure 6.22: Bias distribution (mean and error on the mean of reconstructed - true distribution) for different polynomial fit as a function of muon energy (top left), muon energy in active region (top right), muon energy in catcher region (bottom left) and hadronic energy (bottom right) respectively.
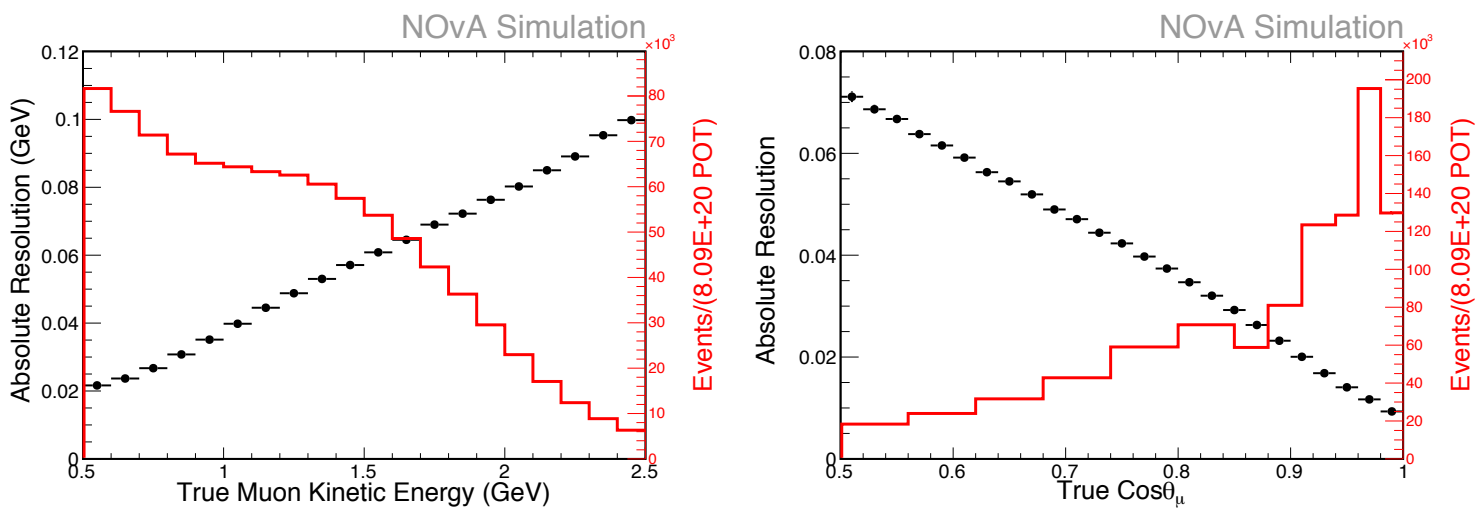

Figure 6.23: Left Figure: Black dot: Sigma and its error from Gaussian fit to the peak of Reco True K.E. with respect to True K.E. of muon. Red line: Reconstructed KE of muon. Right Figure: Black dot: Sigma and its error from Gaussian fit to the peak of Reco - True Cosine angle of muon with respect to True Cosine angle of the muon. Red line: Reconstructed cosine angle of the muon. 

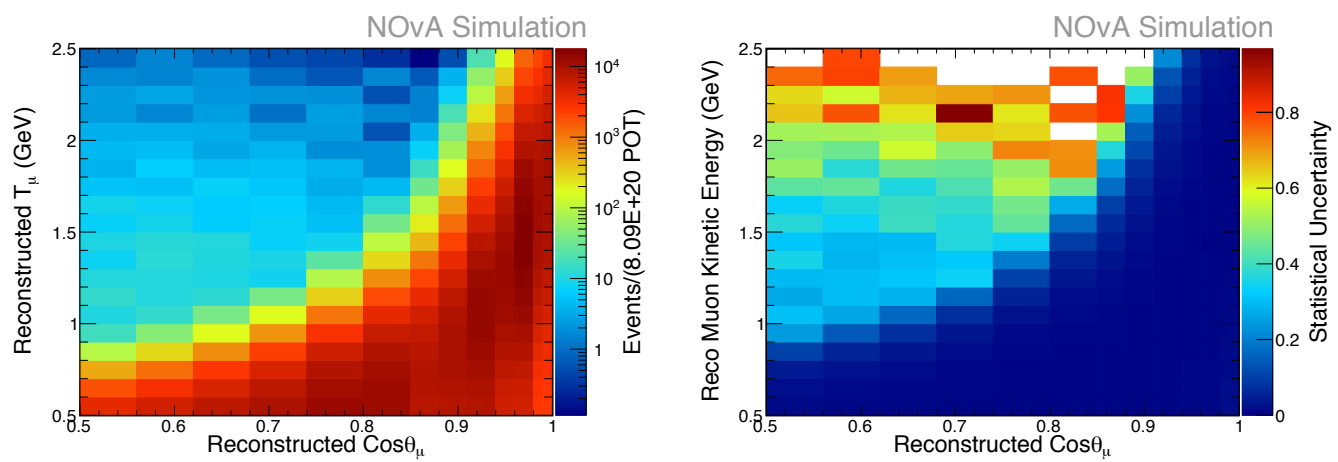

Figure 6.24: Reconstructed $T_{\mu}$ vs Reconstructed $\cos \theta_{\mu}$ with variable binning in $\cos \theta_{\mu}$ is 5 bins between $0.5-0.8,1$ bins between $0.8-0.85,3$ bins between $0.85-0.94,2$ bins between $0.94-0.98$, and 3 bins between $0.98-1.0$ and for $T_{\mu}$ is 20 bin for $0.5-2.5 \mathrm{GeV}$.
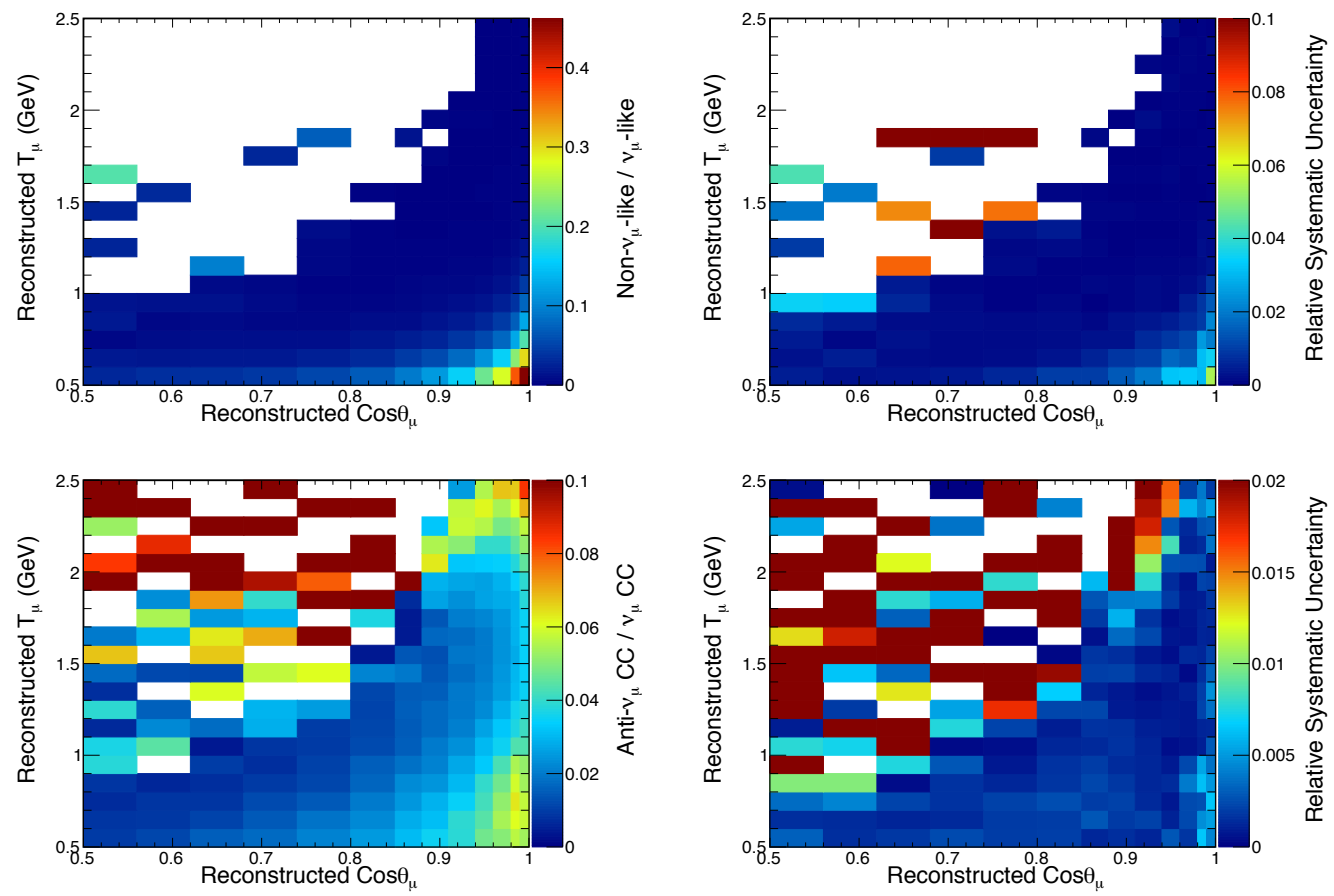

Figure 6.25: The non- $\nu_{\mu}$-like to $\nu_{\mu}$-like fraction (top left) and the systematic uncertainty on the non- $\nu_{\mu}$ like to $\nu_{\mu}$-like fraction in the kinematic phase space of muon (bottom left). The fraction wrong sign $\left(\nu_{\mu} \mathrm{CC}\right.$ to $\left.\overline{\nu_{\mu}} \mathrm{CC}\right)$ contamination and it's systematic uncertainty on the wrong sign fraction is shown in top right and bottom right respectively. 

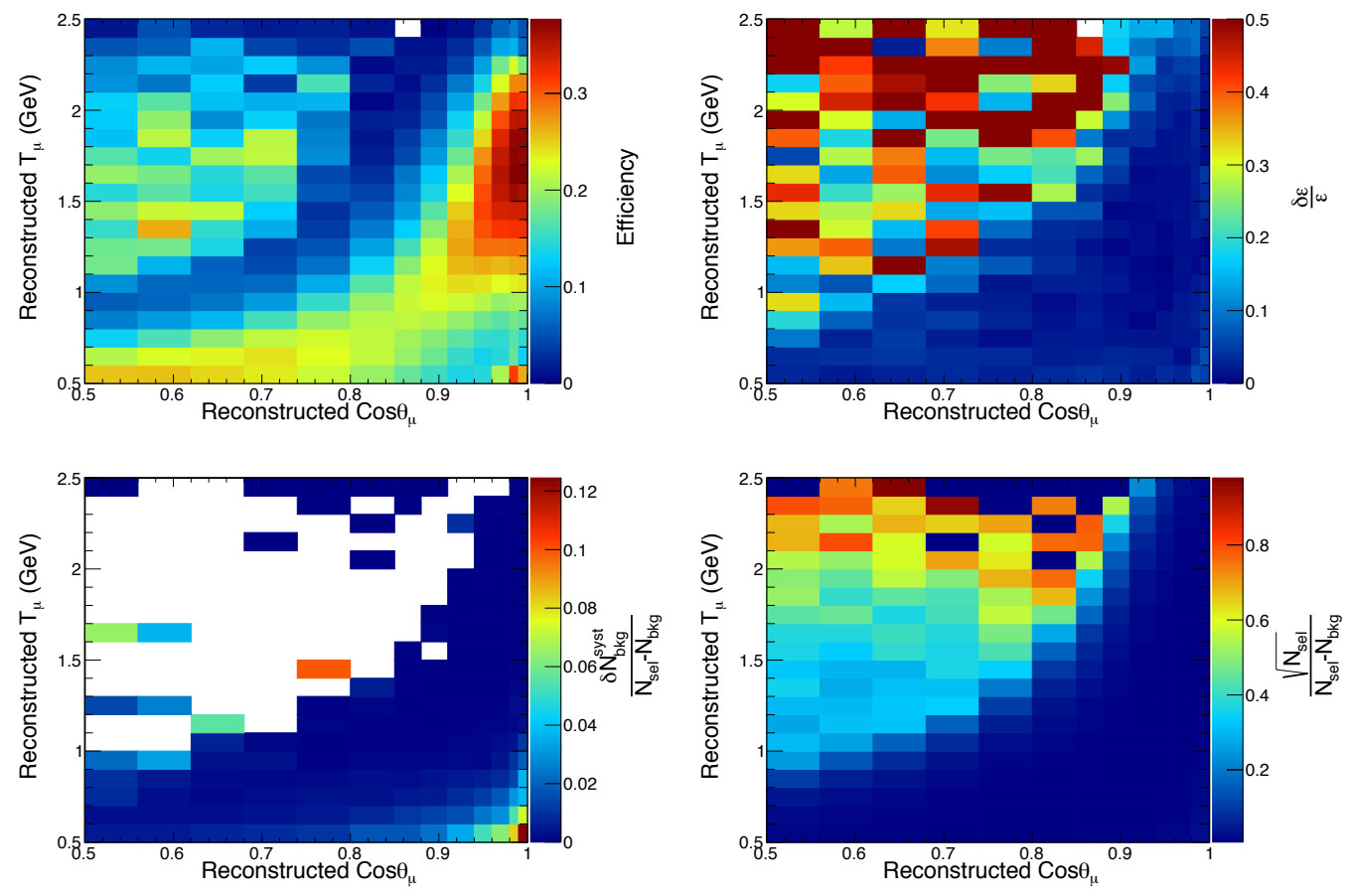

Figure 6.26: The top left figure shows the efficiency as a function of reconstructed muon kinematic phase-space. The relative uncertainty on efficiency is shown in the top right figure. The systematic uncertainty on background events to background subtracted signal event (bottom left) and the statistical uncertainty on signal events to background subtracted signal event in bottom right figure. Each pieces are goes into the total uncertainty on cross-section. 


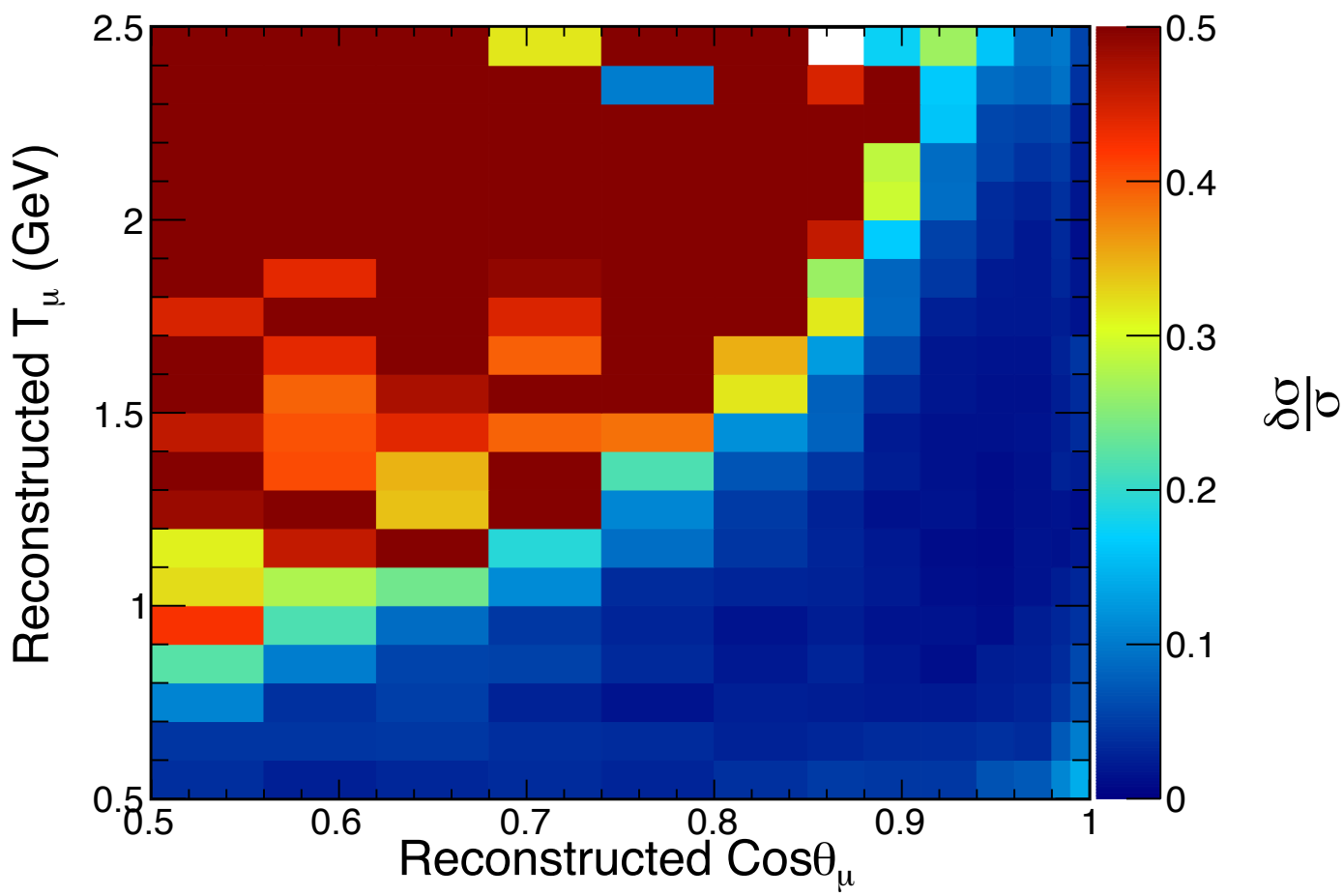

Figure 6.27: The fractional uncertainty on total cross-section as a function of kinematic phase space of muon.

the MuonID, as a function of the same true variables; the denominator in this case is true signal interactions with all but the PID cut applied. This shows that most of the dependence of efficiency on these true variables come from reconstruction and containment. Once an event passes those criteria, the PID's performance is fairly unbiased across the entire range represented here. Events with very high inelasticity are an exception. In these events, the muon is usually too short, or completely engulfed in hadronic activity and difficult to identify.

There are concerns that the efficiency determined from simulation will not accurately reflect reality. The observed distributions in data and simulation could be different for three reasons:

1. The underlying true distributions are different

2. The acceptance or efficiency are not modeled correctly in simulation

3. The relationship between the reconstructed and the true quantities is different between data and simulation

To investigate the first two concerns, a study was performed to measure the efficiency of selecting fiducial events in data and simulation in reconstructed space. This study is detailed in [105] and indicated that the efficiency of selection of events in the same reconstructed $\cos \theta_{\mu}$ bin is different in data and MC, and the difference is outside the $1 \sigma$ cross-section systematic uncertainty band. Followup studies (see $[106,107]$ ) explored the efficiency as a function of $\cos \theta_{\mu}$ in different hadronic and muon energy regimes and showed that the discrepancy is most severe for low-energy reconstructed muon candidates. 

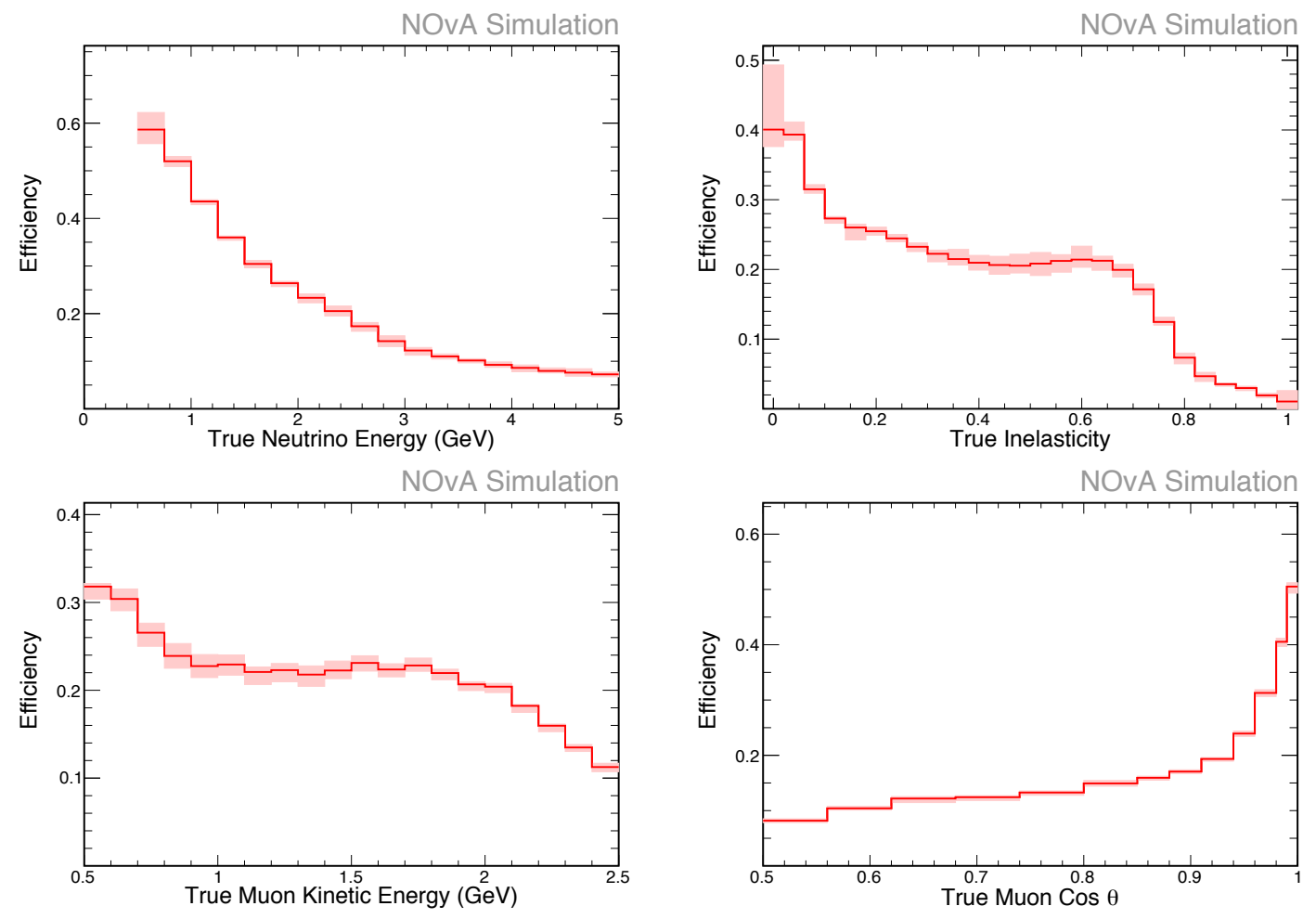

Figure 6.28: Total efficiency as a function of true quantities- neutrino energy (top left), inelasticity (top right), muon kinetic energy (bottom left) and muon $\cos \theta$ wrt beam direction (bottom right). The band is systematic uncertainty due to calibration, light-level, Cherenkov efficiency, flux uncertainty due to hadro-production and GENIE cross-section uncertainty. 

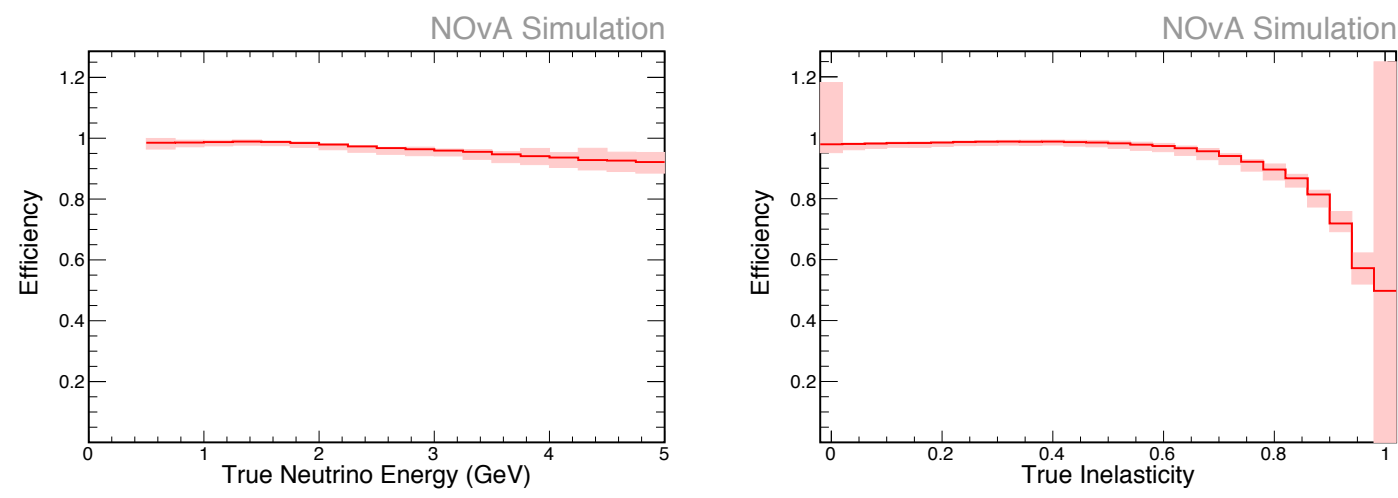

NOvA Simulation
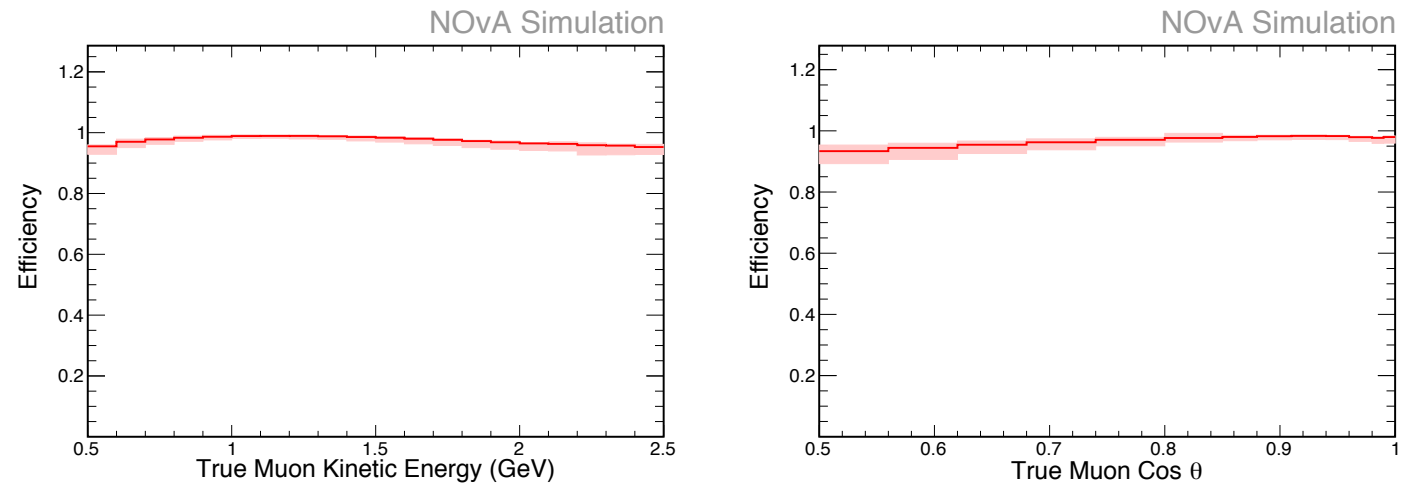

Figure 6.29: Efficiency of PID wrt to all other cuts, as a function of true quantities- neutrino energy (top left), inelasticity (top right), muon kinetic energy (bottom left) and muon $\cos \theta$ wrt beam direction (bottom right). The band is systematic uncertainty due to calibration, light-level, Cherenkov efficiency, flux uncertainty due to hadro-production and GENIE cross-section uncertainty. 


\subsubsection{Multidimensional Approach to Efficiency Correction}

In the traditional approach, the efficiency correction is computed and applied in the space in which the measurement is made. For example, for a cross-section measurement as a function of neutrino energy, the efficiency correction is expressed as a function of a single variable, the neutrino energy, and all information of its dependence on other quantities is lost. This approach can be problematic when the efficiency depends strongly on other quantities that are different between data and simulation.

In the measurement of the cross-section in the double-differential muon kinematic space, the efficiency correction is applied in the 2D space and naturally accounts for the observed differences in muon kinematics in the data and simulation, provided that those discrepancies arise due to different underlying true distributions. To capture this dependence in the cross-section measurement as a function of neutrino energy, it is proposed that for both, the energy-dependent and the doubledifferential measurements, the efficiency correction is done in the 3D space of neutrino energy, muon kinetic energy and muon $\cos \theta$ with respect to the mean beam direction. The selection efficiency depends on hadronic energy too, but this dependence is appropriately captured in the neutrino and muon energy space, since $E_{h a d}=E_{\nu}-E_{\mu}$.

In this scheme, the reconstructed spectrum from data is generated, unfolded and efficiency corrected in 3D. Once the corrected 3D "true" data distribution is available, the distribution is projected to an appropriate axis/axes of the measurement.

\subsection{Unfolding}

Unfolding procedures are commonly used in high energy physics to correct for the known effects of measurement resolutions and systematic biases in the reconstruction to extract the true distribution. In the case of this analysis, unfolding is performed on distributions of reconstructed observables after background subtraction from the selected events. The input to unfolding algorithms is a $2 \mathrm{D}$ correlation between the true and reconstructed versions of a given variable for simulated events (see Figs. 6.30 and 6.31). This matrix needs to be inverted to allow a mapping from the reconstructed variable to the true value, and a simple inversion can introduce unphysical results due to unavoidable low statistics in certain bins and other rare processes that cause migration across reconstructed bins. The goal of the unfolding algorithm is to dampen the terms that produce these unphysical results, and unfolding algorithms such as "Iterative" [108], singular value decomposition (SVD) [109], Tikhonov regularization [110], and maximum entropy deconvolution (MAXED) [111]) each take a different approach. However, in general each algorithm has a parameter that is adjusted to get the best possible match between the unfolded data set and the underlying true distribution. In this analysis, we have investigated two unfolding algorithms: Iterative and SVD.

An optimization test, based on the average correlation is to see which parameter will be used which can be the best match between unfolded and true distribution for this analysis. For this study, fake data (normalized to an exposure of $8.09 \times 10^{20}$ POT and statistically independent half of the total MC dataset) is used to test the unfolding performance of the two algorithms under consideration.

$$
\text { Average Correlation }=\sqrt{1-\left(\left(V_{x x}\right)_{j j}\left(V_{x x}\right)_{j j}^{-1}\right)^{-1}}
$$




\section{NOvA Simulation}

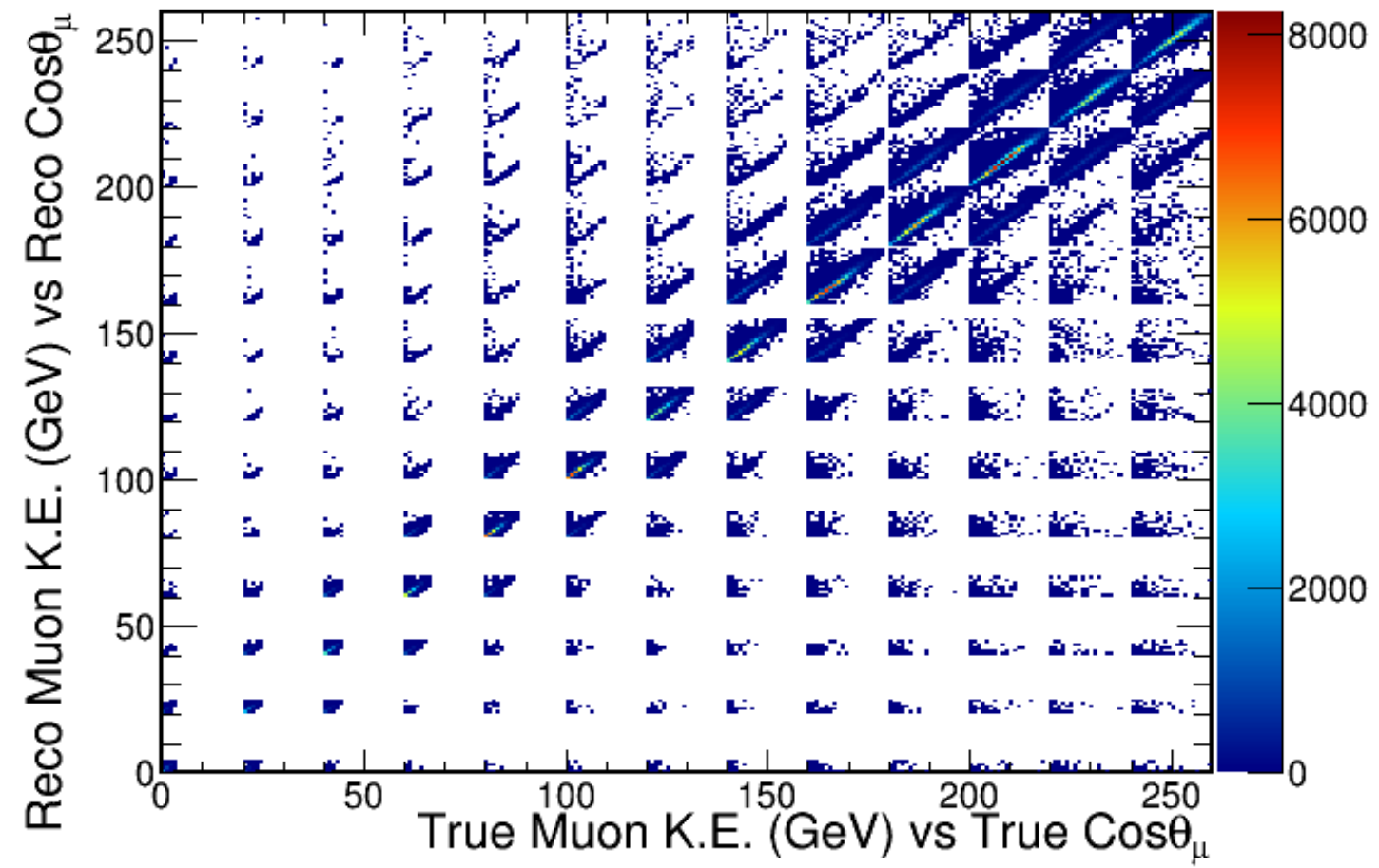

Figure 6.30: The migration matrix (true vs. reco) for the double-differential (right) measurement bins. 


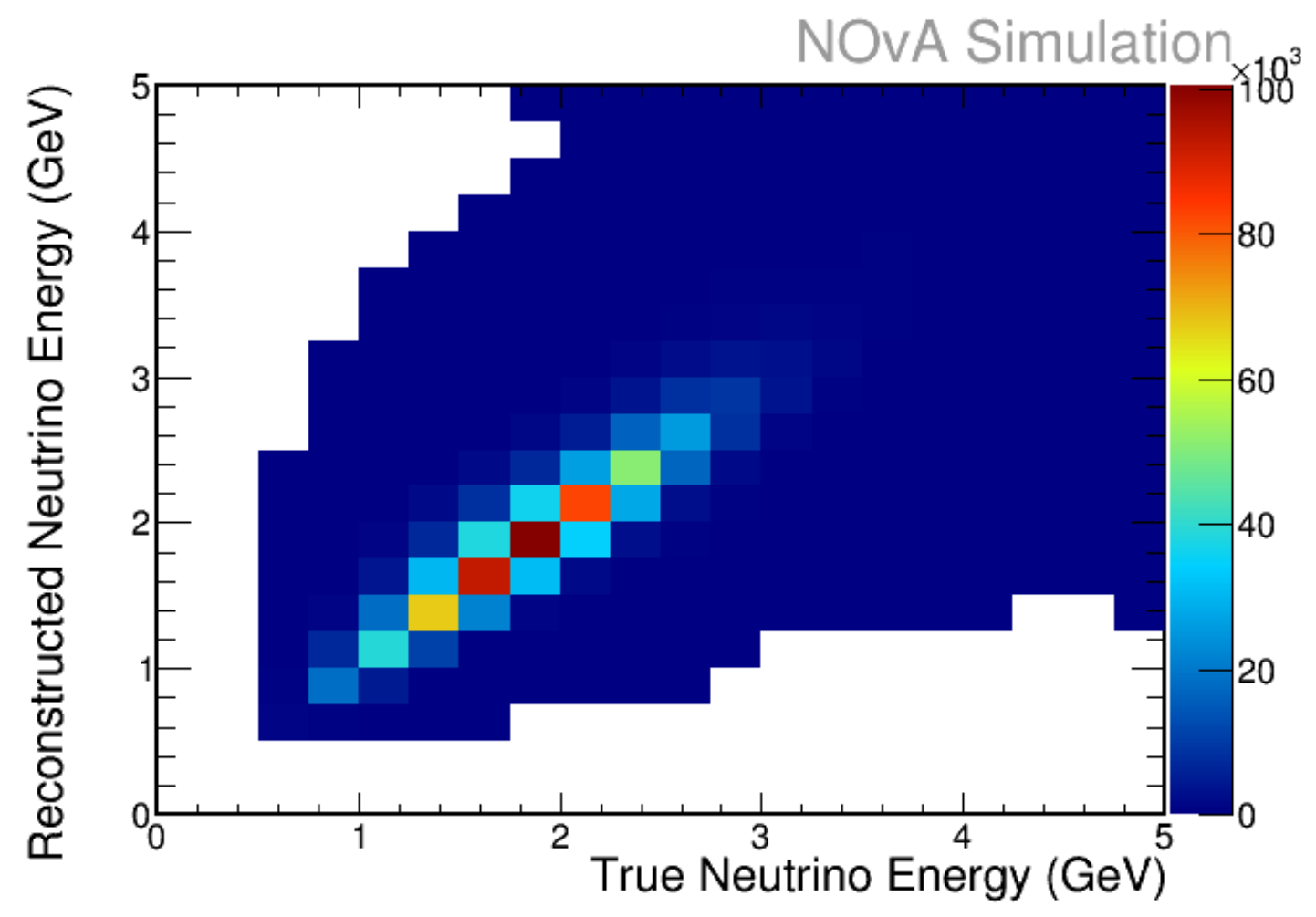

Figure 6.31: The migration matrix (true vs. reco) for the reconstructed neutrino energy. energydependent (left) and double-differential (right) measurement bins. 

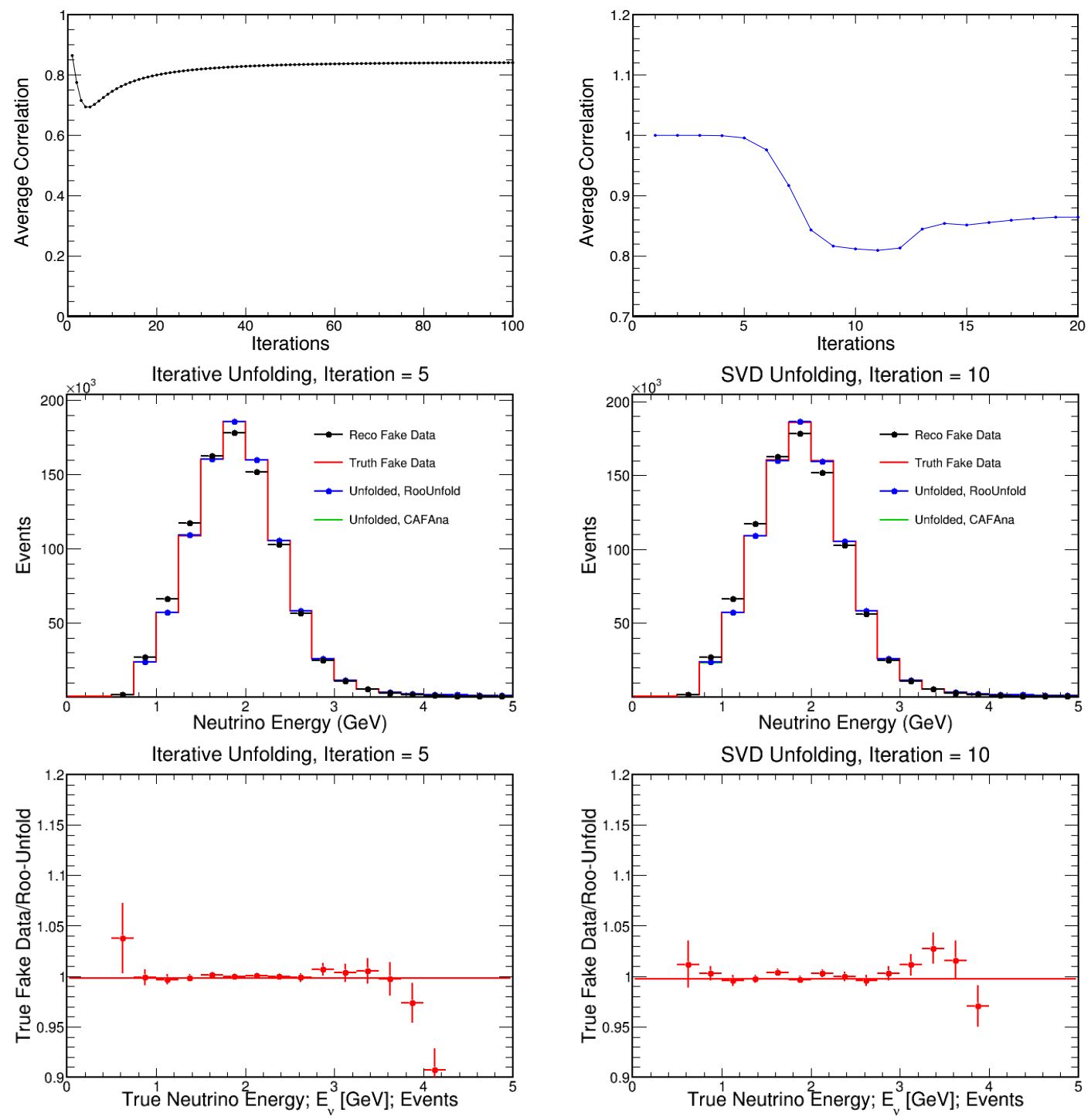

Figure 6.32: A comparison of the Iterative (left) and SVD (right) unfolding algorithms using a statistically independent, but otherwise unmodified, fake data set. Unfolding was done on signal only (perfect background subtraction). Cross-section and PPFX central value weights are applied. The average correlation as a function of iteration for each algorithm is shown in the top plots. The middle plots show the reconstructed, true and unfolded neutrino energy spectra. The unfolding was done using both CAFAna and RooUnfold tools, and they are in perfect agreement. The ratio between true and unfolded distributions are shown in the bottom plots. 

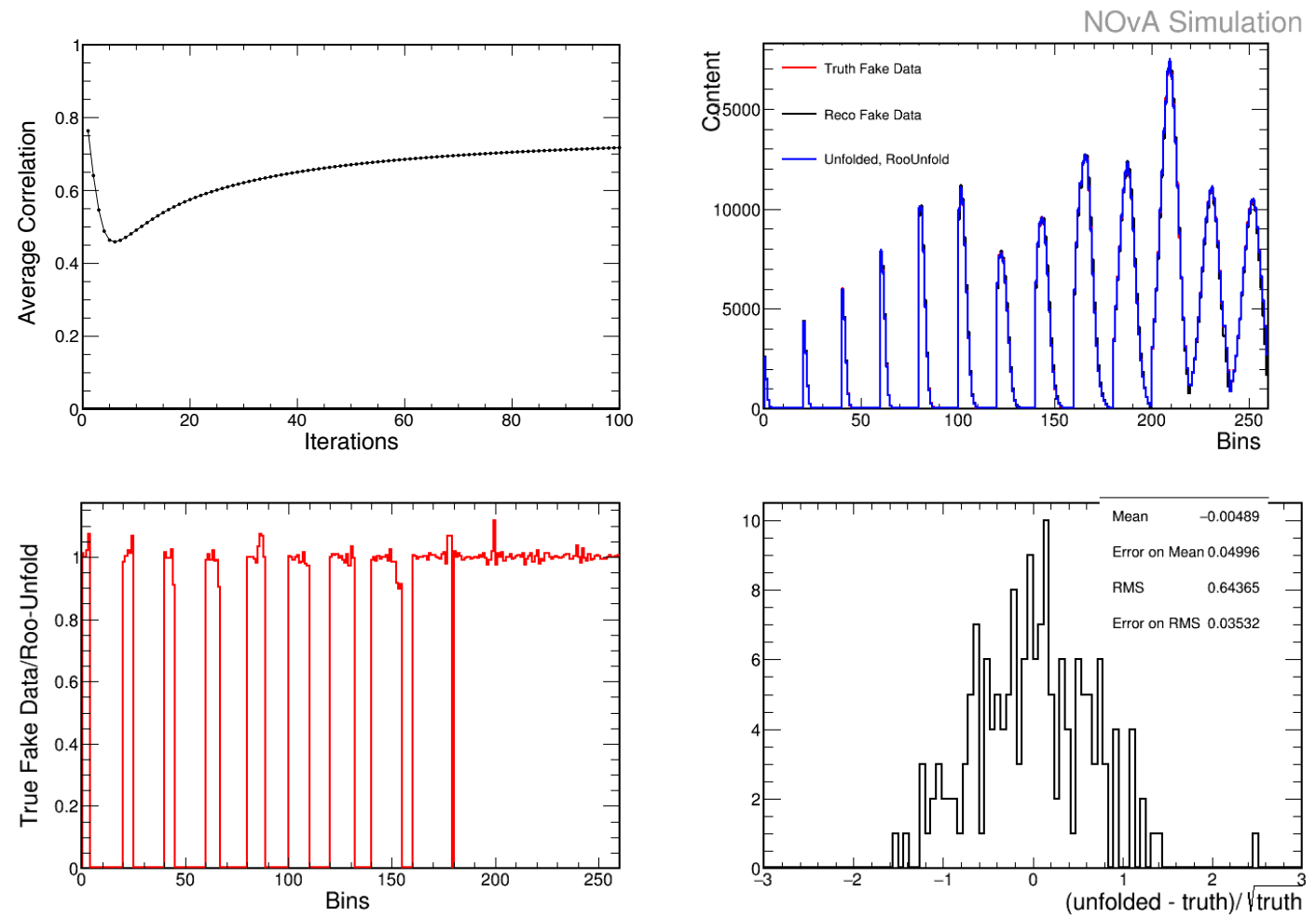

Figure 6.33: Top left: The average correlation as a function of iteration for the Iterative unfolding algorithm for the double-differential measurement bins. Top right: The reconstructed, true and unfolded neutrino energy spectra. Bottom left: The ratio between true and unfolded distributions. Bottom right: The distribution of the weighted difference between the unfolded and true values in each measurement bin.

where $\left(V_{x x}\right)_{j j}$ is the diagonal covariance matrix (covariance between the truth bins $(\mathrm{j})$ ). The number of iteration that gives the minimum value of the average correlation is chosen in order to reduce the effects of smearing. The details about this metric are found in reference [108].

We compare the performance of the unfolding algorithms for this analysis using simulated fake data sets. Figure 6.32 shows a comparison of unfolding fake data sets that are simply statistically independent from the simulation. The Iterative algorithm finds a minimum average correlation of approximately 0.68 after 5 iterations; the SVD algorithm minimum is 0.8 after 10 iterations. The performance of the Iterative unfolding algorithm on the same statistically independent fake data set on the double-differential cross-section bins is shown in Fig. 6.33. In this case, the minimum average correlation is 0.45 after 6 iterations.

In another study, we generate a fake dataset where a $Q^{2}$-dependent weight is applied to the signal events. The weight as a function of $Q^{2}$ is shown in the left plot of Fig.6.34, and the impact of the weight on the energy spectrum is shown in the right plot of the same Figure. Figure 6.35 shows the performance of the Iterative and SVD unfolding algorithms under this scenario, and Fig. 6.36 shows the performance of the Iterative algorithm on the double-differential cross-section measurement bins. The apparent bias between the unfolded and truth in the double-differential bins is under investigation.

In the case of unfolding the energy spectrum, the Iterative algorithm results in the smaller average 

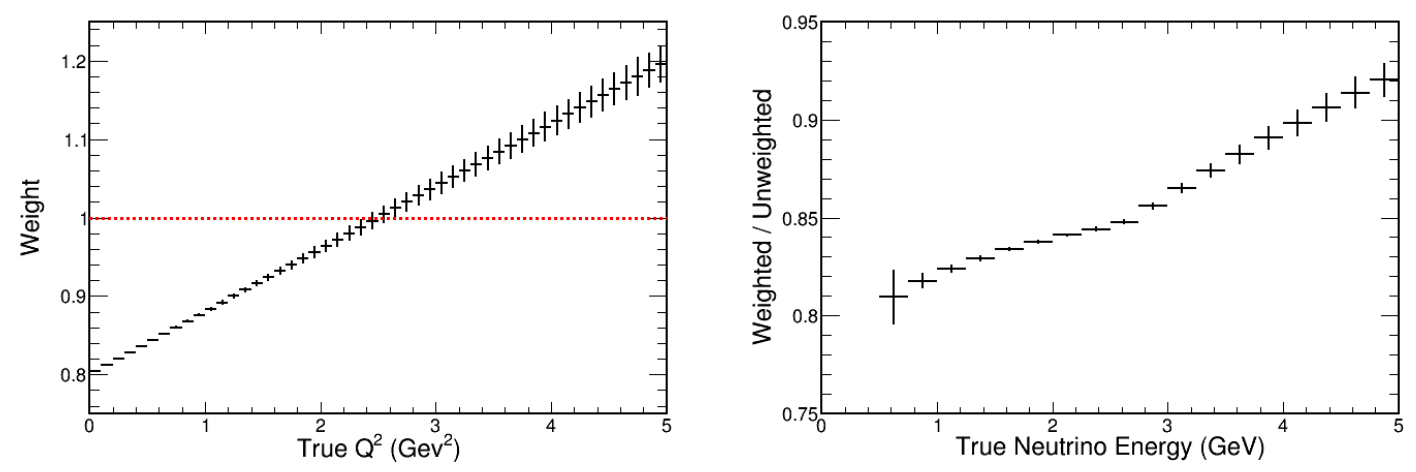

Figure 6.34: The left figure shows the weight applied to the fake data events, as a function of the true $Q^{2}$. The right figure shows the ratio of the weighted and the unweighted fake data spectrum as a function of the neutrino energy space.

correlation between bins. There also appears to be a better agreement between the unfolded and true energy spectra in both the unweighted and weighted fake data sets. On this basis, We choose to use the iterative unfolding algorithm for the analysis.

\subsection{Flux Calculation}

The flux of neutrinos varies across the surface of the detector and must be integrated properly over the fiducial volume. In CAFAna, the flux is computed by inverting equation 5.1 using simulation:

$$
\phi(E)=\frac{N(E)_{s i g}}{N_{\text {target }} \frac{d \sigma(E)}{d E}}
$$

where $\sigma$ is the true cross-section that GENIE records for every interaction it generates, and $N_{\text {sig }}$ is the total number of true signal interaction that occurred in the fiducial volume. The derivation of this approach is discussed briefly in [112]. For this method to work, a particular GENIE process must be selected to base the flux on. Therefore, the limitation of computing the flux by this method is that it is not possible to assess the flux at neutrino energies below the energy threshold for the GENIE process used in the method. For the flux used in this analysis, the process used is quasi-elastic CC interactions of $\nu_{\mu}$ on $C_{12}$ nuclei in the fiducial volume. Every interaction reweighted by the $\mathrm{ppfx}$ weights to get the flux constraint by the external hadron production data. The same procedure is made in each universe to get the corresponding flux and calculate the beam uncertainties.

Figure 6.37 shows the flux integrated in a fiducial volume calculated with this procedure. The upper plot is the flux spectrum corrected by PPFX with the error band corresponding to the hadron production and focusing systematics. The lower plot is the ratio between the corrected flux over the prediction without any correction. The effect of the correction to the MC model (G4NuMI) is to reduce the beam by $10-12 \%$ and it is almost flat in the neutrino energy. 

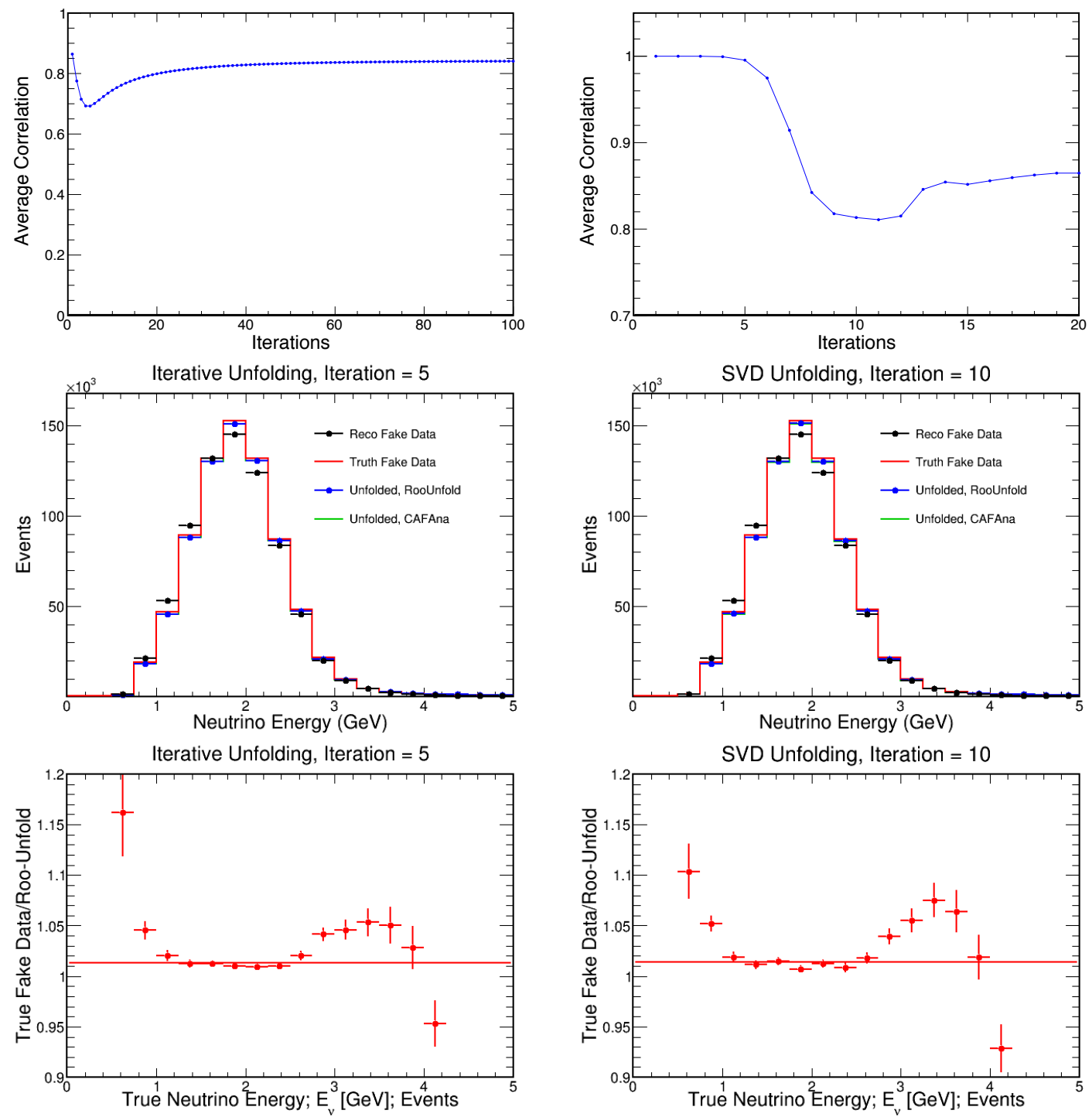

Figure 6.35: A comparison of the Iterative (left) and SVD (right) unfolding algorithms using a statistically independent, fake data set with the weights shown in Fig.6.34 applied. Unfolding was done on signal only (perfect background subtraction). Cross-section and PPFX central value weights are applied. The average correlation as a function of iteration for each algorithm is shown in the top plots. The middle plots show the reconstructed, true and unfolded neutrino energy spectra. The unfolding was done using both CAFAna and RooUnfold tools, and they are in perfect agreement. The ratio between true and unfolded distributions are shown in the bottom plots. 

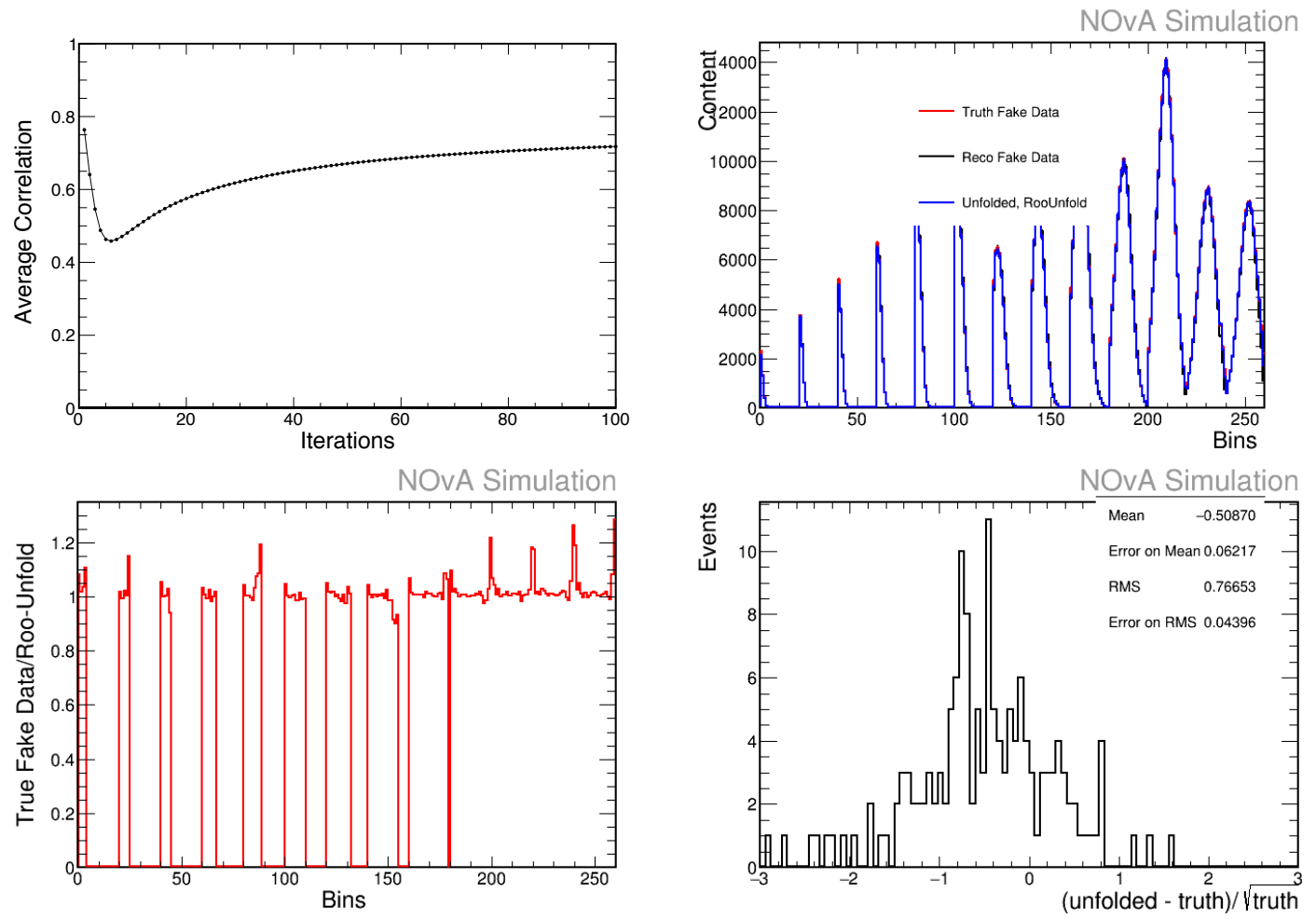

Figure 6.36: In this Figure $Q^{2}$ weight is applied to fake data in addition to cross-section and PPFX central value weight. Average correlation as a function of iteration, optimized iteration number is 6 (top left). The kinematic variable (2D) is collapse into 1D distribution for reconstruction fake data (already background subtracted), true fake data and unfolded distribution (top right). The ratio between true fake data to unfolded distribution (bottom left). The weighted difference between unfolded and true distribution with mean and rms are reported.(bottom right) 


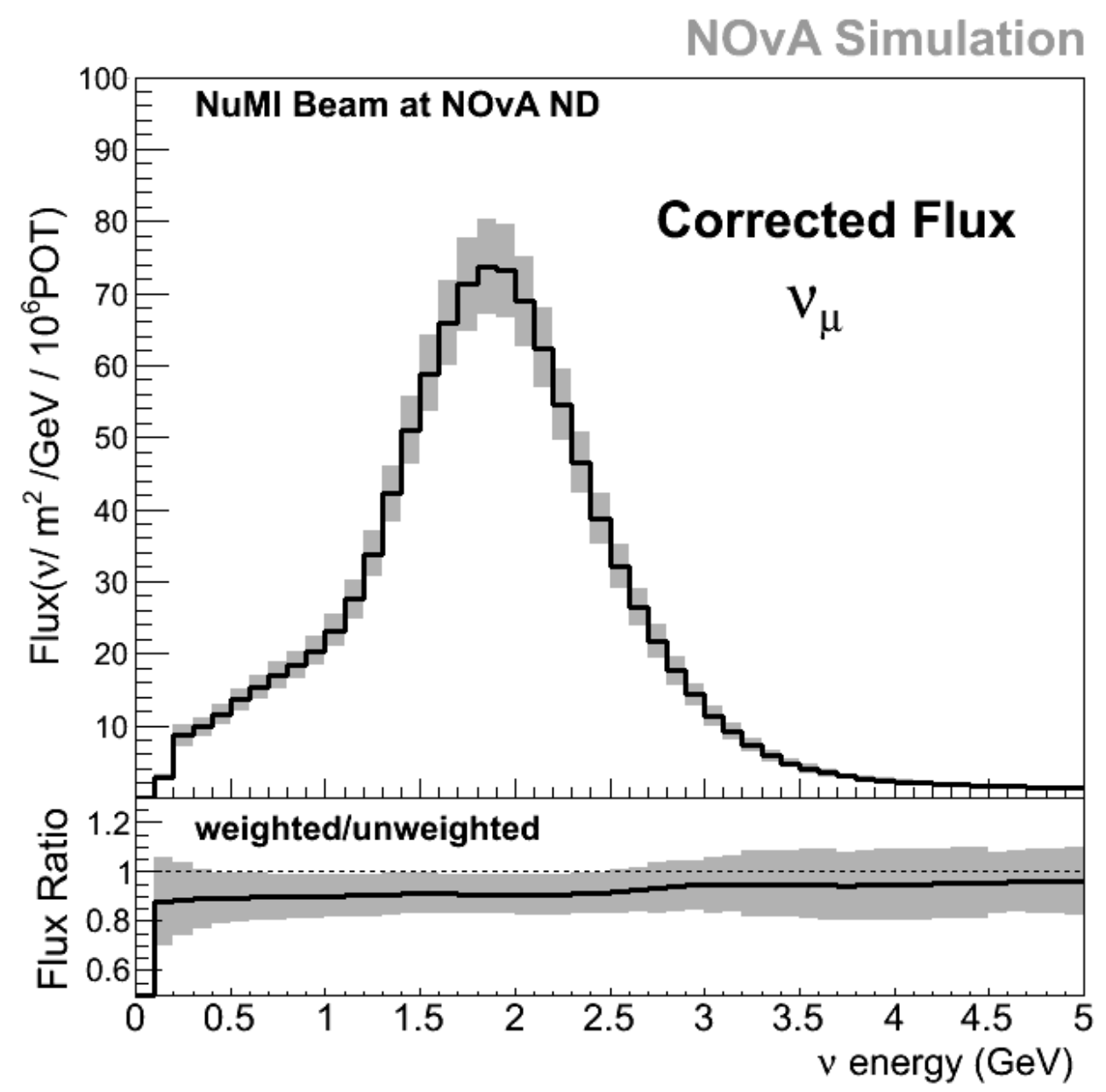

Figure 6.37: Muon neutrino beam integrated into a volume slightly wider than the fiducial one used in this analysis ( $\mathrm{X}$ in $[146,147.45] \mathrm{cm}, \mathrm{Y}$ in $[142,149.45] \mathrm{cm}$ and $\mathrm{Z}$ in $[25,1150] \mathrm{cm}$ ). The uncertainties correspond to the hadron production and beam focusing systematics. The upper plot is the flux spectrum corrected by PPFX. The lower plot is the size of the correction respect to the default beam model. 


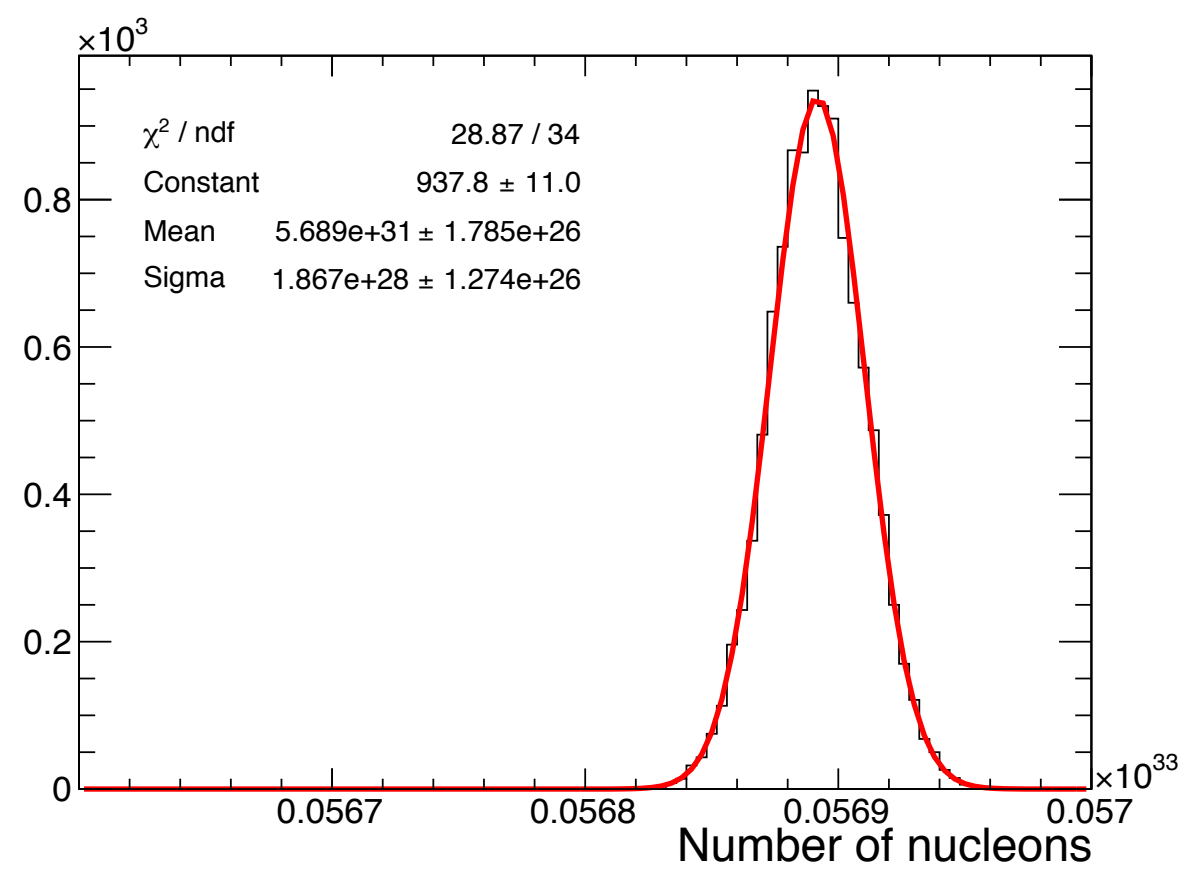

Figure 6.38: Distribution of the number of nucleon targets obtained by 10,000 repetitions of the random poll algorithm, with a Gaussian fit.

\subsection{Target Counting}

The number of nucleon targets in the fiducial volume is estimated using a 3D random-poll algorithm. The accuracy of this method depends on the number of test points, $n$. A 3D point is randomly placed in the geometry, inside the fiducial volume. A cubical volume of size (total detector volume divided by $n$ ) is assumed to be composed of the same material as the material at the location of the $3 \mathrm{D}$ point, and its mass is taken to be the cubical volume times the density of that material. This process is repeated $n$ times, and the masses of all the $n$ cubical volumes added to get an estimate of the detector mass in the fiducial volume.

The GDML used here is neardet-3x3-8block-xtru-vacuum-stagger.gdml, from release S17-06-30, which contains updates to cell geometry and material compositions listed in [113] and represents our best knowledge of detector geometry. The results from one run of the random-poll algorithm, with a million test points, are listed in Table: 6.4 for detector materials and in Table: 6.5 for elemental composition. The total mass is computed to be $94561.5 \pm 580.5 \mathrm{~kg}$. The total number of nucleons is obtained by multiplying the total mass (in grams) by the Avogadro's number.

Since the random poll is a stochastic algorithm, each run produces a slightly different outcome. To get a more robust estimate of target count, the algorithm was run 10000 times, and a Gaussian fitted to the resulting distribution of target counts (Figure: 6.38). The mean target count was found to be $5.689 \times 10^{31} \pm 1.785 \times 10^{26}$ (error on mean from the fit) nucleons. 


\begin{tabular}{llllll}
\hline Material & Density $\left[\mathrm{g} / \mathrm{cm}^{3}\right]$ & Volume $\left[\mathrm{cm}^{3}\right]$ & Mass $[\mathrm{kg}]$ & Fraction of Total & Error in mass $[\mathrm{kg}]$ \\
\hline Air & 0.001205 & $2.9558 \mathrm{e}+06$ & 3.56174 & $3.76639 \mathrm{e}-05$ & 0 \\
Glue & 0.98 & 685738 & 672.024 & 0.00710636 & 33.6012 \\
PVC & 1.4947 & $2.31552 \mathrm{e}+07$ & 34610.1 & 0.365987 & 553.761 \\
Scintillator & 0.8576 & $6.91241 \mathrm{e}+07$ & 59280.9 & 0.626869 & 355.685 \\
\hline
\end{tabular}

Table 6.4: Mass contributions from different materials in the fiducial volume

\begin{tabular}{llll}
\hline $\mathrm{Z}$ & Mass $[\mathrm{kg}]$ & Fraction of Total & Uncertainty $[\mathrm{kg}]$ \\
\hline 1 & 10157.4 & 0.107415 & 78.8064 \\
6 & 63003.6 & 0.666271 & 515.42 \\
7 & 24.3881 & 0.000257908 & 0.171106 \\
8 & 2829.31 & 0.0299203 & 50.663 \\
11 & 2.46078 & $2.6023 \mathrm{e}-05$ & 0.0393724 \\
16 & 90.9212 & 0.000961503 & 1.52707 \\
17 & 15263 & 0.161408 & 245.293 \\
20 & 24.9331 & 0.000263671 & 0.39893 \\
22 & 3057.59 & 0.0323344 & 48.9214 \\
50 & 112.974 & 0.00119472 & 1.80759 \\
\hline
\end{tabular}

Table 6.5: Mass contributions from different elements in the fiducial volume

\subsection{Additional Systematics}

\subsubsection{Target Count + POT}

The error on the total target count is taken from the geometry technical note [113], which accounts for the known uncertainties in the measurement of masses and volumes during the construction of the detector. It estimates an uncertainty of $0.27 \%$ on scintillator mass, $0.59 \%$ on PVC mass and $5 \%$ on the mass of the Glue in the ND. Added in quadrature, these result in a $0.28 \%$ uncertainty on the total mass of the detector, which is applied as a simple normalization uncertainty. Given the smallness of this uncertainty, no attempt is made to account for the uncertainty arising due to this on the elemental compositions, which would also modify the cross-section.

The uncertainty on the POT accrual in the NuMI beamline is $0.5 \%$ and is also included as a normalization uncertainty.

\subsubsection{Intensity Effects}

The efficiency of selecting $\nu_{\mu}$ CC interactions is dependent on the beam intensity due to the limited ability of the slicer to handle pileup. The selection efficiency as a function of beam intensity has been studied in [114]. The study shows that there is a small difference $(\sim 2 \%)$ in the response to pile up in the data and MC, which we will include as a normalization uncertainty (see Fig. 6.39).

\subsubsection{Muon Catcher Steel}

Uncertainties in the density and thickness of the muon catcher steel are summarized in [115]. Although the typical uncertainty of the product of density and thickness is $0.5 \%$, we plan to shift the amount of energy of the muon attributed to the segment of the reconstructed track in the muon 

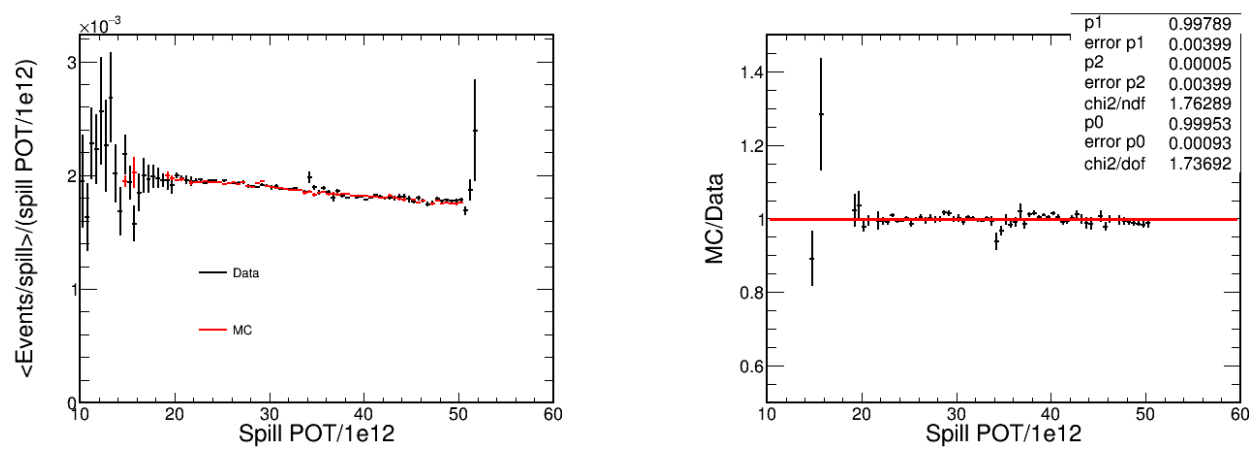

Figure 6.39: The ratio between number of selected slices from the recTree and number of spills from spillTree provides mean of Events/spill distribution as a function of Spill POT in data and MC (left) and data/MC ratio as well as linear and constant Fit results are reported (right).

catcher by this amount to estimate the systematic impact on the reconstructed energy. The impact of this uncertainty on the signal selection is negligible.

\subsubsection{Alignment}

Based on studies done for the 2015 and 2016 oscillation analyses[116], the systematic uncertainty due to misalignments of the PVC cells in the ND is $<1 \%$ on both the selection efficiency and reconstructed energy. This uncertainty is not included in the measurement.

\subsubsection{Particle Propagation Model}

The alternative GEANT physics lists had almost 1-2\% effect on most reconstructed distributions. The effect on the reconstructed neutrino energy, the reconstructed kinetic energy of muon and the cosine of the angle with respect to NuMI beamline has shown in Fig.6.40 and 6.41 respectively. All Distributions are normalized by 4E20 POT. Black line, Red bullet and blue bullet shows nominal QGSP_BERT_HP, QGSC_BERT, and FTFP_BERT respectively. The bottom plot shows the ratio between nominal to alternative physics list. Red and Blue are nominal to QGSC_BERT and FTFP_BERT respectively. We have compared different physics list and we take a 1-2\% normalization uncertainty from the distributions. Detailed studies are in [117]. 


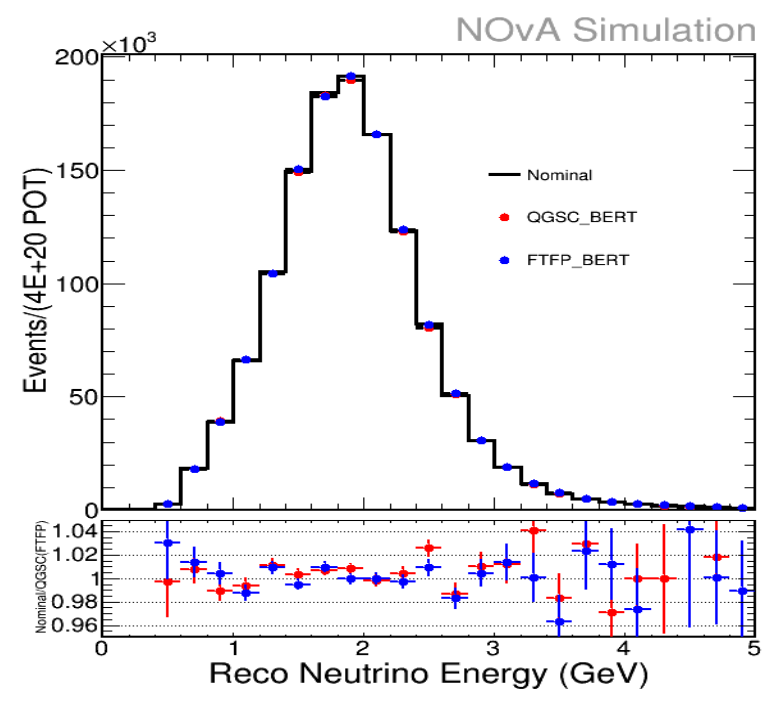

Figure 6.40: Reconstructed Neutrino Energy Distribution with Different Physics list
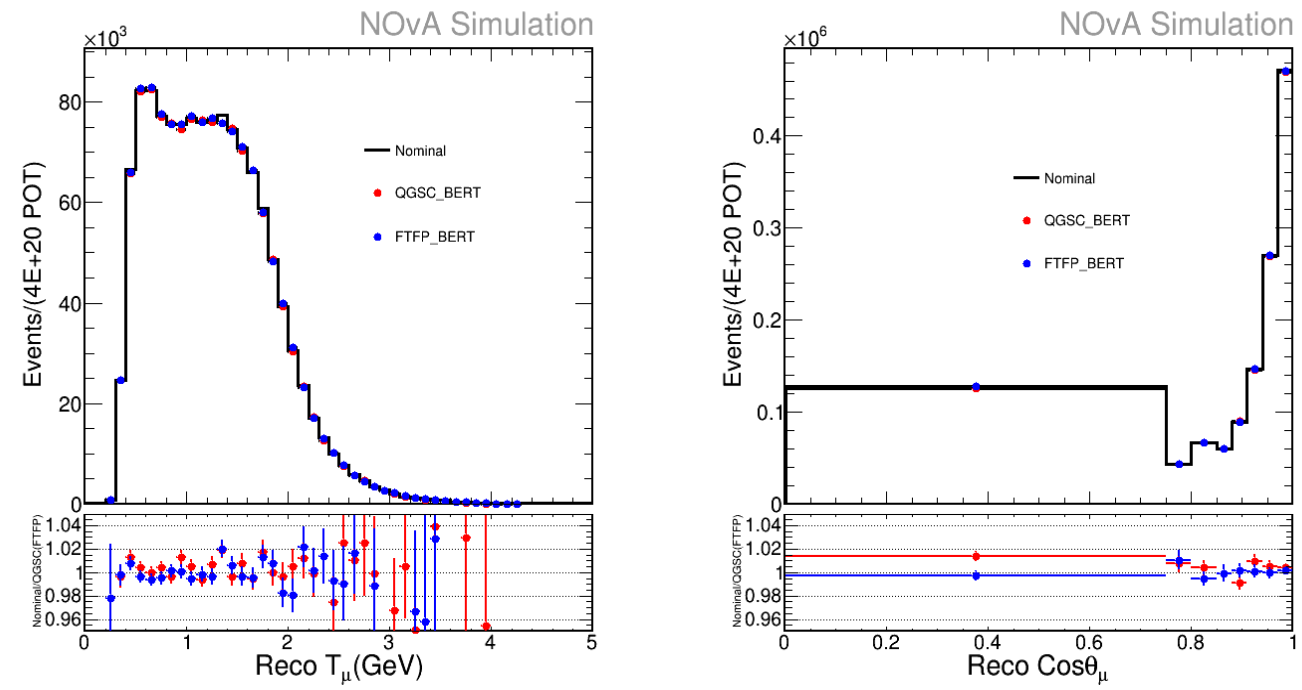

Figure 6.41: Reconstructed Muon Kinetic Energy Distribution with Different Physics list (Left), Reconstructed Cosine Angle of Muon Distribution with Different Physics list (Right). 


\section{Chapter 7}

\section{Results and Conclusion}

After unfolding and efficiency correction, the cross-section is easily computed by diving integrated flux and number of the targets. The flux-averaged double-differential cross-section measurement as a function of muon final state kinematics is shown in Fig. 7.1 and Fig. 7.2. Furthermore, the double differential cross-section is measured in each bin of the cosine angle of the muon, averaging the kinetic energy of muon. The overall agreement between data and GENIE (NOvA tuned) prediction can be seen in the figures. The GENIE (NOvA tuned) is underpredicted in the region of higher angle muons and in some of the bins of muon angle at higher kinetic energy GENIE (NOvA tuned) is overpredicted. Averaging the unfolded and efficiency corrected kinetic energy of muon, with respect to unfolded cosine angle of muon, is shown in Fig. 7.3. From this figure, it is clear that the data and GENIE (NOvA tuned) agreement is within $2 \%$ for the lower angle of muons and for higher angle of muons it is within $5 \%$ for most of the bins.

In those figures (Fig. 7.1 and Fig. 7.2), we have also compared raw predictions from GENIE (default), NuWro, NEUT and GiBUU generators with the GENIE (NOvA tune) and our data. The NuWro prediction is shown in green curve, NEUT prediction is in orange, magenta and cyan curve represent predictions from GiBUU and GENIE (default) generator respectively. In this case there is no phase space cut applied in the generator, so we saw a smooth curve. However, there is a sharp cut in the GENIE (NOvA tuned) (red curve) and data (black point) because of phase space cut. There is a close agreement between data and NuWro prediction in low cosine angle of muon. Similarly, NEUT prediction agrees with data for most of the bins in the cosine angle of muon. The prediction from GiBUU and GENIE (default) are under predicted from data points. The data shown here are with statistical and systematic uncertainties, where statistical uncertainties are from the data distribution (not from the unfolded distribution and we will implement same in the near future).

The total cross-section in the kinematic constrained phase space is measured as a function of neutrino energy, which is shown in Fig. 7.4. In this figure, the shape falls down after $2.6 \mathrm{GeV}$ of neutrino energy, as it is kinematically constrained. The overall data and GENIE (NOvA tuned) in each bin of neutrino energy agree pretty well. The comparison of other generator will be reported elsewhere.

As described, the inclusive muon neutrino charged-current double-differential cross-section is presented for the various angles of muons and also a kinematic constrained total cross-section as a function of neutrino energy. To reiterate, the data and GENIE (NOvA tuned) agree pretty well. 


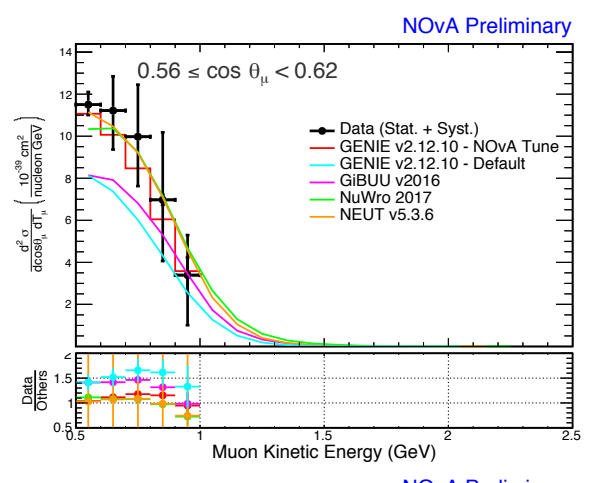

NOvA Preliminary

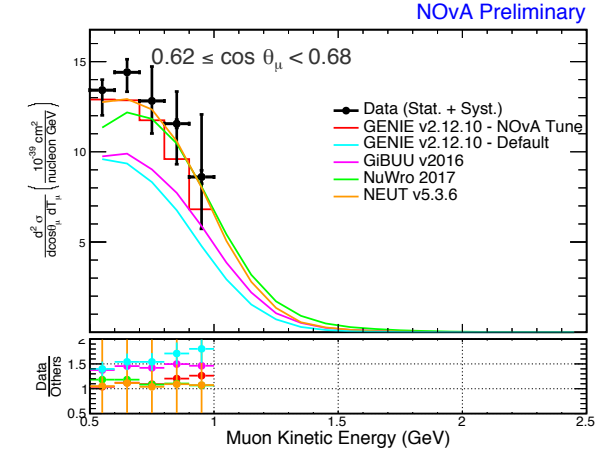

NOvA Preliminary

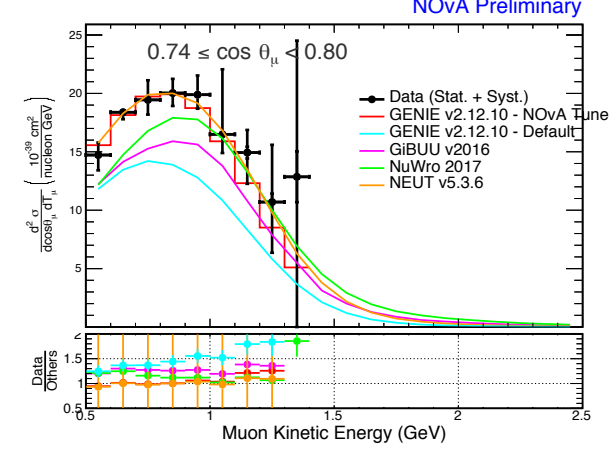

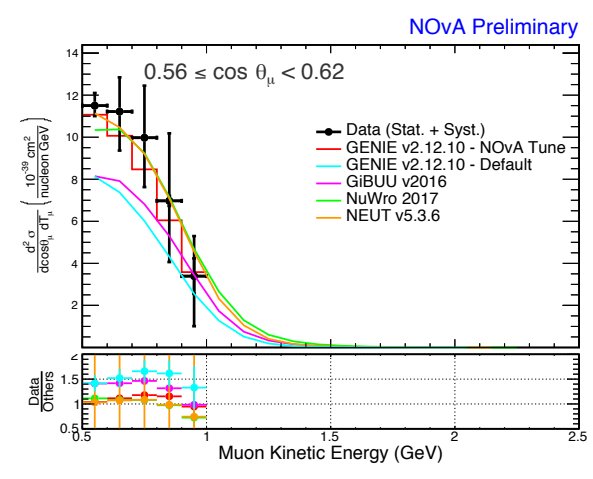

NOvA Preliminary
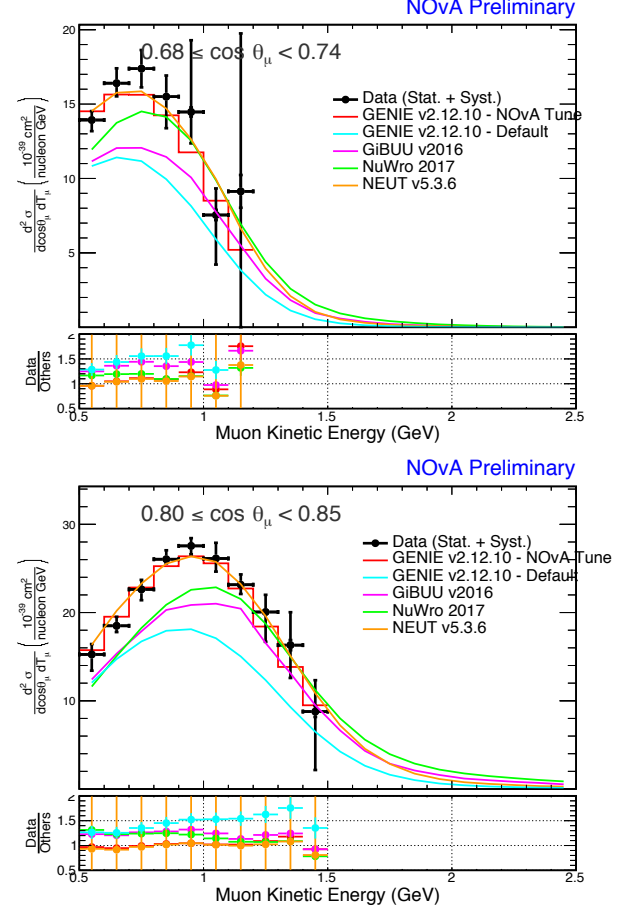

Figure 7.1: The double differential cross-section measurement for different angle of muons as a function of kinetic energy of muon. The data (statistical + systematic uncertainty) is shown in black and GENIE (NOvA tuned) prediction is shown in red and the bottom plot shows the ratio of data and other generator. In the bottom plot, the colors (for other generator) are divided from data points (black). The NEUT predictions show large statistical uncertainties, which are due to low statistics. The numerical value are quoted in the appendix. 

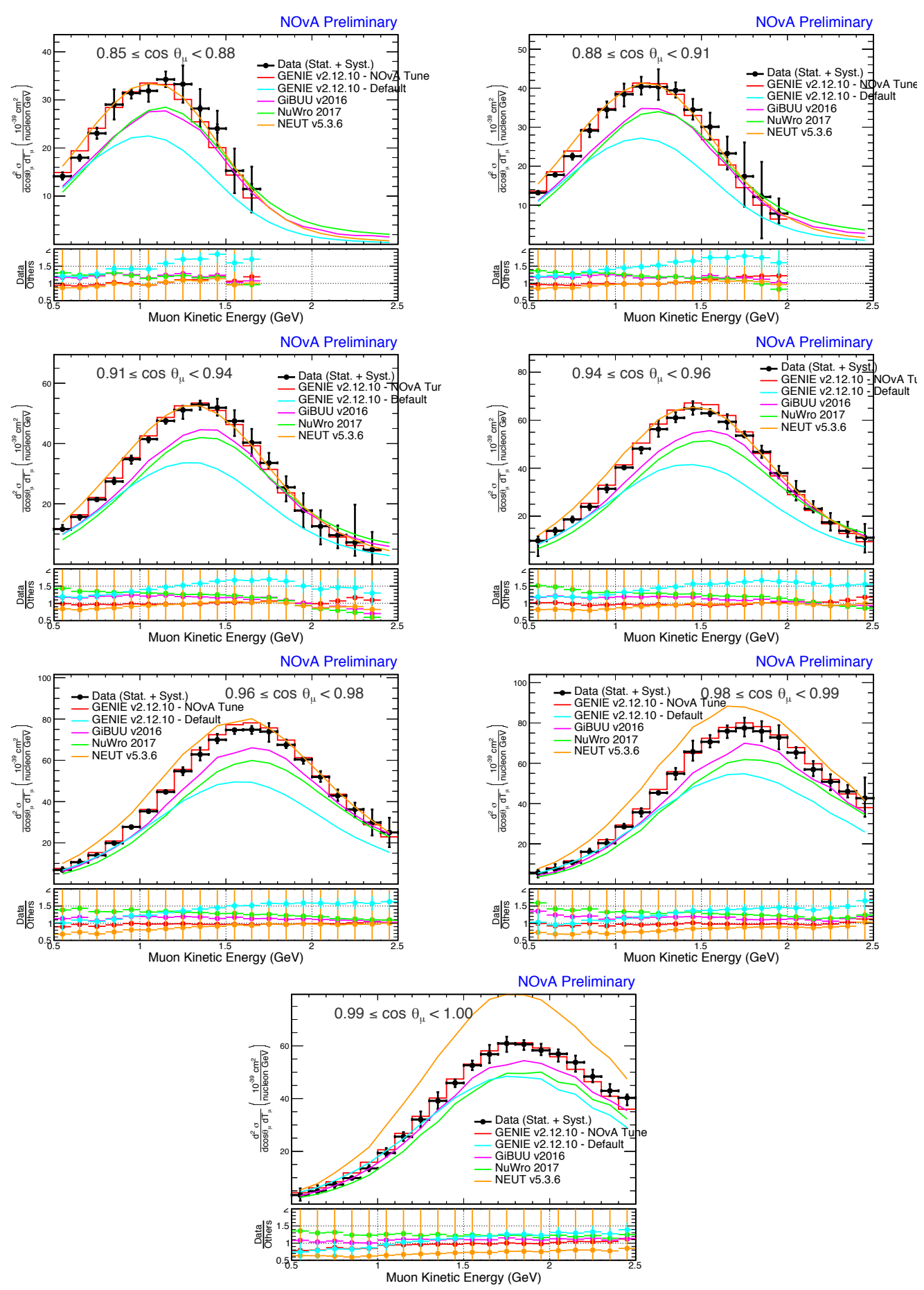

Figure 7.2: The double differential cross-section measurement for different angle of muons as a function kinetic energy of muon. The data (statistical + systematic uncertainty) is shown in black and GENIE (NOvA tuned) prediction is shown in red and the bottom plot shows the ratio of data and other generator. In the bottom plot, the colors (for other generator) are divided from data points (black). The NEUT predictions show large statistical uncertainties, which are due to low statistics. The numerical value are quoted in the appendix. 


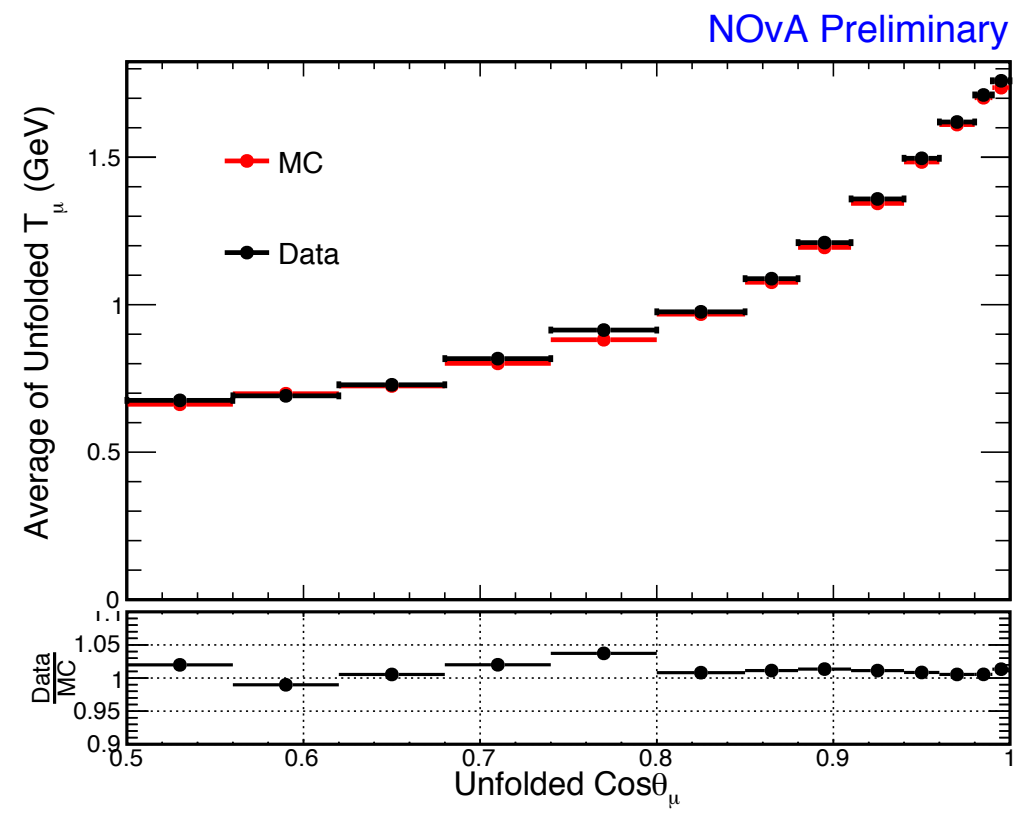

Figure 7.3: Average of unfolded kinetic energy of muon is plotted against different cosine angle of muons. The data is shown in black and GENIE MC (GENIE (NOvA tuned)) prediction is shown in red and the bottom plot shows the ratio of data and MC (GENIE (NOvA tuned)).

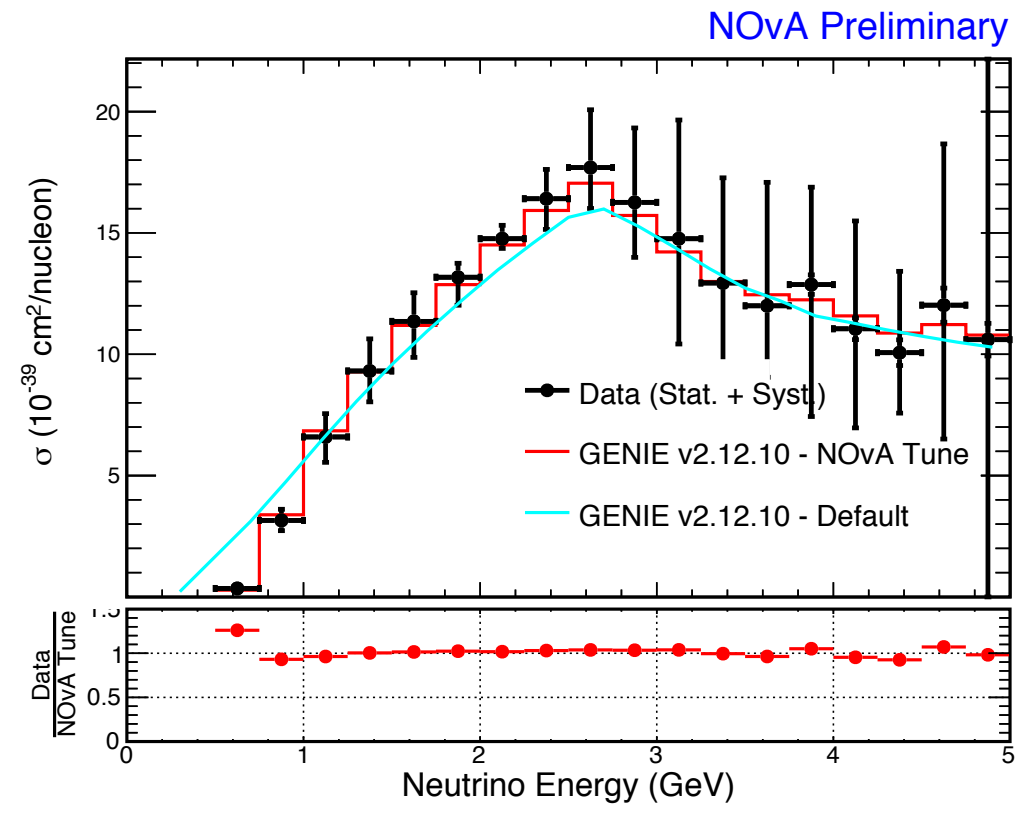

Figure 7.4: The kinematically constrained, measured total cross-section as a function of neutrino energy. The data (statistical + systematic uncertainty) is shown in black and GENIE (NOvA tuned) prediction is shown in red and the bottom plot shows the ratio of data and GENIE (NOvA tuned) and cyan color represents the prediction from GENIE(default) generator. The numerical value are quoted in the appendix. 
The inclusive measurement, in this energy region, is very relevant for current and future oscillation measurements.

The data used for this measurement consist of $8.09 \times 10^{20}$ POT of neutrino beam collected between August 2014 and February 2017.

The analysis strategy presented in this thesis was successful in measurement of the double differential (flux averaged) cross-section using muon neutrino beam. In the near future, this measurement can be improved including beam focusing and muon energy scale systematics which has not been added in this dissertation.

Neutrino oscillation physics is moving into a precision era and the most dominant uncertainties on oscillation parameters arise from our limited understanding of neutrino cross-section. The accuracy of these measurements depends explicitly on how well we are able to measure the energy of the incoming neutrino. The neutrino energy is estimated from the lepton and hadrons visible in the final state, after the neutrino has interacted. To relate these final state observables to the energy of the neutrino, accurate knowledge of neutrino-nucleon interaction cross sections and the dynamics of the propagation of particles through the nuclear matter are necessary.

This systematics-limited measurement is of interest to the general neutrino community, and the measured kinematic distributions of the final-state muon can be used to improve the simulation of neutrino interactions in the NOvA detectors as well as other long-baseline neutrino oscillation experiments. Additionally, this measurement may serve as a basis for future muon-neutrino semi-inclusive cross section measurements in NOvA, in particular, where the dominant flux systematic uncertainty can be mitigated via the ratio of semi-inclusive to inclusive measurements. 


\section{Appendix}

\begin{tabular}{|c|c|c|c|c|}
\hline $\cos \theta_{\mu}$ & $T_{\mu}$ & $\frac{d^{2} \sigma}{d \cos \theta_{\mu} d T_{\mu}}\left(10^{-39} \mathrm{~cm}^{2}\right)$ & Stat Unc. $(\%)$ & Syst Unc. $( \pm 1 \sigma)(\%)$ \\
\hline & {$[0.5,0.6)$} & 3.39 & 0.031 & ${ }_{-0.47}^{+0.4}$ \\
\hline & $(0.6,0.7)$ & 4.9 & 0.016 & $\begin{array}{l}+0.45 \\
{ }_{-0.98}\end{array}$ \\
\hline & $(0.7,0.8)$ & 7.36 & 0.014 & $\begin{array}{l}+0.94 \\
-0.82\end{array}$ \\
\hline & $(0.8,0.9)$ & 9.86 & 0.012 & $\begin{array}{l}+1.1 \\
-1.5\end{array}$ \\
\hline & $(0.9,1)$ & 13.5 & 0.01 & $\begin{array}{l}+0.8 \\
-0.97\end{array}$ \\
\hline & $(1,1.1)$ & 19.4 & 0.0094 & ${ }_{-0.71}^{+0.54}$ \\
\hline & $(1.1,1.2)$ & 25.5 & 0.0082 & $\begin{array}{l}+0.58 \\
-0.61\end{array}$ \\
\hline & $(1.2,1.3)$ & 32 & 0.0076 & ${ }_{-0.34}^{+0.33}$ \\
\hline & $(1.3,1.4)$ & 39.1 & 0.0071 & ${ }_{-0.33}^{+0.3}$ \\
\hline$(0.99,1]$ & $(1.4,1.5)$ & 45.9 & 0.0066 & $\begin{array}{l}+0.76 \\
{ }_{-0.64}\end{array}$ \\
\hline & $(1.5,1.6)$ & 52.6 & 0.0063 & ${ }_{-0.56}^{+0.55}$ \\
\hline & $(1.6,1.7)$ & 56.8 & 0.0061 & ${ }_{-0.3}^{+0.28}$ \\
\hline & $(1.7,1.8)$ & 60.9 & 0.0062 & $\begin{array}{l}+0.39 \\
-0.31\end{array}$ \\
\hline & $(1.8,1.9)$ & 60.6 & 0.0062 & $\begin{array}{l}+0.63 \\
-0.47\end{array}$ \\
\hline & $(1.9,2)$ & 58.3 & 0.0065 & ${ }_{-0.55}^{+0.4}$ \\
\hline & $(2,2.1)$ & 57 & 0.0069 & ${ }_{-0.34}^{+0.6}$ \\
\hline & $(2.1,2.2)$ & 53.7 & 0.0075 & $\begin{array}{l}+0.39 \\
-0.28\end{array}$ \\
\hline & $(2.2,2.3)$ & 48.4 & 0.0083 & $\begin{array}{l}+0.38 \\
-0.46\end{array}$ \\
\hline & $(2.3,2.4)$ & 42.9 & 0.0092 & $\begin{array}{l}+0.39 \\
-0.51\end{array}$ \\
\hline & $(2.4,2.5]$ & 40.3 & 0.011 & ${ }_{-0.35}^{+0.79}$ \\
\hline
\end{tabular}

Table 7.1: For $\cos \theta_{\mu}(0.99-1.0)$ bin, the measured double-differential cross section as a function of $T_{\mu}$ and its uncertainties. 


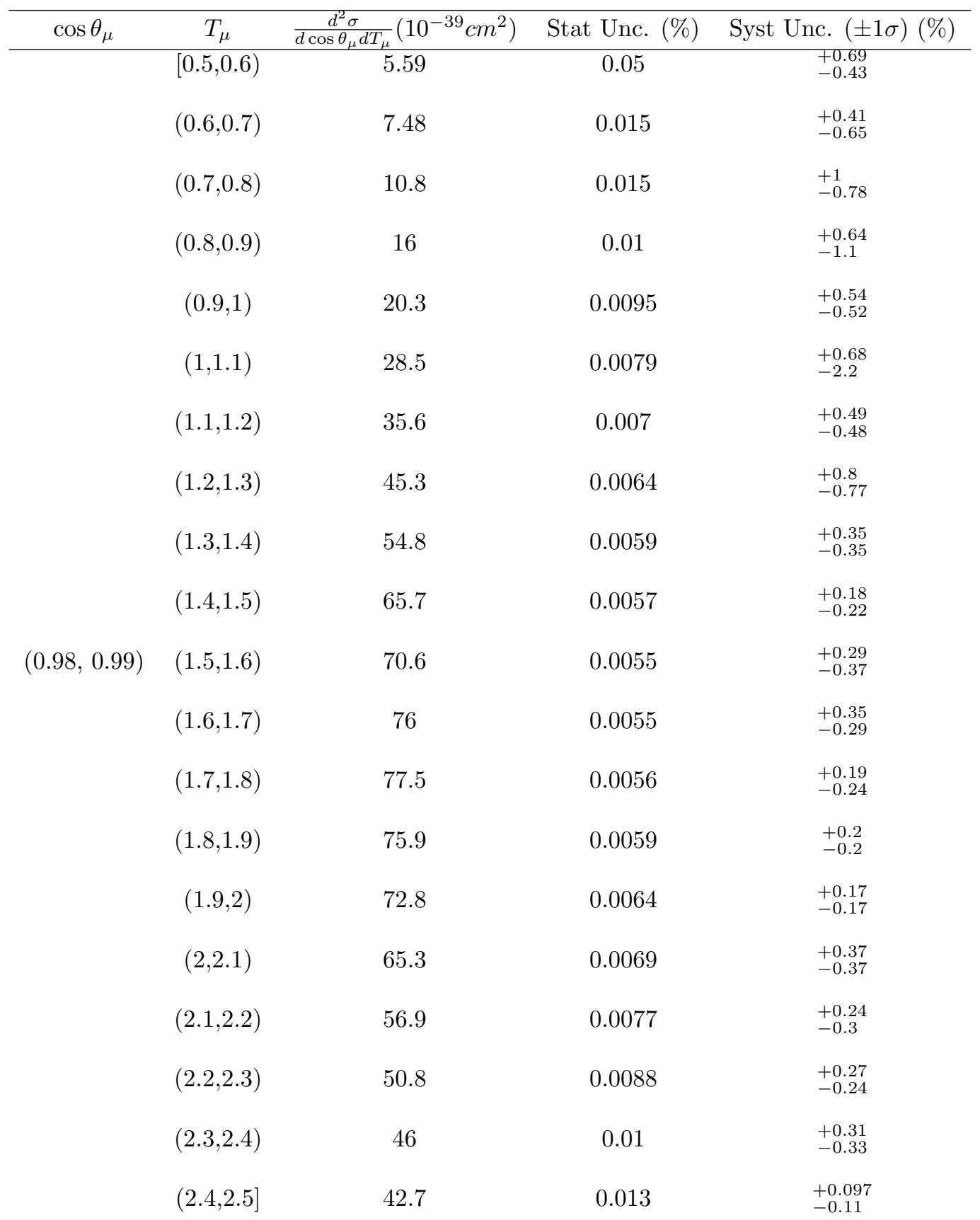

Table 7.2: For $\cos \theta_{\mu}(0.98$ - 0.99) bin, the measured double-differential cross section as a function of $T_{\mu}$ and its uncertainties. 


\begin{tabular}{|c|c|c|c|c|}
\hline $\cos \theta_{\mu}$ & $T_{\mu}$ & $\frac{d^{2} \sigma}{d \cos \theta_{\mu} d T_{\mu}}\left(10^{-39} \mathrm{~cm}^{2}\right)$ & Stat Unc. $(\%)$ & Syst Unc. $( \pm 1 \sigma)(\%)$ \\
\hline & {$[0.5,0.6)$} & 6.82 & 0.012 & $\begin{array}{l}+0.74 \\
{ }_{-0.9}\end{array}$ \\
\hline & $(0.6,0.7)$ & 10.5 & 0.0089 & $\begin{array}{l}+0.74 \\
-1.8\end{array}$ \\
\hline & $(0.7,0.8)$ & 13.9 & 0.0076 & $\begin{array}{l}+0.98 \\
-0.94\end{array}$ \\
\hline & $(0.8,0.9)$ & 19.9 & 0.0064 & $\begin{array}{l}+1.6 \\
-0.86\end{array}$ \\
\hline & $(0.9,1)$ & 27.7 & 0.0056 & $\begin{array}{l}+1.1 \\
{ }_{-0.98}\end{array}$ \\
\hline & $(1,1.1)$ & 35.3 & 0.0051 & ${ }_{-1.1}^{+1.7}$ \\
\hline & $(1.1,1.2)$ & 44.7 & 0.0047 & $\begin{array}{l}+0.98 \\
{ }_{-1.5}\end{array}$ \\
\hline & $(1.2,1.3)$ & 54.7 & 0.0045 & $\begin{array}{l}+0.53 \\
-0.51\end{array}$ \\
\hline & $(1.3,1.4)$ & 62.9 & 0.0043 & $\begin{array}{l}+0.3 \\
-0.34\end{array}$ \\
\hline & $(1.4,1.5)$ & 69.9 & 0.0042 & $\begin{array}{l}+0.35 \\
-0.6\end{array}$ \\
\hline \multirow[t]{10}{*}{$(0.96,0.98)$} & $(1.5,1.6)$ & 74.7 & 0.0043 & $\begin{array}{l}+0.92 \\
-0.53\end{array}$ \\
\hline & $(1.6,1.7)$ & 74.9 & 0.0045 & $\begin{array}{l}+0.56 \\
{ }_{-0.49}\end{array}$ \\
\hline & $(1.7,1.8)$ & 73.9 & 0.0048 & ${ }_{-0.2}^{+0.24}$ \\
\hline & $(1.8,1.9)$ & 67.5 & 0.0052 & ${ }_{-0.64}^{+0.4}$ \\
\hline & $(1.9,2)$ & 60.4 & 0.006 & $\begin{array}{l}+0.82 \\
{ }_{-0.48}\end{array}$ \\
\hline & $(2,2.1)$ & 51.9 & 0.0068 & $\begin{array}{l}+0.35 \\
{ }_{-0.43}\end{array}$ \\
\hline & $(2.1,2.2)$ & 42.9 & 0.0081 & $\begin{array}{l}+0.34 \\
{ }_{-0.38}\end{array}$ \\
\hline & $(2.2,2.3)$ & 36.2 & 0.0098 & $\begin{array}{l}+0.31 \\
-0.26\end{array}$ \\
\hline & $(2.3,2.4)$ & 29.9 & 0.012 & $\begin{array}{l}+0.16 \\
{ }_{-0.16}\end{array}$ \\
\hline & $(2.4,2.5]$ & 25 & 0.016 & ${ }_{-0.14}^{+0.14}$ \\
\hline
\end{tabular}

Table 7.3: For $\cos \theta_{\mu}(0.96$ - 0.98) bin, the measured double-differential cross section as a function of $T_{\mu}$ and its uncertainties. 


\begin{tabular}{|c|c|c|c|c|}
\hline $\cos \theta_{\mu}$ & $T_{\mu}$ & $\frac{d^{2} \sigma}{d \cos \theta_{\mu} d T_{\mu}}\left(10^{-39} \mathrm{~cm}^{2}\right)$ & Stat Unc. $(\%)$ & Syst Unc. $( \pm 1 \sigma)(\%)$ \\
\hline & {$[0.5,0.6)$} & 9.78 & 0.01 & $\begin{array}{l}+0.57 \\
{ }_{-0.16}\end{array}$ \\
\hline & $(0.6,0.7)$ & 13.9 & 0.0083 & $\begin{array}{l}+0.86 \\
{ }_{-0.58}\end{array}$ \\
\hline & $(0.7,0.8)$ & 18.6 & 0.0069 & $\begin{array}{l}+0.8 \\
-0.84\end{array}$ \\
\hline & $(0.8,0.9)$ & 23.9 & 0.0061 & ${ }_{-0.73}^{+0.86}$ \\
\hline & $(0.9,1)$ & 31.4 & 0.0056 & $\begin{array}{l}+0.58 \\
{ }_{-0.67}\end{array}$ \\
\hline & $(1,1.1)$ & 40.3 & 0.0053 & ${ }_{-0.91}^{+0.93}$ \\
\hline & $(1.1,1.2)$ & 48.1 & 0.0054 & $\begin{array}{l}+1.8 \\
{ }_{-0.51}\end{array}$ \\
\hline & $(1.2,1.3)$ & 56.3 & 0.0049 & ${ }_{-0.31}^{+0.28}$ \\
\hline & $(1.3,1.4)$ & 61 & 0.005 & $\begin{array}{l}+0.43 \\
{ }_{-0.55}\end{array}$ \\
\hline & $(1.4,1.5)$ & 64.9 & 0.0053 & $\begin{array}{l}+0.33 \\
{ }_{-0.39}\end{array}$ \\
\hline \multirow[t]{10}{*}{$(0.94,0.96)$} & $(1.5,1.6)$ & 62.9 & 0.0057 & $\begin{array}{l}+0.58 \\
{ }_{-0.84}\end{array}$ \\
\hline & $(1.6,1.7)$ & 59.3 & 0.0065 & ${ }_{-0.34}^{+0.32}$ \\
\hline & $(1.7,1.8)$ & 53.6 & 0.0075 & $\begin{array}{l}+0.58 \\
{ }_{-0.99}\end{array}$ \\
\hline & $(1.8,1.9)$ & 46.8 & 0.009 & ${ }_{-0.4}^{+0.39}$ \\
\hline & $(1.9,2)$ & 37.9 & 0.011 & $\begin{array}{l}+0.33 \\
-0.46\end{array}$ \\
\hline & $(2,2.1)$ & 30.4 & 0.014 & ${ }_{-0.26}^{+0.23}$ \\
\hline & $(2.1,2.2)$ & 23.1 & 0.018 & ${ }_{-0.64}^{+0.31}$ \\
\hline & $(2.2,2.3)$ & 17.4 & 0.026 & ${ }_{-0.29}^{+0.26}$ \\
\hline & $(2.3,2.4)$ & 13.9 & 0.033 & ${ }_{-0.39}^{+0.26}$ \\
\hline & $(2.4,2.5]$ & 11.1 & 0.047 & +0.18 \\
\hline
\end{tabular}

Table 7.4: For $\cos \theta_{\mu}(0.94-0.96)$ bin, the measured double-differential cross section as a function of $T_{\mu}$ and its uncertainties. 


\begin{tabular}{|c|c|c|c|c|}
\hline $\cos \theta_{\mu}$ & $T_{\mu}$ & $\frac{d^{2} \sigma}{d \cos \theta_{\mu} d T_{\mu}}\left(10^{-39} \mathrm{~cm}^{2}\right)$ & Stat Unc. (\%) & Syst Unc. $( \pm 1 \sigma)(\%)$ \\
\hline & {$[0.5,0.6)$} & 11.6 & 0.0075 & ${ }_{-1.2}^{+0.71}$ \\
\hline & $(0.6,0.7)$ & 15.7 & 0.0063 & $\begin{array}{l}+1.2 \\
-1.1\end{array}$ \\
\hline & $(0.7,0.8)$ & 21.5 & 0.0056 & ${ }_{-1.6}^{+1.2}$ \\
\hline & $(0.8,0.9)$ & 27.4 & 0.0051 & $\begin{array}{l}+0.8 \\
-1.1\end{array}$ \\
\hline & $(0.9,1)$ & 34.8 & 0.0049 & $\begin{array}{l}+0.84 \\
-0.66\end{array}$ \\
\hline & $(1,1.1)$ & 41.5 & 0.0048 & $\begin{array}{l}+1.4 \\
{ }_{-0.94}\end{array}$ \\
\hline & $(1.1,1.2)$ & 47.5 & 0.0048 & ${ }_{-1}^{+1.3}$ \\
\hline & $(1.2,1.3)$ & 51.1 & 0.0051 & $\begin{array}{l}+0.72 \\
-0.34\end{array}$ \\
\hline & $(1.3,1.4)$ & 52.9 & 0.0057 & ${ }_{-0.6}^{+0.75}$ \\
\hline & $(1.4,1.5)$ & 51.9 & 0.0066 & $\begin{array}{l}+0.34 \\
{ }_{-0.38}\end{array}$ \\
\hline \multirow[t]{9}{*}{$(0.91,0.94)$} & $(1.5,1.6)$ & 47.5 & 0.0078 & $\begin{array}{l}+0.28 \\
{ }_{-0.2}\end{array}$ \\
\hline & $(1.6,1.7)$ & 40.3 & 0.0098 & ${ }_{-0.23}^{+0.22}$ \\
\hline & $(1.7,1.8)$ & 33.6 & 0.013 & $\begin{array}{l}+0.3 \\
-0.58\end{array}$ \\
\hline & $(1.8,1.9)$ & 25.5 & 0.016 & ${ }_{-0.21}^{+0.27}$ \\
\hline & $(1.9,2)$ & 17.8 & 0.023 & ${ }_{-0.18}^{+0.17}$ \\
\hline & $(2,2.1)$ & 12.6 & 0.029 & ${ }_{-0.17}^{+0.19}$ \\
\hline & $(2.1,2.2)$ & 9.61 & 0.041 & ${ }_{-0.23}^{+0.31}$ \\
\hline & $(2.2,2.3)$ & 7.22 & 0.06 & $\begin{array}{l}+0.08 \\
{ }_{-0.22}\end{array}$ \\
\hline & $(2.3,2.4)$ & 4.82 & 0.12 & ${ }_{-0.17}^{+0.17}$ \\
\hline
\end{tabular}

Table 7.5: For $\cos \theta_{\mu}(0.91-0.94)$ bin, the measured double-differential cross section as a function of $T_{\mu}$ and its uncertainties. 


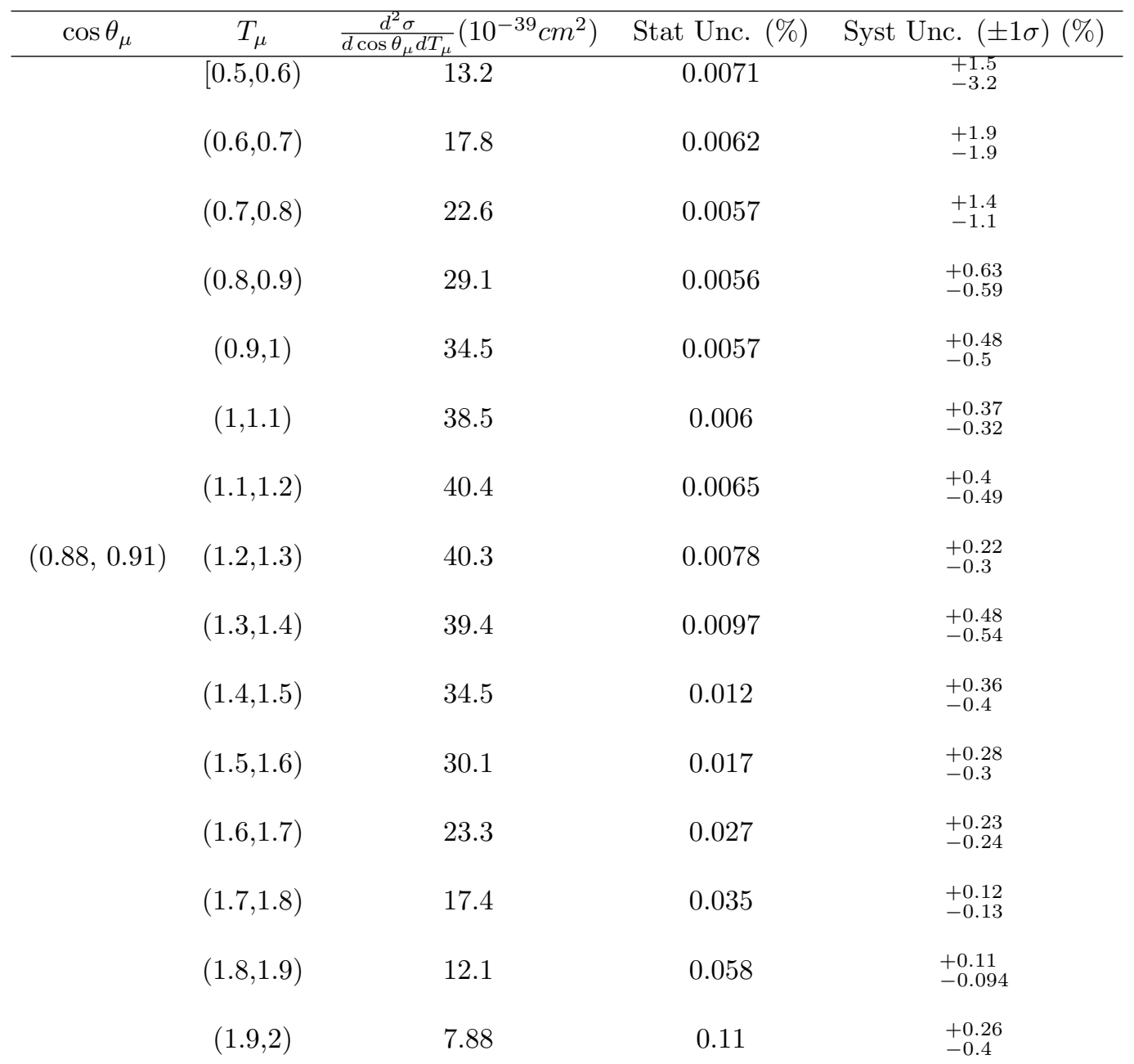

Table 7.6: For $\cos \theta_{\mu}(0.88-0.91)$ bin, the measured double-differential cross section as a function of $T_{\mu}$ and its uncertainties. 


\begin{tabular}{|c|c|c|c|c|}
\hline $\cos \theta_{\mu}$ & $T_{\mu}$ & $\frac{d^{2} \sigma}{d \cos \theta_{\mu} d T_{\mu}}\left(10^{-39} \mathrm{~cm}^{2}\right)$ & Stat Unc. $(\%)$ & Syst Unc. $( \pm 1 \sigma)(\%)$ \\
\hline & {$[0.5,0.6)$} & 14.1 & 0.0071 & $\begin{array}{l}+1.2 \\
-1.5\end{array}$ \\
\hline & $(0.6,0.7)$ & 18 & 0.0063 & $\begin{array}{l}+1.7 \\
-1.5\end{array}$ \\
\hline & $(0.7,0.8)$ & 23 & 0.0062 & $\begin{array}{l}+0.8 \\
-0.88\end{array}$ \\
\hline & $(0.8,0.9)$ & 29 & 0.0063 & $\begin{array}{l}+0.32 \\
-0.32\end{array}$ \\
\hline & $(0.9,1)$ & 31.4 & 0.0068 & $\begin{array}{l}+1.4 \\
-0.89\end{array}$ \\
\hline & $(1,1.1)$ & 31.9 & 0.0077 & $\begin{array}{l}+0.75 \\
-0.44\end{array}$ \\
\hline \multirow[t]{6}{*}{$(0.85,0.88)$} & $(1.1,1.2)$ & 34.2 & 0.0095 & $\begin{array}{l}+0.6 \\
-0.63\end{array}$ \\
\hline & $(1.2,1.3)$ & 33.3 & 0.013 & $\begin{array}{l}+0.26 \\
-0.26\end{array}$ \\
\hline & $(1.3,1.4)$ & 28.2 & 0.02 & $\begin{array}{l}+0.25 \\
-0.26\end{array}$ \\
\hline & $(1.4,1.5)$ & 24 & 0.036 & $\begin{array}{l}+0.27 \\
-0.28\end{array}$ \\
\hline & $(1.5,1.6)$ & 15.3 & 0.05 & $\begin{array}{l}+0.22 \\
-0.21\end{array}$ \\
\hline & $(1.6,1.7)$ & 11.5 & 0.094 & $\begin{array}{l}+0.22 \\
-0.21\end{array}$ \\
\hline
\end{tabular}

Table 7.7: For $\cos \theta_{\mu}(0.85-0.88)$ bin, the measured double-differential cross section as a function of $T_{\mu}$ and its uncertainties. 


\begin{tabular}{ccccc}
\hline $\cos \theta_{\mu}$ & $T_{\mu}$ & $\frac{d^{2} \sigma}{d \cos \theta_{\mu} d T_{\mu}}\left(10^{-39} \mathrm{~cm}^{2}\right)$ & Stat Unc. (\%) & Syst Unc. $( \pm 1 \sigma)(\%)$ \\
\hline & $10.5,0.6)$ & 15.3 & 0.0056 & ${ }_{-0.54}^{+0.85}$ \\
& $(0.6,0.7)$ & 18.5 & 0.0053 & ${ }_{-1.4}^{+0.98}$ \\
& & & & \\
& $(0.7,0.8)$ & 22.6 & 0.0054 & ${ }_{-0.81}^{+0.93}$ \\
& $(0.8,0.9)$ & 26 & 0.0059 & ${ }_{-2.2}^{+0.99}$ \\
& $(0.9,1)$ & 27.6 & 0.0071 & ${ }_{-1.1}^{+1.1}$ \\
$(0.80,0.85)$ & $(1,1.1)$ & 26.1 & 0.009 & ${ }_{-0.67}^{+0.56}$ \\
& & & & \\
& $(1.1,1.2)$ & 23.2 & 0.013 & ${ }_{-0.95}^{+0.85}$ \\
& & & & \\
& $(1.2,1.3)$ & 20.1 & 0.021 & ${ }_{-0.3}^{+0.51}$ \\
& $(1.3,1.4)$ & 16.3 & 0.035 & ${ }_{-0.27}^{+0.27}$ \\
& $(1.4,1.5)$ & 8.78 & 0.07 & ${ }_{-0.15}^{+0.28}$
\end{tabular}

Table 7.8: For $\cos \theta_{\mu}(0.80-0.85)$ bin, the measured double-differential cross section as a function of $T_{\mu}$ and its uncertainties.

\begin{tabular}{ccccc}
\hline $\cos \theta_{\mu}$ & $T_{\mu}$ & $\frac{d^{2} \sigma}{d \cos \theta_{\mu} d T_{\mu}}\left(10^{-39} \mathrm{~cm}^{2}\right)$ & Stat Unc. (\%) & Syst Unc. $( \pm 1 \sigma)(\%)$ \\
\hline & $(0.5,0.6)$ & 14.7 & 0.0055 & ${ }_{-0.76}^{+0.94}$ \\
& $(0.6,0.7)$ & 18.4 & 0.0056 & ${ }_{-1.7}^{+3}$ \\
& $(0.7,0.8)$ & 19.4 & 0.0062 & ${ }_{-0.79}^{+0.6}$ \\
& $(0.8,0.9)$ & 20 & 0.0076 & ${ }_{-0.9}^{+0.82}$ \\
$(0.74,0.80)$ & $(0.9,1)$ & 19.9 & 0.011 & ${ }_{-0.83}^{+0.61}$ \\
& $(1,1.1)$ & 16.5 & 0.017 & ${ }_{-0.63}^{+0.18}$ \\
& $(1.1,1.2)$ & 14.9 & 0.034 & ${ }_{-0.37}^{+0.52}$ \\
& $(1.2,1.3)$ & 10.7 & 0.068 & ${ }_{-0.23}^{+0.2}$ \\
& & & & \\
& & & & \\
& & & & \\
-0.0066
\end{tabular}

Table 7.9: For $\cos \theta_{\mu}(0.74-0.80)$ bin, the measured double-differential cross section as a function of $T_{\mu}$ and its uncertainties. 


\begin{tabular}{ccccc}
\hline $\cos \theta_{\mu}$ & $T_{\mu}$ & $\frac{d^{2} \sigma}{d \cos \theta_{\mu} d T_{\mu}}\left(10^{-39} \mathrm{~cm}^{2}\right)$ & Stat Unc. (\%) & Syst Unc. $( \pm 1 \sigma)(\%)$ \\
\hline & {$[0.5,0.6)$} & 13.9 & 0.0063 & ${ }_{-1.3}^{+1.7}$ \\
& $(0.6,0.7)$ & 16.4 & 0.0068 & ${ }_{-1.1}^{+1}$ \\
& $(0.7,0.8)$ & 17.4 & 0.0081 & ${ }_{-0.79}^{+0.8}$ \\
$(0.68,0.74)$ & $(0.8,0.9)$ & 15.5 & 0.012 & ${ }_{-0.47}^{+0.7}$ \\
& $(0.9,1)$ & 14.5 & 0.021 & ${ }_{-0.47}^{+0.21}$ \\
& $(1,1.1)$ & 7.54 & 0.046 & ${ }_{-0.3}^{+0.56}$ \\
& & & & ${ }_{-0.1}^{+0.094}$
\end{tabular}

Table 7.10: For $\cos \theta_{\mu}(0.68-0.74)$ bin, the measured double-differential cross section as a function of $T_{\mu}$ and its uncertainties.

\begin{tabular}{ccccc}
\hline $\cos \theta_{\mu}$ & $T_{\mu}$ & $\frac{d^{2} \sigma}{d \cos \theta_{\mu} d T_{\mu}}\left(10^{-39} \mathrm{~cm}^{2}\right)$ & Stat Unc. (\%) & Syst Unc. $( \pm 1 \sigma)(\%)$ \\
\hline & {$[0.5,0.6)$} & 13.4 & 0.0073 & ${ }_{-0.72}^{+1.7}$ \\
& $(0.6,0.7)$ & 14.4 & 0.0084 & ${ }_{-0.93}^{+1.4}$ \\
$(0.62,0.68)$ & $(0.7,0.8)$ & 12.8 & 0.011 & ${ }_{-0.55}^{+0.52}$ \\
& $(0.8,0.9)$ & 11.6 & 0.02 & ${ }_{-0.45}^{+0.56}$ \\
& & & & \\
& $(0.9,1)$ & 8.61 & 0.043 & ${ }_{-0.35}^{+0.29}$
\end{tabular}

Table 7.11: For $\cos \theta_{\mu}(0.62-0.68)$ bin, the measured double-differential cross section as a function of $T_{\mu}$ and its uncertainties.

\begin{tabular}{ccccc}
\hline $\cos \theta_{\mu}$ & $T_{\mu}$ & $\frac{d^{2} \sigma}{d \cos \theta_{\mu} d T_{\mu}}\left(10^{-39} \mathrm{~cm}^{2}\right)$ & Stat Unc. (\%) & Syst Unc. $( \pm 1 \sigma)(\%)$ \\
\hline & {$[0.5,0.6)$} & 11.5 & 0.0085 & ${ }_{-2}^{+1.7}$ \\
& $(0.6,0.7)$ & 11.2 & 0.01 & ${ }_{-0.54}^{+0.61}$ \\
$(0.56,0.62)$ & $(0.7,0.8)$ & 9.97 & 0.016 & ${ }_{-0.43}^{+0.4}$ \\
& $(0.8,0.9)$ & 6.97 & 0.034 & ${ }_{-0.34}^{+0.31}$ \\
& & & & \\
& $(0.9,1)$ & 3.39 & 0.25 & ${ }_{-0.42}^{+0.53}$
\end{tabular}

Table 7.12: For $\cos \theta_{\mu}(0.56-0.62)$ bin, the measured double-differential cross section as a function of $T_{\mu}$ and its uncertainties. 


\begin{tabular}{ccccc}
\hline $\cos \theta_{\mu}$ & $T_{\mu}$ & $\frac{d^{2} \sigma}{d \cos \theta_{\mu} d T_{\mu}}\left(10^{-39} \mathrm{~cm}^{2}\right)$ & Stat Unc. (\%) & Syst Unc. $( \pm 1 \sigma)(\%)$ \\
\hline & {$[0.5,0.6)$} & 10.2 & 0.011 & ${ }_{-0.5}^{+0.81}$ \\
& $(0.6,0.7)$ & 9.47 & 0.014 & ${ }_{-0.99}^{+1}$ \\
$(0.5,0.56]$ & $(0.7,0.8)$ & 6.92 & 0.026 & ${ }_{-0.33}^{+0.39}$ \\
& $(0.8,0.9)$ & 5.54 & 0.065 & ${ }_{-0.37}^{+0.42}$
\end{tabular}

Table 7.13: For $\cos \theta_{\mu}(0.5-0.56)$ bin, the measured double-differential cross section as a function of $T_{\mu}$ and its uncertainties. 


\begin{tabular}{|c|c|c|c|}
\hline$E_{\nu}$ & $\sigma\left(10^{-39} \mathrm{~cm}^{2}\right)$ & Stat Unc. $(\%)$ & Syst Unc. $( \pm 1 \sigma)(\%)$ \\
\hline$[0.5,0.75)$ & 0.349 & 0.016 & ${ }_{-8.1}^{+11}$ \\
\hline$(0.75,1)$ & 3.15 & 0.0048 & ${ }_{-2.4}^{+2.2}$ \\
\hline$(1,1.2)$ & 6.59 & 0.0033 & ${ }_{-0.96}^{+1}$ \\
\hline$(1.2,1.5)$ & 9.31 & 0.0026 & $\begin{array}{l}+0.76 \\
-0.79\end{array}$ \\
\hline$(1.5,1.8)$ & 11.4 & 0.0026 & ${ }_{-0.67}^{+0.85}$ \\
\hline$(1.8,2)$ & 13.2 & 0.0042 & $\begin{array}{l}+1.7 \\
-0.87\end{array}$ \\
\hline$(2,2.2)$ & 14.8 & 0.0037 & ${ }_{-2.5}^{+1.8}$ \\
\hline$(2.2,2.5)$ & 16.4 & 0.0059 & $\begin{array}{l}+0.83 \\
{ }_{-0.79}\end{array}$ \\
\hline$(2.5,2.8)$ & 17.7 & 0.0056 & $\begin{array}{l}+0.42 \\
{ }_{-0.59}\end{array}$ \\
\hline$(2.8,3)$ & 16.3 & 0.0075 & $\begin{array}{l}+0.33 \\
-0.44\end{array}$ \\
\hline$(3,3.2)$ & 14.8 & 0.015 & $\begin{array}{l}+0.2 \\
-0.23\end{array}$ \\
\hline$(3.2,3.5)$ & 12.9 & 0.019 & $\begin{array}{l}+0.23 \\
-0.25\end{array}$ \\
\hline$(3.5,3.8)$ & 12 & 0.02 & ${ }_{-0.34}^{+0.2}$ \\
\hline$(3.8,4)$ & 12.9 & 0.031 & $\begin{array}{l}+0.25 \\
-0.18\end{array}$ \\
\hline$(4,4.2)$ & 11.1 & 0.04 & ${ }_{-0.24}^{+0.22}$ \\
\hline$(4.2,4.5)$ & 10.1 & 0.053 & ${ }_{-0.4}^{+0.3}$ \\
\hline$(4.5,4.8)$ & 12 & 0.059 & $\begin{array}{l}+0.15 \\
-0.18\end{array}$ \\
\hline$(4.8,5]$ & 10.6 & 0.063 & ${ }_{-0.071}^{+0.072}$ \\
\hline
\end{tabular}

Table 7.14: The cross-section measurements as a function of neutrino energy derived from the double-differential results and its uncertainties. 


\section{References}

[1] S. L. Glashow. Partial Symmetries of Weak Interactions. Nucl. Phys. 22, (1961) 579-588.

[2] A. Salam. Weak and Electromagnetic Interactions. In N. Svartholm, ed., Elementary particle theory. Almquist \& Wiksell 367-377.

[3] S. Weinberg. A Model of Leptons. Phys. Rev. Lett. 19, (1967) 1264-1266.

[4] F. Englert and R. Brout. Broken Symmetry and the Mass of Gauge Vector Mesons. Phys. Rev. Lett. 13, (1964) 321-323.

[5] P. W. Higgs. Broken symmetries, massless particles and gauge fields. Phys. Lett. 12, (1964) $132-133$.

[6] P. W. Higgs. Broken Symmetries and the Masses of Gauge Bosons. Phys. Rev. Lett. 13, (1964) 508-509.

[7] G. S. Guralnik, C. R. Hagen, and T. W. B. Kibble. Global Conservation Laws and Massless Particles. Phys. Rev. Lett. 13, (1964) 585-587.

[8] G. Aad et al. Observation of a new particle in the search for the Standard Model Higgs boson with the ATLAS detector at the LHC. Phys. Lett. B716, (2012) 1-29.

[9] S. Chatrchyan et al. Observation of a new boson at a mass of $125 \mathrm{GeV}$ with the CMS experiment at the LHC. Phys. Lett. B716, (2012) 30-61.

[10] W. Pauli. Dear radioactive ladies and gentlemen. Phys. Today 31N9, (1978) 27.

[11] J. Steinberger. On the Range of the Electrons in Meson Decay. Phys. Rev. 74, (1948) 500-501.

[12] J. Chadwick. Possible Existence of a Neutron. Nature 129, (1932) 312.

[13] E. Fermi. An attempt of a theory of beta radiation. 1. Z. Phys. 88, (1934) 161-177.

[14] E. Fermi. Trends to a Theory of beta Radiation. (In Italian). Nuovo Cim. 11, (1934) 1-19. $[, 535(1934)]$.

[15] F. Reines and C. L. Cowan. Detection of the free neutrino. Phys. Rev. 92, (1953) 830-831.

[16] C. L. Cowan, F. Reines, F. B. Harrison, H. W. Kruse, and A. D. McGuire. Detection of the free neutrino: A Confirmation. Science 124, (1956) 103-104. 
[17] F. Reines and C. L. Cowan. Free anti-neutrino absorption cross-section. 1: Measurement of the free anti-neutrino absorption cross-section by protons. Phys. Rev. 113, (1959) 273-279.

[18] F. Reines, C. L. Cowan, F. B. Harrison, A. D. McGuire, and H. W. Kruse. Detection of the free anti-neutrino. Phys. Rev. 117, (1960) 159-173.

[19] B. Pontecorvo. Mesonium and anti-mesonium. Sov. Phys. JETP 6, (1957) 429. [Zh. Eksp. Teor. Fiz.33,549(1957)].

[20] G. Danby, J. M. Gaillard, K. A. Goulianos, L. M. Lederman, N. B. Mistry, M. Schwartz, and J. Steinberger. Observation of High-Energy Neutrino Reactions and the Existence of Two Kinds of Neutrinos. Phys. Rev. Lett. 9, (1962) 36-44.

[21] Z. Maki, M. Nakagawa, and S. Sakata. Remarks on the unified model of elementary particles. Prog. Theor. Phys. 28, (1962) 870-880. [,34(1962)].

[22] K. Kodama et al. Observation of tau neutrino interactions. Phys. Lett. B504, (2001) 218-224.

[23] L. Wolfenstein. Neutrino Oscillations in Matter. Phys. Rev. D17, (1978) 2369-2374. [,294(1977)].

[24] C. H. Llewellyn Smith. Neutrino Reactions at Accelerator Energies. Phys. Rept. 3, (1972) 261-379.

[25] S. S. Gershtein and Ya. B. Zeldovich. Meson corrections in the theory of beta decay. Zh. Eksp. Teor. Fiz. 29, (1955) 698-699. [,80(1955)].

[26] S. L. Glashow, J. Iliopoulos, and L. Maiani. Weak Interactions with Lepton-Hadron Symmetry. Phys. Rev. D2, (1970) 1285-1292.

[27] A. Bodek, S. Avvakumov, R. Bradford, and H. S. Budd. Extraction of the axial nucleon form-factor from neutrino experiments on deuterium. J. Phys. Conf. Ser. 110, (2008) 082,004.

[28] H. Gallagher, G. Garvey, and G. P. Zeller. Neutrino-nucleus interactions. Ann. Rev. Nucl. Part. Sci. 61, (2011) 355-378.

[29] R. P. Feynman, M. Kislinger, and F. Ravndal. Current matrix elements from a relativistic quark model. Phys. Rev. D3, (1971) 2706-2732.

[30] M. Glck, E. Reya, and A. Vogt. Dynamical parton distributions revisited. Eur. Phys. J. C5, (1998) 461-470.

[31] J. Delorme and M. Ericson. Exploration of the Spin - Isospin Nuclear Response Function by Neutrinos. Phys. Lett. 156B, (1985) 263-266.

[32] O. Lalakulich, U. Mosel, and K. Gallmeister. Energy reconstruction in quasielastic scattering in the MiniBooNE and T2K experiments. Phys. Rev. C86, (2012) 054,606.

[33] K. Gallmeister, U. Mosel, and J. Weil. Neutrino-Induced Reactions on Nuclei. Phys. Rev. C94, (2016) 035,502. 
[34] O. Lalakulich, K. Gallmeister, and U. Mosel. Many-Body Interactions of Neutrinos with Nuclei - Observables. Phys. Rev. C86, (2012) 014,614. [Erratum: Phys. Rev.C90,no.2,029902(2014)].

[35] U. Mosel, O. Lalakulich, and K. Gallmeister. Reaction mechanisms at MINERA. Phys. Rev. D89, (2014) 093,003.

[36] A. Bodek, H. S. Budd, and M. E. Christy. Neutrino Quasielastic Scattering on Nuclear Targets: Parametrizing Transverse Enhancement (Meson Exchange Currents). Eur. Phys. J. C71, (2011) 1726.

[37] M. Martini, M. Ericson, and G. Chanfray. Neutrino energy reconstruction problems and neutrino oscillations. Phys. Rev. D85, (2012) 093,012.

[38] M. Martini, M. Ericson, and G. Chanfray. Energy reconstruction effects in neutrino oscillation experiments and implications for the analysis. Phys. Rev. D87, (2013) 013,009.

[39] M. Martini, M. Ericson, G. Chanfray, and J. Marteau. Neutrino and antineutrino quasielastic interactions with nuclei. Phys. Rev. C81, (2010) 045,502.

[40] M. Martini, N. Jachowicz, M. Ericson, V. Pandey, T. Van Cuyck, and N. Van Dessel. Electronneutrino scattering off nuclei from two different theoretical perspectives. Phys. Rev. C94, (2016) 015,501 .

[41] M. Ericson and M. Martini. Neutrino versus antineutrino cross sections and $C P$ violation. Phys. Rev. C91, (2015) 035,501.

[42] J. Nieves, F. Sanchez, I. Ruiz Simo, and M. J. Vicente Vacas. Neutrino Energy Reconstruction and the Shape of the CCQE-like Total Cross Section. Phys. Rev. D85, (2012) 113,008.

[43] J. Nieves, I. Ruiz Simo, and M. J. Vicente Vacas. Inclusive Charged-Current Neutrino-Nucleus Reactions. Phys. Rev. C83, (2011) 045,501.

[44] J. Nieves, I. Ruiz Simo, and M. J. Vicente Vacas. The nucleon axial mass and the MiniBooNE Quasielastic Neutrino-Nucleus Scattering problem. Phys. Lett. B707, (2012) 72-75.

[45] J. Nieves, I. Ruiz Simo, and M. J. Vicente Vacas. Two Particle-Hole Excitations in Charged Current Quasielastic Antineutrino-Nucleus Scattering. Phys. Lett. B721, (2013) 90-93.

[46] R. Gran, J. Nieves, F. Sanchez, and M. J. Vicente Vacas. Neutrino-nucleus quasi-elastic and 2p2h interactions up to $10 \mathrm{GeV}$. Phys. Rev. D88, (2013) 113,007.

[47] J. E. Amaro, M. B. Barbaro, J. A. Caballero, T. W. Donnelly, and C. F. Williamson. Mesonexchange currents and quasielastic neutrino cross sections in the SuperScaling Approximation model. Phys. Lett. B696, (2011) 151-155.

[48] J. E. Amaro, M. B. Barbaro, J. A. Caballero, T. W. Donnelly, and J. M. Udias. Relativistic analyses of quasielastic neutrino cross sections at MiniBooNE kinematics. Phys. Rev. D84, (2011) 033,004. 
[49] J. E. Amaro, M. B. Barbaro, J. A. Caballero, and T. W. Donnelly. Meson-exchange currents and quasielastic antineutrino cross sections in the SuperScaling Approximation. Phys. Rev. Lett. 108, (2012) 152,501.

[50] I. Ruiz Simo, C. Albertus, J. E. Amaro, M. B. Barbaro, J. A. Caballero, and T. W. Donnelly. Relativistic effects in two-particle emission for electron and neutrino reactions. Phys. Rev. D90, (2014) 033,012.

[51] I. Ruiz Simo, C. Albertus, J. E. Amaro, M. B. Barbaro, J. A. Caballero, and T. W. Donnelly. Angular distribution in two-particle emission induced by neutrinos and electrons. Phys. Rev. D90, (2014) 053,010.

[52] G. D. Megias et al. Meson-exchange currents and quasielastic predictions for charged-current neutrino- ${ }^{12} C$ scattering in the superscaling approach. Phys. Rev. D91, (2015) 073,004.

[53] M. V. Ivanov, G. D. Megias, R. Gonzlez-Jimnez, O. Moreno, M. B. Barbaro, J. A. Caballero, and T. W. Donnelly. Charged-current inclusive neutrino cross sections in the SuperScaling model including quasielastic, pion production and meson-exchange contributions. J. Phys. G43, (2016) 045,101.

[54] I. Ruiz Simo, J. E. Amaro, M. B. Barbaro, A. De Pace, J. A. Caballero, and T. W. Donnelly. Relativistic model of $2 \mathrm{p}-2 \mathrm{~h}$ meson exchange currents in (anti)neutrino scattering. J. Phys. G44, (2017) 065,105.

[55] T. Golan. Modeling nuclear effects in NuWro Monte Carlo neutrino event generator. Ph.D. thesis, University of Wroclaw, Poland 2014.

[56] H. De Vries, C. W. De Jager, and C. De Vries. Nuclear charge and magnetization density distribution parameters from elastic electron scattering. Atom. Data Nucl. Data Tabl. 36, (1987) 495-536.

[57] C. Juszczak, J. A. Nowak, and J. T. Sobczyk. Spectrum of recoil nucleons in quasi-elastic neutrino nucleus interactions. Eur. Phys. J. C39, (2005) 195-200.

[58] R. Shneor et al. Investigation of proton-proton short-range correlations via the C-12(e, e-prime pp) reaction. Phys. Rev. Lett. 99, (2007) 072,501.

[59] M. M. Sargsian, T. V. Abrahamyan, M. I. Strikman, and L. L. Frankfurt. Exclusive electrodisintegration of He-3 at high Q2. II. Decay function formalism. Phys. Rev. C71, (2005) 044,615 .

[60] R. Schiavilla, R. B. Wiringa, S. C. Pieper, and J. Carlson. Tensor Forces and the Ground-State Structure of Nuclei. Phys. Rev. Lett. 98, (2007) 132,501.

[61] G. Co'. Random phase approximation and neutrino-nucleus cross sections. Acta Phys. Polon. B37, (2006) 2235-2242.

[62] R. Gran. Model Uncertainties for Valencia RPA Effect for MINERvA . 
[63] J.-E. Garca-Ramos, C. E. Alonso, M. V. Andrs, and F. Prez-Bernal. Proceedings, International Scientific Meeting on Nuclear Physics : Basic Concepts in Nuclear Physics: Theory, Experiments and Applications. (Rabida 15). Springer Proc. Phys. 182, (2016) pp.1-234.

[64] L. Alvarez-Ruso et al. NuSTEC 11 Neutrino Scattering Theory Experiment Collaboration http://nustec.fnal.gov . White Paper: Status and challenges of neutrinonucleus scattering. Prog. Part. Nucl. Phys. 100, (2018) 1-68.

[65] P. Adamson et al. The NuMI Neutrino Beam. Nucl. Instrum. Meth. A806, (2016) 279-306.

[66] D. S. Ayres et al. The NOvA Technical Design Report .

[67] R. M. Zwaska. Accelerator Systems and Instrumentation for the NuMI Neutrino Beam. Ph.D. thesis, Texas U. 2005.

[68] A. Norman. NOvA DAQ Data formats-Definitions. NOvA internal Note-4390 2009.

[69] E. Niner, P. Adamson, G. Deuerling, R. Kwarciany, H. Meyer, A. Norman, R. Rechenmacher, P. Shanahan, and N. Wilcer. Synchronization of the $14 \mathrm{kTon} \mathrm{NO} \nu \mathrm{A}$ neutrino detector with the Fermilab NuMI beam. J. Phys. Conf. Ser. 513, (2014) 012,028.

[70] S. Agostinelli et al. GEANT4: A Simulation toolkit. Nucl. Instrum. Meth. A506, (2003) 250-303.

[71] C. Andreopoulos et al. The GENIE Neutrino Monte Carlo Generator. Nucl. Instrum. Meth. A614, (2010) 87-104.

[72] R. Bradford, A. Bodek, H. S. Budd, and J. Arrington. A New parameterization of the nucleon elastic form-factors. Nucl. Phys. Proc. Suppl. 159, (2006) 127-132. [,127(2006)].

[73] D. Rein and L. M. Sehgal. Neutrino Excitation of Baryon Resonances and Single Pion Production. Annals Phys. 133, (1981) 79-153.

[74] H. Gallagher. The NEUGEN neutrino event generator. Nucl. Phys. Proc. Suppl. 112, (2002) 188-194. [,188(2002)].

[75] P. A. Cherenkov. Visible luminescence of pure liquids under the influence of -radiation. Dokl. Akad. Nauk SSSR 2, (1934) 451-454. [Usp. Fiz. Nauk93,no.2,385(1967)].

[76] I. M. Frank and I. E. Tamm. Coherent visible radiation of fast electrons passing through matter. Compt. Rend. Acad. Sci. URSS 14, (1937) 109-114. [Usp. Fiz. Nauk93,no.2,388(1967)].

[77] J. B. Birks. Scintillations from Organic Crystals: Specific Fluorescence and Relative Response to Different Radiations. Proc. Phys. Soc. A64, (1951) 874-877.

[78] C. N. Chou. The Nature of the Saturation Effect of Fluorescent Scintillators. Phys. Rev. 87, (1952) 904-905.

[79] M. Ester, H.-P. Kriegel, J. Sander, and X. Xu. A Density-based Algorithm for Discovering Clusters a Density-based Algorithm for Discovering Clusters in Large Spatial Databases with Noise. In Proceedings of the Second International Conference on Knowledge Discovery and Data Mining, KDD'96. AAAI Press, 1996 226-231. 
[80] M. Baird, J. Bian, M. Messier, E. Niner, D. Rocco, and K. Sachdev. Event Reconstruction Techniques in NOvA. J. Phys. Conf. Ser. 664, (2015) 072,035.

[81] L. A. F. Fernandes and M. M. Oliveira. Real-time Line Detection Through an Improved Hough Transform Voting Scheme. Pattern Recogn. 41, (2008) 299-314.

[82] M. Ohlsson. Extensions and explorations of the elastic arms algorithm. Comput. Phys. Commun. 77, (1993) 19-32.

[83] R. Krishnapuram and J. M. Keller. A Possibilistic Approach to Clustering. Trans. Fuz Sys. 1, (1993) 98-110.

[84] M.-S. Yang and K.-L. Wu. Unsupervised Possibilistic Clustering. Pattern Recogn. 39, (2006) $5-21$.

[85] E. D. Niner. Observation of Electron Neutrino Appearance in the NuMI Beam with the NOvA Experiment. Ph.D. thesis, Indiana U. 2015.

[86] R. Fruhwirth. Application of Kalman filtering to track and vertex fitting. Nucl. Instrum. Meth. A262, (1987) 444-450.

[87] C. Backhouse and A. Radovic. The Attenuation and Threshold Correction of the NOvA Detectors. NOvA Collaboration Internal document 13579 .

[88] K. A. Olive et al. Review of Particle Physics. Chin. Phys. C38, (2014) 090,001.

[89] L. A. et. al. Technical Note on the ND numu CC Inclusive Double-Differential Cross Section Measurement. NOvA internal Note-32688.

[90] M. Baird. A Side By Side Comparison of Slicer, Cosmic Slicer, and Slicer4D. NOvA internal Note-9195.

[91] N. Raddatz. KalmanTrack Technical Note. NOvA internal Note-13545.

[92] N. Raddatz. ReMId Technical Note. NOvA internal Note-11206.

[93] C. Backhouse. The CAFAna Framework technote. NOvA Collaboration Internal document 9222.

[94] L. Aliaga. NuMI Beam Prediction for the NOvA 2017 Analyses. NOvA internal Note-23441.

[95] L. Cremonesi. NuMI flux systematic uncertainties for the NOvA third analyses. NOvA internal Note-17608.

[96] C. Andreopoulos, C. Barry, S. Dytman, H. Gallagher, T. Golan, R. Hatcher, G. Perdue, and J. Yarba. The GENIE Neutrino Monte Carlo Generator: Physics and User Manual .

[97] S. Lin. Note for the Multi-universe approach for GENIE Systematics. NOvA internal Note21635 .

[98] K. Bays, A. Mislivec, G. Pawloski, and J. Wolcott. NOvA 2018 cross-section tune tech note. NOvA internal Note-27755. 
[99] S. Bending. ND Third Analysis Containment Study. NOvA internal Note-23336.

[100] C. Johnson. ReMId vs. MuonId Comparisons. NOvA internal Note-30930.

[101] S. M. Lein. $\nu_{\mu}$ Charged Current Energy Estimators. NOvA Collaboration Internal document 15211 .

[102] B. Behera. Tuning Energy Estimators: NumuCC XSec. NOvA internal Note-18756.

[103] B. Behera. Tuning Energy Estimators: NumuCC XSec. NOvA internal Note-19152.

[104] K. Sachdev. MRCC tech note. NOvA internal Note-9792.

[105] K. Sachdev. Numu CC Update: Summer 2017 Meeting. NOvA internal Note-20594.

[106] K. Sachdev. Numu CC Update: Data Efficiency Study. NOvA internal Note-21746.

[107] K. Sachdev. Numu CC: Data Efficiency Study Follow Up. NOvA internal Note-22283.

[108] S. Schmitt. Data Unfolding Methods in High Energy Physics. EPJ Web Conf. 137, (2017) 11,008 .

[109] A. Hocker and V. Kartvelishvili. SVD approach to data unfolding. Nucl. Instrum. Meth. A372, (1996) 469-481.

[110] A. N. Tikhonov. On the solution of improperly posed problems and the method of regularization. Sov. Math. 5, (1964) 1035.

[111] Nuclear Instruments and Methods in Physics Research A 476, (2002) 242-246.

[112] C. Backhouse. Derivation of flux. NOvA internal Note-15910.

[113] M. Strait. Muon Energy Scale; Mass Accounting. NOvA internal Note-20816.

[114] B. Behera. Intensity Effect: nu mu CC XSec. NOvA internal Note-19482.

[115] R. J. Tesarek. NOvA Near Detector Muon Catcher Steel Measurements. NOvA internal Note-16937.

[116] B. Zamorano. Misalignment systematic uncertainty for the first analysis. NOvA internal Note-13639.

[117] B. Behera. Technote on GEANT systematic study in Near Detector. NOvA internal Note15776 . 